\title{
ABSTRACT
}

MAHONEY, KELLY M. The Effect of Upstream Convection on Downstream Precipitation. (Under the direction of Gary M. Lackmann.)

Numerical weather prediction (NWP) models have demonstrated a weakness in the ability to accurately forecast precipitation amounts downstream of strong, organized convection in the Southeast US. This weakness has also been communicated by operational forecasters, as past events have exhibited reduced downstream precipitation amounts relative to the model quantitative precipitation forecast (QPF), particularly when the upstream convection (UC) feature moves rapidly eastward. Conventional forecaster wisdom evolving from such events thus advocates reducing downstream QPF in the presence of UC.

The purposes of this study are (i) to identify the physical processes by which downstream precipitation may be reduced or enhanced in the presence of UC; (ii) distinguish UC cases in which downstream QPF should be reduced from those in which it should be enhanced; (iii) understand why operational models are challenged to produce an accurate downstream forecast in these situations; (iv) identify synoptic settings associated with different types of UC events; (v) find ways in which human forecasters may anticipate and improve upon erroneous model forecasts; and (vi) investigate optimal model configurations for the representation of UC and downstream QPF.

A brief climatology of National Centers for Environmental Prediction (NCEP) Eta model QPF error revealed that the UC problem is observed to occur in three relatively distinct synoptic settings, in which different QPF biases tended to occur. The three scenarios are based on the orientation and movement of the UC. Scenario 1 (S1) is characterized by UC that is oriented parallel to mean flow, and the organized convective 
system propagates quickly eastward relative to the primary synoptic system in a direction perpendicular to flow. These cases were generally characterized by a positive model QPF bias. Scenario 2 (S2) features UC that is oriented parallel to mean flow as in S1, but that propagates slowly (or not at all) with respect to the primary synoptic system. It was hypothesized that in these cases a diabatically-enhanced low-level jet (LLJ) would act to enhance moisture transport ahead of the synoptic system, and these cases were therefore hypothesized to yield increased downstream precipitation amounts, albeit with smaller QPF errors. Scenario 3 (S3) is characterized by UC oriented perpendicular to flow, usually aligning with a coastal and/or warm frontal features. S3 cases were found to often transition into an S1 or S2 event. They were consequently treated as precursors to S1 or S2 cases and are not investigated in great detail here.

Several potential physical mechanisms of downstream precipitation alteration were examined for each scenario. These physical mechanisms include (i) moisture consumption; (ii) stabilization of the downstream environment; (iii) alteration of lowertropospheric moisture transport through interruption (S1) or enhancement (S2) of the LLJ; and (iv) alteration of synoptic dynamics.

Two main case studies were undertaken to investigate S1 and S2. For the S1 case it was found that a high-resolution (4-km grid-spacing) convection-resolving forecast using the Weather Research and Forecast (WRF) model vastly improved the downstream QPF relative to the large overprediction of precipitation produced by the operational Eta forecast. Comparisons were made between the successful WRF run, analyses, and the erroneous operational Eta forecast. Errors in the low-level wind and horizontal moisture flux fields from the operational Eta forecast revealed that the delayed speed of the model- 
forecasted UC did not permit an accurate representation of low-level moisture transport (physical mechanism (iii)), causing the model to allow too much moisture to infiltrate the downstream area, and likely contributing to the model overprediction of precipitation. The S1 case showed that despite excellent operational Eta forecasts of the placement and intensity of the synoptic system, errors in model-forecasted convective motion resulted in large operational QPF errors downstream.

The S2 case study revealed that UC does not always lead to a decrease in downstream precipitation. Potential vorticity (PV) diagnostics were used to demonstrate that the diabatic influence of the UC acted to enhance the low-level jet (LLJ) and increase moisture transport (physical mechanism (iii)). A quasi-geostrophic PV inversion showed that the diabatic cyclonic QGPV anomaly associated with the UC feature contributed significantly to the southerly LLJ preceding the convective line. S2 proved to be an important counter-example to the notion that UC generally reduces downstream precipitation, as this case study demonstrates a physical process associated with the UC that instead acts to enhance downstream precipitation.

The findings of this study may be helpful to forecasters in anticipating future UC events, as well as to numerical modelers facing decisions regarding what type of model run will yield the best results for future UC events. The results are further analyzed in the context of the ability of cumulus parameterization (CP) schemes to accurately represent convective movement, and the implications of such a shortcoming for UC forecasts. Future work in this area is suggested. 


\title{
THE EFFECTS OF UPSTREAM CONVECTION ON DOWNSTREAM
} PRECIPITATION

\author{
by \\ KELLY MARIE MAHONEY
}

A thesis submitted to the Graduate Faculty of

North Carolina State University

in partial fulfillment of the

requirements for the Degree of

Master of Science

MARINE, EARTH, AND ATMOSPHERIC SCIENCES

Raleigh, NC

2005

APPROVED BY:

Gary M. Lackmann

(Chair of Advisory Committee)

Allen J. Riordan

Sethu Raman

(Substitute for Lian Xie) 


\section{PERSONAL BIOGRAPHY}

Kelly Mahoney was born in Baltimore, Maryland and grew up in Ellicott City, Maryland. She first became interested in the weather during a run-in with Hurricane Bertha on a family beach vacation, and shortly thereafter began learning all that she could about meteorology. She came to NC State in the fall of 1999 with the help of a Park Scholarship and completed her Bachelor's degree in 2003, graduating summa cum laude with a major in Meteorology and a minor in Spanish. During her time as an undergraduate, Kelly was an active participant in groups and activities such as Hope for the Homeless and WKNC radio, as well as both the NC State Student and Central North Carolina chapters of the American Meteorological Society. She was also fortunate enough to be able to fill her college summers storm-chasing, interning at WBAL-TV and WRAL-TV, and doing undergraduate research.

Kelly happily accepted an offer to stay at NC State for graduate school in 2003 and received an AMS graduate fellowship for her first year of study. She was fortunate enough to be able to do her Master's research under the CSTAR project, collaborating with National Weather Service forecasters to examine the effects of upstream convection on downstream precipitation forecasts. Upon completion of her Master of Science degree, Kelly plans to continue her graduate studies by remaining at NC State to pursue a Doctor of Philosophy degree in Atmospheric Science. 


\section{ACKNOWLEDGEMENTS}

Support for this research was received from the NOAA Collaborative Science,

Technology, and Applied Research (CSTAR) program (Grant \# NA03NWS4680007) awarded to North Carolina State University, as well as an American Meteorological Society (AMS) graduate fellowship award.

The National Centers for Environmental Prediction (NCEP) provided much of the meteorological data used in this study, which was delivered to NC State through the Unidata program. Thank you to Chris Bailey of the Hydrometeorological Prediction Center (HPC) and Matt Pyle of NCEP for supplying forecast and model data. The National Center for Atmospheric Research (NCAR) is also acknowledged for the availability of the WRF and MM5 models, as is Ryan Torn of University of Washington for the original WRF to GEMPAK converter. Analyses provided by the North American Regional Reanalysis (NARR) project and the National Precipitation Verification Unit (NPVU) are also very much appreciated.

I would like to express my deep gratitude to my committee members, Drs. Gary Lackmann, Allen Riordan, and Lian Xie, as well as to Dr. Sethu Raman for volunteering to be a committee substitute. Their advice and suggestions on developing and improving my research are appreciated tremendously. In particular I would like to thank my advisor, Dr. Lackmann for his infinite patience and constant willingness to help, teach, and challenge me. His support, encouragement, and friendship made this experience a rich and rewarding one from which I learned so much.

Second, I would like to thank the wonderful people from the National Weather Service and the CSTAR program in general. This unique program allowed me to work on 
research in which I was very much interested, and allowed me to learn from those whose experience both motivated this research and who were able to offer help and suggestions along the way. In particular I would like to thank Kermit Keeter, Rod Gonski, Gail Hartfield, Jonathan Blaes, Ron Humble, Jeff Waldstreicher, Steve Zubrick, Steve Keighton, and Larry Lee for their interest, assistance, and insight.

I have also been very fortunate to work with a wonderful group of people over the past few years. Thanks to everyone in the Forecasting Lab (past members and present) as well as to the whole RIII crew for all of the help, support, encouragement and healthy diversion. And of course, my deepest thanks go out to Dr. Michael Brennan for more help than any one person should ever have to give, and without whom I would still probably be staring blankly at the first MM5 code, sitting in a puddle of my own tears! Your friendship and generosity has been -- and will remain -- very much appreciated!

Finally, I would like to express my most sincere gratitude to my family and friends. First, thank you to my parents, Bill and Gina, and to my brother Billy for unconditional love, support, laughs, and reality-checks. Thanks especially to my parents for instilling in me from the beginning a deep appreciation for learning and education. Thank you to my fiancé Scott Jackson for his limitless and unwavering support and encouragement. Thank you especially for standing by me when I need it, yet still always fostering and encouraging my confidence and independence. The valuable friendships that I've been able to count on over the past few years has also been a huge source of strength for me as well, so thank you to everyone who I've been able to call a friend, but especially to Carrie, Tristan, Kirsten, Angela, Bryan, Kevin, Noelle, Courtney, Becky, and Sara - I couldn't have done it without your love and support over the years! 


\section{TABLE OF CONTENTS}

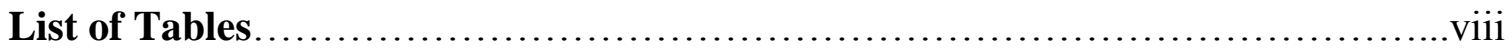

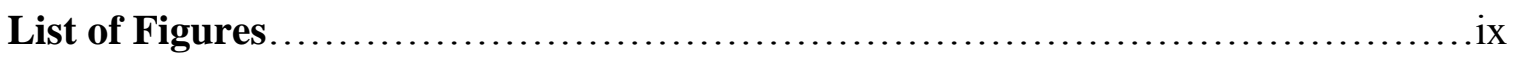

\section{Introduction}

1.1 Motivation...............................................................

1.2 Hypotheses - physical processes and primary scenarios.................... 3

1.2.1 Mechanisms of downstream precipitation alteration................3

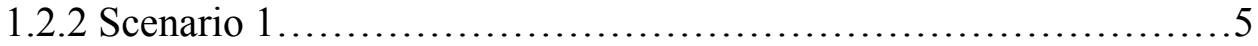

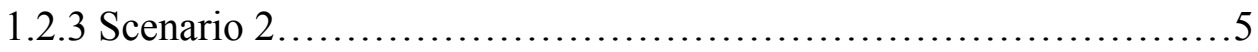

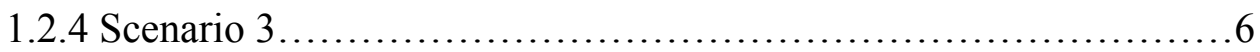

1.3 Background Research..............................................6

1.3.1 Organized Convection......................................

1.3.2 Mesoscale Convective Systems (MCSs)......................

1.3.2.1. MCS downdraft dynamics ..........................8

1.3.2.2 MCS movement and propagation......................10

1.3.3 Representation of convection in numerical models......................14

1.3.4 Role of a diabatically enhanced LLJ...............................17

\section{Methodology}

2.1 Eta model QPF error climatology and case selection.......................34

2.2 Numerical simulations and forecasts....................................

2.2.1 Mesoscale models.............................................37

2.2.1.1 PSU-NCAR mesoscale model (MM5)....................37

2.2.1.2 Weather Research and Forecast (WRF) model.............38

2.2.2 Mesoscale model runs: WRF S1 case study.......................39

2.2.3 Mesoscale model runs: WRF S2 case study....................40

2.3 PV methodology ..............................................41

2.3.1 Ertel PV budget..........................................42

2.3.2 Quasigeostrophic potential vorticity inversion..................43

3. S1 Case Study: 31 Dec 2002 - 01 Jan 2003

3.1 Introduction/event summary 


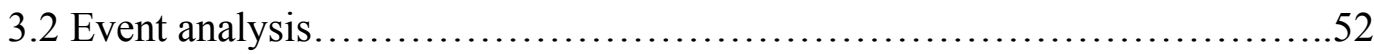

3.2.1 Observational analysis.........................................

3.2.1.1 Synoptic scale analysis ...............................52

3.2.1.2 Mesoscale analysis....................................53

3.2.2 Analysis of operational forecast.................................. 54

3.2.2.1 Assessment of Eta QPF................................55

3.2.2.2 Eta synoptic scale forecast analysis......................56

3.2.2.3 Eta mesoscale forecast analysis.......................57

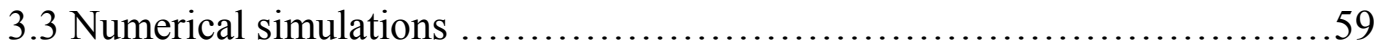

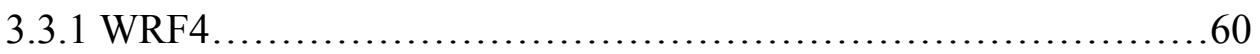

3.3.1.1 Assessment of WRF4 QPF..........................60

3.3.1.2 WRF4 mesoscale forecast analysis......................61

3.3.1.3 Physical mechanisms of downstream precipitation

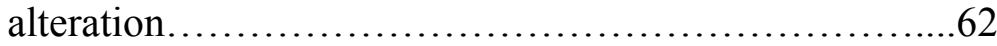

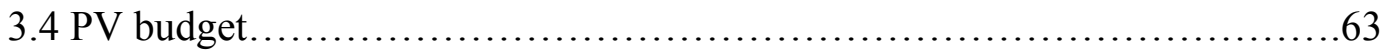

3.4.1 WRF20 forecast evaluation......................................64

3.4.2 PV budget results........................................... 65

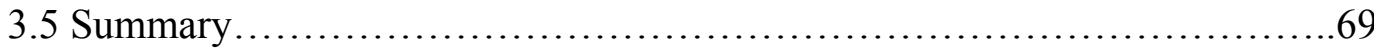

4. Scenario 2: 13 - 14 January 2005

4.1 Introduction/event summary ........................................ 105

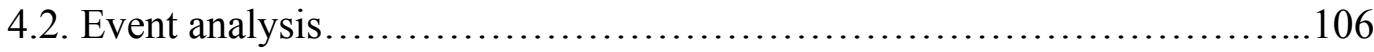

4.2.1 Observational analysis ....................................... 106

4.2.1.1 Synoptic scale analysis............................106

4.2.1.2 Mesoscale/Convective scale/Storm scale analysis.......107

4.2.2 Analysis of operational forecast................................109

4.2.2.1 Assessment of Eta QPF...............................109

4.2.2.2 Eta synoptic scale forecast fields......................110

4.2.2.3 Eta mesoscale forecast fields.........................112

4.2.2.4 Other forecast considerations ..........................113

4.3 Numerical simulations ................................................ 113 
4.3.1 WRF control run.........................................114

4.3.2 Assessment of WRF control run................................115

4.3.2.1 Assessment of WRF QPF.............................115

4.3.2.2 WRF synoptic forecast features........................116

4.3.2.3 WRF mesoscale forecast features.....................117

4.4 PV investigation ................................................... 119

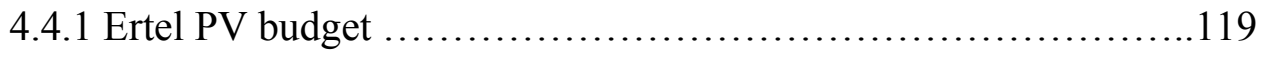

4.4.2 QGPV inversion............................................ 121

4.4.2.1 The use of QGPV ..................................121

4.4.2.2 Results of the QGPV inversion.......................122

\section{Discussion, Applications, and Conclusions}

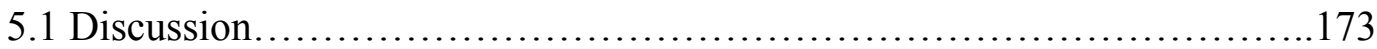

5.1.1 Scenario 3 and the continuum of UC environments ..............173

5.1.2 PV budget comparisons .....................................174

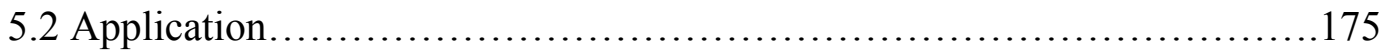

5.2.1 The nature and importance of UC system motion ................175

5.2.2 Forecasting considerations....................................177

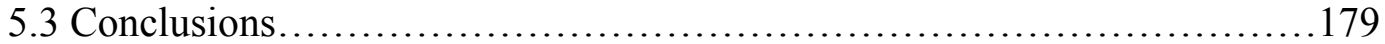

5.3.1. Objectives................................................ 179

5.3.2 Hypotheses.............................................. 180

5.3.3 Case Studies and model experiments.............................182

5.3.3.1 Scenario 1: 31 Dec 2002 - 1 Jan 2003 ..................183

5.3.3.2 Scenario 2: 13 -14 Jan 2005......................... 184

5.3.4 Forecasting implications ................................... 185

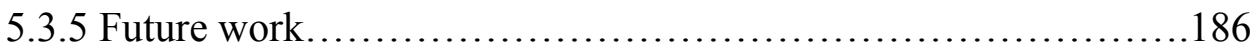

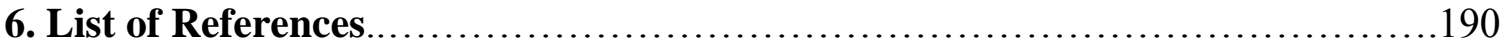

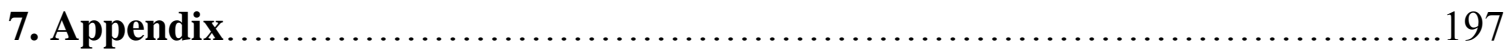




\section{LIST OF TABLES}

Table 1.1. Richardson number $\mathrm{R}$ as calculated for a series of documented storms. Model results are summarized at the top of the figure. S1, S2, .., S9 represent supercell storms; M1, M2, .., M5 represent multicell storms; TR1, TR2,..., TR4 represent tropical cases (from Weisman and Klemp 1986 after Weisman and Klemp 1982)........................... 32

Table 1.2. Storm type as a function of vertical shear and CAPE (from Bluestein 1993, Table 3.2)................................................33

Table 2.1 Sample selection of the Eta QPF error climatology....................51

Table 5.1. Environmental characteristics common to S1 and S2 events.............189 


\section{LIST OF FIGURES}

Figure 1.1. Area Forecast Discussions from National Weather Service forecast offices referencing the challenge that UC poses to the downstream

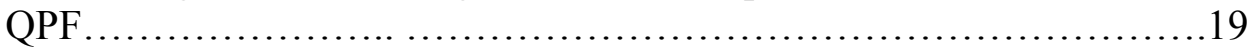

Figure 1.2. Schematic of physical mechanism (i), consumption....................20

Figure 1.3. Schematic of physical mechanism (ii), stabilization.....................21

Figure 1.4. (a) Schematic of physical mechanism, (iii) interruption of lowertropospheric moisture transport (S1); (b) as in (a), except for S2, showing the enhancement of lower-tropospheric moisture transport.......................22

Figure 1.5. Schematic of physical mechanism (iv) alteration of synoptic dynamics...23

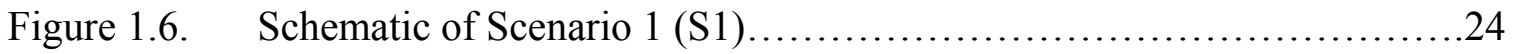

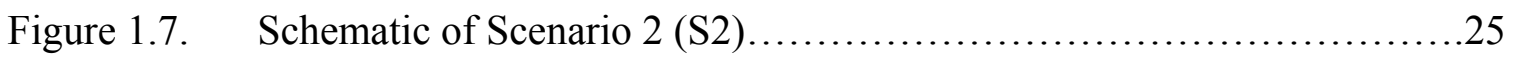

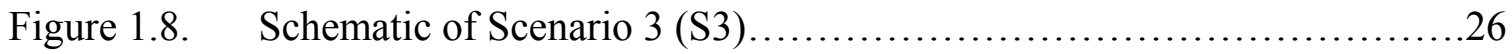

Figure 1.9. (a) Idealization of a horizontal map of radar reflectivity (b) divided into convective and stratiform regions (from Houze 1997)...................27

Figure 1.10. Idealization of a mesoscale convective system with leading-line/trailing stratiform structure, showing branches of flow and position of surface mesohighs and mesolows (from Houze 2004 after Zipser 1977)..........28

Figure 1.11. Schematic of a mature squall system. Surface pressure is contoured, vectors represent surface wind, and significant regions of precipitation are stippled (from Haertel and Johnson 2000 after Johnson and Hamilton 1988).

Figure 1.12. Idealized cross section through slab convective overturning. Flow vectors are system relative, scalloped lines indicate cloud boundaries, solid lines are $\theta_{\mathrm{e}}$ contours every $4 \mathrm{~K}$ (thin dashed line is an intermediate contour, and bold dashed line marks axis of highest values), bold solid line indicates outflow boundary or frontal zone, light shading highlights middle level layer of low $-\theta_{\mathrm{e}}$ air, and dark shading depicts the moist absolutely unstable layer (MAUL) (from Houze 2004 after Bryan and Fritsch 2000)..........30

Figure 1.13. (a) Conceptual model of MCS movement $\left(\mathrm{V}_{\mathrm{MBE}}\right)$ as the vector sum of the mean flow in the cloud layer $\left(V_{\mathrm{CL}}\right)$ and the propagation component $\left(V_{\mathrm{PROP}}\right)$. The magnitude and direction of $V_{\mathrm{PROP}}$ are assumed to be equal 
and opposite to those of the low-level jet $\left(V_{\mathrm{LLJ}}\right)$. The angles $f$ and $\mathrm{w}$ are used to calculate $V_{\mathrm{MBE}}$ given observed values of $V_{\mathrm{CL}}$ and $V_{\mathrm{LLJ}}$. Dashed lines (labeled THKNS) indicate a typical relationship of the 850-300-mb thickness pattern to the environmental flow and MBE movement during MCC events (from Corfidi 1996); (b) Schematic of the original vector technique, with MCS core motion (thick dotted arrow) expressed as the vector sum of 1) advection of cells by the mean cloud-layer wind (arrow pointing to upper right) and 2) cell propagation directed into the low-level jet (arrow pointing to bottom of page). MCS centroid is depicted by the cross symbol (from Corfidi 2003 after Corfidi et al. 1996).................31

Figure 2.1 Area over which Eta forecasts and NARR precipitation analyses were averaged for the Eta QPF error climatology. Color shading denotes the gridcells contained by the $200-\mathrm{km}$ radius from RDU....................46

Figure 2.2 Model domain used in S1 MM5 simulation...........................47

Figure 2.3 Model domain used in S1 WRF4 forecast...........................48

Figure 2.4 Model domain used in S1 WRF20 forecast............................49

Figure 2.5 Model domain used in S2 WRF forecast............................50

Figure 3.1. Severe weather reports as reported by the Storm Prediction Center for 31

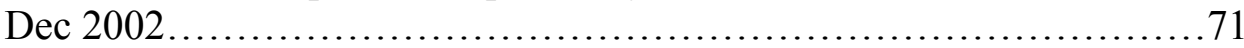

Figure 3.2. Radar mosaic imagery valid at: (a) 12 UTC 31 Dec; (b) 15 UTC 31 Dec (due to missing data, image obtained from NCDC online NEXRAD radar archive); (c) 18 UTC 31 Dec; (d) 21 UTC 31 Dec; (e) 00 UTC 1 Jan; (f) 03 UTC 1 Jan; (g) 06 UTC 1 Jan; (h) 09 UTC 1 Jan; (i) 12 UTC 1 Jan................................................................. 72

Figure 3.3. (a) 24-h total precipitation forecast ending 00 UTC 02 Jan by operational Eta model (mm, shaded as in colorbar in lower right corner; (b) 24-h NARR analysis precipitation totals ending 00 UTC 02 Jan ( $\mathrm{mm}$, shaded as in colorbar in lower right corner)........................................73

Figure 3.4. Eta Data Assimilation System (EDAS) analysis synoptic summary for 12 UTC 31 Dec 2002: (a) 250-hPa Geopotential height (solid contours, interval 12 dam) and isotachs (kt, shaded as in legend at right of panel); (b) 500-hPa Geopotential height (solid, interval 6 dam) and vorticity $\left(\times 10^{-5} \mathrm{~s}^{-1}\right.$, shaded as in legend at right of panel); (c) 850 -hPa potential temperature (dashed contours, interval $2 \mathrm{~K}$ ), $850-\mathrm{hPa}$ Geopotential height (solid, interval $12 \mathrm{dam}$ ), and 850-hPa mixing ratio (shaded, interval 2 
$\mathrm{g} / \mathrm{kg}$, values below $6 \mathrm{~g} / \mathrm{kg}$ omitted); (d) sea level pressure (solid contours, interval $2 \mathrm{hPa}$ ) and 2 -m temperature (dashed contours, interval $2^{\circ} \mathrm{C}$ )......74

Figure 3.5. As in Fig. 3.4, except for 00 UTC 01 Jan 2003........................75

Figure 3.6. As in Fig. 3.4, except for 12 UTC 01 Jan 2003.........................76

Figure 3.7. Eta Data Assimilation System (EDAS) analysis mesoscale summary for 12 UTC 31 Dec 2002: (a) 500-950-hPa equivalent potential temperature difference (solid contours, interval $2 \mathrm{~K}$, positive values omitted) and 700950-hPa wind shear $(10 \mathrm{kt} / \mathrm{m}$, shaded as in legend at right of panel); (b) 900-800 layer-averaged PV (PVU (1 PVU $\left.=10^{-6} \mathrm{~K} \mathrm{~m}^{2} \mathrm{~kg}^{-1} \mathrm{~s}^{-1}\right)$, shaded as in legend at right of panel) and $850-\mathrm{hPa}$ winds (kt, barbs); (c) $850-\mathrm{hPa}$ Geopotential height (solid, interval 4 dam), isotachs (kt, shaded as in legend at right of panel), and 850-hPa winds (kt, barbs); (d) 850-hPa moisture flux $\left(\mathrm{g} \mathrm{kg}^{-1} \mathrm{~m} \mathrm{~s}^{-1}\right.$, shaded as in legend at right of panel) and moisture flux vectors (arrows) .......................................77

Figure 3.8. (a) NEXRAD radar reflectivity at 12 UTC 31 Dec; (b) observed FFC sounding at 12 UTC 31 Dec; (c) observed JAX sounding at 12 UTC 31

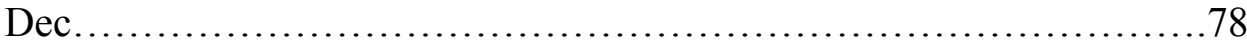

Figure 3.9. As in Fig. 3.7, except for 00 UTC 01 Jan 2003.........................79

Figure 3.10. As in Fig. 3.8, except for 12 UTC 01 Jan 2003........................80

Figure 3.11. (Row A) (from left to right): NARR 6-h precipitation analyses ending at 12 UTC 31 Dec, 18 UTC 31 Dec, 00 UTC 01 Jan, 06 UTC 01 Jan, 12 UTC 01 Jan, respectively; (Row B) as in (Row A) except for operational Eta forecast; (Row C) as in (Row A) except for MM5 KF model simulation; (Row D) as in (Row A) except for WRF20 forecast; (Row E) as in (Row A) except for WRF4 forecast............................... 81

Figure 3.12. Difference field between Eta 211 24-h QPF and NARR analysis 24-h total from 24-h period ending 00 UTC 02 Jan (mm, shaded as in legend at left

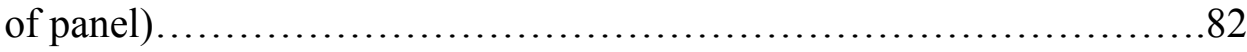

Figure 3.13. (a) 6-h total precipitation forecast by operational Eta model ending 12 UTC 1 Jan (mm, shaded as in legend at right of panel; (b) 6-h NARR analysis precipitation totals ending 12 UTC $1 \mathrm{Jan}(\mathrm{mm}$, shaded as in

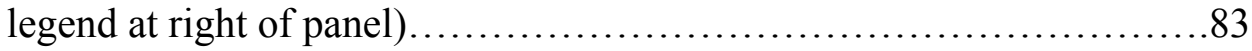

Figure 3.14. Operational Eta 12-h forecast valid 12 UTC 31 Dec 2002: (a) 250-hPa Geopotential height (solid contours, interval 12 dam) and isotachs (kt, shaded as in legend at right of panel); (b) 500-hPa Geopotential height 
(solid, interval 6 dam) and vorticity $\left(\times 10^{-5} \mathrm{~s}^{-1}\right.$, shaded as in legend at right of panel); (c) 850-hPa potential temperature (dashed contours, interval $2 \mathrm{~K}$ ), 850 -hPa Geopotential height (solid, interval 12 dam), and $850-\mathrm{hPa}$ mixing ratio (shaded, interval $2 \mathrm{~g} / \mathrm{kg}$, values below $6 \mathrm{~g} / \mathrm{kg}$ omitted); (d) sea level pressure (solid contours, interval $2 \mathrm{hPa}$ ), 2-m temperature (dashed contours, interval $2^{\circ} \mathrm{C}$ ), and 6-h accumulated precipitation ( $\mathrm{mm}$, shaded as in legend at left of panel) ending 12 UTC 31

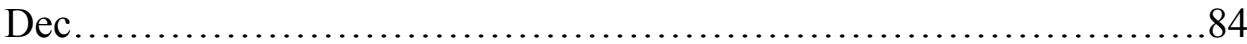

Figure 3.15. As in Fig. 3.14 except for 24-h forecast valid 00 UTC 1 Jan.............85

Figure 3.16. As in Fig. 3.14 except for 36-h forecast valid 12 UTC 1 Jan...............86

Figure 3.17. Operational Eta 12-h forecast valid 12 UTC 31 Dec 2002: (a) 500-950$\mathrm{hPa}$ equivalent potential temperature difference (solid contours, interval 2 $\mathrm{K}$, positive values omitted) and $700-950-\mathrm{hPa}$ wind shear $(10 \mathrm{kt} / \mathrm{m}$, shaded as in legend at right of panel); (b) 900-800 layer-averaged PV (PVU, shaded as in legend at right of panel) and $850-\mathrm{hPa}$ winds (kt, barbs); (c) 850-hPa Geopotential height (solid contours, interval 4 dam), isotachs (kt, shaded as in legend at right of panel), and 850-hPa winds (kt, barbs); (d) 850-hPa moisture flux ( $\mathrm{g} \mathrm{kg}^{-1} \mathrm{~m} \mathrm{~s}^{-1}$, shaded as in legend at right of panel) and moisture flux vectors (arrows) ................................. 87

Figure 3.18. As in Fig. 3.17 except for 24-h forecast valid 00 UTC 1 Jan.............88

Figure 3.19. As in Fig. 3.17 except for 36-h forecast valid 12 UTC 1 Jan..............89

Figure 3.20. (a) 24-h Eta model forecast total QPF ending 00 UTC 02 Jan (mm, shaded as in legend at left of panel); (b) as in (a) except NARR analysis; (c) as in (a) except for WRF4 forecast. .90

Figure 3.21. WRF4 12-h forecast valid 12 UTC 31 Dec 2002: (a) 500-950-hPa equivalent potential temperature difference (solid contours, interval $2 \mathrm{~K}$, positive values omitted) and 700-950-hPa wind shear (10 kt/m, shaded as in legend at right of panel); (b) 900-800 layer-averaged PV (PVU, shaded as in legend at right of panel) and $850-\mathrm{hPa}$ winds (kt, barbs); (c) $850-\mathrm{hPa}$ Geopotential height (solid, interval 4 dam), isotachs (kt, shaded as in legend at right of panel), and 850-hPa winds (kt, barbs); (d) 850-hPa moisture flux $\left(\mathrm{g} \mathrm{kg}^{-1} \mathrm{~m} \mathrm{~s}^{-1}\right.$, shaded as in legend at right of panel) and moisture flux vectors (arrows) ....................................91

Figure 3.22. As in Fig. 3.21 except for 24-h forecast valid 00 UTC 1 Jan............92

Figure 3.23. (a) 850-hPa Geopotential height (solid contours, interval 4 dam), 850-hPa winds (kt, barbs), and $850-\mathrm{hPa}$ isotachs (kt, shaded as in legend at right of 
panel) for Eta analysis valid 00 UTC 1 Jan; (b) 850-hPa moisture flux magnitude $\left(\mathrm{g} \mathrm{kg}^{-1} \mathrm{~m} \mathrm{~s}^{-1}\right.$, shaded as in legend at right of panel) and moisture flux vectors (arrows) for Eta analysis valid 00 UTC 1 Jan; (c) as in (a), except for 12-h Eta forecast; (d) as in (b), except for 12-h Eta forecast, (e) as in (a), except for 12-h WRF4 forecast, (f) as in (b), except for 12-h WRF4 forecast.

Figure 3.24. As in Fig. 3.21 except for 36-h forecast valid 12 UTC 1 Jan.............94

Figure 3.25. (a) 800-900-hPa PV (PVU, shaded as in legend at right of panel) and 850$\mathrm{hPa}$ parameterized latent heating (solid contours, $\mathrm{K}$ day $^{-1}$ ) for $12-\mathrm{h}$ forecast valid 12 UTC $31 \mathrm{Dec}$; (b) as in (a) except for 24-h forecast valid 00 UTC 01 Jan; (c) as in (a) except for 36-h forecast valid 12 UTC 01 Jan. Solid line A-A' in (a) denotes cross-section shown in Fig. 3.28, solid line B-B' in (b) denotes cross-section shown in Fig. 3.29, solid line C-C' in (b) denotes cross-section shown in Fig. 3.32, and solid line D-D' in (c) denotes cross-section shown in Fig. 3.34c .95

Figure 3.26. PV budget summary for 12 UTC 31 Dec: (a) 800-900-hPa PV (PVU, shaded as in legend at right of panel) and $850-\mathrm{hPa}$ horizontal advective PV tendency (contour interval $1 \mathrm{PVU}^{\mathrm{Pay}}{ }^{-1}$, red (blue) contours correspond to positive (negative) values); (b) as in (a) except for the vertical advective PV tendency; (c) as in (a) except for nonadvective PV tendency; (d) as in (a) except for total PV tendency...............................96

Figure 3.27. As in Fig. 3.26 except for 00 UTC 01 Jan..........................97

Figure 3.28. Cross section taken along line A-A' in Fig. 3.25a valid at 12 UTC 31 Dec showing parameterized latent heating $\left(\mathrm{K} \mathrm{day}^{-1}\right.$, shaded as in legend at bottom of panel), PV (solid contours, PVU), and absolute vorticity vectors

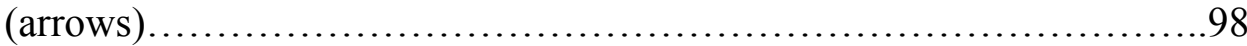

Figure 3.29. As in Fig. 3.28 except cross-section taken along line B-B' in Fig. 3.25b and valid at 00 UTC 01 Jan........................................99

Figure 3.30. Cross section taken along line A-A' in Fig. 3.25a valid at 12 UTC 31 Dec showing PV (solid contours, PVU), absolute vorticity vectors (arrows), and section-normal wind speed (kt, shaded as in legend at bottom of

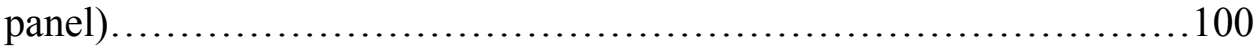

Figure 3.31. As in Fig. 3.30 except cross-section taken along line B-B' in Fig. 3.25b

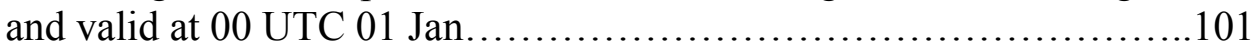

Figure 3.32. As in Fig. 3.31 except cross-section taken along line C-C' in Fig. $3.25 b$. 102 
Figure 3.33. (a) Cross section taken along line A-A' in Fig. 3.25a valid at 12 UTC 31 Dec showing PV (PVU, shaded as in legend at bottom of panel), nonadvective PV tendency (contour interval $1 \mathrm{PVU}^{\mathrm{P}} \mathrm{day}^{-1}$, solid (dashed) lines denote positive (negative) values), and non-advective PV flux vectors (arrows); (b) as in (a) except cross-section taken along line B-B' in Fig. $3.25 \mathrm{~b}$ and valid at 00 UTC $01 \mathrm{Jan}$; (c) as in (a) except cross-section taken along line D-D' in Fig. 3.25c and valid at 12 UTC 01 Jan................103

Figure 3.34. As in Fig. 3.33 except for advective PV tendency.......................104

Figure 4.1. Surface analysis and surface observations from Hydrometeorological Prediction Center (HPC), valid 0415 UTC 14 Jan 2005, using conventional symbols and notation..................................124

Figure 4.2. Radar mosaic imagery valid at: (a) 00 UTC 13 Jan; (b) 06 UTC 13 Jan; (c) 12 UTC 13 Jan; (d) 18 UTC 13 Jan; (e) 00 UTC 14 Jan; (f) 06 UTC 14 Jan; (g) 12 UTC 14 Jan; (h) 18 UTC 14 Jan; (i) 00 UTC 15 Jan.........125

Figure 4.3. Severe weather reports as reported by the Storm Prediction Center for 13

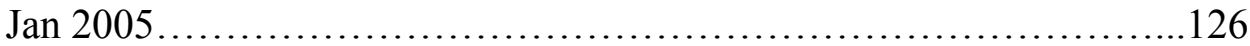

Figure 4.4. 24-h observed precipitation totals (numbers) and the operational Eta (initialized 00 UTC 13 Jan) 24-h QPF (inches, solid contours), valid 12 UTC 14 Jan 127

Figure 4.5. Eta Data Assimilation System (EDAS) analysis synoptic summary for 00 UTC 13 Jan 2005: (a) 250-hPa Geopotential height (solid contours, interval $12 \mathrm{dam}$ ) and isotachs (kt, shaded as in legend at right of panel); (b) $500-\mathrm{hPa}$ Geopotential height (solid, interval 6 dam) and vorticity $\left(\times 10^{-5} \mathrm{~s}^{-1}\right.$, shaded as in legend at right of panel); (c) $850-\mathrm{hPa}$ potential temperature (dashed contours, interval $2 \mathrm{~K}$ ), 850-hPa Geopotential height (solid, interval $6 \mathrm{dam}$ ), and 850-hPa mixing ratio (shaded, interval $2 \mathrm{~g} / \mathrm{kg}$, values below $6 \mathrm{~g} / \mathrm{kg}$ omitted); (d) sea level pressure (solid contours, interval $2 \mathrm{hPa}$ ), 2-m temperature (dashed contours, interval $2^{\circ} \mathrm{C}$ ), and mosaic radar imagery $(\mathrm{dBz}$, shaded as in legend at left of panel)........128

Figure 4.6. As in Fig. 4.5 except for 12 UTC 13 Jan............................129

Figure 4.7. As in Fig. 4.5 except for 00 UTC 14 Jan.......................... 130

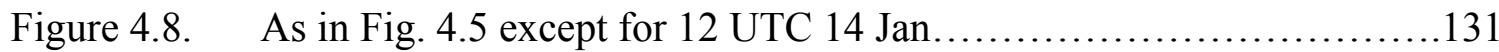

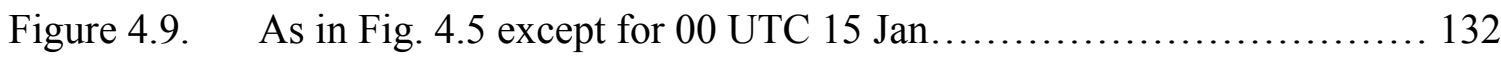


Figure 4.10. EDAS 250-hPa Geopotential height (solid contours, interval 12 dam), isotachs (kt, shaded as in legend at right of panel), and divergence (dashed contours, interval $\left.2 \times 10^{-5} \mathrm{~s}^{-1}\right)$ valid 12 UTC 14 Jan....................133

Figure 4.11. Eta Data Assimilation System (EDAS) analysis mesoscale summary for 00 UTC 13 Jan 2005: (a) 500-950-hPa equivalent potential temperature difference (solid contours, interval $2 \mathrm{~K}$, positive values omitted) and 700950-hPa wind shear (10 kt/m, shaded as in legend at right of panel); (b) 900-800 layer-averaged PV (PVU, shaded as in legend at right of panel) and 850-hPa winds (kt, barbs); (c) 850-hPa Geopotential height (solid, interval 4 dam), isotachs (kt, shaded as in legend at right of panel), and 850-hPa winds (kt, barbs); (d) 850-hPa moisture flux $\left(\mathrm{g} \mathrm{kg}^{-1} \mathrm{~m} \mathrm{~s}^{-1}\right.$, shaded as in legend at right of panel) and moisture flux vectors (arrows)........134

Figure 4.12. As in Fig. 4.11 except for 12 UTC 13 Jan.............................135

Figure 4.13. As in Fig. 4.11 except for 00 UTC 14 Jan..............................136

Figure 4.14. (a) NEXRAD radar reflectivity at 00 UTC 14 Jan; (b) observed TLH sounding at 00 UTC 14 Jan; (c) observed CHS sounding at 00 UTC 14 Jan................................................................ 137

Figure 4.15. As in Fig. 4.11 except for 12 UTC 14 Jan.............................138

Figure 4.16. As in Fig. 4.11 except for 00 UTC 15 Jan.............................139

Figure 4.17. 6-h precipitation estimates from the National Precipitation Verification Unit (NPVU) online archive valid for 6-h period: (a) 00 UTC 13 Jan; (b) 06 UTC 13 Jan; (c) 12 UTC 13 Jan; (d) 18 UTC 13 Jan; (e) 00 UTC 14 Jan; (f) 06 UTC 14 Jan; (g) 12 UTC 14 Jan; (h) 18 UTC 14 Jan; (i) 00 UTC 15 Jan 140

Figure 4.18. 6-h QPFs from operational Eta model initialized 00 UTC 13 Jan, valid for 6-h period ending: (a) (omitted; no QPF at F000); (b) 06 UTC 13 Jan; (c) 12 UTC 13 Jan; (d) 18 UTC 13 Jan; (e) 00 UTC 14 Jan; (f) 06 UTC 14 Jan; (g) 12 UTC 14 Jan; (h) 18 UTC 14 Jan; (i) 00 UTC 15 Jan..........141

Figure 4.19. Operational Eta 12-h forecast valid 12 UTC 13 Jan 2005: (a) 250-hPa Geopotential height (solid contours, interval 12 dam) and isotachs (kt, shaded as in legend at right of panel); (b) 500-hPa Geopotential height (solid, interval 6 dam) and vorticity $\left(\times 10^{-5} \mathrm{~s}^{-1}\right.$, shaded as in legend at right of panel); (c) 850-hPa potential temperature (dashed contours, interval $2 \mathrm{~K}$ ), $850-\mathrm{hPa}$ Geopotential height (solid, interval 6 dam), and $850-\mathrm{hPa}$ mixing ratio (shaded, interval $2 \mathrm{~g} / \mathrm{kg}$, values below $6 \mathrm{~g} / \mathrm{kg}$ omitted); (d) sea level pressure (solid contours, interval $2 \mathrm{hPa}$ ), 2-m 
temperature (dashed contours, interval $2^{\circ} \mathrm{C}$ ), and 6-h accumulated precipitation (mm, shaded as in legend at left of panel) ending 12 UTC 13 Jan.

Figure 4.20. As in Fig. 4.19 except for 00 UTC 14 Jan............................143

Figure 4.21. As in Fig. 4.19 except for 12 UTC 14 Jan............................144

Figure 4.22. As in Fig. 4.19 except for 00 UTC 15 Jan.............................145

Figure 4.23. Operational Eta 12-h forecast valid 12 UTC 13 Jan 2005: (a) 500-950-hPa equivalent potential temperature difference (solid contours, interval $2 \mathrm{~K}$, positive values omitted) and 700-950-hPa wind shear (10 kt/m, shaded as in legend at right of panel); (b) 900-800 layer-averaged PV (PVU, shaded as in legend at right of panel) and $850-\mathrm{hPa}$ winds (kt, barbs); (c) $850-\mathrm{hPa}$ Geopotential height (solid contours, interval 4 dam), isotachs (kt, shaded as in legend at right of panel), and 850-hPa winds (kt, barbs); (d) 850-hPa moisture flux $\left(\mathrm{g} \mathrm{kg}^{-1} \mathrm{~m} \mathrm{~s}^{-1}\right.$, shaded as in legend at right of panel) and moisture flux vectors (arrows).....................................146

Figure 4.24. As in Fig. 4.23 except for 00 UTC 14 Jan.............................147

Figure 4.25. As in Fig. 4.23 except for 12 UTC 14 Jan............................148

Figure 4.26. As in Fig. 4.23 except for 00 UTC 15 Jan.............................149

Figure 4.27. 6-h QPFs from WRF model forecast. Valid for 6-h period ending: [(a)-(c) omitted to better match previous precipitation plots] (d) 18 UTC 13 Jan; (e) 00 UTC 14 Jan; (f) 06 UTC 14 Jan; (g) 12 UTC 14 Jan; (h) 18 UTC 14 Jan; (i) 00 UTC 15 Jan..........................................150

Figure 4.28. (a) NPVU 6-h precipitation analysis for 6-h period ending 12 UTC 14 Jan (inches, shaded as in legend at right of panels (b) and (c)); (b) as in (a) except for operational Eta model QPF; (c) as in (a) except for WRF QPF; (d) 6-h METAR observed precipitation totals (numbers, hundredths of inches) .151

Figure 4.29. WRF 12-h forecast valid 00 UTC 14 Jan 2005: (a) 250-hPa Geopotential height (solid contours, interval $12 \mathrm{dam}$ ) and isotachs (kt, shaded as in legend at right of panel); (b) 500-hPa Geopotential height (solid, interval 6 dam) and vorticity $\left(\times 10^{-5} \mathrm{~s}^{-1}\right.$, shaded as in legend at right of panel); (c) $850-\mathrm{hPa}$ potential temperature (dashed contours, interval $2 \mathrm{~K}$ ), $850-\mathrm{hPa}$ Geopotential height (solid, interval $6 \mathrm{dam}$ ), and $850-\mathrm{hPa}$ mixing ratio (shaded, interval $2 \mathrm{~g} / \mathrm{kg}$, values below $6 \mathrm{~g} / \mathrm{kg}$ omitted); (d) sea level pressure (solid contours, interval $2 \mathrm{hPa}$ ), 2-m temperature (dashed 
contours, interval $2^{\circ} \mathrm{C}$ ), and 6-h accumulated precipitation (mm, shaded as in legend at left of panel) ending 12 UTC 13 Jan....................152

Figure 4.30. As in Fig. 4.29 except for 12 UTC 14 Jan...........................153

Figure 4.31. As in Fig. 4.29 except for 00 UTC 15 Jan............................154

Figure 4.32. (a) EDAS 250-hPa Geopotential height (solid contours, interval 12 dam), isotachs ( $\mathrm{kt}$, shaded as in legend at right of panel), and divergence (dashed contours, interval $2 \times 10^{-5} \mathrm{~s}^{-1}$ ) valid 12 UTC $14 \mathrm{Jan}$; (b) as in (a) except for operational Eta model forecast; (c) as in (a) except for WRF model forecast.... 155

Figure 4.33. (a) EDAS 500-hPa Geopotential height (solid, interval 6 dam) and vorticity $\left(\times 10^{-5} \mathrm{~s}^{-1}\right.$, shaded as in legend at right of panel) valid $12 \mathrm{UTC}$ 14 Jan; (b) as in (a) except for operational Eta model forecast; (c) as in (a) except for WRF model forecast.................................156

Figure 4.34. WRF 12-h forecast valid 00 UTC 14 Jan 2005: (a) 500-950-hPa equivalent potential temperature difference (solid contours, interval $2 \mathrm{~K}$, positive values omitted) and 700-950-hPa wind shear $(10 \mathrm{kt} / \mathrm{m}$, shaded as in legend at right of panel); (b) 900-800 layer-averaged PV (PVU, shaded as in legend at right of panel) and $850-\mathrm{hPa}$ winds (kt, barbs); (c) $850-\mathrm{hPa}$ Geopotential height (solid, interval 4 dam), isotachs (kt, shaded as in legend at right of panel), and $850-\mathrm{hPa}$ winds (kt, barbs); (d) $850-\mathrm{hPa}$ moisture flux $\left(\mathrm{g} \mathrm{kg}^{-1} \mathrm{~m} \mathrm{~s}^{-1}\right.$, shaded as in legend at right of panel) and moisture flux vectors (arrows).................................157

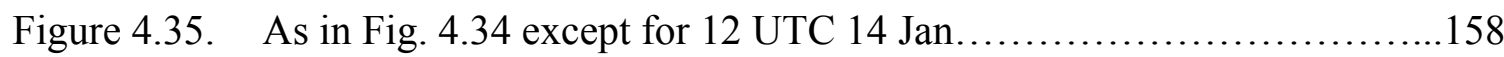

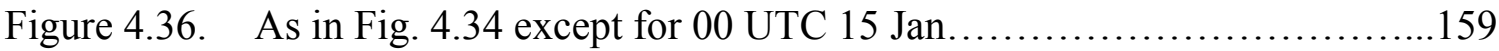

Figure 4.37. (a) 800-900-hPa PV (PVU, shaded as in legend at right of panel) and 850$\mathrm{hPa}$ parameterized latent heating (solid contours, $\mathrm{K}$ day ${ }^{-1}$ ) for $12-\mathrm{h}$ forecast valid 12 UTC 13 Jan; (b) as in (a) except for 12-h forecast valid 00 UTC 14 Jan; (c) as in (a) except for 24-h forecast valid 12 UTC 14 Jan; (d) as in (a) except for 36-h forecast valid 00 UTC 15 Jan. Solid line A-A' in (b) denotes cross-section shown in Fig. 4.40 and solid line B-B' in (c) denotes cross-section shown in Fig. 4.41......................... 160

Figure 4.38. PV budget summary for 00 UTC 14 Jan: (a) 800-900-hPa PV (PVU, shaded as in legend at right of panel) and $850-\mathrm{hPa}$ horizontal advective PV tendency (contour interval $1 \mathrm{PVU}^{\mathrm{Pay}}{ }^{-1}$, red (blue) contours correspond to positive (negative) values); (b) as in (a) except for the vertical advective 
PV tendency; (c) as in (a) except for nonadvective PV tendency; (d) as in (a) except for total PV tendency...................................161

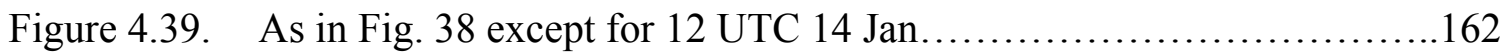

Figure 4.40. Cross section taken along line A-A' in Fig. 4.37b valid at 00 UTC 14 Jan showing parameterized latent heating $\left(\mathrm{K}_{\text {day }}{ }^{-1}\right.$, shaded as in legend at bottom of panel), PV (solid contours, PVU), and absolute vorticity vectors (arrows)..... 163

Figure 4.41. As in Fig. 4.40, except cross-section taken along B-B' in Fig. 4.37c....164

Figure 4.42. Cross section taken along line A-A' in Fig. 4.37b valid at 00 UTC 14 Jan showing PV (solid contours, PVU), absolute vorticity vectors (arrows), and section-normal wind speed ( $\mathrm{kt}$, shaded as in legend at bottom of panel) 165

Figure 4.43. As in Fig. 4.42, except cross-section taken along B-B' in Fig. 4.37c.....166

Figure 4.44. (a) Cross section taken along line A-A' in Fig. 4.37b valid at 00 UTC 14 Jan showing PV (PVU, shaded as in legend at bottom of panel), nonadvective PV tendency (contour interval $1 \mathrm{PVU}^{\mathrm{Pay}}{ }^{-1}$, solid (dashed) lines denote positive (negative) values), and non-advective PV flux vectors (arrows); (b) as in (a) except cross-section taken along B-B' in Fig. $4.37 \mathrm{c}$ 167

Figure 4.45. As in Fig. 4.44 except for advective PV tendency.......................168

Figure 4.46. (a) QGPV (solid, contour interval $4 \times 10^{-4} \mathrm{~s}^{-1}$ ) valid 00 UTC 14 Jan; (b) as in (a) except EPV (solid, contour interval .5 PVU, values below 1 PVU omitted)

Figure 4.47. Inversion summary for cyclonic QGPV anomaly at 00 UTC 14 Jan: (a) cyclonic QGPV anomaly at $900 \mathrm{hPa}$ (solid, contour interval $4 \times 10^{-4} \mathrm{~s}^{-1}$ ); (b) geopotential height (solid, contour interval 400m) and geostrophic wind recovered from inversion of cyclonic QGPV anomaly; (c) cross section of QGPV and section-normal geostrophic wind component $\left(\mathrm{m} \mathrm{s}^{-1}\right.$, solid (dashed), positive (negative) for flow into (out of) section); (d) as in (c) except for Ertel PV and observed section-normal geostrophic wind speed. 170

Figure 4.48. As in Fig. 4.47 except cross-section taken along line marked in panel (a) 
Figure 4.49. Ratio of inverted LLJ (inverted 800-hPa winds) to total LLJ (total 800-hPa winds) (percent, shaded as in legend at right) and $800-\mathrm{hPa}$ total flow (barbs, kt). 172

Figure 5.1. (a) EDAS sea level pressure (solid, contour interval $2 \mathrm{hPa}$ ) and radar mosaic imagery valid 12 UTC 31 Dec 2002; (b) as in (a) except valid 00 UTC 01 Jan 2003; (c) as in (a) except valid 00 UTC 14 Jan 2005; (d) as in (a) except valid 12 UTC 14 Jan 2005. 188

Figure A1. (a) RUC sea level pressure (solid, contour interval $2 \mathrm{hPa}$ ) and radar mosaic imagery valid 12 UTC 27 March 2005; (b) as in (a) except valid 15 UTC 27 March 2005; (c) as in (a) except valid 18 UTC 27 March 2005. 199

Figure A2. Operational Eta model 6-h total precipitation forecast ending 18 UTC 27 March 2005 (solid contours) 200

Figure A3. 6-h precipitation estimate from the National Precipitation Verification Unit (NPVU) online archive valid for 6-h period ending 18 UTC 27 March 2005 (in, shaded as in legend at left of panel).... 201

Figure A4. (a) Radar mosaic imagery valid 12 UTC 12 March 2001; (b) as in (a) except valid 18 UTC 12 March 2001; (c) as in (b) except valid 00 UTC 13 March 2001; (d) as in (a) except valid 06 UTC 13 March 2001...........202

Figure A5. Operational Eta model 6-h total precipitation forecast ending 06 UTC 13 March 2001 (in, shaded as in legend at right of panel)...................203

Figure A6. 6-h precipitation estimate from the National Precipitation Verification Unit (NPVU) online archive valid for 6-h period ending 06 UTC 13 March 2001 (in, shaded as in legend at left of panel)........................204 


\section{Introduction}

\subsection{Motivation}

It is generally acknowledged that quantitative precipitation forecasting (QPF) remains a major challenge for both numerical weather prediction (NWP) models and human forecasters (e.g., Emanuel et al. 1995; Olson et al. 1995; Fritsch et al. 1998; Zhang et al 2002; Roebber et al. 2004). It has been further shown that the forecasting of convective precipitation presents even further complication and uncertainty (e.g., Wang and Seaman 1997; Fritsch and Carbone 2004; Kain 2004).

National Weather Service (NWS) forecasters in the Southeast US acknowledge that there are a number of aspects particular to that region that can make certain types of QPF particularly challenging. One situation in which QPF has proven to be a particular challenge is characterized by intense convection upstream of a given region that significantly increases or decreases observed downstream precipitation amounts relative to the NWP model QPF.

NWS forecasters have cited examples in which NWP model forecasts significantly overpredicted QPF in portions of the Carolinas and Virginia when convection was present south or southwest of the region. Several examples of this problem are revealed in the area forecast discussions (AFDs) shown in Fig. 1. In response to such cases, conventional forecaster wisdom has evolved such that NWP model QPF in the downstream area is often reduced by human forecasters. However, while many convective scenarios may inhibit downstream precipitation, some studies have found that other upstream convection (UC) scenarios may actually result in enhanced moisture transport to the downstream region, thereby increasing precipitation amounts (Whitaker 
et al. 1988; Lackmann et al. 1998; Lackmann and Gyakum 1999; Lackmann 2002; Brennan and Lackmann 2005). Therefore, one aspect of this problem investigated in this study is the validity of the practice of reducing downstream QPF in the presence of UC.

This study analyzes events featuring UC in the Southeast US from an observational, modeling, and forecasting perspective, and examines the various ways that UC can affect downstream precipitation amounts. The main purposes of this study are to:

(i) identify the physical processes by which the downstream precipitation may be reduced or enhanced in the presence of UC;

(ii) distinguish UC cases in which downstream QPF should be reduced from those in which it should be enhanced;

(iii) understand why operational models are challenged to produce an accurate downstream forecast;

(iv) identify synoptic settings associated with different types of UC events;

(v) find ways in which human forecasters may anticipate and improve upon erroneous model forecasts, and;

(vi) investigate optimal model configurations for the representation of UC.

The current chapter outlines the major hypotheses and important background research pertaining to this problem. Chapter 2 outlines the study methodology and details the tools used for case analyses, and two major case studies are presented in Chapters 3 and 4. Chapter 5 extends the information taken from the case studies and focuses on forecasting applications, as well as providing a summary of the main conclusions and plans for future work. 


\subsection{Hypotheses - physical processes and primary scenarios}

An initial perfunctory examination of several UC cases led to the general hypothesis that there are likely three characteristic "scenarios" in which UC can occur in a manner that impacts downstream QPF in the study region. Categorized based on the speed of movement and position relative to the flow, the convective system in each of the

three general scenarios is hypothesized to affect its associated downstream environment in a different way. The possible mechanisms by which the downstream environment may be altered are listed below, followed by a description of each of the three scenarios.

\subsubsection{Mechanisms of downstream precipitation alteration}

While past events reveal that the downstream environment is often significantly altered by the presence of UC, the mechanisms by which this may occur remain undocumented. One of the main goals of this study is to determine the physical mechanisms or processes by which UC may affect the downstream precipitation field. It was hypothesized that in principle, each of the three general scenarios might demonstrate a different physical mechanism (or a different combination of mechanisms) responsible for the downstream precipitation alteration, and that QPF may also be related to a model inability to represent one or more given physical processes. Initial analyses of UC cases considered the following possible mechanisms:

(i) Moisture consumption (removal of moisture prior to its transport into the downstream area) (Fig. 1.2)

(ii) Stabilization of the downstream environment (elimination of instability in a way that reduces post-system convective precipitation, or leads to stable air being 
lifted synoptically with reduced ascent, as opposed to the lifting of unstable air) (Fig. 1.3)

(iii) Alteration of lower-tropospheric moisture transport through interruption (S1) or enhancement (S2) of the LLJ (Fig. 1.4)

(iv) Alteration of synoptic dynamics (e.g., downstream upper ridge building via latent heat release (LHR), alteration of synoptic forcing for forced ascent) (Fig. 1.5)

In addition to the processes that occur in the observed atmosphere, the various problems that NWP models demonstrate related to convective systems and related diabatic processes are also examined. Those problems related to model representation of convection may include problems with the representation of convective system structure, intensity, and propagation -- all complicated issues to be discussed further in the background research section of this chapter. Section 1.3 will concentrate on those problems related to the representation of convection in models, due to the demonstrated inability of NWP models to adequately propagate convection and the implications that this poses to the UC problem.

Diagnostics discussed in Chapters 3 and 4 identify the relative role of the potential processes of downstream environment alteration discussed above, in an attempt to determine which mechanisms tend to dominate in importance for a given scenario. Each of the three primary scenarios is outlined below. 


\subsubsection{Scenario 1}

Figure 1.6 depicts a schematic of a Scenario 1 (S1) environment. In this scenario, the UC is oriented parallel to mean flow, and the convection propagates quickly eastward relative to the primary system, in a direction perpendicular to the lower-tropospheric flow. (The means by which events are classified into a given scenario will be more clearly defined in Chapter 2.) It is hypothesized that these cases would yield decreased downstream precipitation amounts relative to the model QPF. Prior to the S1 investigation discussed in Chapter 3, any of the mechanisms (or combination of mechanisms) listed above were hypothesized to be possible candidates for affecting the downstream environment for this scenario as well as for being responsible for QPF errors. However, initial hypotheses pointed to moisture consumption (i), low-level moisture transport interruption via the alteration of the low-level wind field by the convection (iii), and numerical model error in convective system speed (v) as the most likely culprits.

\subsubsection{Scenario 2}

Figure 1.7 illustrates the environment of Scenario 2 (S2). This type of event features UC that is oriented parallel to the mean flow as in S1, but that propagates very slowly (or not at all) with respect to the primary system. It is hypothesized that a diabatically-enhanced low-level jet (LLJ) could act to enhance moisture transport (i.e. mechanism (iii)) ahead of such a system, and these cases are therefore hypothesized to yield increased downstream precipitation amounts, particularly to the north of the surface warm front and along the cold front. 


\subsubsection{Scenario 3}

A schematic of Scenario 3 (S3) is shown in Fig. 1.8. In a set-up such as this, UC is oriented perpendicular to the flow, usually aligning with the coast and/or frontal features ${ }^{1}$. While most of the present study focuses on convection over or near the Gulf of Mexico, this same idea could also apply along the NC/SC coast with respect to convection that often forms along a coastal front in southeasterly (onshore) flow. In either location, S3 cases are hypothesized to show decreased downstream precipitation, likely as a result of interrupted moisture transport (i.e. mechanism (iii)).

The following background research section will concentrate on organized convective systems and the problems related to the representation of convection in models, due to the demonstrated inability of NWP models to adequately propagate convection and the implications that this poses to the UC problem.

\subsection{Background Research}

There are several areas of background research that are important to this study. Section 1.3.1 outlines how convection is organized and how convective systems move, as well as what important feedbacks may be significant in the UC problem. Section 1.3.2 describes how convection is represented by numerical models, and the major challenges that arise in the modeling of convective systems. Section 1.3.3 discusses past research that has examined the role that a diabatically-enhanced low-level jet (LLJ) can play in

\footnotetext{
${ }^{1}$ It is believed that the convection is usually active along a warm front, although formation along a "secondary front," or a baroclinic zone located along the Gulf coast, has also been noted.
} 
moisture transport, as this will become an important component of this study in later chapters.

\subsubsection{Organized Convection}

Convective storms may be organized into three main modes: disorganized cellular convection, various types of mesoscale convective systems (MCSs), and supercells (e.g. Houze 1993, Ch. 8, p. 268). In the present study, the structures of primary interest are MCSs, a broad category than includes almost any organized multicellular convective storm, from squall lines to mesoscale convective clusters (MCCs). In particular, this study deals with baroclinic cold-season cases that exhibit strong, organized forcing.

In general, an MCS is defined as a cloud system occurring in connection with a group of thunderstorms producing a contiguous area of precipitation extending $100 \mathrm{~km}$ or more in horizontal scale in at least one direction (e.g., Houze 1993, Chapter 9). MCSs are usually organized as a result of characteristics relating to shear and instability, may be symmetric or asymmetric, and may exhibit a wide array of spatial orientations. For the UC problem examined in this study, the UC feature displays an organized structure of some kind, though the actual organization varies from case-to-case. Many studies have used a variety of diagnostic quantities such as CAPE, shear, and the bulk Richardson number to describe the character of the organization, or "convective mode" (e.g. Weisman and Klemp 1986; Bluestein 1993, see Tables 1.1 and 1.2).

For many of the cases examined here, the UC exhibited the structure of an organized squall line or a classic leading-convective-line/trailing-stratiform MCS. Texts 
such as Houze (1993), Bluestein (1993), Houze (2004), and many others describe several of the fundamental elements of MCS structure:

- The precipitation divides distinctly into a convective and stratiform region (Figure 1.9) (e.g., Houze 1993, Section 9.1.2.2)

- The convective region contains intense convective motions that produce heavy, relatively-discontinuous precipitation amounts.

- The stratiform region produces lighter and more uniform precipitation, and is due to a combination of the dissipation of older convective cells as well as a broader-sloping mesoscale layer ascent.

The fundamental differences between these two regions produce very different heating profiles, momentum budgets, and PV budgets, and therefore impact the surrounding environment quite differently (e.g., Braun and Houze 1996). As later sections will show, an important aspect of this study is MCS flow and propagation, as well as how MCS and environmental dynamics/attributes may impact the speed at which the system moves.

\subsubsection{Mesoscale Convective Systems (MCSs)}

\subsubsection{MCS downdraft dynamics}

The environmental flow in an MCS and its surrounding environment is important to the system movement, and in particular for the present study, how the downstream environment is affected. A trademark feature of the classic leading-line/trailing-stratiform MCS is its "rear-inflow jet," a layer of low- $\theta_{\mathrm{e}}$ air that enters the MCS from the rear below the trailing anvil cloud of the stratiform region and descends toward the leading 
convective line. Across the stratiform region the descent is gradual, but often accelerates downward upon approaching the convective region (e.g., Houze 2004, Section 4).

It is generally believed that thermodynamic processes (i.e. cooling by sublimation, melting, and evaporation of precipitation) drive the descending rear inflow, though several studies have found that the primary cause may be dynamical. Some findings reveal that it may be that the mid-level rear inflow jet is instead a gravity wave response to heating that occurs in the convective region (Schmidt and Cotton 1990; Pandya and Durran 1996). Still other studies have shown that other important contributions to the flow do include microphysical feedbacks (e.g., evaporative cooling), which can strengthen, if not cause, the rear inflow jet (Leary and Houze 1979; Braun and Houze 1997). Additionally, the presence of line-end vortices can also strengthen the rear inflow jet in a well-developed squall line (Skamarock et al. 1994), as can large-scale environmental flow that provides strong mid-level flow to the rear of the system (Zhang and Gao 1989).

While the rear-inflow jet plays an important role in MCS maintenance and propagation, the branches of flow in the rest of the system are also of great importance to the way in which momentum is redistributed within the MCS itself, as well as in the surrounding environment (Houze 2004, e.g., Figure 1.10). It is outside the realm of this study to fully explore these processes, but because the stratiform region can span such a large area, the momentum redistribution associated with the MCS's mesohighs and mesolows (not discussed, see Fig. 1.11) can significantly alter the large-scale momentum profile for the environment affected by the MCS. Potential implications exist for system propagation speed, as well as moisture transport in the surrounding environment. These 
implications may be of particular importance in S1 cases, as models are likely challenged to accurately represent the fast-moving UC that defines these cases.

\subsubsection{MCS movement and propagation}

MCS propagation $^{2}$ can be attributed to a number of mechanisms; primarily, cold pool dynamics and gravity wave forcing (e.g., Houze 2004, Section 7). Traditionally, cold pool propagation (i.e. the interaction of a density-current-like cold pool with convective motions) was thought to be the driving force behind all MCS propagation (e.g., Newton 1950; Rotunno et al. 1988). Cold pool propagation is simply the idea that upon arriving at the surface, negatively buoyant air (due to the diabatic cooling and precipitation loading that occurs in the downdraft) spreads out as a density current and forces conditionally unstable air ahead of the system upwards, past its level of free convection (LFC), thereby generating a new leading edge of the system. Rotunno et al. (1988) determined that a propagating system can be maintained so long as the horizontal vorticity generated by the advancing cold pool is just offset by the horizontal vorticity generated by the buoyant updraft cells forming at the cold pool boundary. This wellknown theory is often referred to as the RKW theory. However, Houze (2004) maintains that MCSs are large, complex systems, comprised of many convective cells arranged in a variety of ways, and therefore cold pool dynamics alone cannot account for all aspects of MCS propagation. Houze instead proposes that "layer overturning" (e.g., Fig. 1.12) must be part of MCS propagation theory, given a MCS's dependence on organized deep-layer

\footnotetext{
2 "Propagation" is used here to describe the general horizontal motion of an MCS. Though technically propagation and advection are distinct processes, the terms will not be differentiated in Chapter 1. MCSs generally move via a combination of both propagation and advection, and in later chapters, the two ideas will be distinguished.
} 
lifting, but acknowledges that such a theory offers no dynamical cause and effect relationship that would explain propagation.

Both cold pool dynamics and layer-overturning suggest that MCS propagation is a continuous process, though many observed systems instead appear to move in quite a discrete manner. Many studies (e.g., Doswell et al. 1996; Corfidi et al. 1996; Fritsch and Forbes 2001) have described this discrete movement in terms of vector addition, where the system motion is given by adding the large-scale environmental wind vector to a "low-level jet" vector, effectively moving the system toward higher- $\theta_{\mathrm{e}}$ air (e.g., Fig. 1.13). The dynamical explanation for discrete propagation can be explained using another popular theory - gravity wave theory, sometimes referred to as that of Thorpe, Miller, and Moncrieff (1982) (TMM), though a number of other studies have also examined this idea (e.g., Houze 1977; Fortune 1980; Houze and Betts 1981; Crook and Moncrieff 1988; Stoelinga 2003). Crook and Moncrieff (1988) found that a squall line can be initiated or maintained by an external forcing mechanism provided that the forcing mechanism provides a linearly-oriented and persistent region of lifting capable of initiating new convective updrafts. In their study, they used a model-simulated gravity-wave to illustrate the possibility that diabatic cooling and precipitation loading were less necessary to MCS maintenance relative to the external forcing due to the gravity wave. Yang and Houze (1995) also proposed that gravity waves explained the cellular nature of MCSs, suggesting that new cells are formed via gravity waves due to the gust front updraft. More recently, Carbone et al. (2002) found that the propagation speed of major convective systems is often not fully accounted for by large-scale forcing or advection by "steering winds." That study speculated that wavelike mechanisms (in either the free 
troposphere and/or the planetary boundary layer (PBL)) were fundamental contributors to the observed rates of motion. However, gravity wave propagation theory begs an important question regarding the origin of the gravity wave itself: does the MCS generate the wave, or does the wave originate from some other cause and only later become linked to the MCS? This question remains to be solved.

Along the lines of the gravity wave argument, there also exists a considerable amount of research that uses idealized model simulations to explore MCS propagation. Lin et al. (1998) examined cell development and propagation in a 2D storm simulation, finding that two distinct modes described the system: a growing mode and a propagating mode. It their study it was found that the propagating mode did exhibit gravity wave properties. A follow-up study (Lin and Joyce 2001) performed a series of sensitivity tests that showed that an advection mechanism is responsible for cell regeneration, and a gravity wave mechanism is responsible for cell propagation within the storm. Raymond and Jiang (1990) also used an idealized model to examine the maintenance of long-lived MCSs. Their results show that LHR, evaporation and melting of precipitation, and thermal radiation all contribute to MCS self-maintenance through an interaction between quasi-balanced vertical motion and the diabatic effects of moist convection. A large body of literature exists on this topic (e.g., Fovell and Tan 1998; Dudhia et al. 1987), and most results corroborate observational evidence that MCS propagation is indeed a complex, multi-faceted process. While these studies reveal important feedbacks and mechanisms of MCS growth and maintenance (particularly those that are difficult to observe or model using current operational platforms), this study will focus on the area of NWP most relevant to operational forecasting: coarser-grid models incapable of resolving features 
such as gravity waves and other forcing mechanisms typically explored by idealized studies.

There exist large bodies of research in support of both the cold-pool (or RKW) propagation theory, as well as the gravity-wave (or TMM) theory, in addition to several studies that maintain that it is a combination of these two ideas that is mainly responsible for the dynamical essence of convective propagation. Still others have proposed additional theories that may act in combination with the above effects to determine MCS motion. Many important studies have examined MCS motion from a prognostic sense as well. These forecasting-oriented studies often focus on the importance of a combination of environmental wind shear and sufficient convective instability (e.g., Emanuel 1986; Johns and Hirt 1987; Corfidi et al. 1996; Corfidi 2003), parameters which will also be examined in this study. Many of these more general, observationally-based studies have arrived at similar conclusions regarding MCS movement. For instance, it is generally accepted that the movement of an MCS is dependent on the mean flow between $850 \mathrm{hPa}$ 300hPa (Corfidi 1996) and the rate and location in which new cells form (i.e. propagation). It is also generally accepted that the propagation of an MCS depends on some or all of the following factors (though numerous other relationships have also been investigated):

- The location of the most unstable air (e.g., Johns 1984; )

- The strength and orientation of low and mid-level shear (e.g., Weisman and Klemp 1982; Johns 1993)

- The axis and the orientation of the LLJ (e.g. Corfidi et al. 1996; Corfidi 2003) 
- The depth and strength of the cold pool (particularly as it relates to the shear and moisture profile) (e.g. Rotunno et al. 1988.)

- 1000-500mb thickness patterns (e.g., Merritt and Fritsch 1984)

- The location of the strongest low-level moisture convergence (e.g., Maddox and Doswell 1982; Corfidi 2003)

Finally, MCSs include a large spectrum of convective storms, most exhibiting complex structures that do not necessarily match that of the classical leading-line/trailingstratiform system. Each different structure will have its own unique characteristics based on its organization, and this has many implications for the evolution of the system; for example, which mechanisms are fundamental contributors to its propagation, how momentum is redistributed within the system, and how the surrounding environment is altered. In the present study, many cases have been observed in which more than one upstream MCS is present in the course of an event, and that each system may have very different effects downstream. This type of multiple-MCS evolution is particularly evident in S3 cases. The implications of these variations will be further discussed in Chapter 5, as applications to observed cases are outlined.

\subsubsection{Representation of convection in numerical models}

The physical processes associated with observed convective systems are quite complex and their effects nonlinear. In a numerical model the representation of such processes is further complicated by the scale at which they take place; that is, the condensation of water vapor associated with convective motions often occurs at a subgrid scale and is therefore not resolvable by the model. In order to account for the extremely 
important effects of convective motions, the collective effects of subgridscale clouds must be accounted for in terms of the prognostic gridscale variables (Arakawa 1993). This is done using convective parameterization schemes (also termed cumulus parameterization schemes; CP schemes, hereafter).

$\mathrm{CP}$ schemes thus attempt to solve the closure problem that results from trying to add the effects of subgridscale processes to a closed set of numerical model equations. To do this, the closure assumptions must satisfy the two following requirements: 1.) the predictability of the large-scale fields must be not compromised, and 2.) they must be valid quasi-universally (Arakawa 1993). The problem of parameterization is further complicated by structures such as MCSs, as discussed above. These types of structures are partially resolved by the model, but must be partially parameterized as well (as the individual convective motions are not resolved by the model even if the total spatial scale of the system can be). The lack of a clear scale separation is a significant challenge to the success of these schemes (Molinari and Dudek 1992; Arakawa 1993; Wang and Seaman 1997; Davis et al. 2003).

There are many limitations to the extent to which $\mathrm{CP}$ schemes can accurately simulate the effects of convection in a numerical model. The reasons for this have been discussed at length in the meteorological literature, but an aspect of this problem of particular pertinence to the present study is the ability of numerical models to adequately simulate the movement of convective systems. In the previous section, the detailed processes that influence propagation are briefly described, and it is clear that it is indeed a complex set of interactions that dictates convective propagation. The representation of such interactions in a numerical model is a challenge, and current attempts to do this 
reveal many shortcomings. In a recent study of warm-season continental rainfall, Davis et al. 2003 found that significant propagation errors fundamental to CP schemes arose. The study showed that the combination of propagation errors with errors in the timing of the convection led to further degradation of QPF forecasts due to poor phase-locking of the convection with other external forcing mechanisms. Houze (2004) discusses that there remain many aspects of convective systems not yet fully understood from an observational perspective (e.g., momentum transport, propagation), and that their representation in numerical models is consequently questionable.

While CP schemes boast no shortage of shortcomings and complexities, model forecasts that are run at a high enough resolution to turn off the $\mathrm{CP}$ scheme also pose their own unique set of complications. Molinari and Dudek (1992) and Weisman et al. (1997) discuss many of these potential issues, which include an unrealistic buildup of convective instability, a delayed onset of precipitation, the presence of excessive localized precipitation amounts (usually as a result of "grid-box" thunderstorms), and the unrealistic process of gridcell overturning in large gridcells. A recent paper by Bryan et al. (2003) addresses additional issues that arise for very high resolution model runs using gridspacing on the order of $100 \mathrm{~m}-1 \mathrm{~km}$.

In the present study, several characteristics of convection will be examined to determine which pose a significant problem to downstream precipitation forecasting. Convective intensity, environmental alteration, and system propagation will all be examined within the context of each scenario. As later chapters will illustrate, the nature of the movement of the UC is a primary indicator of the type of downstream QPF modification that will likely occur. NWP model difficulty in representing convective 
system motion is a significant hurdle in achieving accurate forecasts in the presence of UC.

\subsubsection{Role of a diabatically enhanced LLJ}

Many past studies have examined the role of diabatic processes in extratropical cyclogenesis (e.g., Tracton 1973; Gyakum 1983a,b). There have also been many investigations into how convection and LHR affect the environment, particularly from a PV perspective (e.g., Davis and Emanuel 1991; Davis 1992a; Stoelinga 1996; Brennan and Lackmann 2005; Mahoney and Lackmann 2005). As alluded to in the previous section, one of the main goals of the present study is to investigate how the presence of UC might limit or enhance the downstream precipitation amount, and to test the validity of the conventional forecaster wisdom that states that UC unconditionally reduces downstream QPF.

Lackmann (2002) examined a cold front in which a diabatically-enhanced LLJ led to increased moisture transport ahead of the system, thereby altering the downstream environment. The increase in moisture aided precipitation generation along and ahead of the system, rendering the model QPF a forecast "miss." By performing a PV budget, the LHR from the cold-frontal rainband was found to be a significant contributor to the LLJ via the cyclonic circulation associated with its LHR-induced low-level PV maximum. Because models often having trouble representing LHR and precipitation-related diabatic processes, such a feedback mechanism may have major implications for downstream precipitation forecasts. 
The working hypothesis in the present study is that a similar mechanism as was found in Lackmann (2002) may also play a role in enhancing the S2 downstream precipitation, given a favorable environment. Chapter 2 will discuss the experiments undertaken to examine this hypothesis, as well as the complete methodology adopted for this study. 
AREA FORECAST DISCUSSION

NATIONAL WEATHER SERVICE GREENVILLE-SPARTANBURG SC

943 PM EST MON DEC 302002

... GFS HAS BEEN CONSISTENTLY THE FASTEST SOLUTION...BUT $12 Z$ RUN HAS

TRENDED

BACK TOWARD ETA TIMING. ALL SOLUTIONS BRING SOME MEASURE OF DEEP GULF

MOISTURE AND STRONG ISENTROPIC LIFT AND UPSLOPE FLOW EITHER SIDE OF

$12 Z$ WED. WILL THUS SPLIT BEST POPS AROUND THIS POINT. GIVEN MOIST SOIL CONDITIONS WILL ISSUE A SPECIAL WEATHER STATEMENT ADDRESSING POTENTIAL FOR HEAVY RAIN LATE TUE NIGHT/EARLY WED...BUT IT IS TOO EARLY TO CONSIDER A FLOOD WATCH GIVEN TIMING DIFFICULTIES AND POTENTIAL OF UPSTREAM CONVECTION TO ROB SYSTEM OF MOISTURE.

AREA FORECAST DISCUSSION

NATIONAL WEATHER SERVICE GREENVILLE-SPARTANBURG SC

300 PM EST TUE DEC 312002

... A VERY CHALLENGING PRECIPITATION AMOUNT FORECAST HAS SET UP AS UPSTREAM CONVECTION MAY BE LARGELY ROBBING THE MOISTURE SOURCE.

HOWEVER...DEVELOPING SE FLOW OVERNIGHT SHOULD DISSIPATE THE STABLE SFC RIDGE ACROSS THE REGION...ALLOWING CONVECTION TO INCREASE OVER THE WRN CAROLINAS AND NE GA.

AREA FORECAST DISCUSSION

NATIONAL WEATHER SERVICE RALEIGH NC

910 AM EST SUN MAR 272005

.UPDATE. .

LATEST RADAR TRENDS SHOW RADAR RETURNS GREATLY DIMINISHING AS THEY MOVE INTO MOVE STABLE AIRMASS OF THAT OF THE "CAROLINA WEDGE". LATEST METARS ACROSS THE SOUTHERN PLAINS/COASTAL AREAS SHOWING BACKING OFF WINDS WITH ONSET OF PRECIP...WHICH SHOULD REINFORCE IN-SITU DAMMING. EVEN OF MORE SIGNIFICANCE COULD BE CUT-OFF OF MOISTURE FEED FROM LARGE AREA OF DEEP CONVECTION ACROSS THE DEEP SOUTH... PERPENDICULAR TO THE MEAN FLOW. THE GFS IS VERIFYING MUCH BETTER THAN THE NAM AT SYNOPTIC HOUR...WITH HOLDING ON TO COOLER WEDGE AIRMASS AND TWO DISTINCT AREAS OF PRECIP ACROSS THE AREA. WITH ALL THAT SAID...LOOKS LIKE WIDESPREAD SIGNIFICANT PRECIP WILL BE DELAYED UNTIL THIS EVENING WHEN WARM FRONT MOVES INTO THE AREA.

Figure 1.1. Area Forecast Discussions from National Weather Service forecast offices referencing the challenge that UC poses to the downstream QPF. 


\section{(i) Consumption}

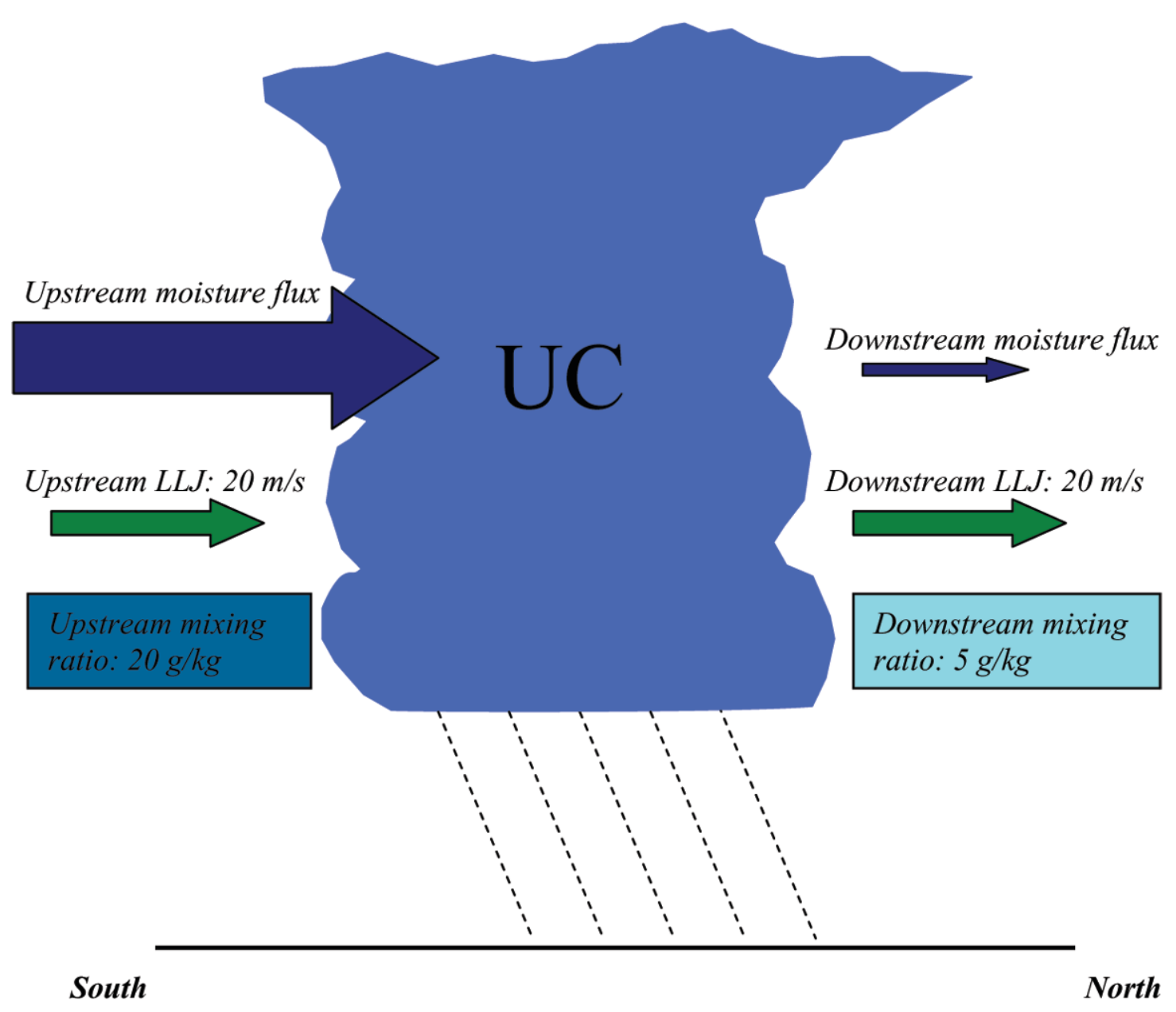

Figure 1.2. Schematic of physical mechanism (i), consumption. 


\section{(ii) Stabilization}

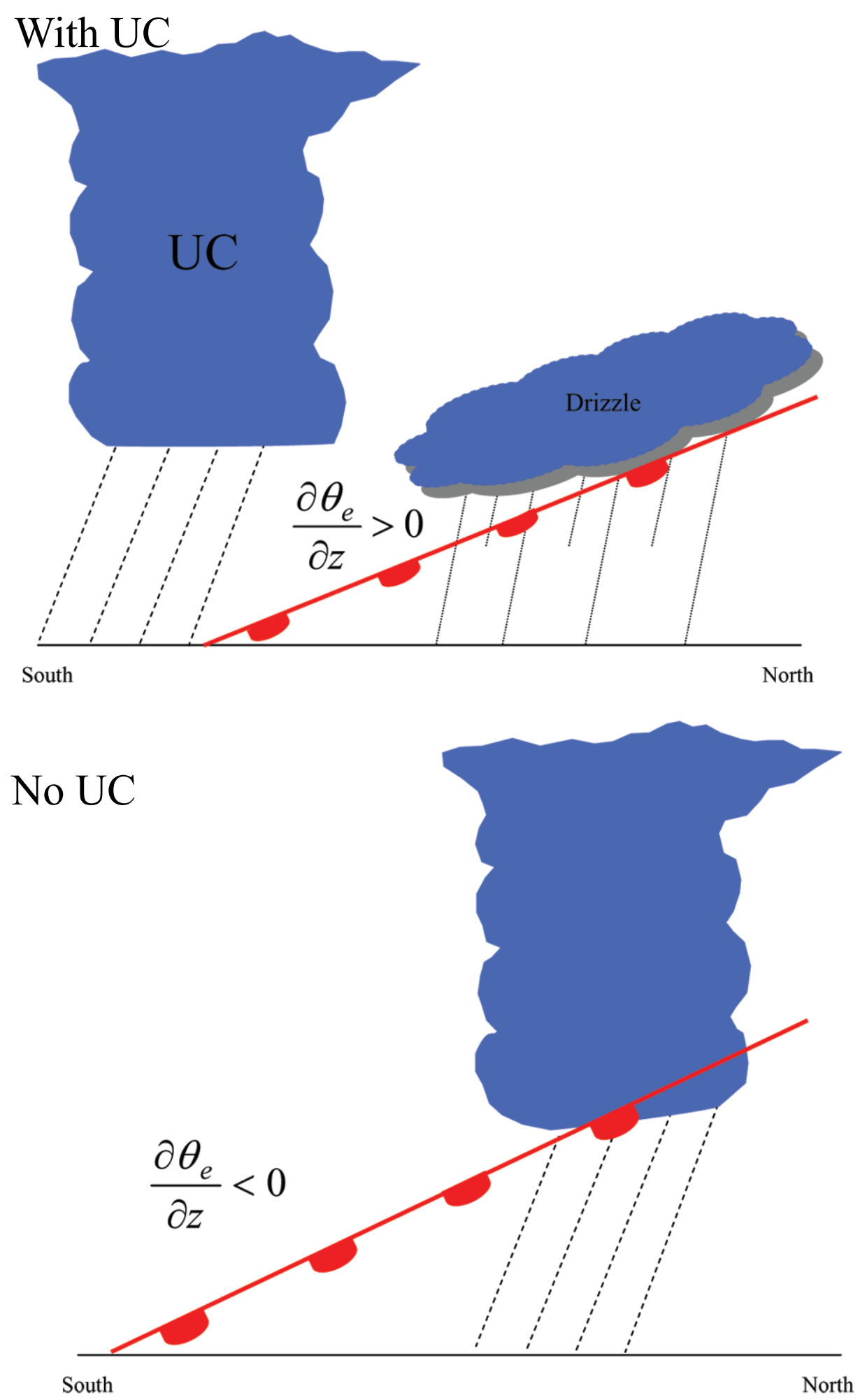

Figure 1.3. Schematic of physical mechanism (ii), stabilization. 
(iii) Alteration of lower-tropospheric moisture transport
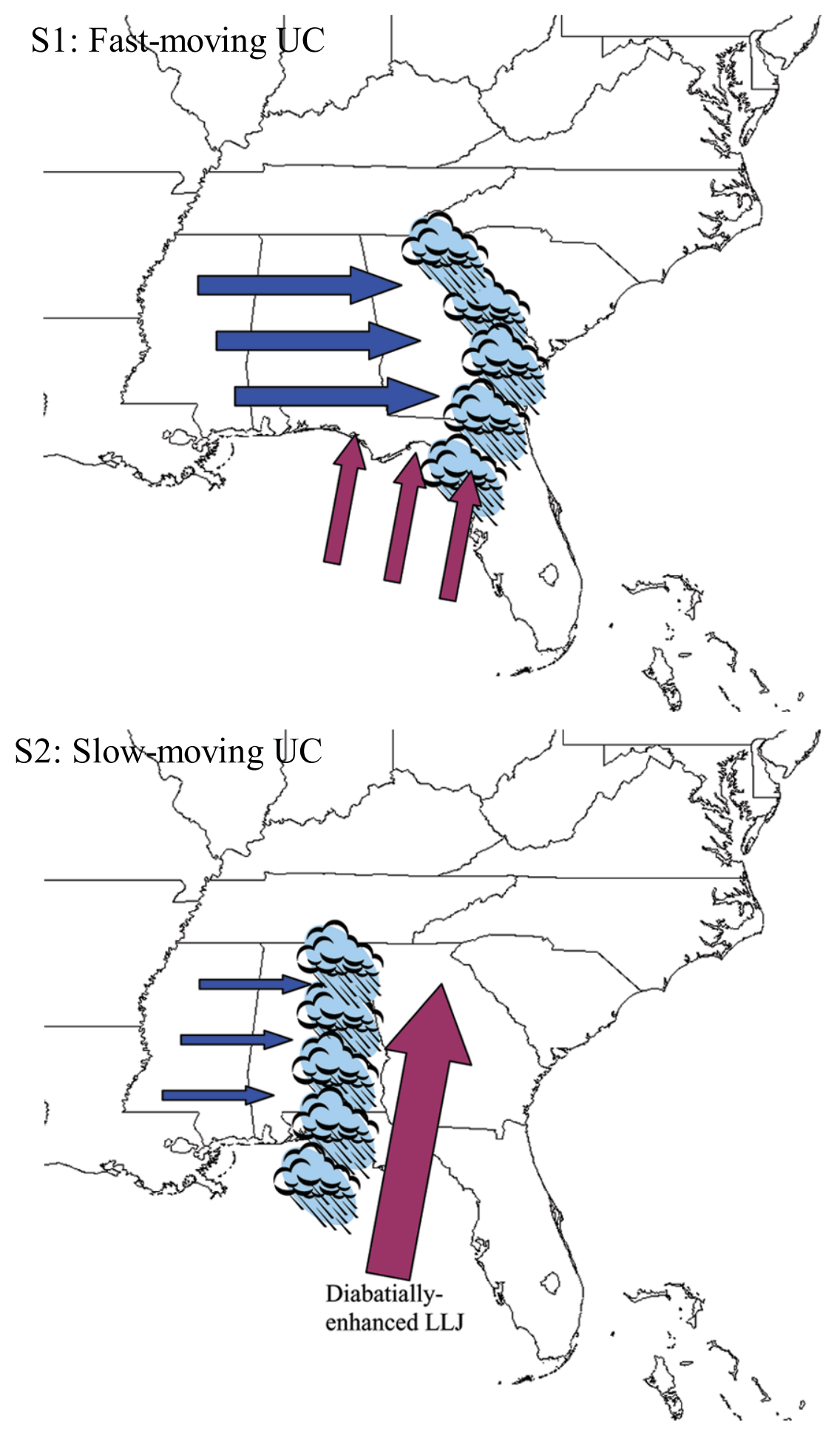

Figure 1.4. (a) Schematic of physical mechanism, (iii) interruption of lower-tropospheric moisture transport (S1); (b) as in (a), except for S2, showing the enhancement of lower-tropospheric moisture transport. 


\section{(iv) Alteration of synoptic dynamics}

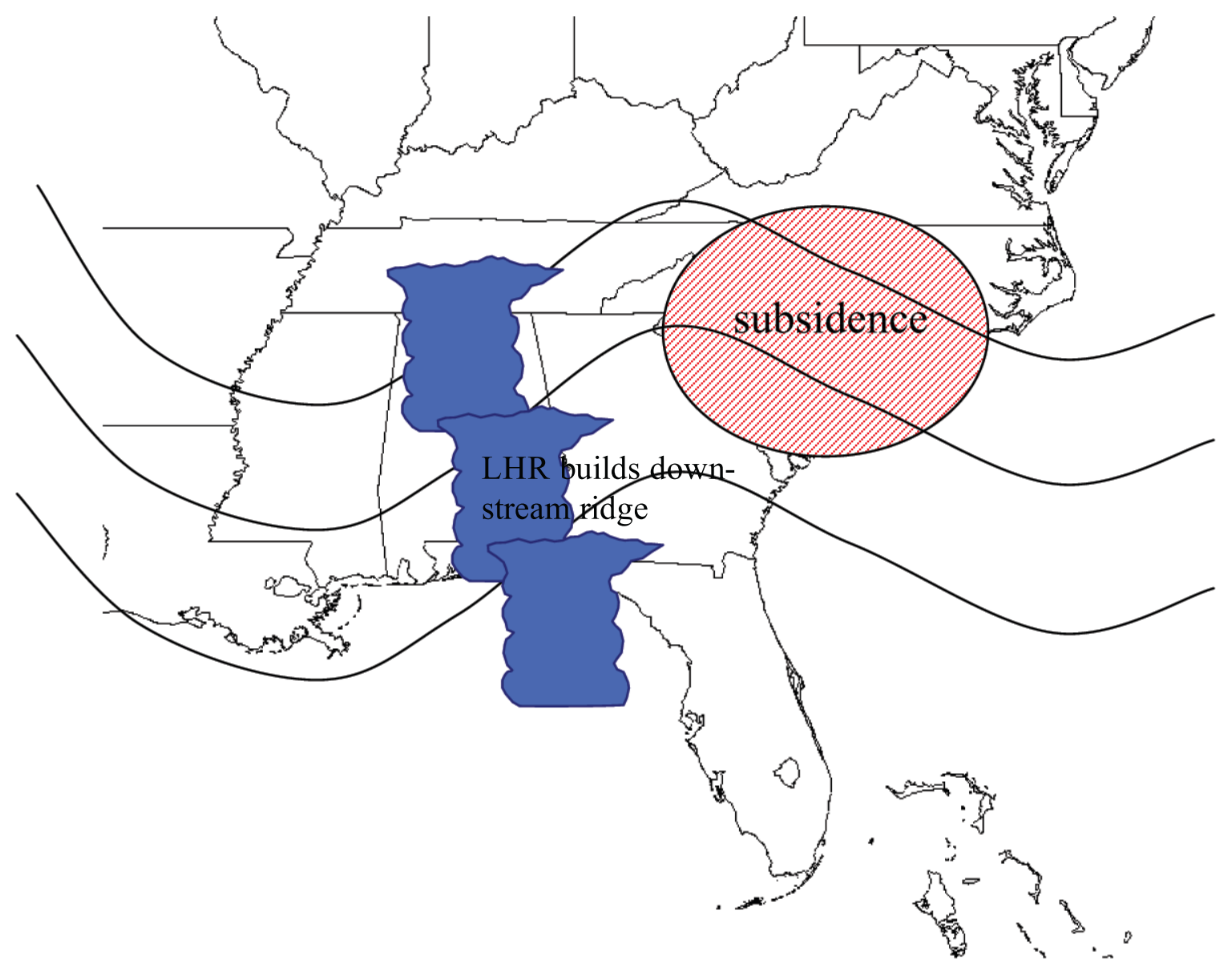

Figure 1.5. Schematic of physical mechanism (iv) alteration of synoptic dynamics. 


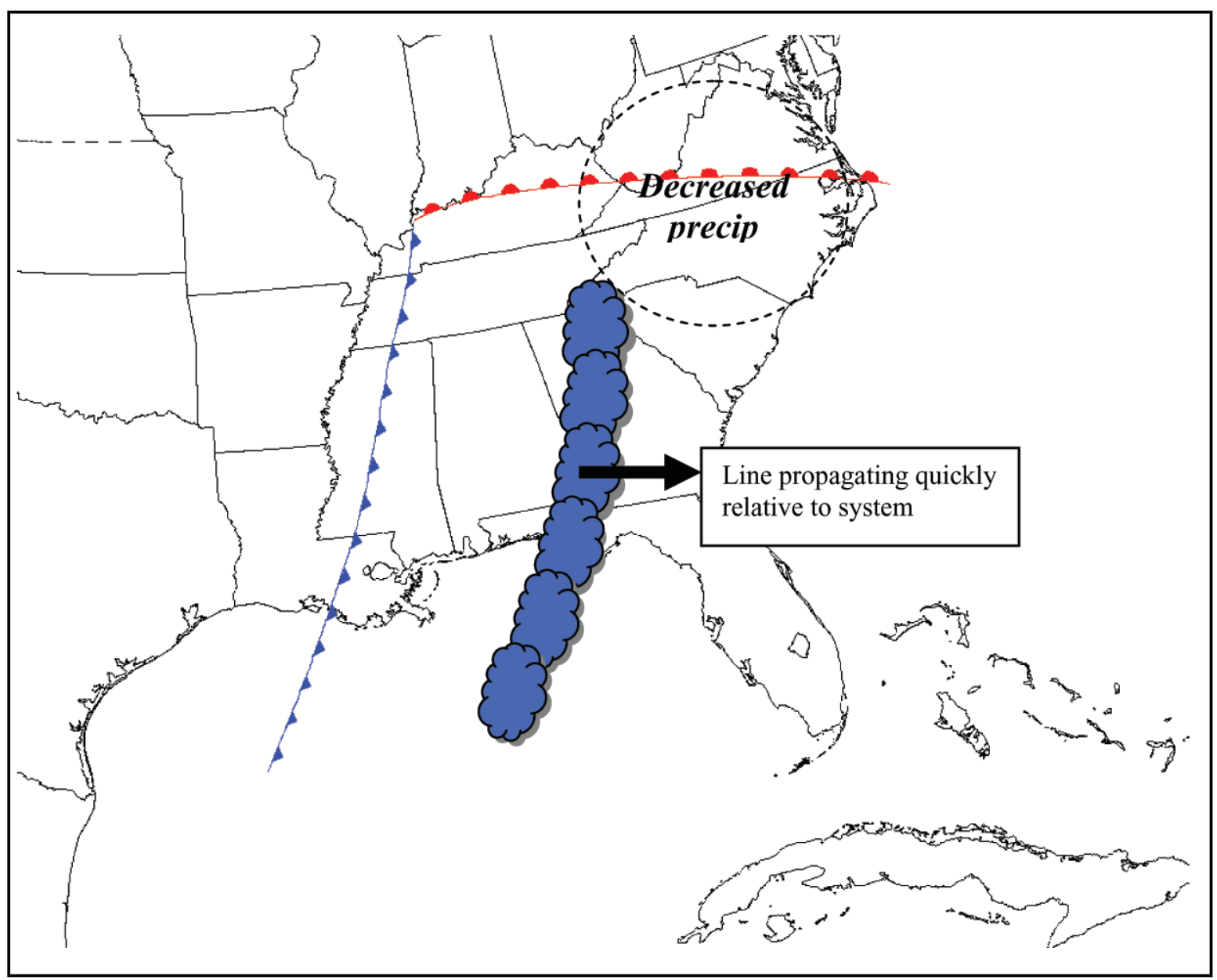

Figure 1.6. Schematic of Scenario 1 (S1). 


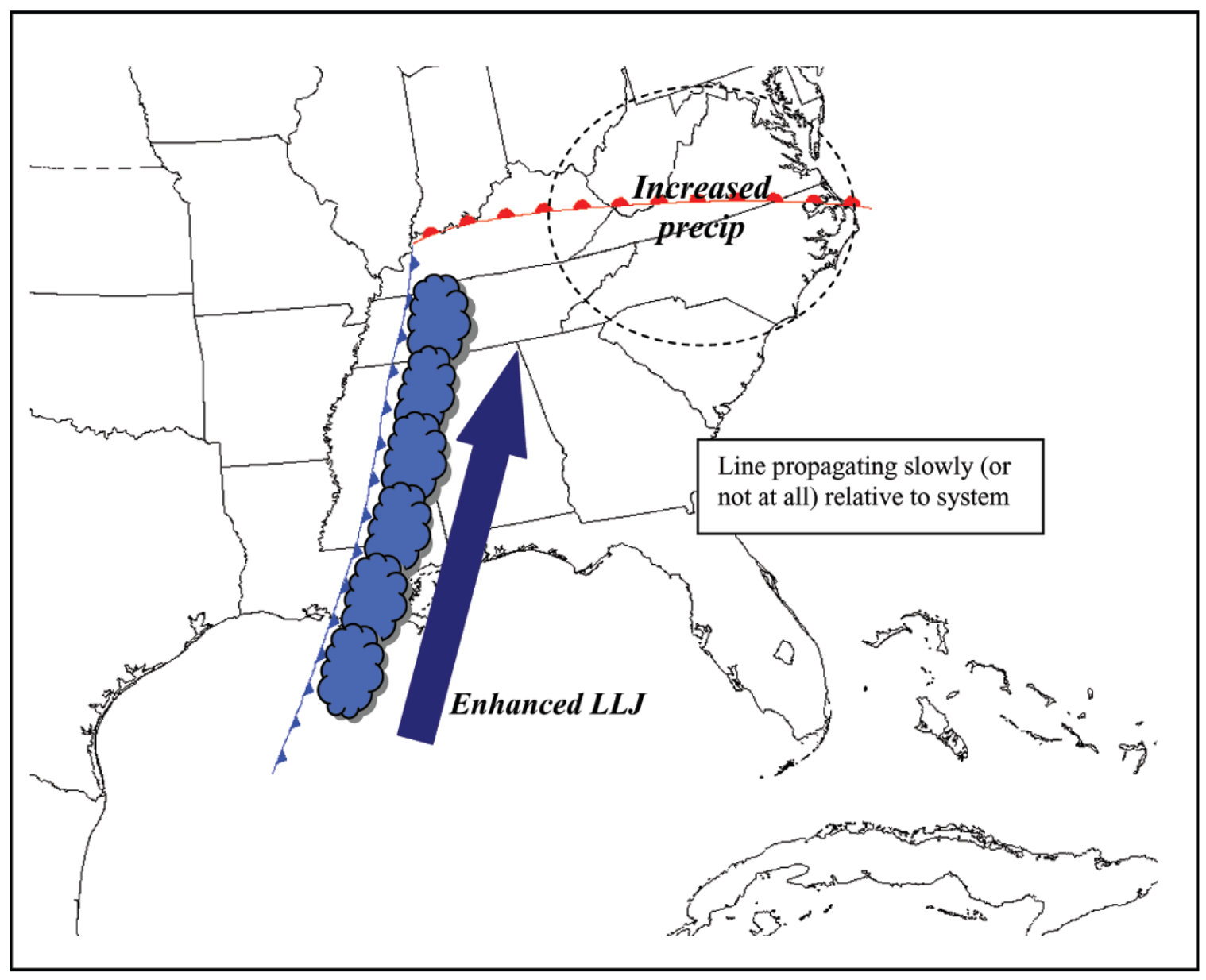

Figure 1.7. Schematic of Scenario 2 (S2). 


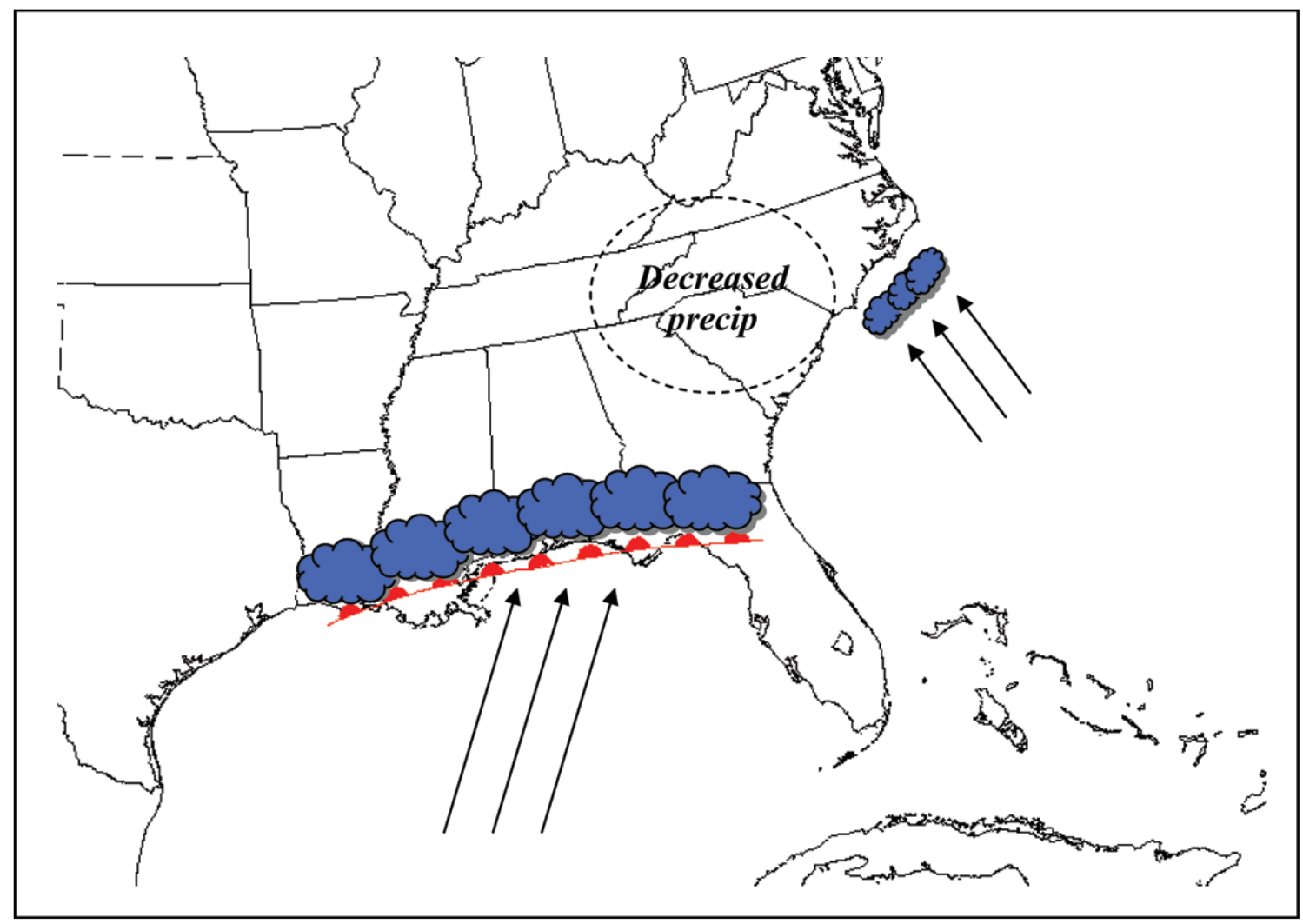

Figure 1.8. Schematic of Scenario 3 (S3). 
(a)

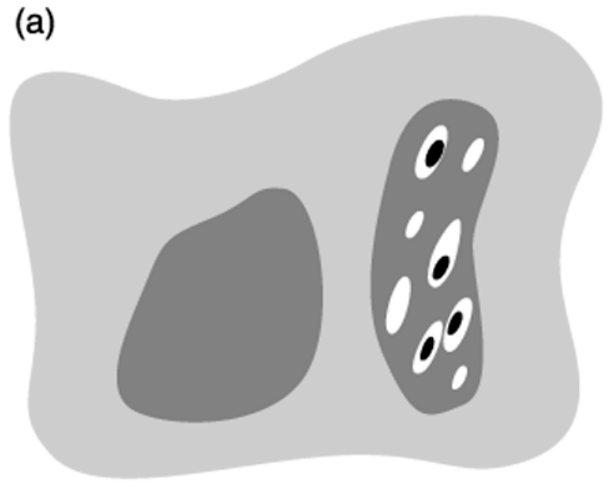

(b)

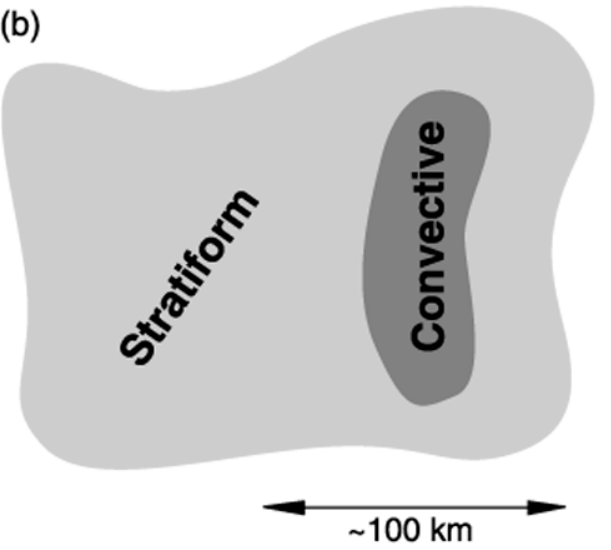

Figure 1.9. (a) Idealization of a horizontal map of radar reflectivity (b) divided into convective and stratiform regions (from Houze 1997.) 


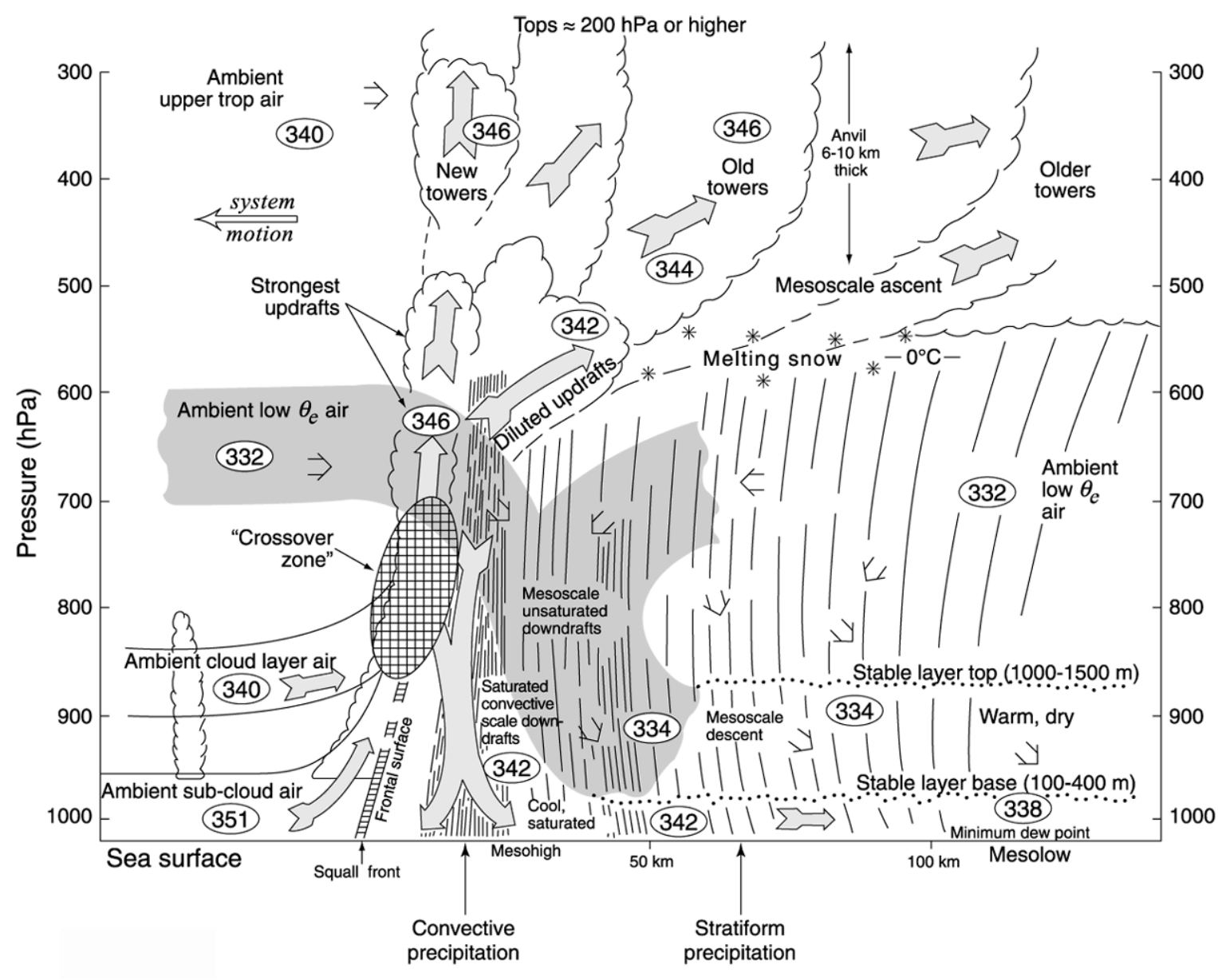

Figure 1.10. Idealization of a mesoscale convective system with leading-line/trailing stratiform structure, showing branches of flow and position of surface mesohighs and mesolows (from Houze 2004 after Zipser 1977). 


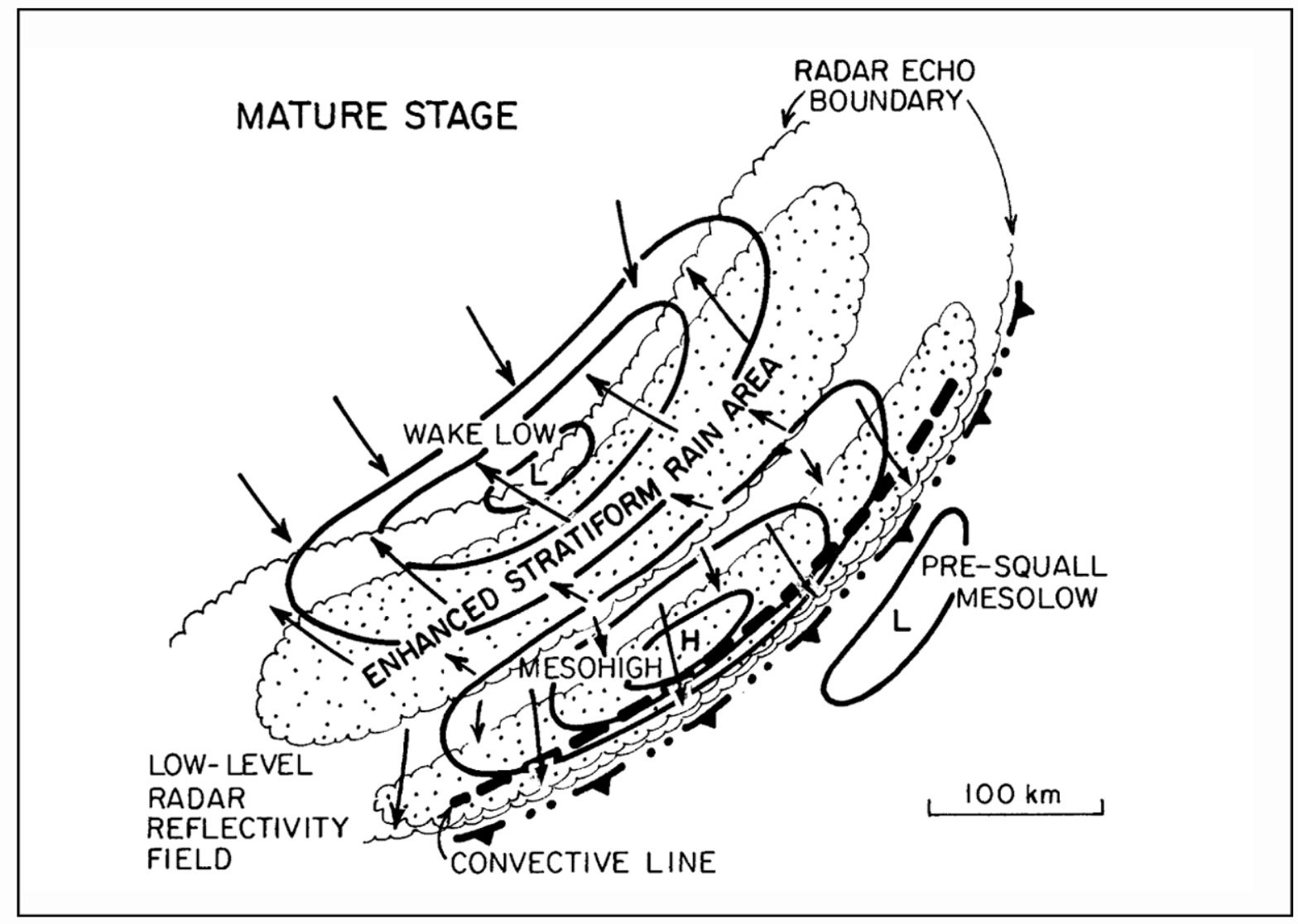

Figure 1.11. Schematic of a mature squall system. Surface pressure is contoured, vectors represent surface wind, and significant regions of precipitation are stippled (from Haertel and Johnson 2000 after Johnson and Hamilton 1988). 


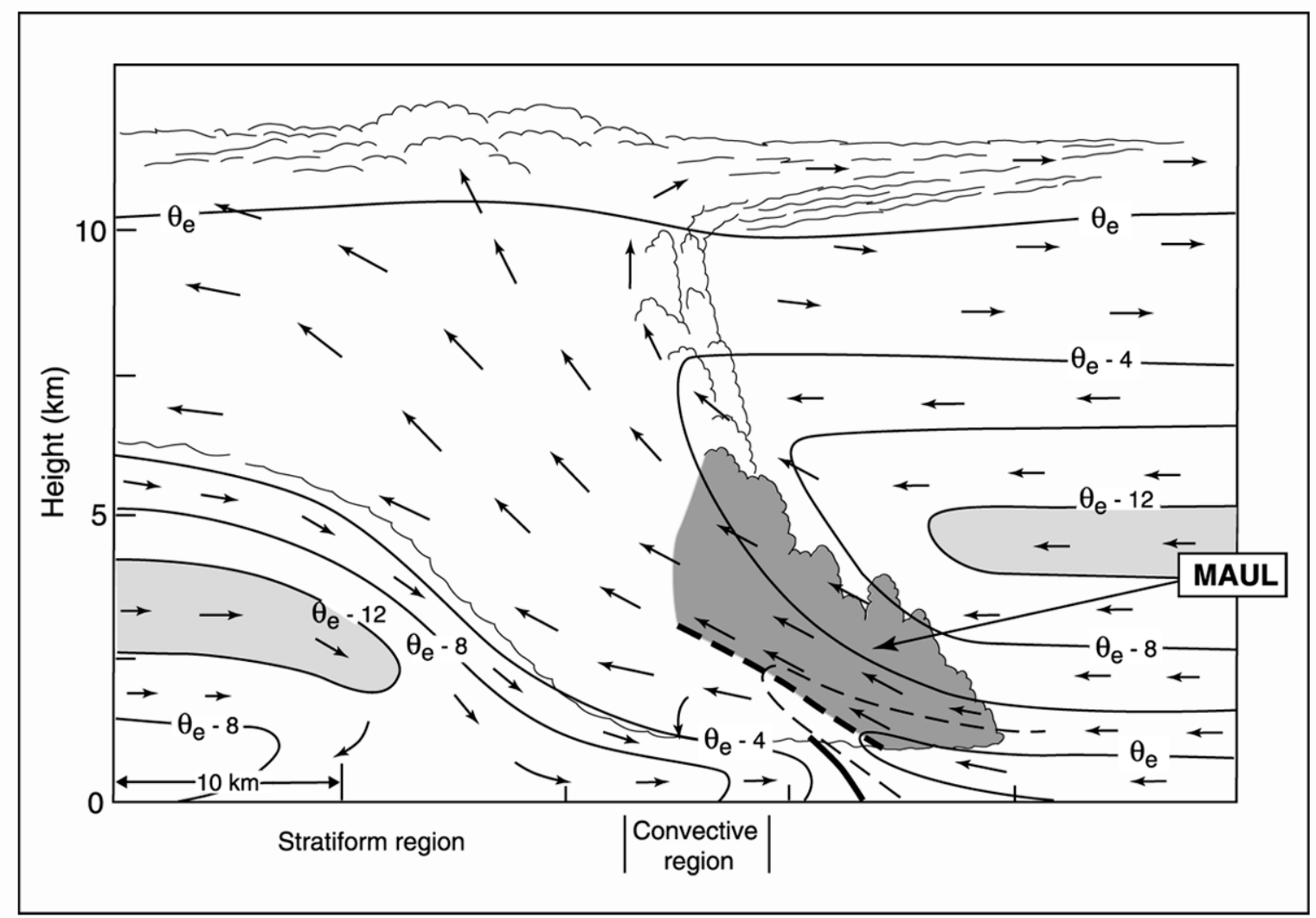

Figure 1.12. Idealized cross section through slab convective overturning. Flow vectors are system relative, scalloped lines indicate cloud boundaries, solid lines are e contours every $4 \mathrm{~K}$ (thin dashed line is an intermediate contour, and bold dashed line marks axis of highest values), bold solid line indicates outflow boundary or frontal zone, light shading highlights middle level layer of low- e air, and dark shading depicts the moist absolutely unstable layer (MAUL) (from Houze 2004 after Bryan and Fritsch 2000.) 

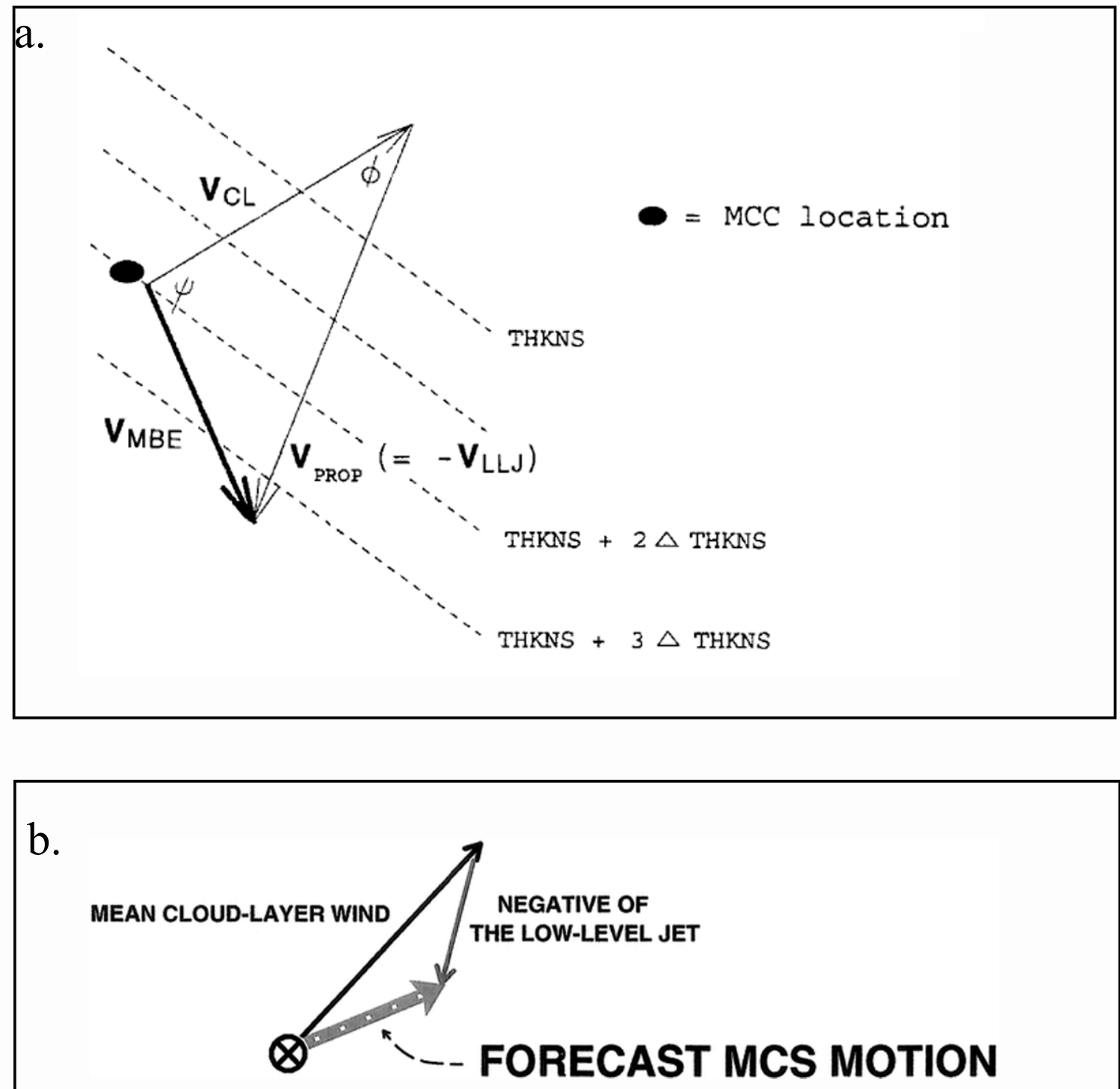

Figure 1.13. (a) Conceptual model of MCS movement (VMBE) as the vector sum of the mean flow in the cloud layer (VCL) and the propagation component (VPROP). The magnitude and direction of VPROP are assumed to be equal and opposite to those of the low-level jet (VLLJ). The angles and are used to calculate VMBE given observed values of VCL and VLLJ. Dashed lines (labeled THKNS) indicate a typical relationship of the 850-300-mb thickness pattern to the environmental flow and MBE movement during MCC events (from Corfidi 1996); (b) Schematic of the original vector technique, with MCS core motion (thick dotted arrow) expressed as the vector sum of 1) advection of cells by the mean cloud-layer wind (arrow pointing to upper right) and 2) cell propagation directed into the low-level jet (arrow pointing to bottom of page). MCS centroid is depicted by the cross symbol (from Corfidi 2003 after Corfidi et al. 1996). 


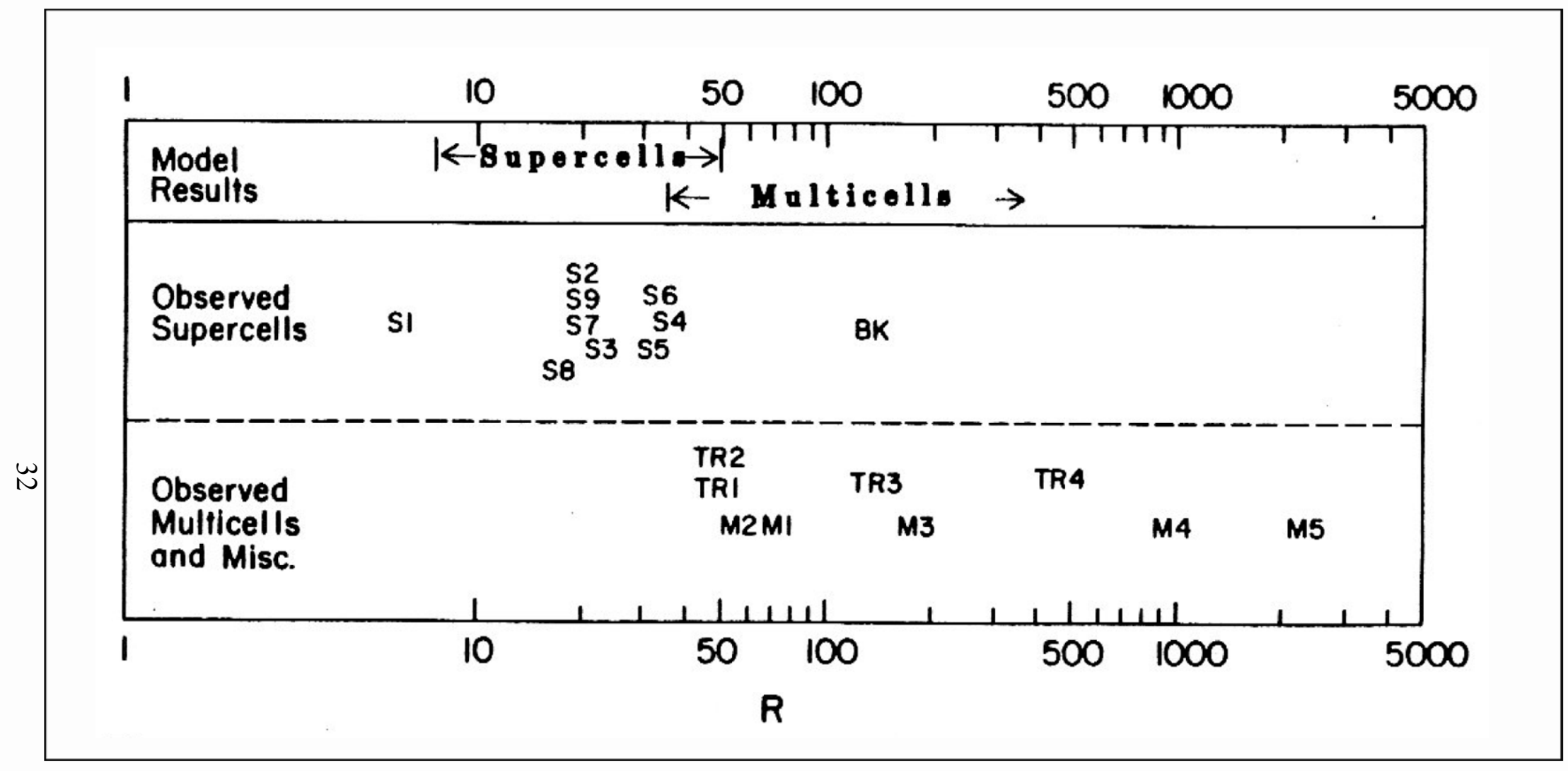

Table 1.1. Richardson number $\mathrm{R}$ as calculated for a series of documented storms. Model results are summarized at the top of the figure. S1, S2, .., S9 represent supercell storms; M1, M2, .., M5 represent multicell storms; TR1, TR2,.., TR4 represent tropical cases (from Weisman and Klemp 1986 after Weisman and Klemp 1982). 


\begin{tabular}{|c|c|c|c|}
\hline CAPE ${ }^{\text {Vertical shear }^{a}}$ & $\begin{array}{l}\text { Weak } \\
\leqslant 15 \mathrm{~m} \mathrm{~s}^{-1}\end{array}$ & $\begin{array}{c}\text { Moderate } \\
\sim 15-25 \mathrm{~m} \mathrm{~s}^{-1}\end{array}$ & $\begin{array}{l}\text { Strong } \\
\geq 25 \mathrm{~m} \mathrm{~s}^{-1}\end{array}$ \\
\hline $\begin{array}{l}\text { Low } \\
\left(500-1000 \mathrm{~J} \mathrm{~kg}^{-1}\right)\end{array}$ & Ordinary cell & $\begin{array}{l}\text { Ordinary } \\
\text { cell/supercell }\end{array}$ & $\begin{array}{l}\text { Ordinary } \\
\text { cell/supercell }\end{array}$ \\
\hline $\begin{array}{l}\text { Moderate } \\
\left(\sim 1000-2500 \mathrm{~J} \mathrm{~kg}^{-1}\right)\end{array}$ & Ordinary cell & 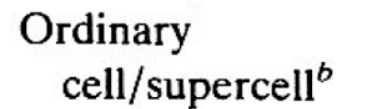 & Supercell $^{b}$ \\
\hline $\begin{array}{l}\text { High } \\
\left(\geqq 2500 \mathrm{~J} \mathrm{~kg}^{-1}\right)\end{array}$ & Ordinary cell ${ }^{b}$ & $\begin{array}{l}\text { Ordinary } \\
\text { cell }^{b} / \text { supercell }^{b}\end{array}$ & Supercell $^{b}$ \\
\hline
\end{tabular}

${ }^{a}$ Over lowest $6 \mathrm{~km}$.

${ }^{b}$ Storms in which severe weather is likely. Vertical shear is measured by the length of the hodograph of the environmental winds from the surface to $6 \mathrm{~km}$ AGL (small-scale curves and loops are not counted). Supercells can occur even in environments of low CAPE if there is low CIN and if the environment is so moist that entrainment of environmental air does not weaken the updraft significantly. Severe weather is likely in storms produced in an environment of moderate-high CAPE regardless of storm type because the updrafts can be strong (based upon numerical simulations by M. Weisman, NCAR).

Table 1.2. Storm type as a function of vertical shear and CAPE (from Bluestein 1993, Table 3.2). 


\section{Methodology}

This chapter describes the basic methodology that was followed for subsequent sections of this study. The first section describes the brief climatology that was performed prior to the selection of case study events, the second section details the model runs performed for each case study, and the last section describes the PV budget and PV inversion methodology used in Chapters 3 and 4.

\subsection{Eta model QPF error climatology and case selection}

A somewhat cursory $\mathrm{Eta}^{1}$ model QPF error climatology was generated by evaluating the Eta-model ${ }^{2}$ 24-h precipitation forecasts (valid from forecast hours 12-36) against the North American Regional Reanalysis (NARR) dataset (Mesinger et al. 2005) for the years $2000-2003$.

The NARR dataset provides analyses based on Eta Data Assimilation System (EDAS) analyses (Rogers et al. 1995; Rogers et al. 1996). The data are interpolated onto a grid with 32-km gridspacing, and also incorporates a wide variety of observations (Zaptocny et al. 2000). The QPF error climatology performed here relied on the NARR precipitation analyses, which come primarily from Eta model output that is nudged toward observed rain gauge measurements. There have been several recent efforts to assess the accuracy of this dataset (Mesinger et al., submitted), but such issues were not addressed in a great amount of detail for the present study, as only a "ballpark" estimate was needed, due to the fact that only the largest model errors were examined.

\footnotetext{
${ }^{1}$ The NCEP Eta model was renamed to the North American Mesoscale (NAM) model on 25 Jan 2005.

${ }^{2}$ The Eta forecasts used were interpolated to the World Meteorological Organization (WMO) 211 grid (approximately $80-\mathrm{km}$ grid spacing)
} 
For the QPF error climatology, precipitation totals (both the model forecast and the "actual" precipitation total) were averaged over a $200-\mathrm{km}$ radius centered near Raleigh, NC (Fig. 2.1). Model errors were assessed each day of the cold season (defined as 1 Oct -31 Mar) for a 4 -year period $(2000-2003)$. Based on the differences in the predicted and observed precipitation amounts, the events were then classified as hits, misses, or false alarms. Misses were defined by an area-averaged model under-prediction of $5 \mathrm{~mm}$ or more, false alarms were defined by a model over-prediction of $5 \mathrm{~mm}$ or more, and the remaining cases were labeled "hits." The events were ranked by the magnitude of the model error, and the cases with error magnitudes exceeding $20 \mathrm{~mm}$ were examined further (e.g. Table. 2.1). These cases were further examined for the presence of UC using the National Climatic Data Center's online NEXRAD National Mosaic Relfectivity Image archive, and of those that displayed UC (defined as a contiguous region of radar echoes in excess of $50 \mathrm{dBz}$ in the upstream area), two cases were selected for in-depth case study examination, one representing S1 and the other representing S2.

The case chosen to represent S1 was selected based primarily on its poor model QPF (operational NWP forecasts over-predicted actual downstream precipitation amounts by an area-averaged amount of $35 \mathrm{~mm}$ ), as well as its likeness to the $\mathrm{S} 1$ schematic. (The schematics for S1, S2, and S3 were developed using a combination of forecaster conventional wisdom as well as the findings of the QPF error climatology discussed above.)

Likewise, the case chosen to exemplify S2 displayed an erroneous model QPF, and represented the S2 schematic adequately well. The S2 case is a recent one (13 - 14 Jan 2005), and was chosen following the real-time realization of its representativeness of 
S2. Therefore NARR precipitation analyses are not yet available for the event, and thus the area-averaged QPF error cannot be quantified at this time. However, as Chapter 4 will show, the QPF error was significant for this case, though notably less pronounced than that seen in the S1 case study (Chapter 3.)

S3 cases proved to be difficult to pinpoint, as the QPF error climatology did not reveal many large model QPF errors associated with cases resembling S3 (in spite of personal communication with forecasters that suggested that cases resembling S3 do occur with significant frequency). After examining various recent cases that were identified by the similarity of their radar signals to the S3 schematic, it became apparent that many of the cases that initially fit the $\mathrm{S} 3$ schematic often evolve into a setup that is more representative of $\mathrm{S} 1$, or at least no longer resembles the scenario originally hypothesized to be S3. Following this transition, if the system evolves into an S1 or S2 system, then the UC will be active in the warm sector of the system, rather than along the initial warm front as in the earlier stages of system evolution (i.e. a S3 set-up). To better focus this investigation, it was decided to limit this study to those cases featuring UC occurring in the warm sector of the cyclone, rather than all types of UC. Therefore, based on the results of the QPF error climatology, no S3 case study was chosen, but the implications of cases in which the scenario type evolves during the event will be discussed in Chapter 5.

\subsection{Numerical simulations and forecasts}

Both the fifth-generation version of the Pennsylvania State University-National Center for Atmospheric Research mesoscale model (MM5) and Weather Research and 
Forecast (WRF) were used to perform model experiments as well as generate physicallyconsistent datasets with which to better analyze the chosen case studies. Much of the observational and operational model data used to initialize, evaluate, and display the output of these model runs was received via the Unidata program and displayed using the General Meteorological Package (GEMPAK, desJardins et al., 1991). Also, radar imagery was obtained from Weather Surveillance Radar-1988 Doppler (WSR-88D) sites.

This section details the model setup and specifications that were used in the model experiments and diagnostics discussed in Chapters 3 and 4.

\subsubsection{Mesoscale models}

\subsubsection{PSU-NCAR mesoscale model (MM5)}

At the outset of this investigation MM5 was used for several preliminary model runs on the S1 case study. MM5 is a non-hydrostatic model that features numerous options for model physics, as well as the capability for nesting multiple domains. An overview of the model and the prognostic model equations can be found in Dudhia (1993) and Grell et al. (1994). Version 3.5 of MM5 was used for the model run that is discussed here. While the WRF model was eventually chosen over the MM5 for this study, the preliminary model runs did serve an important role in initial modeling efforts.

The model run that is briefly presented in Chapter 3 was a $20-\mathrm{km}$ run that was initialized at 12 UTC 30 Dec using Eta 212 model analyses (Fig. 2.2). The Kain-Fritsch CP scheme was used, and other parameterizations included the Reisner microphysics scheme, the Eta-Mellor-Yamada planetary boundary layer (PBL) scheme, the Oregon State University (OSU) land surface model (LSM), and the Dudhia radiation scheme. 
Lateral boundaries were updated by Eta 212 analyses every 12 hours, making this a model simulation rather than a forecast. Therefore, there are two important aspects of this model run that make it somewhat incongruous the other runs used in this study: (i) it was initialized 12 hours earlier than the other runs (12 UTC 30 Dec as opposed to 00 UTC 31 Dec), and (ii) it is a model simulation, not a model forecast. Were this run used to make any important conclusions, these would have become significant issues, but as Chapter 3 will show, the output from this run is only used to provide a benchmark against which to compare the WRF model forecasts described below.

\subsubsection{Weather Research and Forecast (WRF) model}

WRF is a flexible mesoscale modeling system that was jointly developed by NCAR and NCEP and possesses numerous options for model physics and configuration. An overview of the WRF model and its structure can be found in Michalakes et al. (2001). For all of the WRF model runs discussed in the present study, Version 2.0.3.1 of the Advanced Research WRF (ARW) dynamical core was used (see Skamarock et al. 2005 for further detail).

WRF was the modeling system chosen for this study for several reasons. First, the WRF architecture features superior finite-differencing and more efficient computation relative to other models such as Eta and MM5, and can therefore be run on finer-mesh domains with less computational expense. Second, when run at sufficiently high resolution to omit the use of a $\mathrm{CP}$ scheme (i.e. a "convection permitting" run), WRF has been purported to represent convection and the movement of convective systems in a superior manner to other models (Done et al. 2004), aspects that will be shown to be of 
great significance to this study. Finally, WRF is currently scheduled to be implemented operationally in Spring 2006, and due to the operational nature of this project, it is of greatest benefit to the forecasting community to use a model that is most representative of what will be most widely used in the near future. Details of the individual model simulations will be presented in the sections that follow.

\subsubsection{Mesoscale model runs: WRF S1 case study}

Many preliminary model runs were performed for the S1 case study, beginning with several different model runs using MM5, as well as various experimental runs using the WRF model. These runs were performed with the goal of obtaining an accurate model forecast for the quickly-propagating squall line that characterized this case. The model run that adequately achieved this goal was an explicit $4-\mathrm{km}$ model forecast using the WRF model. The model domain encompassed an area over the Southeast US, and included as large an area of the Gulf of Mexico as could be handled by the computing system (Fig. 2.3). As the run was explicit, no CP scheme was employed, and all precipitation was thus generated at the grid scale. The gridspacing was $4 \mathrm{~km}$, and the run was initialized at 00 UTC 31 Dec 2002 and run for 48 hours out to 00 UTC 2 Jan 2003. Eta 211 model analyses and forecasts were used as initial conditions and boundary conditions, respectively, and boundary conditions were updated every 6 hours. Parameterizations used included the Ferrier microphysics scheme, Rapid Radiative Transfer Model (RRTM) longwave radiation scheme, Dudhia shortwave radiation scheme, the Noah land-surface model, and the Yonsei University (YSU) PBL scheme. 
Half-degree SST data were used. This model run and its results will be discussed in detail in Chapter 3.

Additionally, for the purpose of performing an Ertel PV budget (detailed in the following section), a WRF model run was also performed for this case using many of the same characteristics as above, except with coarser grid-spacing. This run was nearly identical to the 4-km run, except that it used the KF CP scheme, and its grid-spacing was $20 \mathrm{~km}$ (Fig. 2.4). The PV budget performed using this model forecast output is also discussed in Chapter 3.

\subsubsection{Mesoscale model runs: WRF S2 case study}

The WRF model was also used to generate a model forecast that adequately represented the system examined in the S2 case study. For this case, a coarser-resolution 20-km WRF forecast sufficed, and satisfactorily represented the system so that diagnostic experiments could be performed with the dataset.

For the S2 model run the domain was larger and $20-\mathrm{km}$ gridspacing was utilized (Fig. 2.5). The run was initialized at 12 UTC 13 Jan 2005 and was run for 48 hours, ending at 12 UTC 15 Jan 2005. The Eta $218^{3}$ analysis and forecasts from 12 UTC 13 Jan 2005 provided the initial and lateral boundary conditions respectively, and the boundary conditions were updated every 3 hours. The KF CP scheme was employed, and other parameterizations included the Ferrier microphysics scheme, RRTM longwave radiation scheme, Dudhia shortwave radiation scheme, the Noah land-surface model, and the YSU PBL scheme. Half-degree SST data were used.

\footnotetext{
${ }^{3}$ Eta forecasts interpolated to the World Meteorological Organization (WMO) 218 grid (approximately 12km grid spacing)
} 


\subsection{PV methodology}

An Ertel PV budget was performed for both the S1 and S2 case studies (Chapters 3 and 4), and a QGPV inversion was undertaken for the S2 case study (Chapter 4). The methodology for the budget and inversion very closely follow Lackmann (2002). As will be further discussed, PV is a useful framework within which assessing the impact of LHR on atmospheric dynamics is facilitated.

Extratropical cyclogenesis can be described from a PV standpoint as the mutual amplification of two or more preexisting, finite amplitude disturbances, a process which can be greatly enhanced by LHR (e.g., Davis and Emanuel 1991). In terms of PV, LHR enhances cyclonic development by both reducing static stability as well as by amplifying upper ridges downstream of the surface cyclones and the low-level PV maxima in the vicinity of cyclones. (The former allows for greater mutual interaction of the upper and lower disturbances by increasing the vertical penetration of the circulations.)

Ertel PV has two main advantageous properties; the first is that it is a conserved quantity for adiabatic, frictionless flow. By identifying areas in which PV is not conserved, this property allows for attribution to diabatic processes. The second is that it is invertible, and this property allows for the quantification of flow associated with specific PV anomalies. Combined, these properties demonstrate why PV is a useful framework in which to consider the impact of LHR on atmospheric dynamics. 


\subsubsection{Ertel PV budget}

Following Lackmann (2002), the PV budgets performed in the present study adopt the methodology set forth by Cammas et al. (1994), which is based largely on the work of Raymond (1992). The main purposes of performing the PV budgets are to provide a means of identifying lower-tropospheric PV anomalies that have been generated or enhanced by LHR, and to determine whether LHR is capable of producing a given PV feature.

The standard WRF model output does not include model latent heating fields, thus they are computed according to a parameterization formulation set forth by Emanuel et al. (1987):

$$
\frac{d \theta}{d t}=\omega\left(\frac{\partial \theta}{\partial p}-\frac{\gamma_{m}}{\gamma_{d}} \frac{\theta}{\theta_{e}} \frac{\partial \theta_{e}}{\partial p}\right)
$$

where $\gamma_{\mathrm{m}}$ and $\gamma_{\mathrm{d}}$ are the moist- and dry-adiabatic lapse rates, respectively, $\theta$ is the potential temperature, $\theta_{e}$ is the equivalent potential temperature, $p$ is the pressure, and $\omega$ is the isobaric vertical motion. This equation was applied in regions of ascent $(\omega<0)$ and saturation. Saturation was defined by relative humidity in excess of $70 \%$ when the temperature exceeded $263 \mathrm{~K}$, and when the temperature was below $263 \mathrm{~K}$, the relative humidity threshold was scaled by the departure of the environment from $263 \mathrm{~K}$. These threshold values were used in order to account for saturation with respect to ice in regions of subfreezing cloud, and also to allow for LHR in convective regions where gridboxes need not be saturated (adapted from Lackmann 2002).

The isobaric PV budget equation is given by: 


$$
\frac{\partial P}{\partial t}=-\nabla_{p} \bullet\left(P \vec{V}_{h}\right)-\frac{\partial}{\partial p}(P \omega)-g \nabla_{3} \bullet \vec{Y}
$$

where $\mathrm{P}$ is the Ertel $\mathrm{PV}$,

$$
P=g\left(f \hat{k}+\nabla_{3} \times V_{h}\right) \bullet \nabla_{3} \theta
$$

and $\overrightarrow{\mathbf{Y}}$ is the nonadvective PV flux vector,

$$
\vec{Y}=-\left(\frac{d \theta}{d t}\right)\left(f \hat{k}+\nabla_{3} \times V_{h}\right)+\nabla_{3} \theta \times \vec{F}
$$

In equations (2) - (4), $f$ is the Coriolis parameter, $g$ is the gravitational acceleration, $V_{h}$ is the horizontal wind vector, $\nabla_{p}$ is the quasi-horizontal gradient operator on an isobaric surface, $\nabla_{3}$ is the three-dimensional gradient operator $\left[\nabla_{p}-\hat{k}(\partial / \partial p)\right]$, and $\vec{F}$ is the frictional force vector. The frictional term $(\vec{F})$ is not considered here in order to focus on LHR associated with precipitation. The PV budget equation (Eq. 2) shows that the local time rate of change of Ertel PV is due to horizontal and vertical PV flux divergences, in addition to a nonadvective (diabatic) PV flux, which is distributed along the negative of the absolute vorticity vector. Therefore, when significant vertical wind shear exists, a considerable horizontal displacement will exist between negative PV tendencies aloft and positive PV tendencies below.

\subsubsection{Quasigeostrophic potential vorticity inversion}

A potential vorticity inversion was also performed for S2 (Chapter 4), using the quasigeostropic form of the PV (QGPV). Despite the fact that its underlying assumptions are more restrictive relative to Ertel PV, QGPV offers the advantage of linearity, making its inversion simpler and its results less ambiguous (see Davis 1992b for a comparison of 
QGPV and Ertel PV inversion). The disadvantage of QGPV is the loss of accuracy when ageostrophy becomes important, but the relatively straight nature of the feature examined in S2 indicates that the QGPV approach is likely acceptable, though this will be further defended in Chapter 4.

The QGPV inversion methodology used here is based on that of Hakim et al. (1996) and similar to that used by Lackmann et al. (1998) and Lackmann and Gyakum (1999). The QGPV is given by:

$$
q=\frac{1}{f_{0}} \nabla_{p}^{2} \phi^{\prime}+f+f_{0} \frac{\partial}{\partial p}\left(\frac{1}{\sigma_{r}} \frac{\partial \phi^{\prime}}{\partial p}\right),
$$

where $\phi^{\prime}$ is the deviation of the geopotential from the horizontally uniform International Civil Aeronautical Organization Standard Atmosphere, and $\sigma_{\mathrm{r}}=(\alpha / \theta)(\mathrm{d} \Theta / \mathrm{dp})$, a function of pressure only. It is useful to consider the QGPV as a sum of an arbitrary number of partitions such that $q_{*}=q-f=\sum_{i=1}^{n} q_{* i}$ where $n$ is the total number of subdivisions to the QGPV field, enumerated by the subscript $i$. Each $q_{* i}$ has a perturbation geopotential field $\phi_{i}^{\prime}$ associated with it, obtained through the inversion $\phi_{i}^{\prime}=\ell^{-1}\left(q_{*_{i}}\right)$, where $\ell=\left(1 / f_{0}\right) \nabla_{p}^{2}+f_{0}(\partial / \partial p)\left[\left(1 / \sigma_{r}\right)(\partial / \partial p)\right]$. Therefore, the corresponding geostrophic flow $\left(\vec{V}_{g}\right)$ is given by

$$
\vec{V}_{g}=\sum_{i=1}^{n} \vec{V}_{g i}=\sum_{i=1}^{n} \frac{1}{f_{0}} \hat{k} \times \nabla_{p} \phi_{i}^{\prime}
$$

For the QGPV inversion performed in Chapter 4, the QGPV was computed from WRF forecast fields in which the noisy height fields were smoothed by using a 9-point smoother. The QGPV was then partitioned into two parts: cyclonic low-level QGPV and 
anticyclonic low-level QGPV. That is, the cyclonic (anticyclonic) QGPV was made up of all negative (positive) QGPV below $600 \mathrm{hPa}$. The lateral boundaries were treated by letting all negative (positive) $\phi^{\prime}$ anomalies be counted in the cyclonic (anticyclonic) QGPV partition. Imposing a homogenous Dirichlet boundary condition (zero value imposed on the boundary) artificially reduces the magnitude of the solution in the loweratmosphere, if applied to a lower boundary of $1000 \mathrm{mb}$. To work around this complication, a lower boundary value of $1500 \mathrm{mb}$ was instead used, thereby allowing the solution to extend realistically to surface. The background PV field from which the QGPV and height perturbations were defined was determined by using 10-year European Center for Medium-Range Weather Forecasting (ECMWF) model climatological mean values.

The QGPV inversion was performed on the 20-km WRF model run described above. The 12-hour forecast time (F12) was used, based on strength and location of the system at that time. Results are discussed in Chapter 4. 


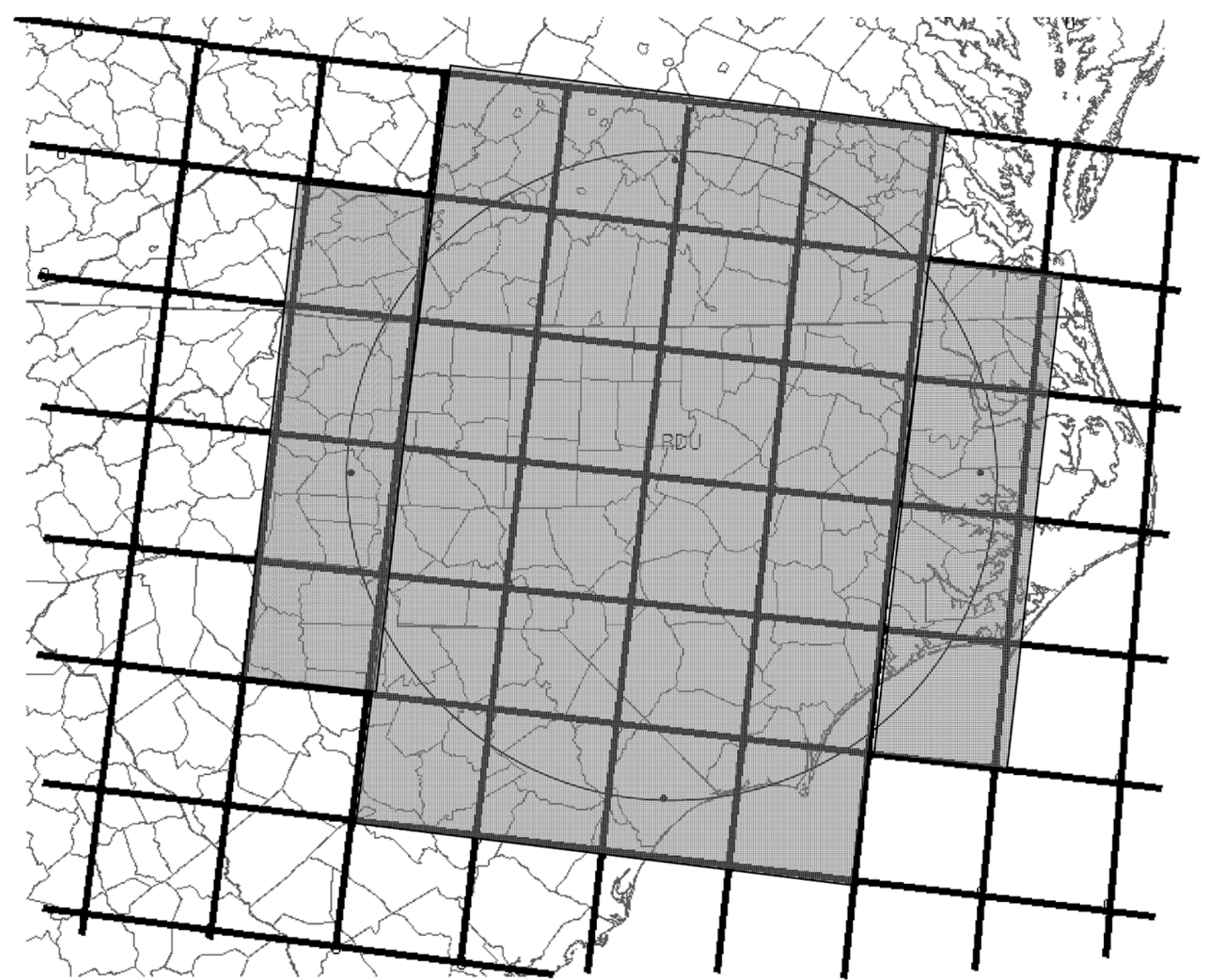

Figure 2.1. Area over which Eta forecasts and NARR precipitation analyses were averaged for the Eta QPF error climatology. Gray shading denotes the gridcells contained by the $200-\mathrm{km}$ radius from RDU. 


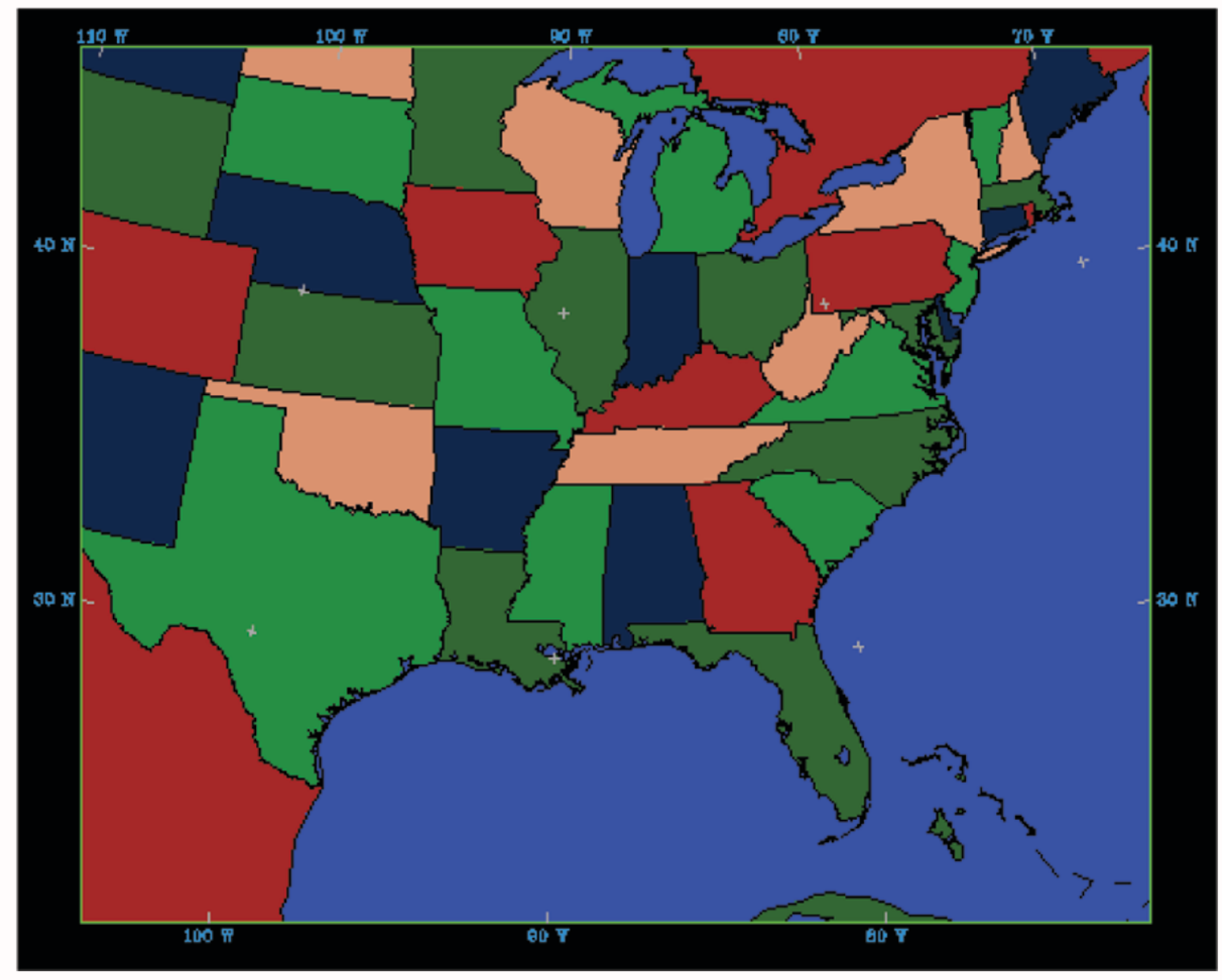

Figure 2.2. Model domain used in S1 MM5 simulation. 


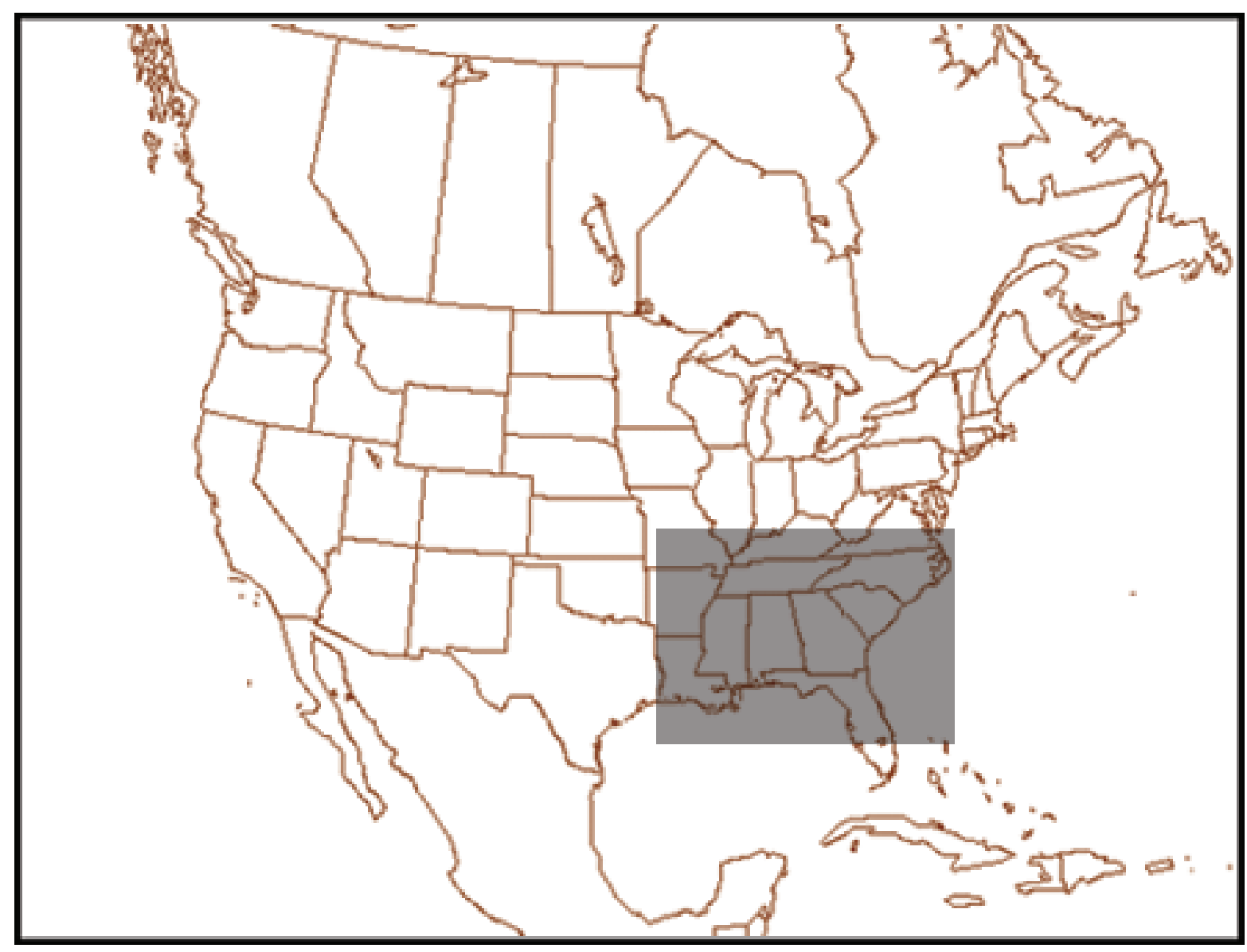

Figure 2.3 Model domain used in S1 WRF4 forecast. 


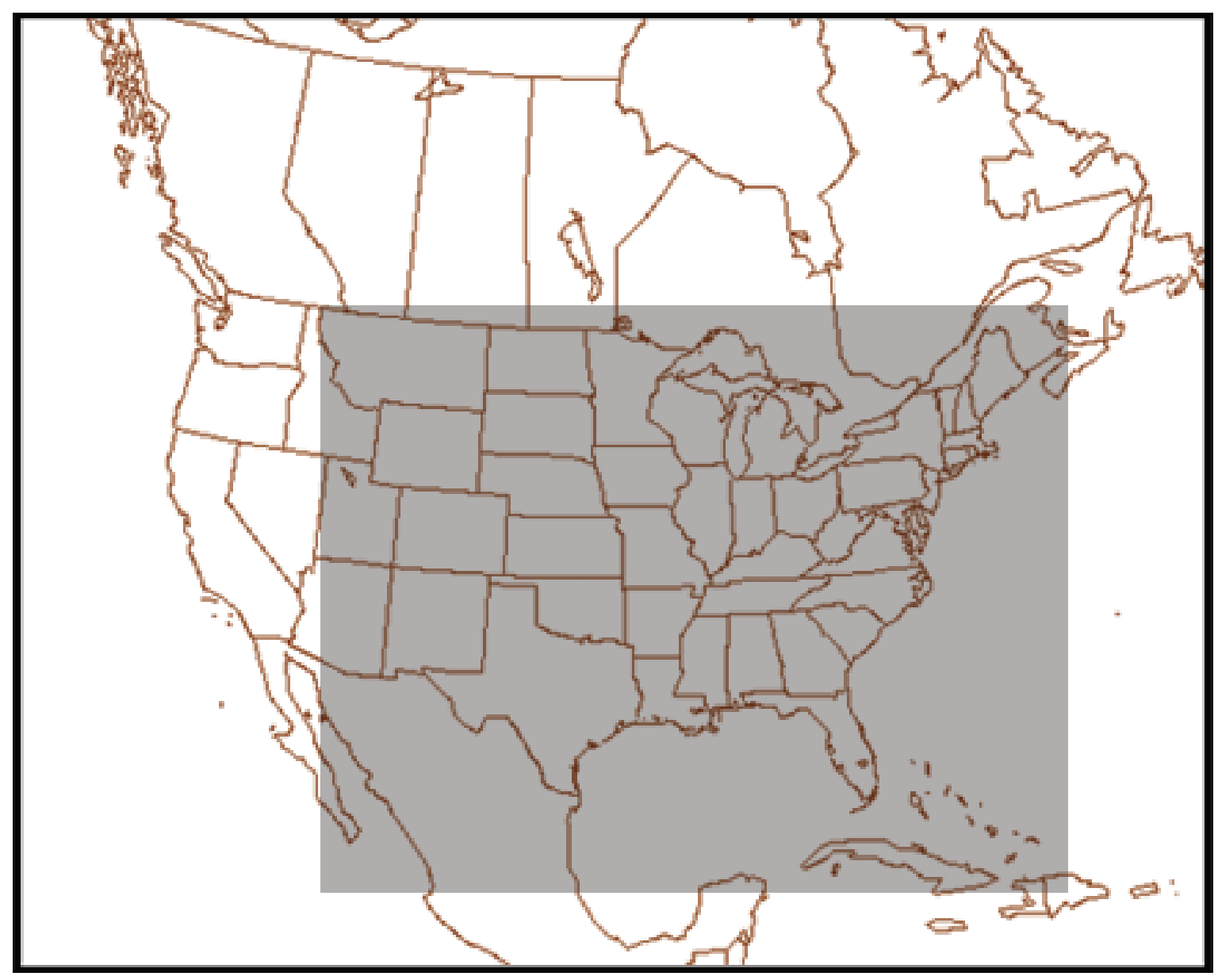

Figure 2.4 Model domain used in S1 WRF20 forecast. 


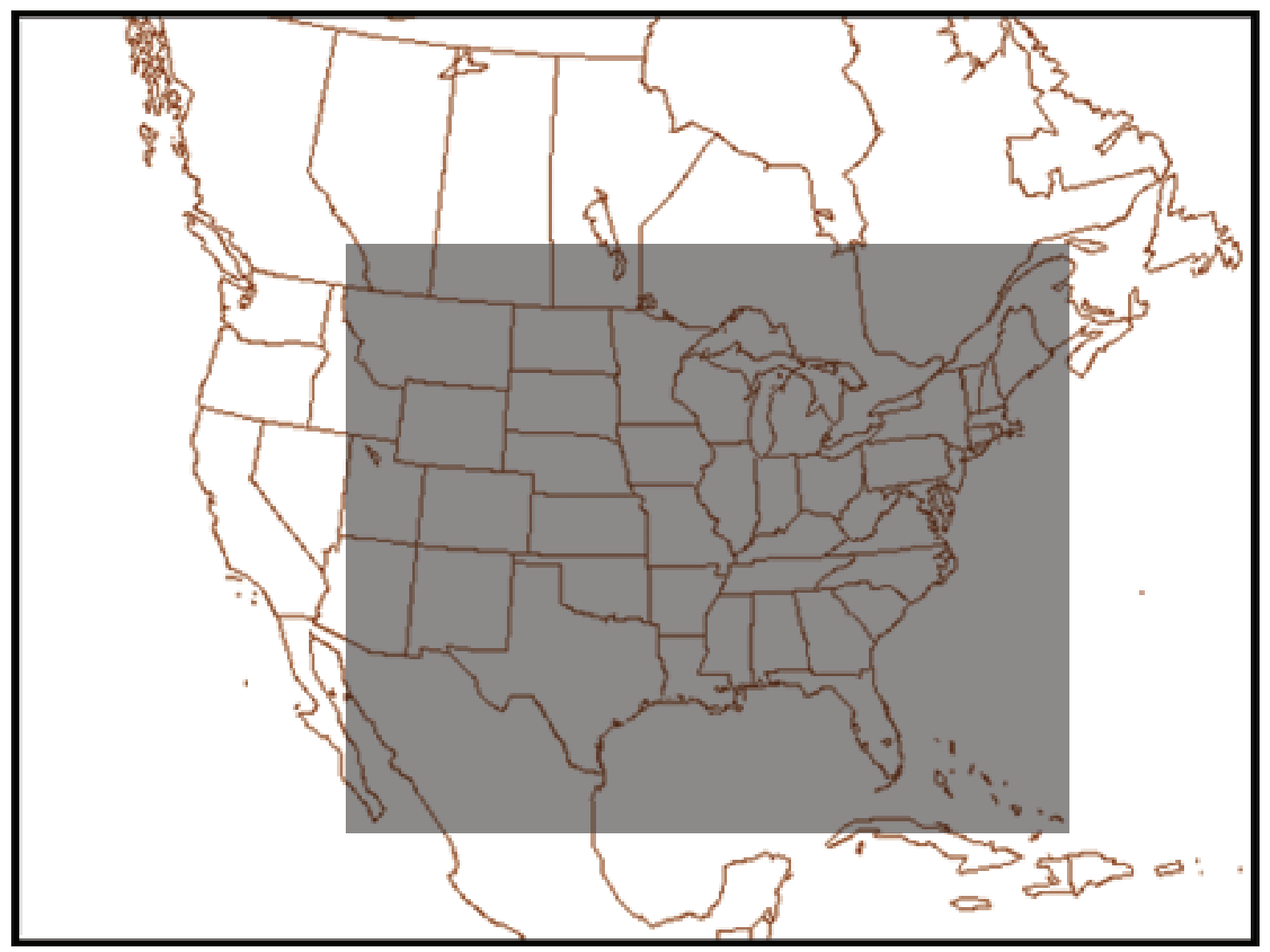

Figure 2.5 Model domain used in S2 WRF forecast. 


\begin{tabular}{|c|c|c|c|c|c|c|c|c|}
\hline DATEltime & Met threshold? & QPF & Prec Amt & Difference (Pred - Obs) & Over or Under pred? & Abs Val of Diff & Hit, Miss, FA? & Big Miss or False Alarm? \\
\hline 00010112 & no & $2.38 \mathrm{E}-02 \mathrm{no}$ & $-1.49 \mathrm{E}-02$ & 0.03866 & Over & 0.0386643 & Hit & \\
\hline 00010212 & no & 0.202381 no & $-1.70 \mathrm{E}-02$ & 0.21939 & Over & 0.2193889 & Hit & \\
\hline 00010312 & no & $7.14 \mathrm{E}-02 \mathrm{no}$ & $6.25 \mathrm{E}-03$ & 0.06518 & Over & 0.06518352 & Hit & \\
\hline 00010412 & no & $3.2381 \mathrm{no}$ & 10.01634 & -6.77824 & Under & 6.77824 & Miss & \\
\hline 00010512 & no & 0 no & 0.437716 & -0.43772 & Under & 0.437716 & Hit & \\
\hline 00010612 & no & 5.63095 no & 0.47604 & 5.15491 & Over & 5.15491 & False Alarm & \\
\hline 00010712 & no & 0 no & 0.687901 & -0.68790 & Under & 0.687901 & Hit & \\
\hline 00010812 & no & 2.07143 no & $5.23 \mathrm{E}-02$ & 2.01911 & Over & 2.0191096 & Hit & \\
\hline 00010912 & no & 11.7857 no & 10.7721 & 1.01360 & Over & 1.0136 & Hit & \\
\hline 00011012 & no & 0 yes & 18.6694 & -18.66940 & Under & 18.6694 & Miss & Big Miss \\
\hline 00011112 & no & 0 no & $3.40 \mathrm{E}-04$ & -0.00034 & Under & 0.000339569 & Hit & \\
\hline 00011212 & no & 0 no & $-9.69 \mathrm{E}-03$ & 0.00969 & Over & 0.0096875 & Hit & \\
\hline 00011312 & no & 0 no & $5.96 \mathrm{E}-02$ & -0.05964 & Under & 0.0596408 & Hit & \\
\hline 00011412 & no & 0 no & $-1.88 \mathrm{E}-03$ & 0.00188 & Over & 0.001875 & Hit & \\
\hline 00011512 & no & 0 no & 1.57E-02 & -0.01572 & Under & 0.0157185 & Hit & \\
\hline 00011612 & no & $1.19 \mathrm{E}-02 \mathrm{no}$ & $-6.86 \mathrm{E}-03$ & 0.01876 & Over & 0.01876256 & Hit & \\
\hline 00011712 & no & 3.67857 no & $-6.49 \mathrm{E}-03$ & 3.68506 & Over & 3.68505867 & Hit & \\
\hline 00011812 & no & 0.142857 no & 3.06352 & -2.92066 & Under & 2.920663 & Hit & \\
\hline 00011912 & yes & 26.7976 no & 1.67 & 25.12760 & Over & 25.1276 & False Alarm & Big False Alarm \\
\hline 00012012 & no & 0 no & 5.78085 & -5.78085 & Under & 5.78085 & Miss & \\
\hline 00012112 & no & 0 no & $6.49 \mathrm{E}-03$ & -0.00649 & Under & 0.00649115 & Hit & \\
\hline 00012212 & yes & 24.8095 no & 2.02316 & 22.78634 & Over & 22.78634 & False Alarm & Big False Alarm \\
\hline 00012312 & no & $1.27381 \mathrm{no}$ & 10.8258 & -9.55199 & Under & 9.55199 & Miss & \\
\hline 00012412 & yes & 18.5714 yes & 15.4329 & 3.13850 & Over & 3.1385 & Hit & \\
\hline 00012512 & no & 0.154762 yes & 18.9362 & -18.78144 & Under & 18.781438 & Miss & Big Miss \\
\hline 00012612 & 999 & no & $9.61 \mathrm{E}-02$ & -0.09612 & Under & 0.0961197 & Hit & \\
\hline 00012712 & 999 & no & $-1.70 \mathrm{E}-02$ & 0.01701 & Over & 0.0170079 & Hit & \\
\hline 00012812 & 999 & no & $-1.87 \mathrm{E}-03$ & 0.00187 & Over & 0.00187499 & Hit & \\
\hline 00012912 & yes & 17.6548 no & 1.09698 & 16.55782 & Over & 16.55782 & False Alarm & \\
\hline 00013012 & no & 1.14286 yes & 19.7156 & -18.57274 & Under & 18.57274 & Miss & Big Miss \\
\hline 00013112 & 999 & no & 0.665606 & -0.66561 & Under & 0.665606 & Hit & \\
\hline 00020112 & no & 0 no & $8.87 \mathrm{E}-02$ & -0.08868 & Under & 0.0886763 & Hit & \\
\hline 00020212 & no & 0 no & $4.01 \mathrm{E}-04$ & -0.00040 & Under & 0.000401074 & Hit & \\
\hline 00020312 & no & $3.57 \mathrm{E}-02 \mathrm{no}$ & 0.109469 & -0.07375 & Under & 0.0737547 & Hit & \\
\hline 00020412 & no & $1.19 \mathrm{E}-02 \mathrm{no}$ & $9.70 \mathrm{E}-02$ & -0.08508 & Under & 0.0850761 & Hit & \\
\hline 00020512 & no & 0 no & $1.88 \mathrm{E}-03$ & -0.00188 & Under & 0.00187746 & Hit & \\
\hline 00020612 & no & 0 no & $9.14 \mathrm{E}-03$ & -0.00914 & Under & 0.00913632 & Hit & \\
\hline 00020712 & no & 0 no & 3.17E-03 & -0.00317 & Under & 0.00316931 & Hit & \\
\hline 00020812 & no & 0.642857 no & $1.59 \mathrm{E}-02$ & 0.62695 & Over & 0.6269539 & Hit & \\
\hline 00020912 & no & 0 no & $6.44 \mathrm{E}-02$ & -0.06438 & Under & 0.0643775 & Hit & \\
\hline 00021012 & no & 0 no & $-6.49 \mathrm{E}-03$ & 0.00649 & Over & 0.00648867 & Hit & \\
\hline 00021112 & no & 2.77381 no & $5.47 \mathrm{E}-02$ & 2.71909 & Over & 2.7190905 & Hit & \\
\hline 00021212 & no & 1.05952 yes & 18.0265 & -16.96698 & Under & 16.96698 & Miss & \\
\hline 00021312 & yes & 22.5 no & 1.00686 & 21.49314 & Over & 21.49314 & False Alarm & Big False Alarm \\
\hline 00021412 & no & $8.33 \mathrm{E}-02 \mathrm{no}$ & 11.2786 & -11.19527 & Under & 11.1952667 & Miss & \\
\hline 00021512 & no & 0 no & $-4.40 \mathrm{E}-03$ & 0.00440 & Over & 0.00439714 & Hit & \\
\hline 00021612 & no & 0 no & $-3.37 \mathrm{E}-04$ & 0.00034 & Over & 0.000337105 & Hit & \\
\hline 00021712 & no & 7.21429 no & $5.21 \mathrm{E}-02$ & 7.16222 & Over & 7.1622156 & False Alarm & \\
\hline
\end{tabular}

Table 2.1 Sample selection of the Eta QPF error climatology 


\section{S1 Case Study: 31 Dec 2002 - 01 Jan 2003}

\subsection{Introduction/event summary}

The case chosen to represent Scenario 1 (S1) occurred on 31 Dec 2002, and featured a squall line moving eastward along the coast of the northern Gulf of Mexico that, despite few severe weather reports, produced significant rainfall and damaging winds (Fig. 3.1). The MCS originated in a trough of low pressure associated with a surface cold front, but as the convective system became better-organized it began to race out ahead of the front, moving quickly eastward through the Gulf states (Fig. 3.2).

Operational model precipitation forecasts (particularly those of the Eta model) over-forecasted the downstream QPF for this event, and a major forecast false alarm occurred across western and central SC/NC/VA, with QPF errors in excess of $35 \mathrm{~mm}$ present across western and central SC (Fig. 3.3). The purpose of this analysis is to investigate how the upstream MCS may have impacted downstream precipitation, and why NWP models were unable to adequately forecast this event.

\subsection{Event analysis}

\subsubsection{Observational analysis}

\subsubsection{Synoptic scale analysis}

At 12 UTC on 31 Dec 2002, radar imagery reveals that a region of UC is evident in the form of a potent MCS moving across eastern LA and MS (Fig. 3.2a). At the 250-, 500-, and 850-hPa levels, a closed low is apparent, along with a closed low and an associated trough of low pressure at the surface (Fig. 3.4). Winds at $250 \mathrm{hPa}$ are greater 
than $75 \mathrm{kts}$ and generally southwesterly across the Southeast US. A 500-hPa absolute vorticity maximum in excess of $3.2 \times 10^{-4} \mathrm{~s}^{-1}$ is present in the base of the trough at 500 $\mathrm{hPa}$, and mixing ratio values exceeding $10 \mathrm{~g} / \mathrm{kg}$ also precede the system at the $850-\mathrm{hPa}$ level. The southerly winds at low levels and the strong westerly component of the flow at upper levels produce a veering profile across the region.

By 00 UTC 1 Jan, the system has moved eastward at all levels (Fig. 3.5), and the northern branch of the jet stream has increased in strength (Fig. 3.5a). It can be seen at the $500-\mathrm{hPa}$ and $850-\mathrm{hPa}$ levels that the system has also deepened, and the tongue of moist air evident at 12 UTC 31 Dec has weakened across the GA region. The MCS (cf. Figs. 3.2e, 3.5d) is also further ahead of the surface trough than it was 12 hours earlier.

Most of the MCS has moved off of the East Coast by 12 UTC 1 Jan, with the northern end moving over eastern NC (Fig. 3.2h). At all levels the system has begun to weaken slightly, though strong low-level southerly flow has maintained Atlantic moisture across eastern NC and VA (Fig. 3.6).

\subsubsection{Mesoscale analysis}

Figures 3.7 - 3.9 show the mesoscale environment from 12 UTC 31 Dec to 12 UTC 1 Jan. At 12 UTC 31 Dec, lapse rates show that that the regions of largest instability are found over the Gulf of Mexico and the Gulf Stream (Fig. 3.7a). Low-level shear is concentrated in the region along and just ahead of the MCS, and low-level PV is aligned with the MCS, as well as in the stable region behind the surface cold front (Fig. 3.7a,b). A moderately strong LLJ in excess of $45 \mathrm{kts}$ is present, mostly along and to the south of the convective system, weakening over the area in which the MCS is bowing most 
prominently (cf. Figs. 3.7c, 3.2a). The $850-\mathrm{hPa}$ moisture flux field also shows a maximum of moisture flux to the south of the system, with a region of weaker values to the along and immediately north of the MCS (Fig. 3.7d). Soundings taken in the preconvective environment at this time also depict a marked layer of mid-level dry air and a veering profile with westerly winds increasing with height (Fig. 3.8).

At 00 UTC 1 Jan, low-level shear and instability have increased along and ahead of the UC feature, and the low-level PV maxima have moved eastward with the system, the largest of which revealing the large region of post-convective stabilization in the wake of the MCS (Figs. 3.9a,b). The LLJ has weakened considerably, and is now located completely south of the MCS, in an amorphous and diluted configuration (Fig. 3.9c). Likewise, the moisture flux field reveals that the moisture flux maximum is well to the south of the convective system and decreases toward the north (Fig. 3.9d).

By 12 UTC 1 Jan (radar in Fig. 3.2i), convective instability is present over eastern $\mathrm{SC}, \mathrm{NC}$, and VA, and the low-level PV maxima continue to move eastward with the system (Fig 3.10a,b). The LLJ is maximum ahead of the system, which is now located over the Atlantic ocean, though sufficient southerly flow persists over land such that the moisture flux values increase over eastern SC, NC, and VA (Figs. 3.10c,d).

\subsubsection{Analysis of operational forecast}

The operational Eta model forecast initialized at 00 UTC 31 Dec produced a large QPF error over portions of western $\mathrm{NC} / \mathrm{SC}$ and northern GA, where observed precipitation totals were up to $35 \mathrm{~mm}$ less than those forecasted by the operational Eta model (Fig. 3.3). In order to determine how well the operational model forecast verified 
overall, the Eta forecast is compared to observations and analyses in the following sections.

\subsubsection{Assessment of Eta QPF}

Figure 3.11 compares the observed precipitation totals over the course of the event to the evolution of the system as it was represented in several model forecasts. In this section, only the first two rows will be discussed; the other forecasts will be described in the sections that follow.

Comparing row A (the NARR precipitation analysis) to row B (the operational Eta QPF) of Fig. 3.11 reveals the magnitude of the Eta model QPF error for this case. The Eta model not only predicted too much precipitation, but the forecast speed of the precipitation system movement was significantly too slow, as the analyzed precipitation is well offshore (and thus not captured by the NARR reanalysis) while the Eta model is still generating large amounts of precipitation over the Southeast. Additionally, one can note similar errors by comparing the Eta model QPF sequence in Fig. 3.11 to the radar sequence shown in Fig. 3.2.

A comparison of the Eta model 24-h QPF ending at 00 UTC 01 Jan to the corresponding NARR 24-h precipitation analysis (Fig. 3.3) reveals a large Eta model overprediction of precipitation over western $\mathrm{NC} / \mathrm{SC}$ and northeastern GA. This is quantified in the difference plot shown in Fig. 3.12, revealing that the Eta model's QPF

error was most pronounced over portions of $\mathrm{NC}, \mathrm{SC}$ and $\mathrm{GA}$, as the model forecast overpredicted the 24-h precipitation total in those areas by more than $35 \mathrm{~mm}$. 
The error is elucidated further by examining precipitation totals during the most critical 6-h interval (Fig. 3.13). As Fig. 3.13 shows, the erroneous Eta model forecast moved the convective system north and east over the Carolinas and GA, while observations at this time showed that system actually had moved due eastward and was located offshore, east of FL and GA (Fig. 3.2). The observed precipitation totals are not well-depicted in Fig. 3.13, as the NARR precipitation analysis does not reliably extend offshore, but the comparison to the operational model forecast for inland regions clearly shows the Eta model error.

\subsubsection{Eta synoptic scale forecast analysis}

The 12-hour forecast (F12) valid at 12 UTC 31 Dec reveals that many of the main synoptic features apparent in the analyses are well-represented in the forecast (cf. Figs. 3.4, 3.14). At $250 \mathrm{hPa}$, a cutoff low and southwesterly flow are common to both the forecast and the analysis, and at $500 \mathrm{hPa}$, the height and vorticity fields are also similar, though the cutoff low is approximately $60 \mathrm{~m}$ deeper in the analysis than in the forecast (cf. Figs. 3.4a,b, 3.14a,b). The forecast and analysis environments at $850 \mathrm{hPa}$ and the surface are in fairly good agreement (cf. Figs 3.4c,d, 3.14c,d).

Comparing the 24-hour forecast valid at 00 UTC 1 Jan to the analysis shows that the forecasted system is slightly too weak at upper levels; 250-hPa winds are approximately $25 \mathrm{kt}$ too weak in the area preceding the MCS, and the 500-hPa closed low remains approximately $60 \mathrm{~m}$ too weak in the forecast (cf. Figs. 3.5a,b, 3.15,a,b). At 850 $\mathrm{hPa}$, the model forecast of mixing ratio and height are both quite poor; the model forecast overestimates the central height by approximately $30 \mathrm{~m}$, and while analyses show a clear 
minimum in the mixing ratio field, the model forecast spreads mixing ratio values continuously northward (cf. Figs. 3.5c, 3.15c), along the area over which the model precipitation is generated (Fig. 3.15d).

By 12 UTC 1 Jan, the model forecast at upper levels is in close agreement with analyses, while at lower-levels there is disagreement between the analyzed and forecasted moisture and precipitation fields. Figure 3.16 reveals that only slight errors are evident in the model forecast at upper levels, as $250-$ and $500-\mathrm{hPa}$ heights, winds, and vorticity values are in good agreement with those analyzed (cf. Figs. 3.6a,b, 3.16a,b). At $850 \mathrm{hPa}$ the mixing ratio values are larger and displaced to the west of those seen in the analysis at this time (cf. Figs. 3.6c, 3.16c). As seen by both the 850 -hPa mixing ratio values and the surface temperature field, it is clear that the convective system in the model forecast moved too slowly eastward relative to what was observed (cf. Figs. 3.6c,d, 3.16c,d). Figure 3.16 shows that overall, the mid- and upper levels of the atmosphere are well represented by the model forecast, while the low-levels reveal larger errors. This finding reveals that the major model challenge is likely convective propagation, not synoptic system evolution.

\subsubsection{Eta mesoscale forecast analysis}

Mesoscale forecast fields for the operational Eta forecast valid at 12 UTC 31 Dec show that low-level shear and convective instability are fairly well-represented, while the low-level PV field at this time already slightly lags toward the west relative to the analysis (cf. Figs. 3.7a,b, 3.17a,b). The forecasted LLJ is approximately $10 \mathrm{kt}$ too weak in the region preceding the MCS, and the $850-\mathrm{hPa}$ moisture flux is likewise slightly too 
weak over the same area, as well as to the north, over western TN (cf. Figs. 3.7c,d, $3.17 \mathrm{c}, \mathrm{d})$.

By 00 UTC 1 Jan, the model forecast does not represent the area of enhanced convective instability ahead of the MCS evident in the analysis, and the low-level PV field shows a significantly different configuration relative to that analyzed (cf. Figs 3.9a,b, 3.18a,b). The most significant difference at this time however, can be seen by comparing Figs. 3.9c,d and 3.18c,d. The strength and configuration of the LLJ and the associated moisture flux fields are distinctly different between the forecast and the analysis. The model forecast produces a LLJ axis ahead of the forecasted MCS, allowing excessive moisture to be passed into the downstream area via the enhanced moisture flux that is too large relative to the analysis. This is an important difference, and suggests that the physical mechanisms of moisture consumption and LLJ interruption ((i) and (iii) from section 1.2.1) may play a significant role for this event.

The 36-hour forecast valid at 12 UTC 1 Jan shows that the convective instability and low-level PV continue to be located too far toward the west relative to the analysis fields. An important difference is still seen in the low-level wind and moisture flux plots (cf. Figs. 3.10, 3.19). The comparison of the forecast and analyzed moisture flux fields confirms that excessive moisture in the forecasted downstream area is consistent with the overprediction of the downstream QPF over western and central SC, NC, and VA.

The synoptic and mesoscale forecast evaluations suggest that the forecast error produced by the operational Eta model was two-fold: First, the speed at which the forecasted MCS moved was too slow; and second, the QPF within the system itself was too low along the southern (upstream) end of the system and too high in the downstream 
region. These errors also appear to be manifested in the similarly erroneous low-level wind and moisture flux fields.

\subsection{Numerical simulations}

In order to better understand the physical processes that occurred in the MCS and its surrounding environment and how they differed from those predicted by operational forecasts, several model runs of this event were performed. The model output is used both as a surrogate observational dataset, as well as for diagnostic investigations (e.g., Keyser and Uccellini 1987).

First, MM5 was used to attempt to produce a better downstream QPF, yet despite trying numerous configurations, most forecasts bore an unacceptable resemblance to the erroneous operational Eta forecast. The MM5 run that used the KF CP scheme and is described in section 2.2.2 is shown in Fig. 3.11, row C. While the forecast appears to be a relative improvement over the operational Eta forecast (though some of the difference may be due to the higher resolution used in the MM5 run), the model forecasted movement of the upstream MCS remains too slow, and the downstream QPF too high. Similar errors were reflected in all of the other MM5 runs that were performed using various $\mathrm{CP}$ schemes and resolutions.

WRF model forecasts were also produced; one using 20-km gridspacing and the KF CP scheme (WRF20, hereafter), the other using 4-km gridspacing and no CP scheme (WRF4, hereafter). The QPF fields that each of these model runs produced are shown in

Fig. 3.11, rows D and E, respectively. As Fig 3.11 shows, the WRF4 run yielded the superior QPF, though the WRF20 run did reveal large improvements relative to the 
operational Eta forecast despite similar grid-spacing to the operational Eta model and the use of a CP scheme. This improvement suggests that an inherent difference between the WRF model and the Eta and MM5 models exists, but examining this is beyond the scope of the current study. The WRF20 run will be utilized in the PV budget discussed later in this chapter. Because WRF4 produced the superior forecast, it was selected as the run with which to further examine this case. A detailed description of the WRF4 run is given in section 2.2.2. The forecast produced by this model run and a comparison to the operational Eta model forecast are discussed below.

\subsubsection{WRF4}

\subsubsection{Assessment of WRF4 QPF}

The QPF produced by the WRF4 run is shown in row E of Fig. 3.11. Relative to the other three model runs shown, both the speed of the convective system as well as localized precipitation amounts were better represented when compared to radar observations (Fig. 3.2), and the NARR analysis (Fig. 3.11, row A). While the southern end of the convective system in the WRF4 forecast is likely restricted by the small domain and the associated lateral boundary condition concerns, the speed of the system and the QPF in the downstream area are much improved relative the Eta forecast. Figure 3.20 shows that the downstream QPF generated by the WRF run over northeast GA, western SC, and $\mathrm{NC}$ is a significant improvement over that given by the operational Eta model forecast. The WRF4 24-h QPF shows a relative minimum over northeastern GA, and western NC and SC (where approximately less than $12 \mathrm{~mm}$ of precipitation fell), while the Eta forecast reveals a maximum (of over $55 \mathrm{~mm}$ ). Though the WRF4 24-h QPF 
is far from a perfect forecast, the degree of improvement, combined with the improvement in the forecast of the speed and intensity of the UC shows that the WRF4 forecast better represents the observed system. The possible reasons for this will be discussed in the following section.

\subsubsection{WRF4 mesoscale forecast analysis}

Because the domain of the WRF4 model run was limited in size due to computational considerations (Fig. 2.4), the synoptic forecast analysis is omitted here. In this section, four-panel mesoscale plots from the WRF4 run are shown and compared to Eta analyses and forecasts discussed in previous sections.

At 12 UTC 31 Dec, the WRF4 12-h forecast closely resembles the Eta forecast in many ways (aside from resolution-based differences), likely due to the influence of the Eta 211 initial conditions (cf. Figs. 3.17, 3.21). The 850-hPa LLJ is slightly too weak relative to analyses, as is the low-level moisture flux (cf. Figs. 3.7c,d, 3.21c,d).

By 00 UTC 1 Jan, the low-level PV fields show that the WRF system has moved further east at this time than was represented in the Eta forecast (cf. Figs. 3.18b, 3.22b). A significant feature of this forecast period, however, is the representation of the LLJ and 850-hPa moisture flux. Figure 3.23 shows a comparison of these fields as represented by the Eta analysis, the Eta forecast, and the WRF4 forecast. A comparison of panels (a), (c), and (e) show that the distinct north-south orientation of the LLJ in the Eta forecast does not match the weaker, less-defined configuration seen in the analysis and WRF4 forecast. The WRF4 LLJ forecast is significantly stronger than that seen in the analysis. Likewise, this same pattern appears in the moisture flux fields (Fig. 3.23b,d,f). In the WRF4 
forecast, the moisture flux maximum is suppressed along the southern end of the convective system (as in the analysis), whereas in the Eta forecast, the moisture flux maximum extends uninhibited into the downstream area.

The WRF4 36-h forecast valid at 12 UTC 1 Jan continues to trend more toward the analysis, as its low-level shear, wind, and moisture flux fields all bear more of a resemblance to the analysis than to the Eta forecast (cf. Figs. 3.10, 3.19, 3.24). The WRF4 forecast at this time places the axis of maximum moisture flux along the East Coast, extending into eastern $\mathrm{NC}$ (matching the analysis at this time), while the Eta forecast lags the maximum too far to the west. The reenergized moisture flux over eastern $\mathrm{NC}$ is consistent with the production of the precipitation that fell in that region toward the end of the event (Fig. 3.2g,h).

\subsubsection{Physical mechanisms of downstream precipitation alteration}

Comparing the operational Eta and WRF4 forecasts to analyses, it is apparent that there are large physical differences between the UC/downstream precipitation scenarios that develop in each. The most significant differences appear to be the speed at which the precipitation system moves and the relationship of the LLJ/moisture flux maxima to the regions of most intense precipitation.

Because the explicit convection in the WRF4 forecast moves more quickly (and in a more easterly direction) than does the precipitation generated by the operational Eta model, it is believed that the more slowly-moving Eta system allowed too much moisture to be transported ahead of the system (i.e. downstream). The resulting high moisture fluxes (as seen above) are consistent with the enhanced downstream model QPF, possibly 
explaining the positive QPF bias that resulted in the operational Eta model forecast. Conversely, the faster-moving convection in the WRF4 forecast allowed the axis of maximum moisture flux and LLJ to move eastward at a speed close to that which was observed. This adjustment seems to have allowed the LLJ and moisture flux maxima to be correctly placed south of the system, thus inhibiting excessive moisture flow to the north.

Given the comparisons that have been made between the model forecasts and analyses, it is possible to speculate as to which of the major physical mechanisms listed in Chapter 1 may be most significant to this case. As evidenced by comparable lapse rate and height fields in the forecast and analysis comparisons described above, neither downstream stabilization (mechanism (ii)) nor alternation of the synoptic dynamics (mechanism (iv)) appear to be of significant consequence to the downstream precipitation field for this event. However, the relative minimum of mixing ratio in Fig. 3.5, as well as the stunted northward extent of the LLJ and moisture flux maximum imply that the mechanisms of moisture consumption (i) and alteration of the LLJ (iii) may be important. Because the mixing ratio values in the downstream area do not differ greatly from those upstream, it is likely that the predominant process at work in this event is mechanism (iii) (the interruption of lower-tropospheric moisture transport), though the two mechanisms are clearly related.

\subsection{PV budget}

At the outset of this investigation it was hypothesized that the S1 UC systems would likely reveal a large propagative component to their motion, based on the speed of 
their observed motion. In order to investigate the validity of this hypothesis, as well as to clarify the role of system motion in the QPF bias for this case study, the PV framework was employed due the usefulness of PV as a traceable quantity that is not conserved for diabatic processes such as LHR (e.g. Davis and Emanuel 1991).

A low-level PV maximum accompanies an MCS due to the mid-level latent heating maximum that an MCS produces, and the quantity can therefore act as a useful tracer for the convective system. Many studies have examined MCSs from a PV perspective (e.g., Wang et al. 1993; Davis and Weismann 1994; Braun and Houze 1996). A PV budget was performed in order to examine the nature of the system movement; that is, to investigate whether the storm motion was largely advective, or whether propagation had a significant contribution, and what that may imply for the downstream precipitation field. The Ertel PV budget discussed below follows the methodology described in Chapter 2.

\subsubsection{WRF20 forecast evaluation}

While the WRF4 run discussed above provided the most accurate model run, a 20-km WRF model forecast was used for the PV investigation of this case for several reasons. First, excessive noise in WRF4 PV fields prevented a useful PV signal from being discernable. Second, this model run is coarse enough (and therefore requires the use of a $\mathrm{CP}$ scheme) that it captures the essence of model error of the system moving too slowly, while still retaining a fine enough resolution to resolve important mesoscale PV features. Finally, using the WRF20 output for this PV budget allowed for a better comparison to the corresponding PV budget performed for S2 in the following chapter. 
Compared to observations, the most significant aspect to note about this run is that the model-forecasted system moves eastward too slowly relative to observations (see Fig. 3.11). However, it was a vast improvement over the operational Eta forecast, especially as the system evolved in time. Therefore, this run is reliable enough to capture the UC system, while still exhibiting some of the model errors that were also associated with the operational forecast of this case. Ideally, the WRF4 run would have been used for this PV budget, but because of the excessive noisiness in the WRF4 PV fields, the results using the WRF20 run are discussed below.

\subsubsection{PV budget results}

Figure 3.25 shows the low-level PV and parameterized LHR from forecast hours 12,24 , and 36 . Because the squall line was most active (and in a location of significant forecast concern) at 12 UTC 31 Dec and 00 UTC 01 Jan, these times will be of particular focus. At all three forecast times, the squall line is represented by both a low-level PV maximum, as well as a maximum of latent heating (described in section 2.3.1). This collocation shows that the generation of the low-level PV maximum was very likely diabatic in nature.

Four-panel plots were generated to summarize the PV budgets at the forecast times F12 (12 UTC 31 Dec) and F24 (00 UTC 01 Jan) (Figures 3.26 and 3.27). In both figures it is evident that nonadvective (i.e., diabatic) PV tendencies (Figs. 3.26c, 3.27c) dominate relative to the horizontal and vertical advective fluxes (Figs. 3.26a,b, 3.27a,b, respectively). However, it is important to note that at F12 (Fig. 3.26), the PV tendencies in general are primarily collocated with the PV maxima, while at F24, positive tendencies 
(both advective and nonadvective) are located slightly to the east of ("ahead of") the system, as represented by the low-level PV (Fig. 3.27). At F12, this implies that little advection of the PV itself was actually taking place, while by F24, it appears that a combination of advective tendencies (primarily along the northern end of the line) (Fig. 3.27a) and nonadvective tendencies (primarily along the southern end of the line) (Fig. $3.27 \mathrm{c}$ ) acted to move the system toward the east.

Figures 3.28 and 3.29 show the latent heating and absolute vorticity vectors along cross-sections shown by the lines A-A' and B-B' printed in Fig. 3.25. At F12 (Fig. 3.28), a strong LHR and PV signature are evident, and are very closely collocated in the horizontal plane. By F24 (Fig. 3.29) both the LHR and PV have weakened considerably but remain collocated. Of particular note at this time is that the PV tower has begun to slope toward the east with height. This tilt may be due to the increasing westerlies with height, and it also supports the hypothesis that the surface winds may be too weak due to the omission of convective momentum transport by the $\mathrm{CP}$ scheme. (The coarse resolution of observations and analyses available makes this hypothesis a complicated one to test, and is instead left for future investigation.)

The section-normal wind speed along the same two cross-sections is shown with PV and absolute vorticity vectors in Figs. 3.30 and 3.31. A LLJ of about $25-30 \mathrm{kts}$ is evident in both of these figures, providing at least some of the moisture transport discussed in earlier sections. However, a cross-section taken to the north along $\mathrm{C}-\mathrm{C}^{\prime}$ shown in Fig. 3.32 shows that a much weaker southerly flow results, further supporting the idea that interruption of moisture transport via the interruption of the LLJ (mechanism (iii)) may contribute to reduced downstream precipitation in this case. 
Figure 3.33 shows the nonadvective PV flux tendencies along the cross-section lines A - A', B - B', and D - D', while Fig. 3.34 shows the corresponding advective PV flux tendencies associated with the system at F12, F24, as well as F36. At F12 (Figs 3.33a, 3.34a), both types of fluxes show positive PV tendencies below the system, but as noted above, little positive PV tendency is found east of (or "ahead" of) the system. This indicates that this WRF20 model run did not well represent the tendencies that likely moved the system forward at this time, possibly explaining its slow motion compared to observations. By F24 (panel b), a positive advective PV tendency can be seen immediately to the east of the PV tower, and the nonadvective PV flux tendency is also located toward the forward end of the system, slightly leading the PV maximum. At F36, the nonadvective PV tendencies no longer appear to aid the system's forward motion, as the positive PV tendencies are generally collocated with the PV tower, while the advective tendencies noticeably extend toward the leading edge of the system. The positive PV tendencies are consistent with the (negative of the) slope of the absolute vorticity vector, due to the increasing vertical shear in the vicinity of the convective system (cf. Figs. 3.7a, 3.9a, 3.10a). By this forecast time, it is clear that the forward motion of the convective system is at least somewhat more attributable to advective tendencies than it is to nonadvective tendencies.

In order to provide an independent check of the result that the S1 UC system moved largely via advection, a cursory calculation was performed. A rough estimate of average low-level wind speed was calculated by averaging the observed $850-\mathrm{hPa}$ wind speeds found along the UC system, and a rough estimate of system motion was calculated by tracing maximum radar echoes in time. Though the caluculations are inexact, it was 
found that the low-level wind speed average roughly matched the average speed at which the observed squall line moved (both quantities averaged to be approximately $11-13 \mathrm{~m} / \mathrm{s}$ over a 12-h period). This confirms that advection was likely the strongest contributor to forward motion for this case (though clearly not acting in isolation from propagative and nonadvective effects).

To extend this finding to the hypothesis discussed in the previous sections, it is important to point out that momentum transport as previously discussed is an advective process (that is, the downward transport of momentum accelerates the line in an advective sense). This may be the reason that the advective PV tendencies were more accountable for the convective motion later in the WRF20 simulation than earlier; that is, the model may be better equipped to handle the momentum transport as time progresses and the precipitation does not depend as heavily on the CP scheme. Furthermore, as discussed in section 1.3.3., explicit precipitation forecasts often lead to gridcell overturning, a process that transports momentum from upper-levels to the surface ${ }^{1}$. This idea presents many possibilities and will be explored further in the future.

As has been found in comparing the WRF4 explicit run to those that relied on a $\mathrm{CP}$ scheme, only the explicit run came close to representing a system movement speed close to that which was observed. Therefore, it is hypothesized that, were a suitable PV budget able to be performed on the 4-km output, advective tendencies may dominate even more due to the inclusion of the momentum adjustment process relative to their contribution seen here.

\footnotetext{
${ }^{1}$ However, momentum does not "mix" in the same sense as most other variables (e.g. Gallus and Johnson, Gao et al. 1990)
} 


\subsection{Summary}

The 31 Dec $2002-01$ Jan 2003 event is a S1 event in a large downstream model QPF error resulted when the operational Eta model was unable to accurately predict the movement of the UC system, as well as the associated LLJ and moisture fields. The operational Eta model forecasts showed a precipitation system that moved too slowly and generated too much precipitation downstream, resulting in a large model QPF false alarm in the northeastern $\mathrm{GA} /$ western and central $\mathrm{NC} / \mathrm{SC} / \mathrm{VA}$ region.

Accurate operational Eta forecasts of the upper-level low and surface front reveal that the operational model forecast erred the most with respect to convective movement, not the movement of the entire system. An experimental 4-km explicit WRF model run shows that when the $\mathrm{CP}$ scheme is shut off and the model is run at a resolution high enough to resolve convective motions (e.g. Weisman et al. 1997), a more accurate forecast of convective system movement results, and the downstream QPF improves as well.

It is hypothesized that $\mathrm{CP}$ schemes cannot presently realistically propagate convection due at least partially to the lack convective momentum adjustment from upper levels toward the surface. While CP schemes do contain hydrometeor, temperature, and moisture tendency fields, they are currently not designed to account for the transfer of momentum from upper to lower levels ${ }^{2}$ in the vicinity of a convective system. If this hypothesis is correct, then it is reasonable to suggest that only model forecasts employing explicit convection can realistically account for the impact that a fast-moving, low-level

\footnotetext{
${ }^{2}$ Though CP schemes do not account for the transport of momentum from low levels to upper levels either, the transfer of momentum from upper to lower levels is believed to be of greater importance with respect to convective system motion as discussed here.
} 
momentum-driven convective system might have on the surrounding environment, including the downstream precipitation field. This hypothesis will be the focus of future investigation. 


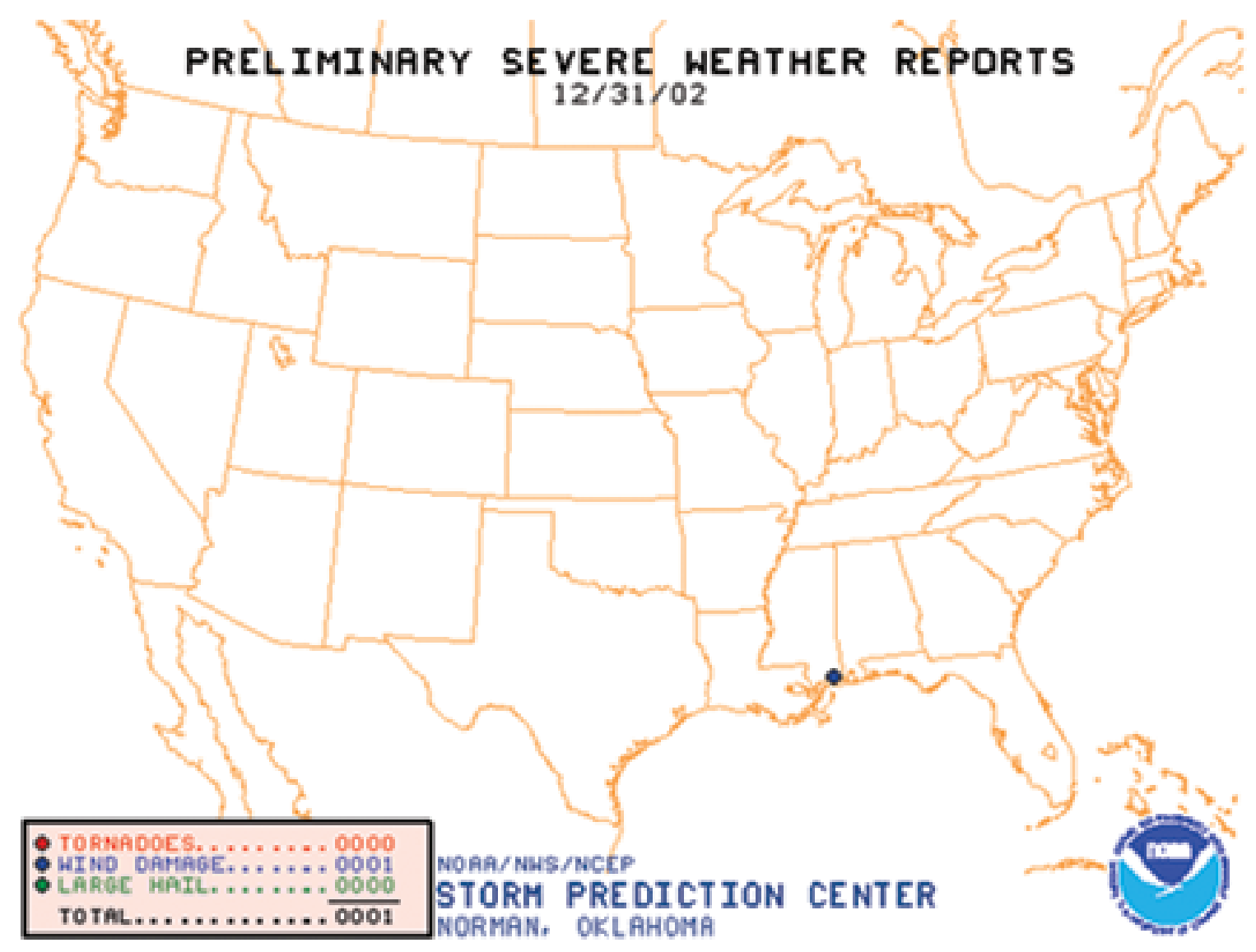

Figure 3.1. Severe weather reports as reported by the Storm Prediction Center for 31 Dec 2002. 


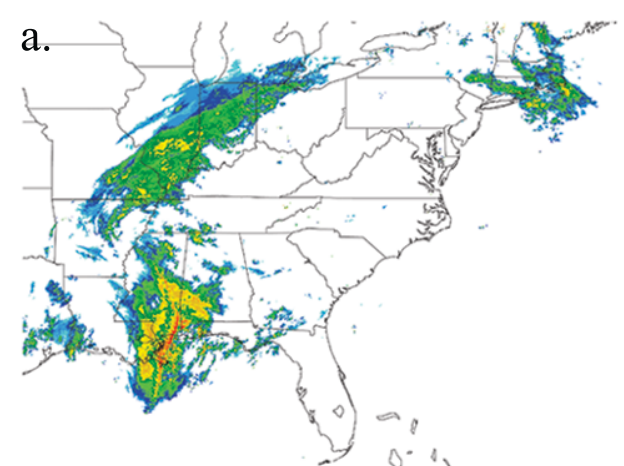

12 UTC $31 \mathrm{Pec}^{\circ}$.

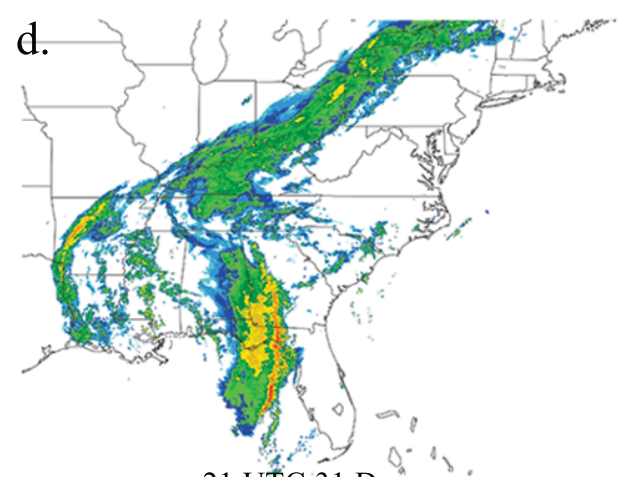

21 UTC 31 Dec

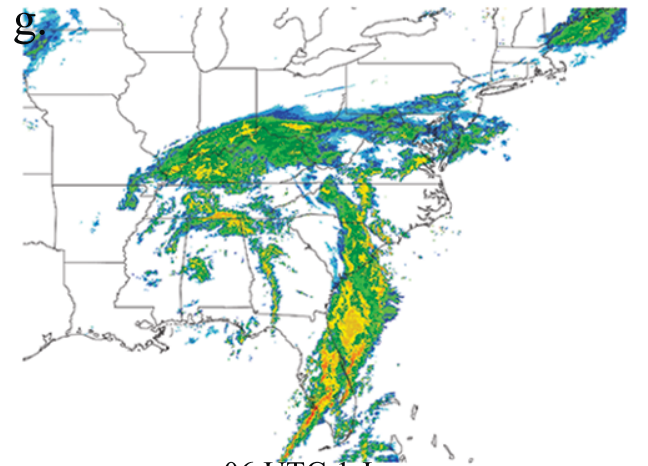

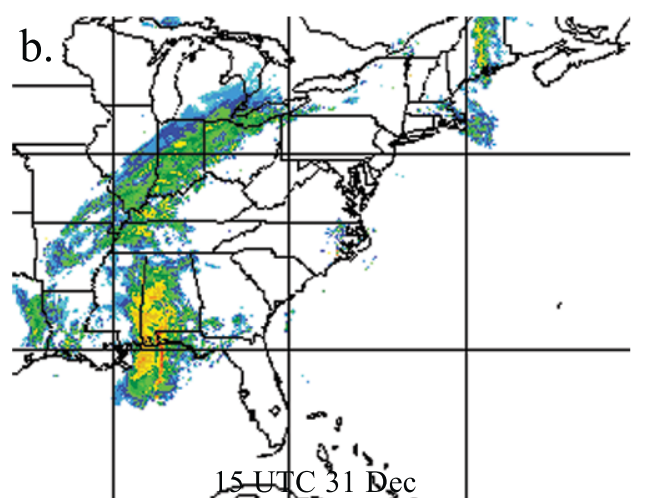

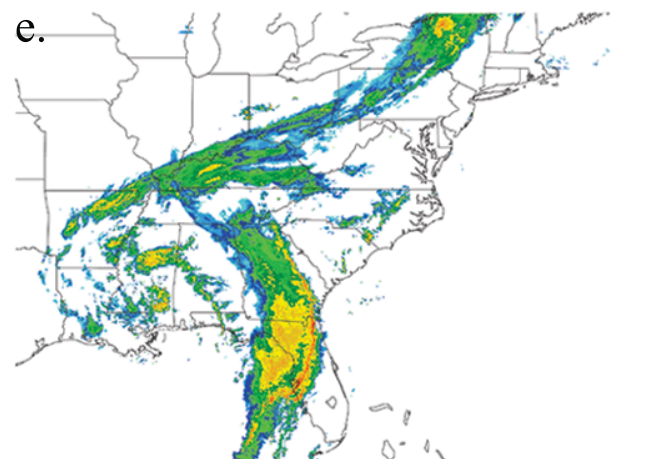

00UTC I Jan

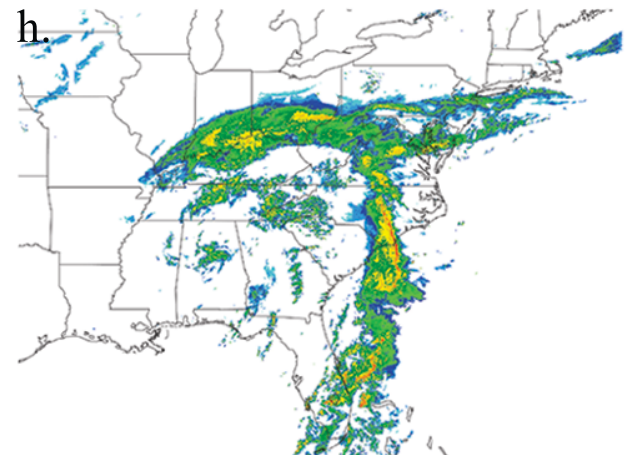

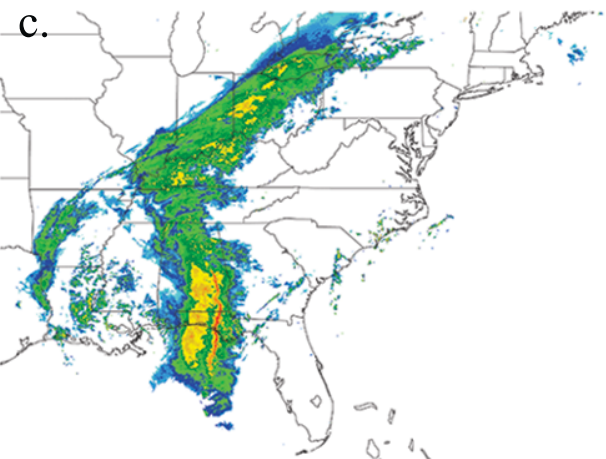

18 UTC 31 Dèc
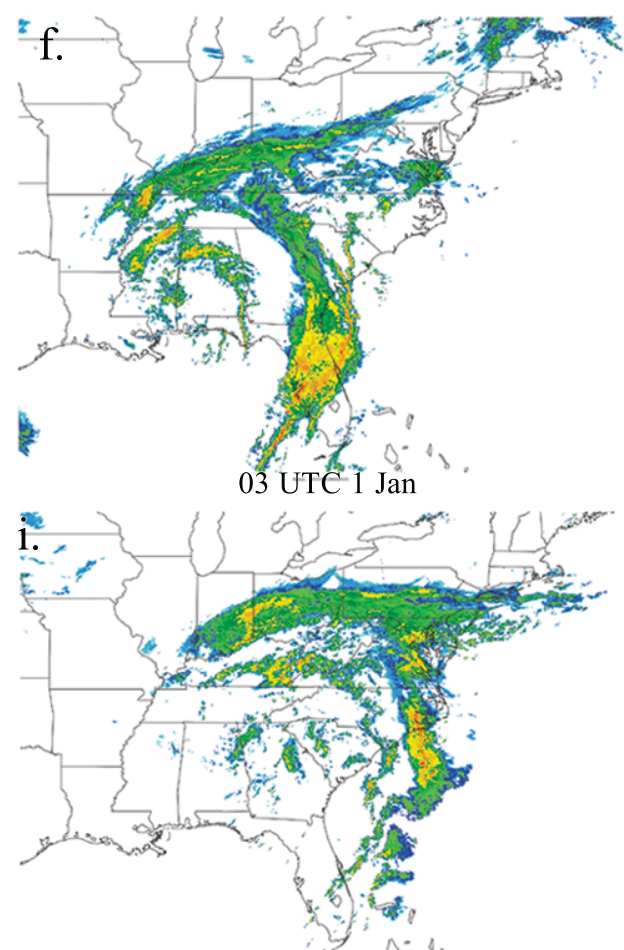

Figure 3.2. Radar mosaic imagery valid at: (a) 12 UTC 31 Dec; (b) 15 UTC 31 Dec (due to missing data, image obtained from NCDC online NEXRAD radar archive); (c) 18 UTC 31 Dec; (d) 21 UTC 31 Dec; (e) 00 UTC 1 Jan; (f) 03 UTC 1 Jan; (g) 06 UTC 1 Jan; (h) 09 UTC 1 Jan; (i) 12 UTC 1 Jan. 

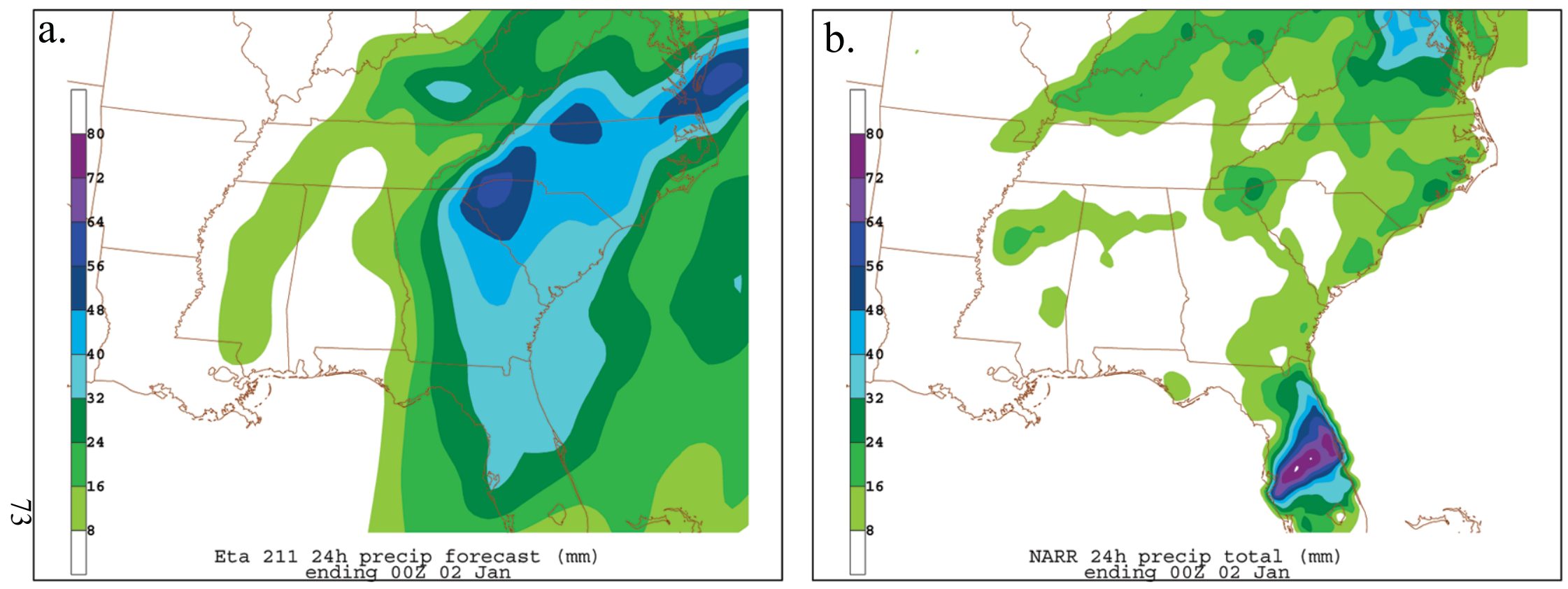

Figure 3.3 (a) 24-h total precipitation forecast ending 00 UTC 02 Jan by operational Eta model (mm, shaded as in colorbar in lower right corner; (b) 24-h NARR analysis precipitation totals ending 00 UTC 02 Jan (mm, shaded as in colorbar in lower right corner.) 

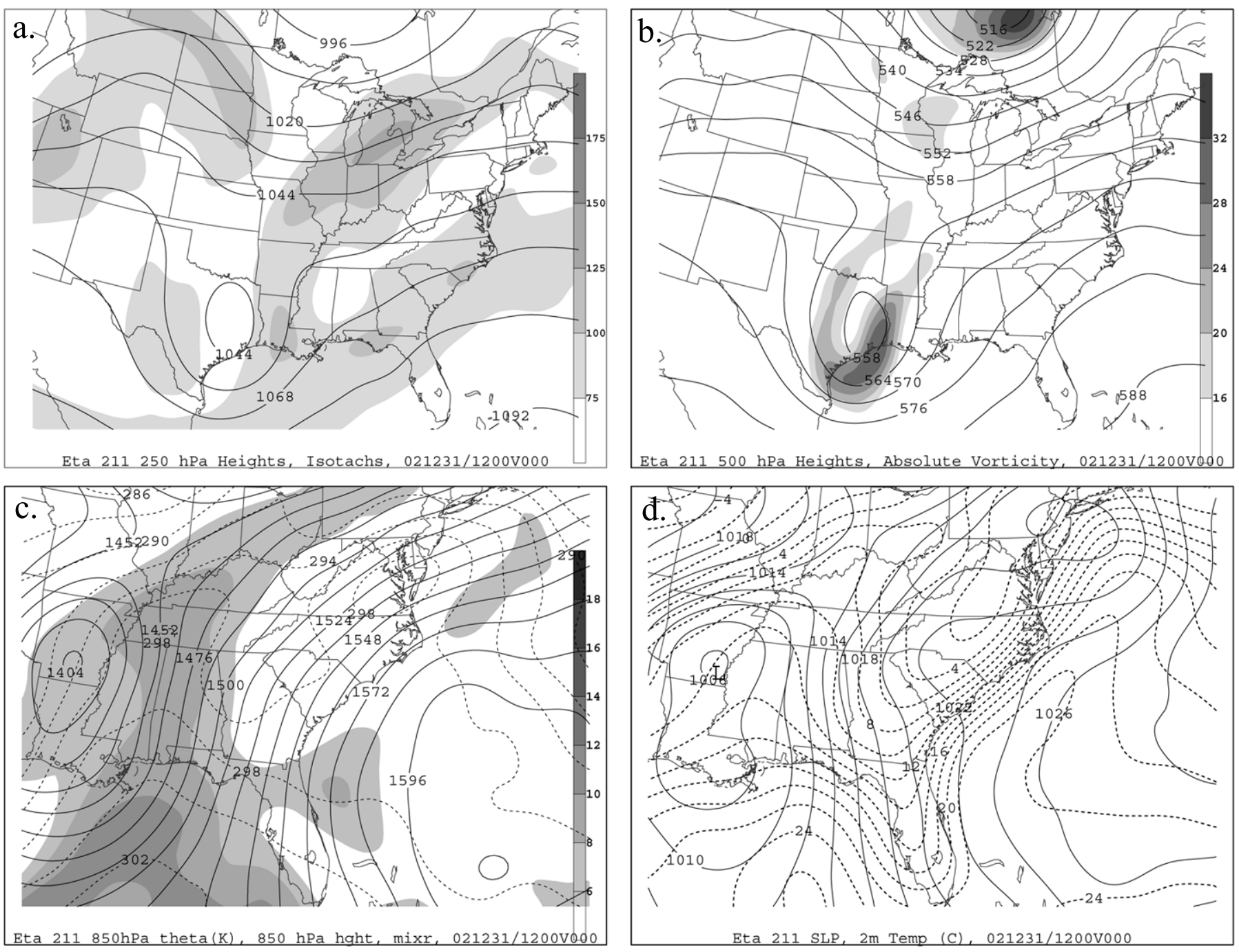

Figure 3.4. Eta Data Assimilation System (EDAS) analysis synoptic summary for 12 UTC 31 Dec 2002: (a) 250-hPa Geopotential height (solid contours, interval $12 \mathrm{dam}$ ) and isotachs (kt, shaded as in legend at right of panel); (b) $500-\mathrm{hPa}$ Geopotential height (solid, interval $6 \mathrm{dam}$ ) and vorticity ( $\mathrm{x} 10^{\wedge}-5 \mathrm{~s}^{\wedge}-1$, shaded as in legend at right of panel); (c) 850-hPa potential temperature (dashed contours, interval 2K ), 850-hPa Geopotential height (solid, interval $12 \mathrm{dam}$ ), and 850-hPa mixing ratio (shaded, interval $2 \mathrm{~g} / \mathrm{kg}$, values below $6 \mathrm{~g} / \mathrm{kg}$ omitted); (d) sea level pressure (solid contours, interval $2 \mathrm{hPa}$ ) and 2-m temperature (dashed contours, interval 2C). 

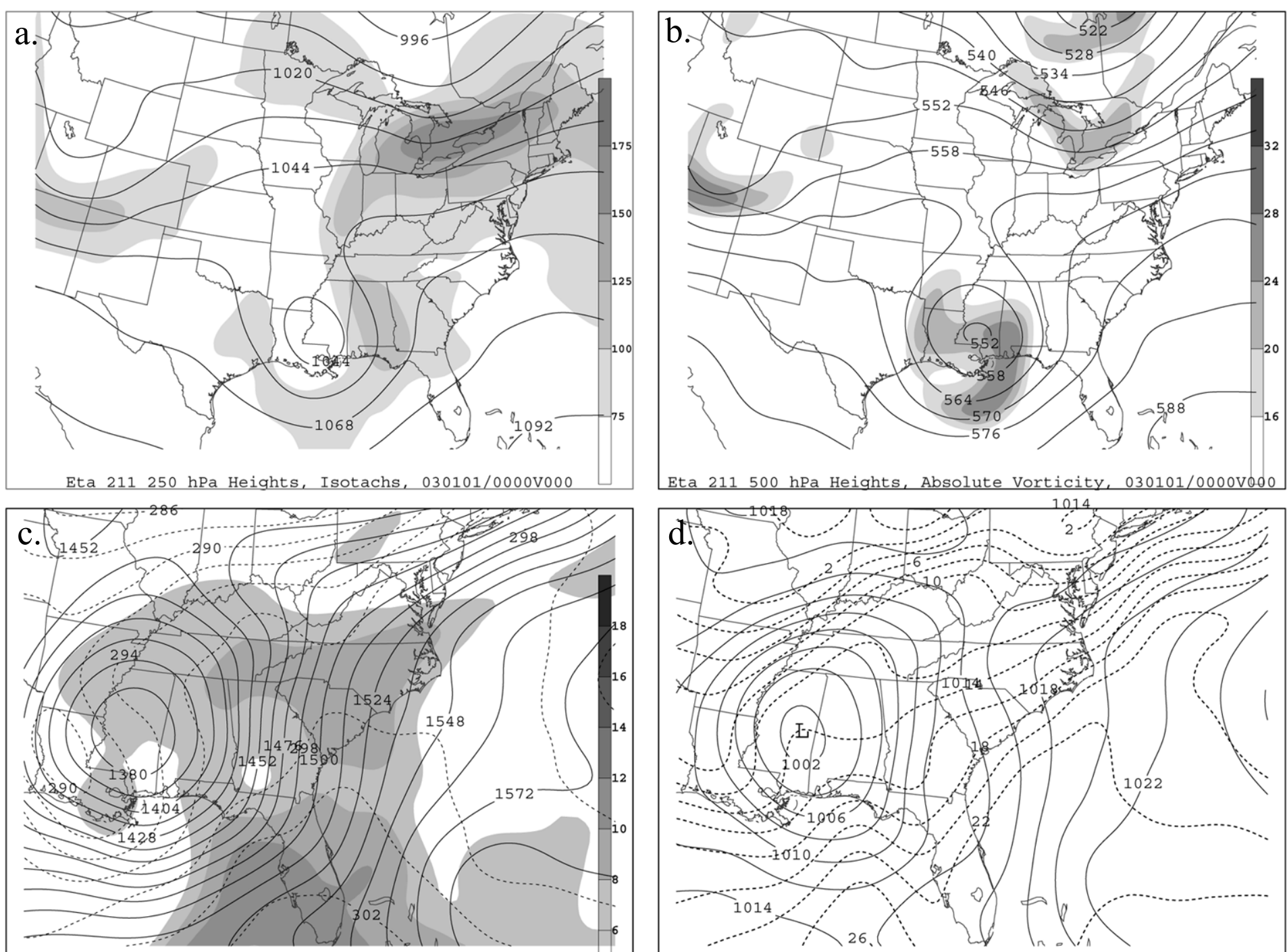

Eta $211850 \mathrm{hPa}$ theta $(K), 850 \mathrm{hPa}$ hght, mixr, $030101 / 0000 \mathrm{v} 000$ Figure 3.5. As in Fig. 3.4, except for 00 UTC 01 Jan 2003.

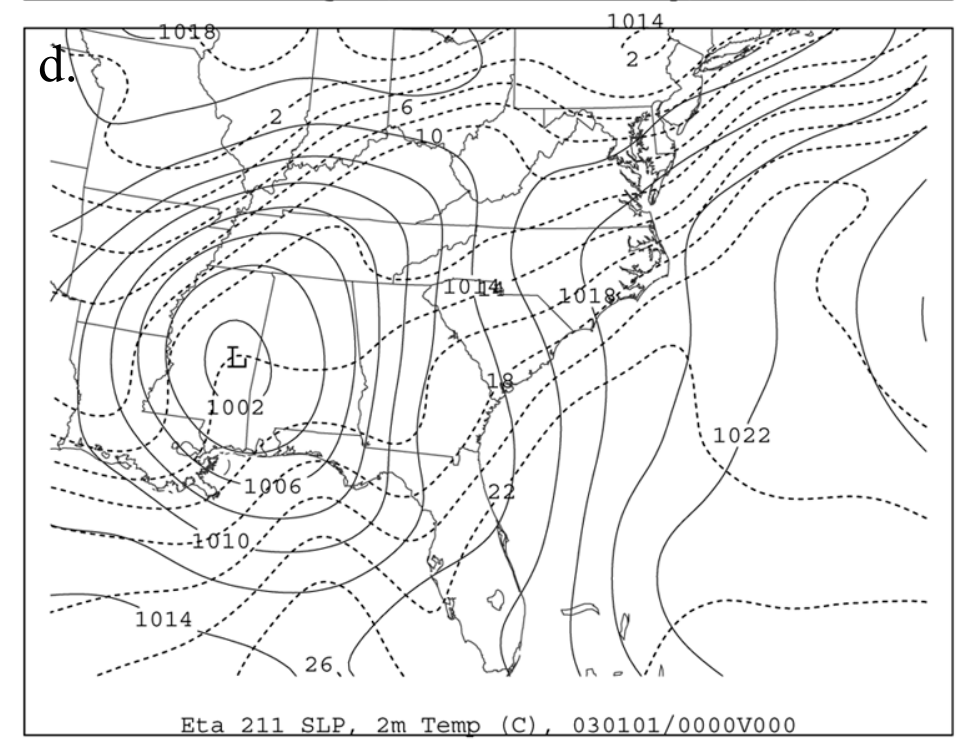



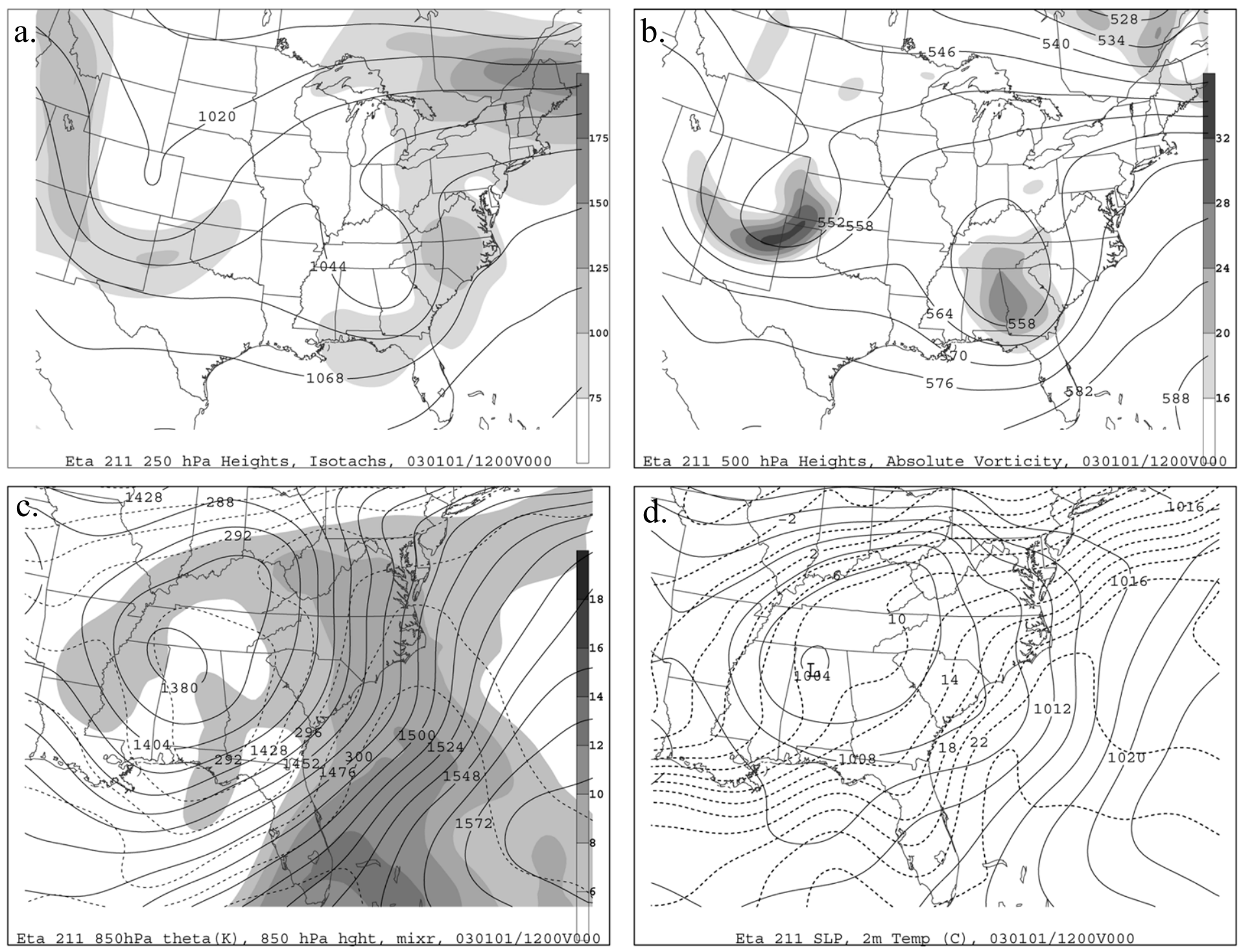

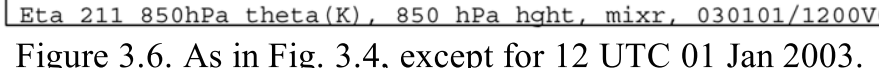



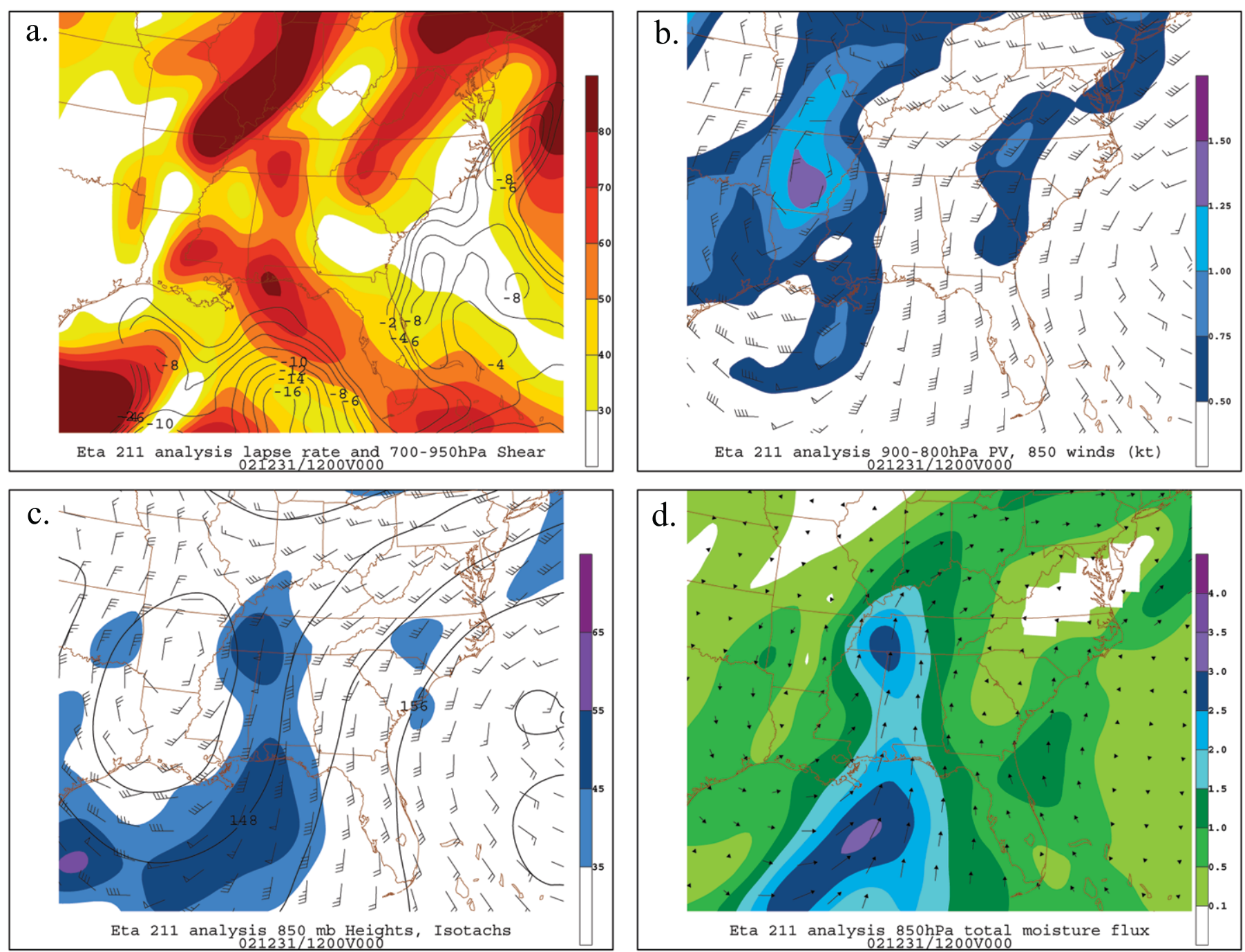

Figure 3.7. Eta Data Assimilation System (EDAS) analysis mesoscale summary for 12 UTC 31 Dec 2002: (a) 500-950-hPa equivalent potential temperature difference (solid contours, interval $2 \mathrm{~K}$, positive values omitted) and 700-950-hPa wind shear (10 kt/m, shaded as in legend at right of panel); (b) 900-800 layer-averaged PV (PVU, shaded as in legend at right of panel) and $850-\mathrm{hPa}$ winds (kt, barbs); (c) $850-\mathrm{hPa}$ Geopotential height (solid, interval 4 dam), isotachs (kt, shaded as in legend at right of panel), and 850-hPa winds (kt, barbs); (d) 850-hPa moisture flux (g kg-1 m s-1, shaded as in legend at right of panel) and moisture flux vectors (arrows). 


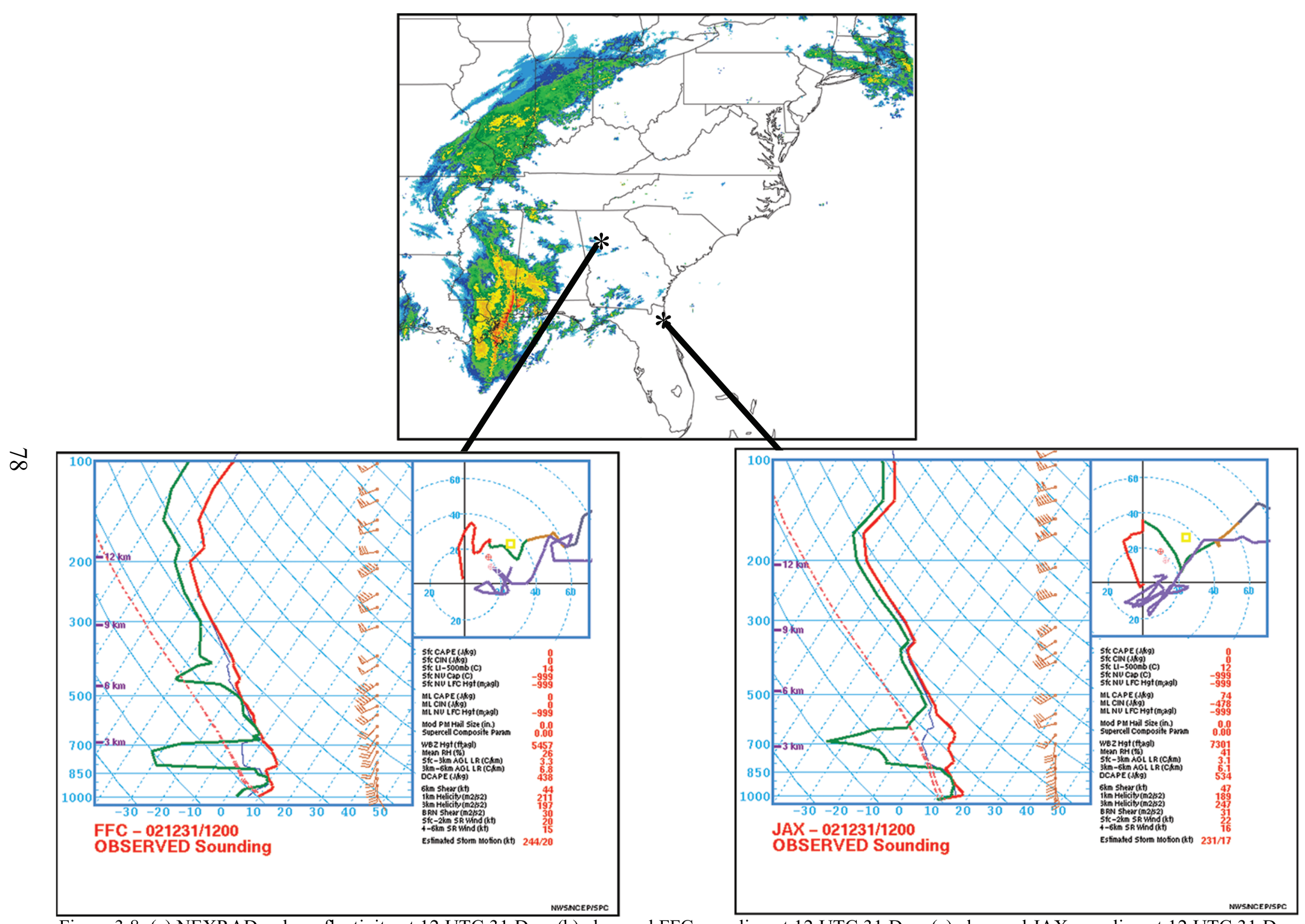

Figure 3.8. (a) NEXRAD radar reflectivity at 12 UTC 31 Dec; (b) observed FFC sounding at 12 UTC 31 Dec; (c) observed JAX sounding at 12 UTC 31 Dec. 

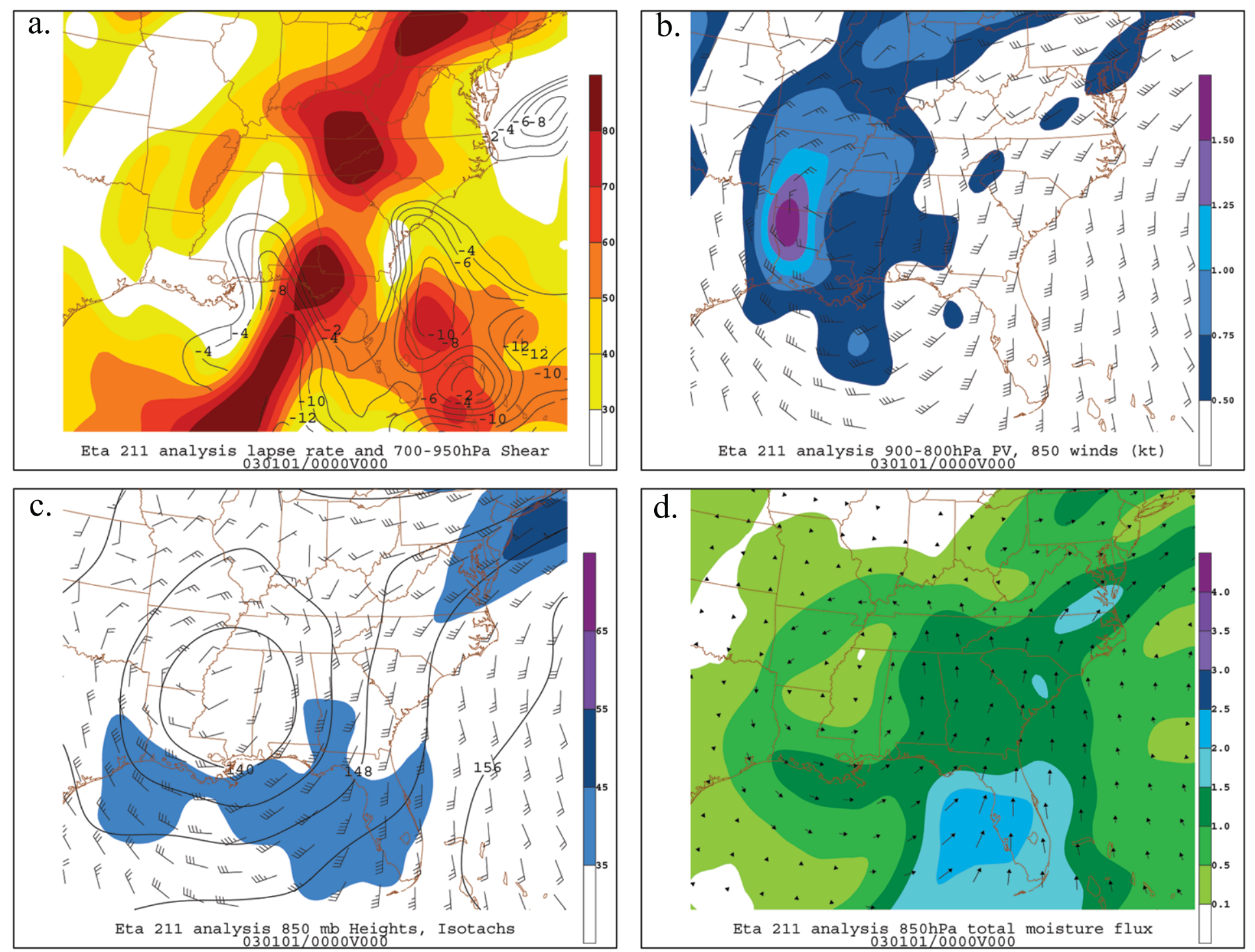

Figure 3.9. As in Fig. 3.7, except for 00 UTC 01 Jan 2003. 

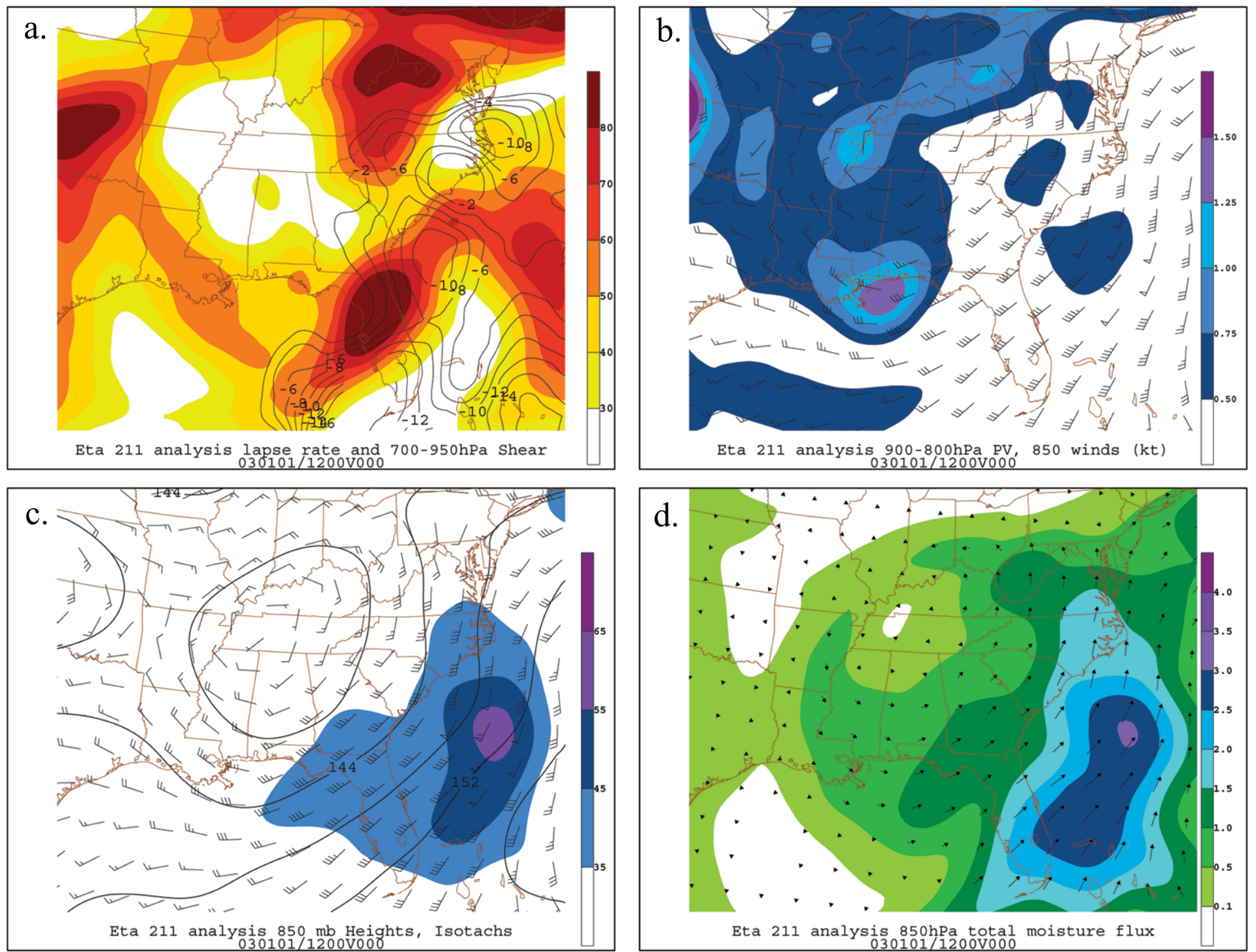

Figure 3.10. As in Fig. 3.8, except for 12 UTC 01 Jan 2003. 

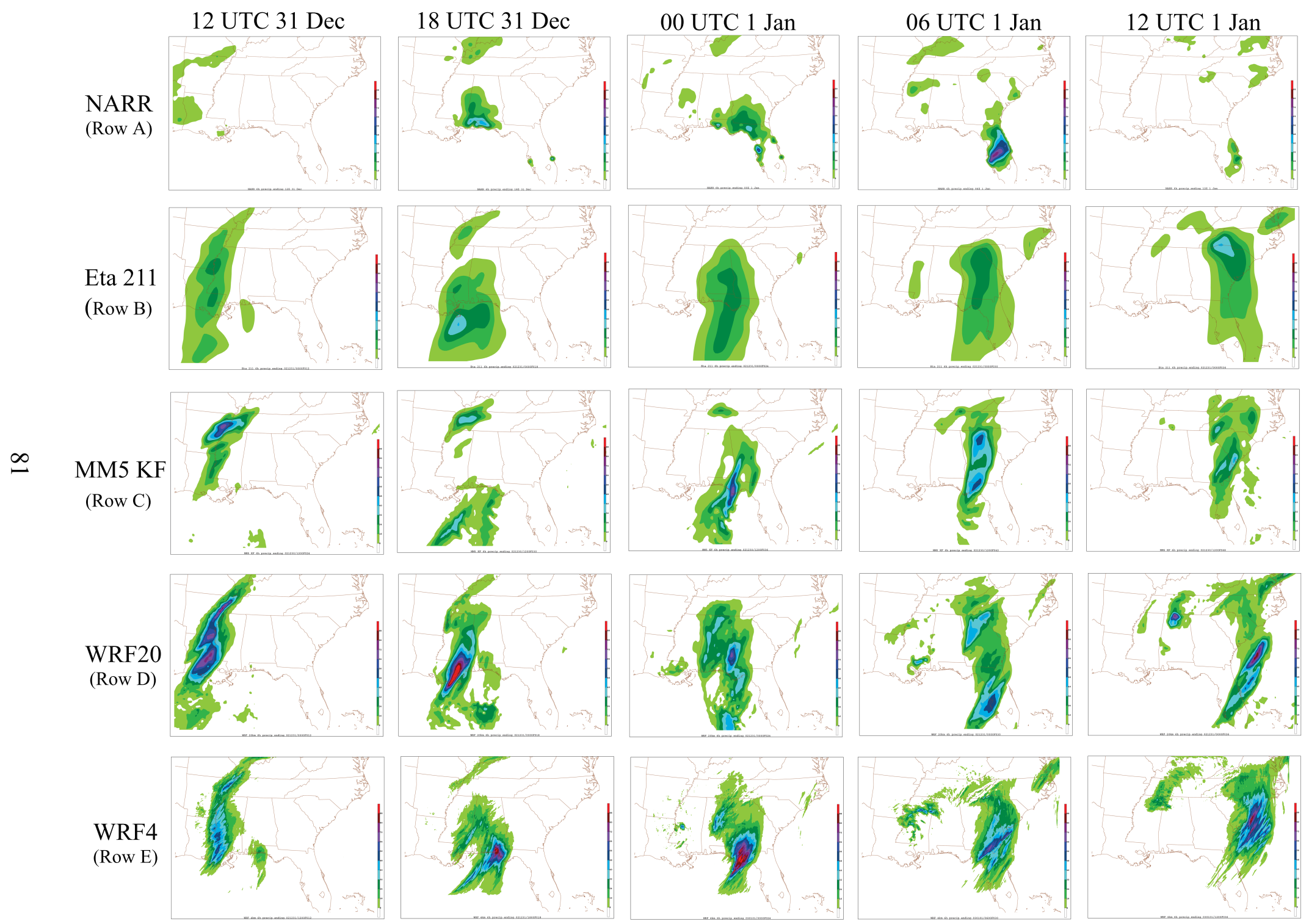

Figure 3.11. (Row A) (from left to right): NARR 6-h precipitation analyses ending at 12 UTC 31 Dec, 18 UTC 31 Dec, 00 UTC 01 Jan, 06 UTC 01 Jan, 12 UTC 01 Jan, respectively; (Row B) as in (Row A) except for operational Eta forecast; (Row C) as in (Row A) except for MM5 KF model simulation; (Row D) as in (Row A) except for WRF20 forecast; (Row E) as in (Row A) except for WRF4 forecast. 


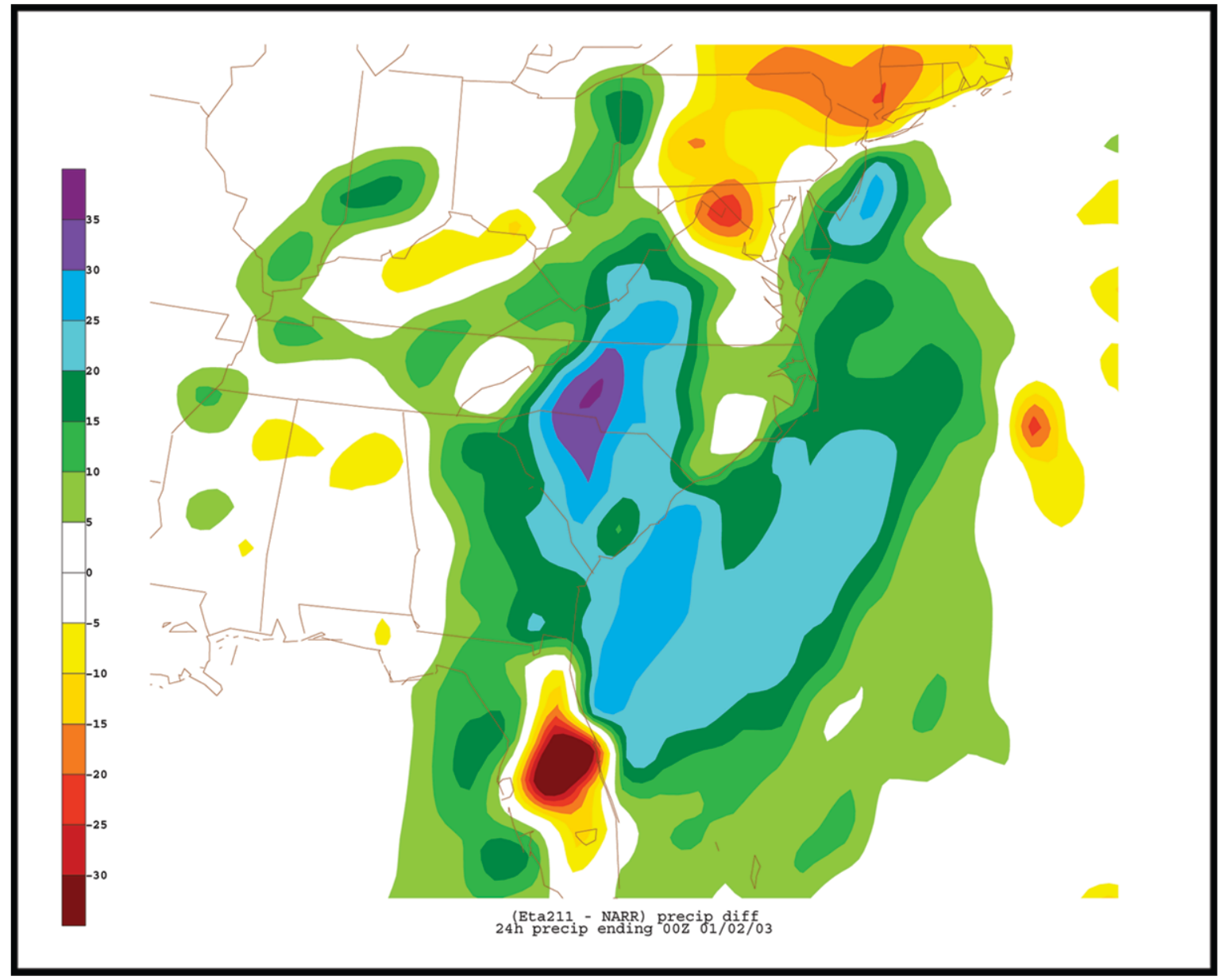

Figure 3.12. Difference field between Eta 211 24-h QPF and NARR analysis 24-h total from 24-h period ending 00 UTC $02 \mathrm{Jan}$ (mm, shaded as in legend at left of panel). 

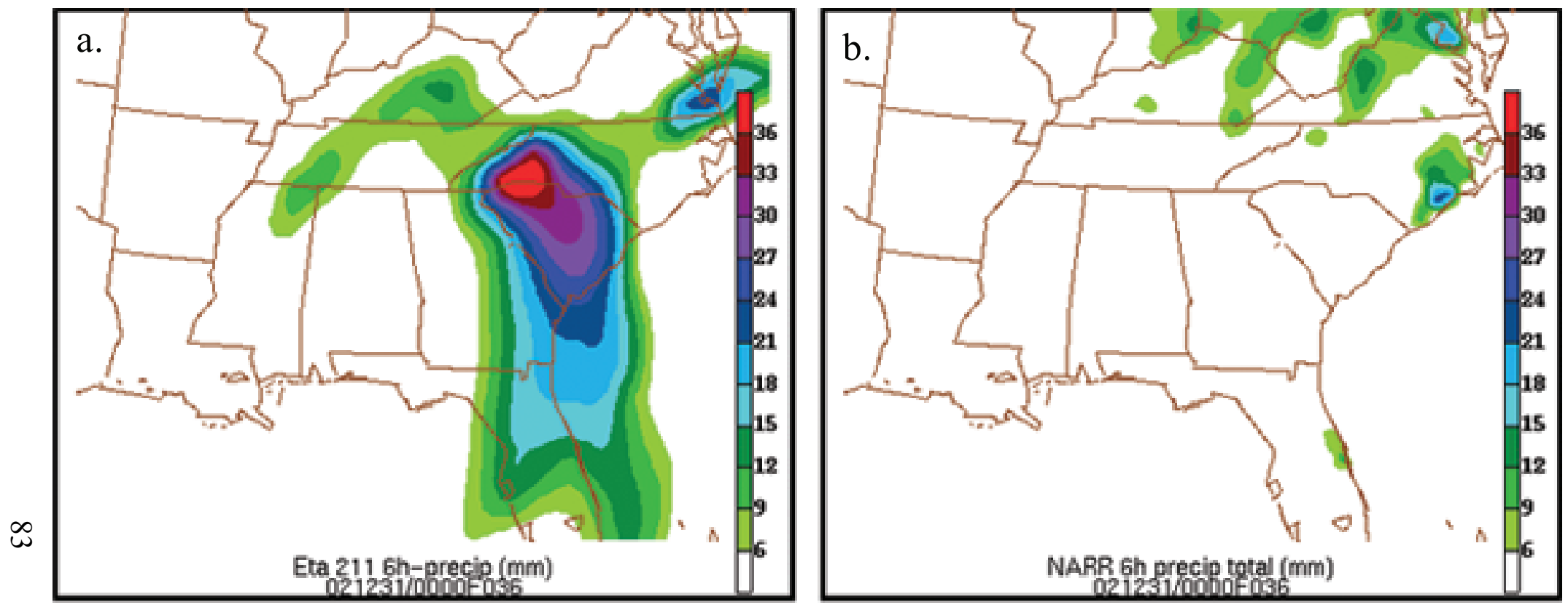

Figure 3.13. (a) 6-h total precipitation forecast by operational Eta model ending 12 UTC 1 Jan (mm, shaded as in legend at right of panel; (b) 6-h NARR analy sis precipitation totals ending 12 UTC 1 Jan (mm, shaded as in legend at right of panel). 

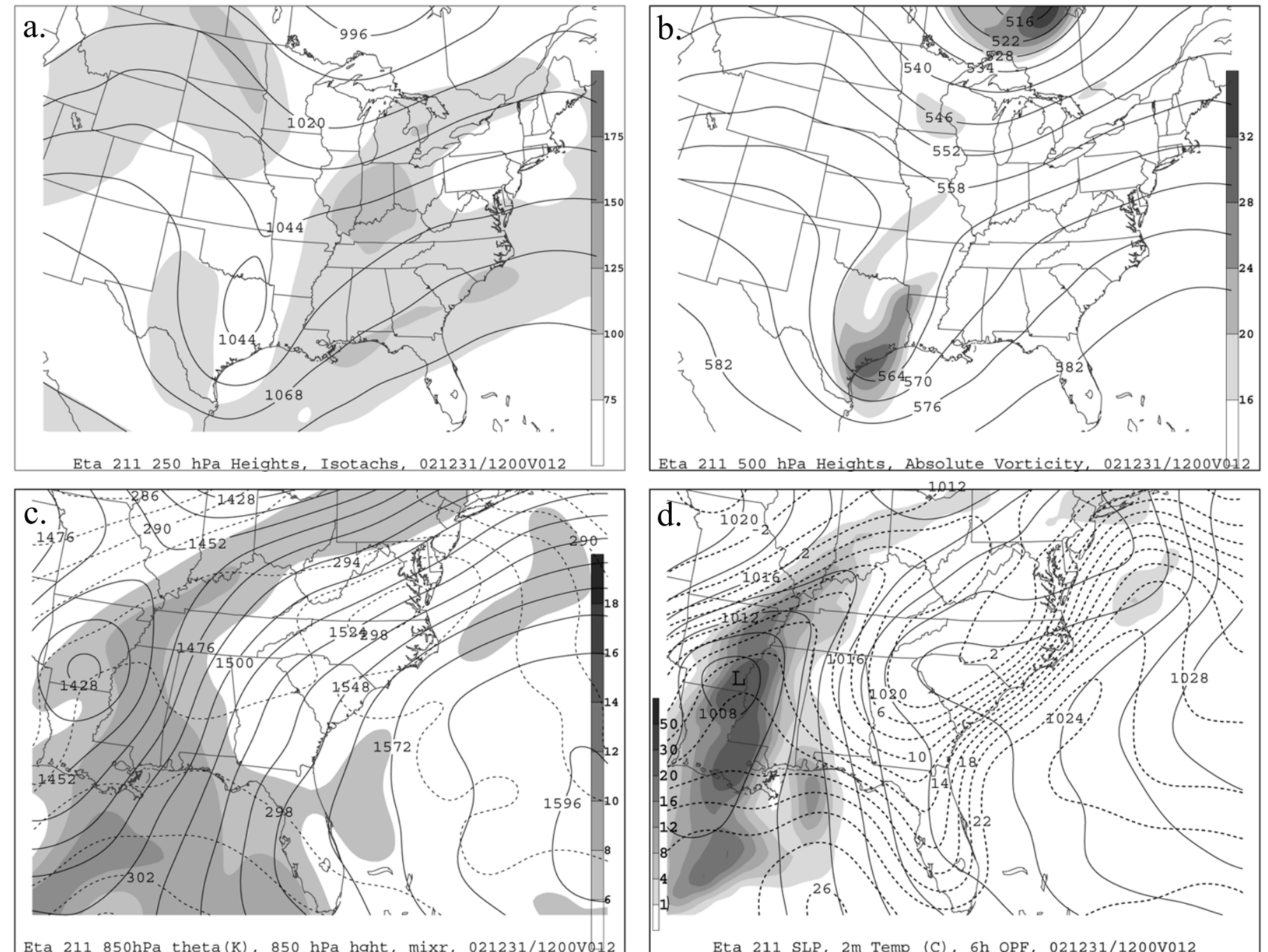

Figure 3.14. Operational Eta 12-h forecast valid 12 UTC 31 Dec 2002: (a) 250-hPa Geopotential height (solid contours, interval 12 dam) and isotachs (kt, shaded as in legend at right of panel); (b) 500-hPa Geopotential height (solid, interval $6 \mathrm{dam}$ ) and vorticity (, shaded as in legend at right of panel); (c) 850-hPa potential temperature (dashed contours, interval $2 \mathrm{~K}$ ), $850-\mathrm{hPa}$ Geopotential height (solid, interval $12 \mathrm{dam}$ ), and 850-hPa mixing ratio (shaded, interval $2 \mathrm{~g} / \mathrm{kg}$, values below $6 \mathrm{~g} / \mathrm{kg}$ omitted); (d) sea level pressure (solid contours, interval $2 \mathrm{hPa}$ ), 2-m temperature (dashed contours, interval 2C), and 6-h accumulated precipitation (mm, shaded as in legend at left of panel) ending 12 UTC 31 Dec. 

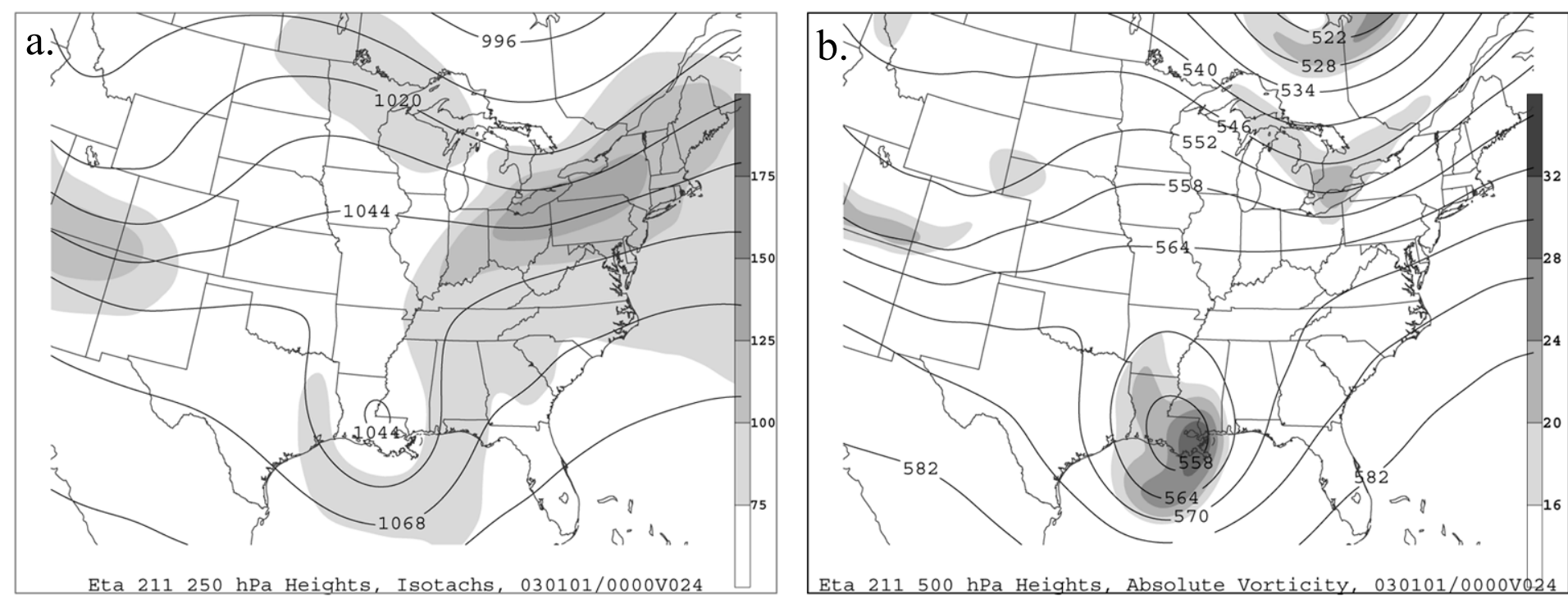

$\stackrel{\infty}{\varphi^{\prime}}$
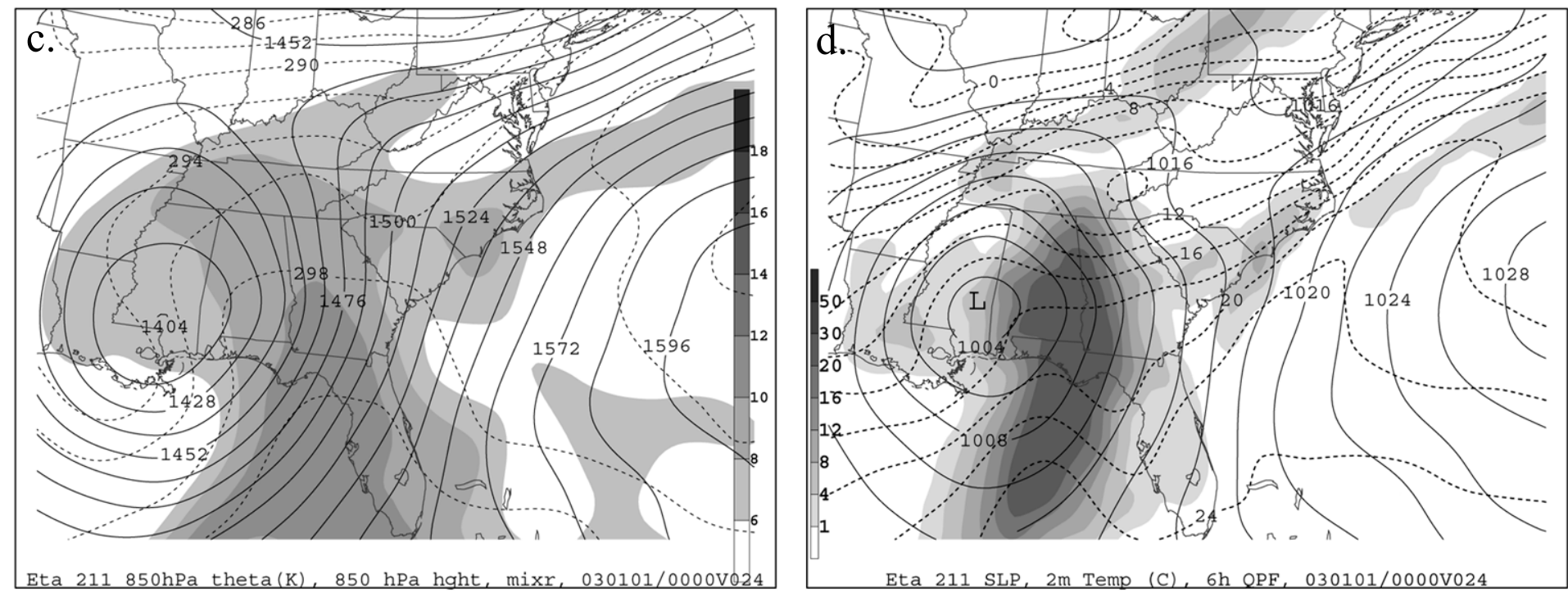

Figure 3.15. As in Fig. 3.14 except for 24-h forecast valid 00 UTC 1 Jan. 

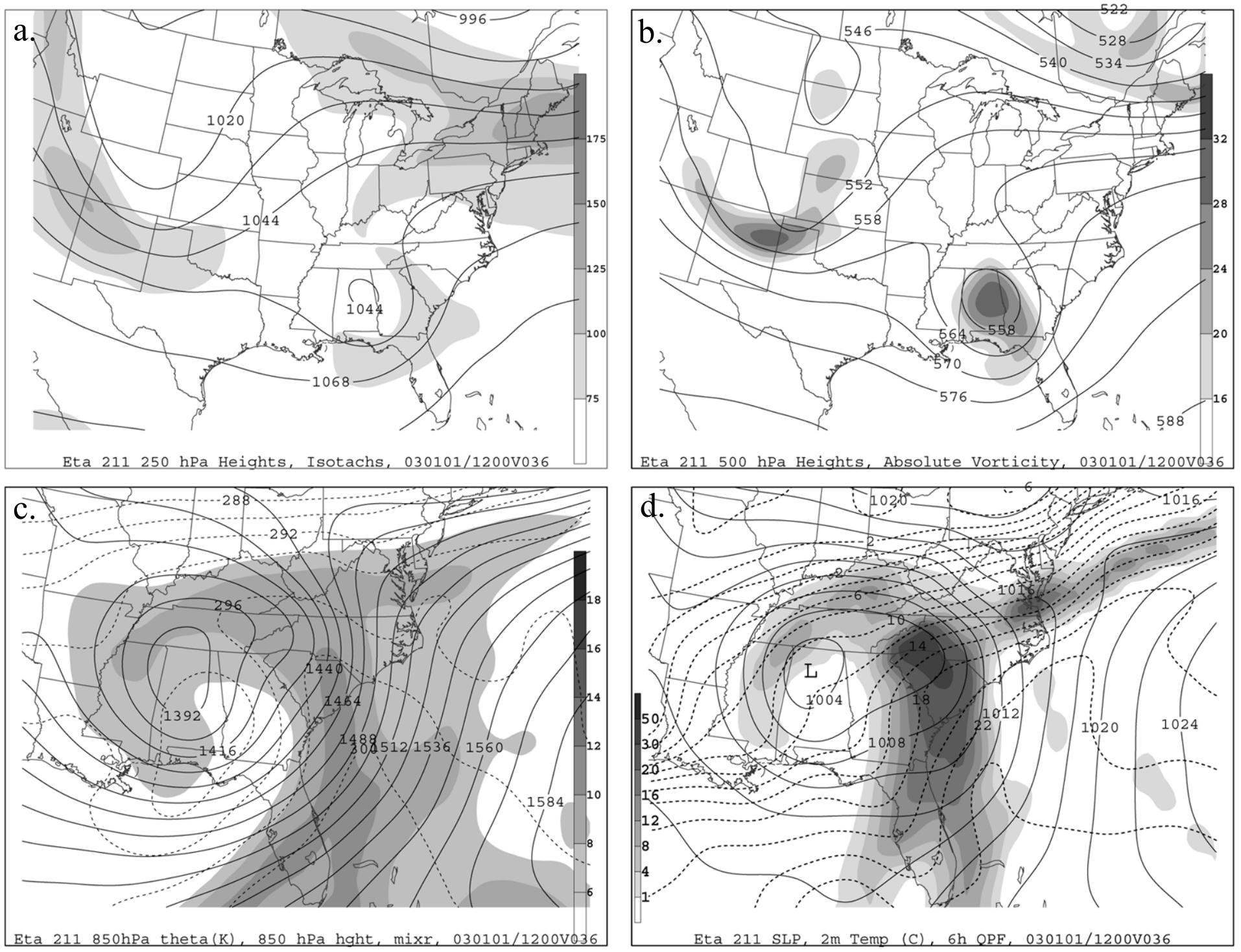

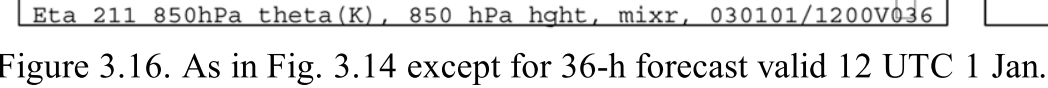



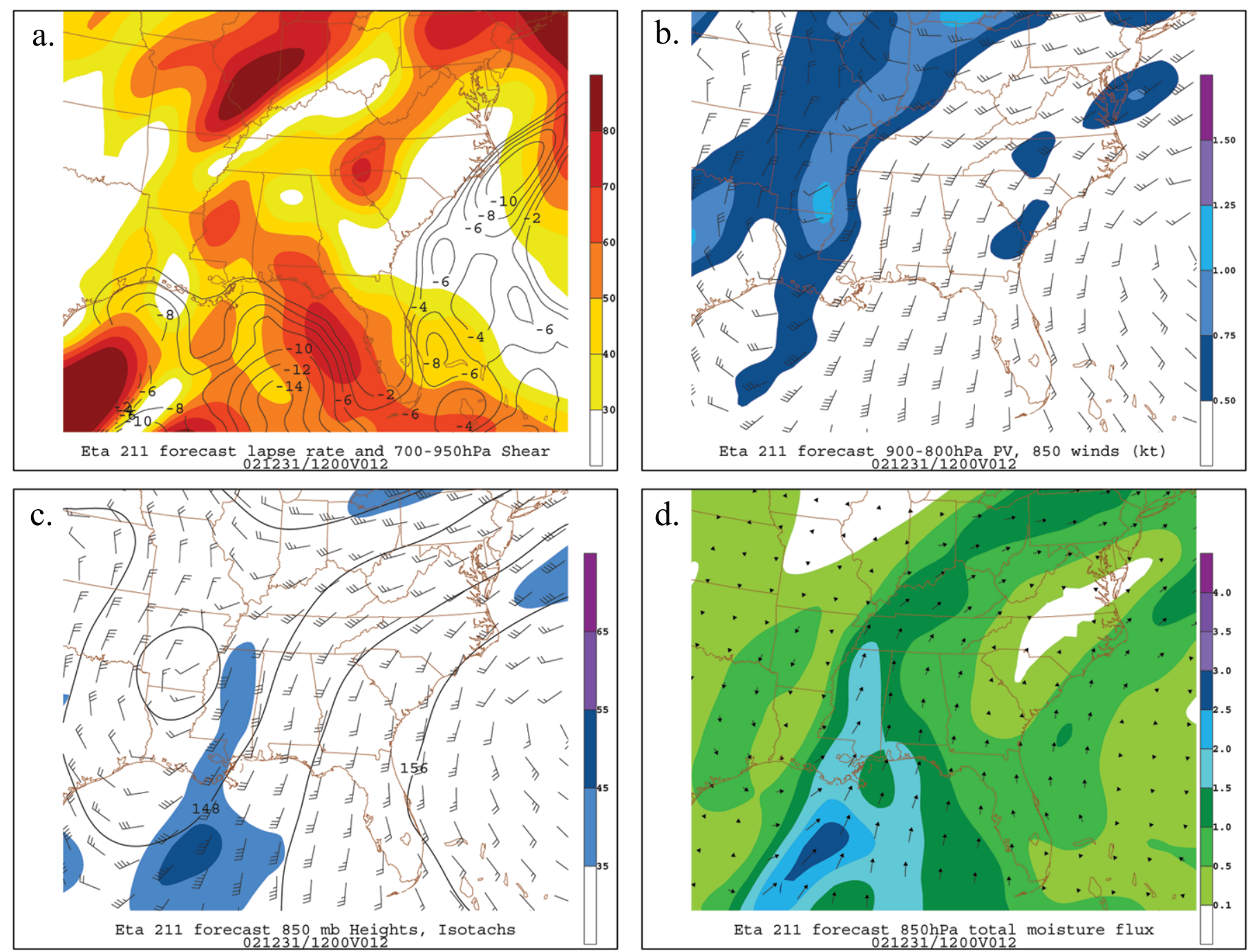

Figure 3.17. Operational Eta 12-h forecast valid 12 UTC 31 Dec 2002: (a) 500-950-hPa equivalent potential temperature difference (solid contours, interval $2 \mathrm{~K}$, positive values omitted) and 700-950-hPa wind shear (10 kt/m, shaded as in legend at right of panel); (b) 900-800 layer-averaged PV (PVU, shaded as in legend at right of panel) and 850-hPa winds (kt, barbs); (c) 850-hPa Geopotential height (solid contours, interval 4 dam), isotachs (kt, shaded as in legend at right of panel), and 850-hPa winds (kt, barbs); (d) 850-hPa moisture flux ( $\mathrm{g} \mathrm{kg}-1 \mathrm{~m} \mathrm{~s}-1$, shaded as in legend at right of panel) and moisture flux vectors (arrows). 

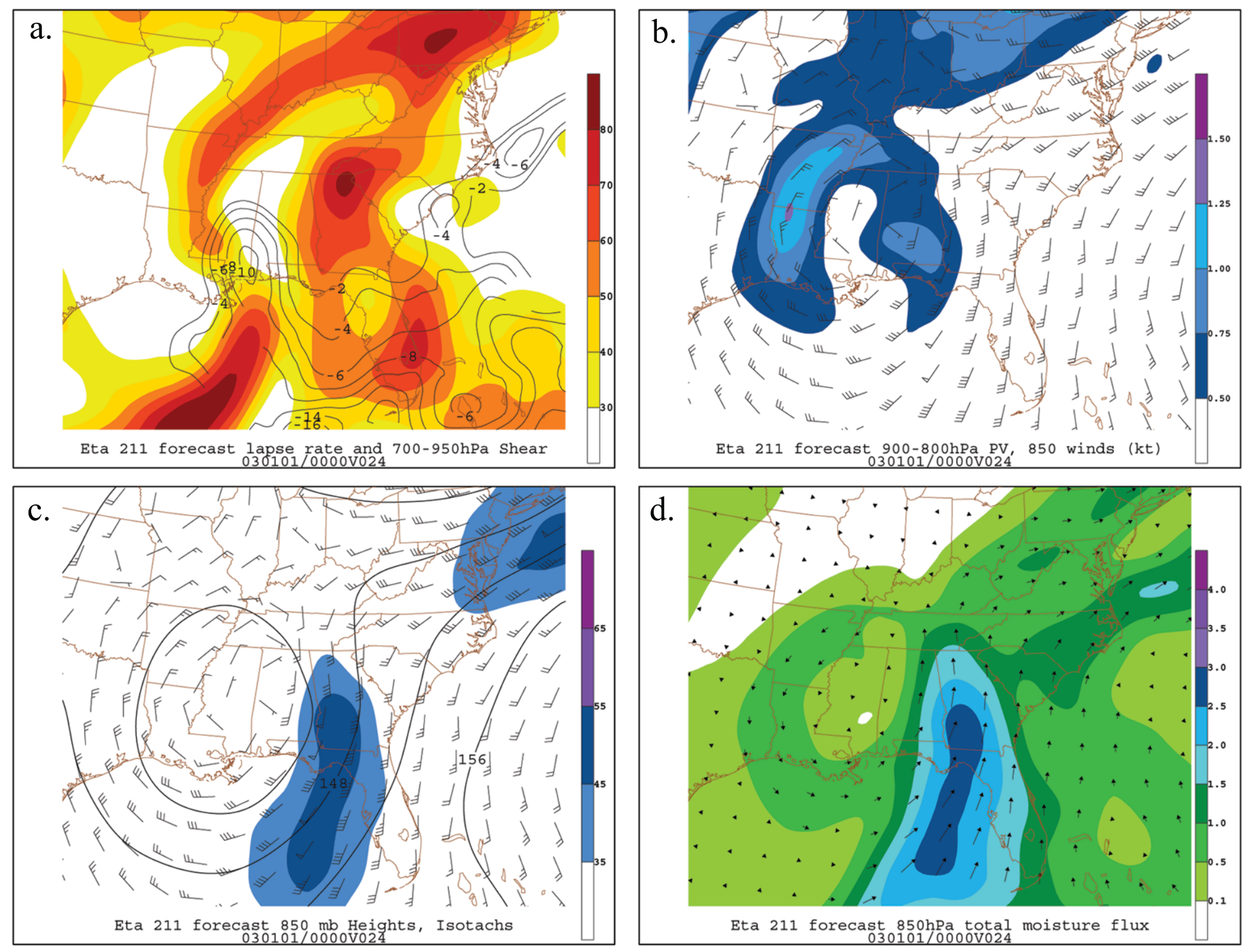

Figure 3.18. As in Fig. 3.17 except for 24-h forecast valid 00 UTC 1 Jan. 

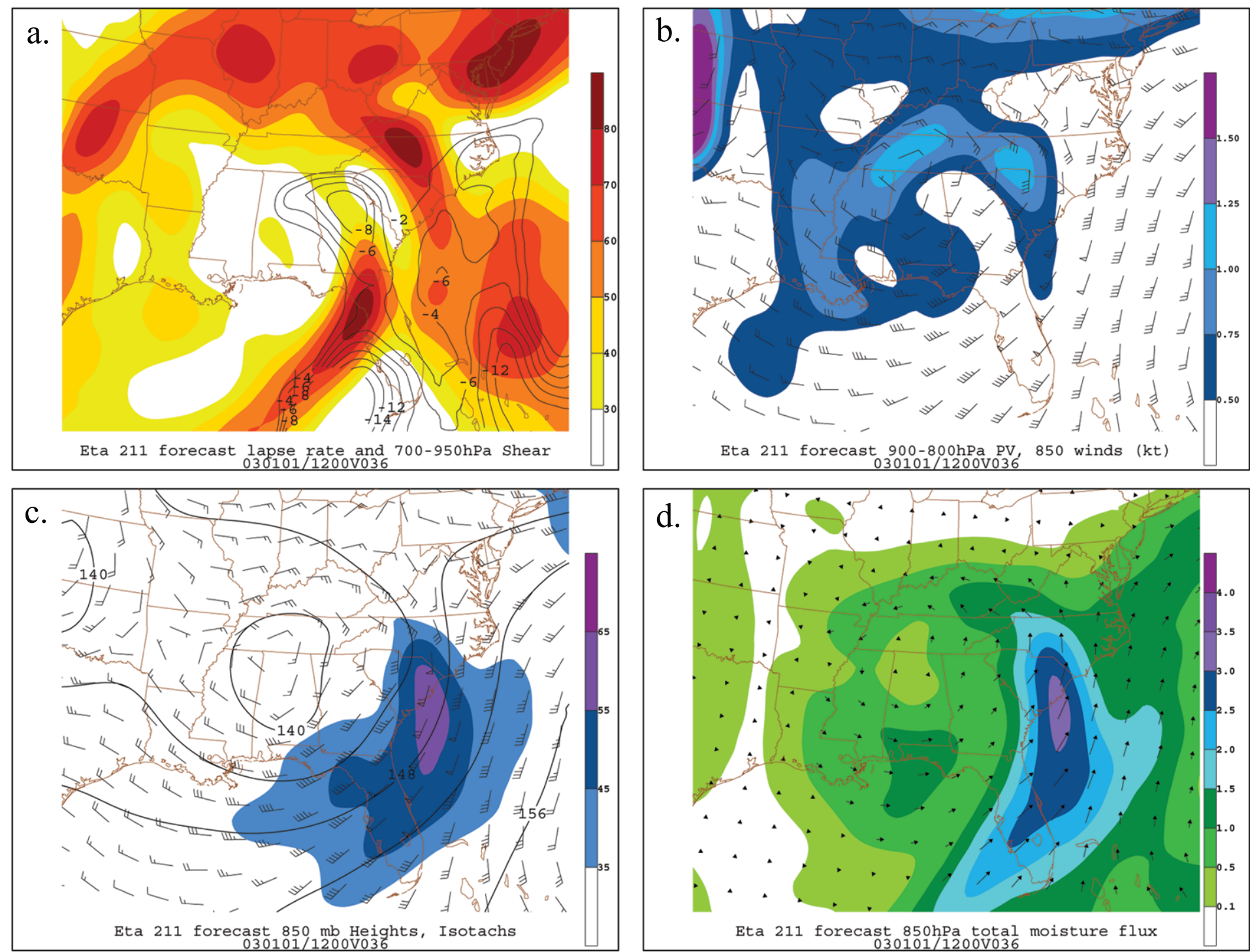

Figure 3.19. As in Fig. 3.17 except for 36-h forecast valid 12 UTC 1 Jan. 

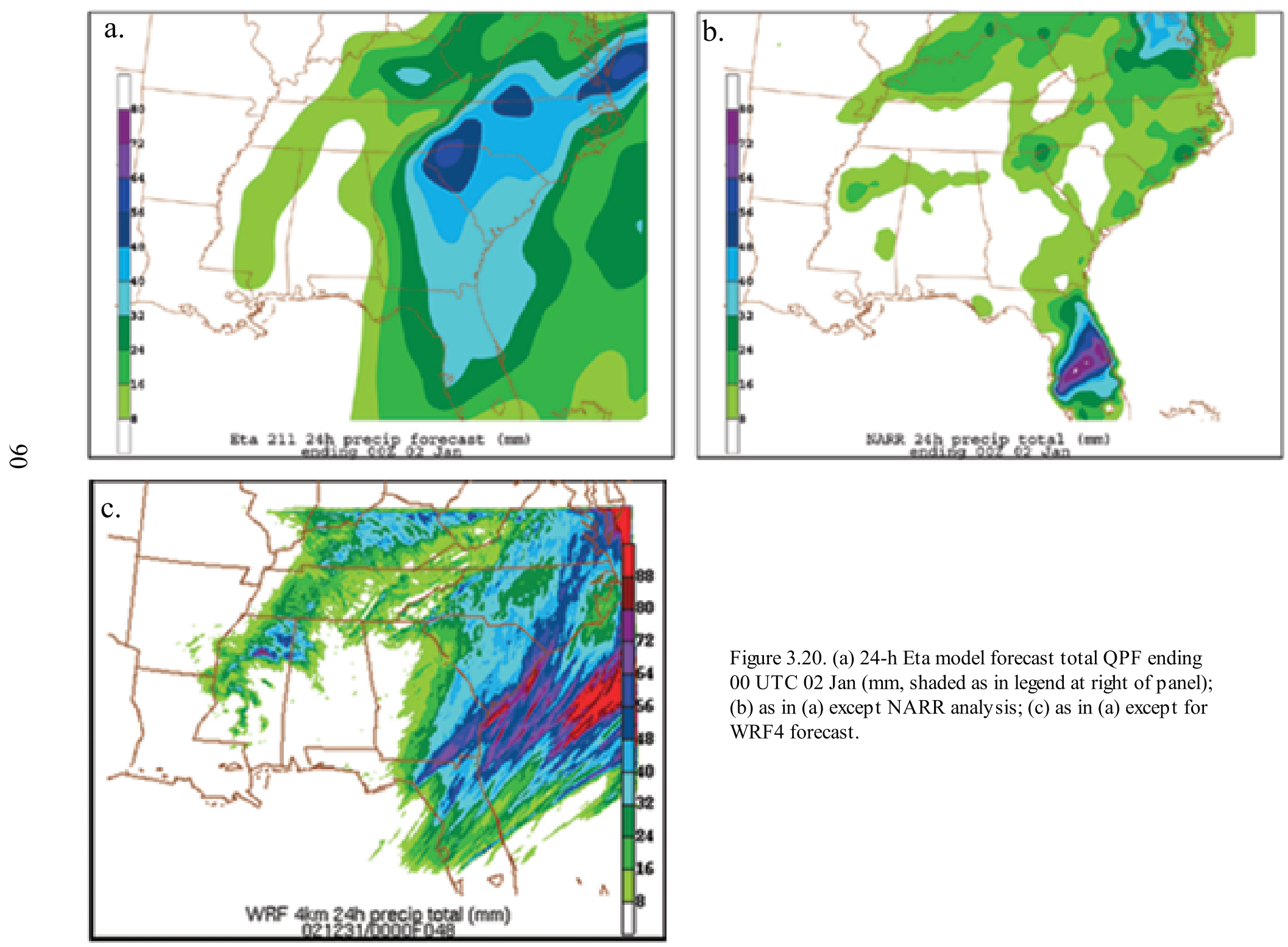

Figure 3.20. (a) 24-h Eta model forecast total QPF ending 00 UTC $02 \mathrm{Jan}$ (mm, shaded as in legend at right of panel); (b) as in (a) except NARR analy sis; (c) as in (a) except for WRF4 forecast. 

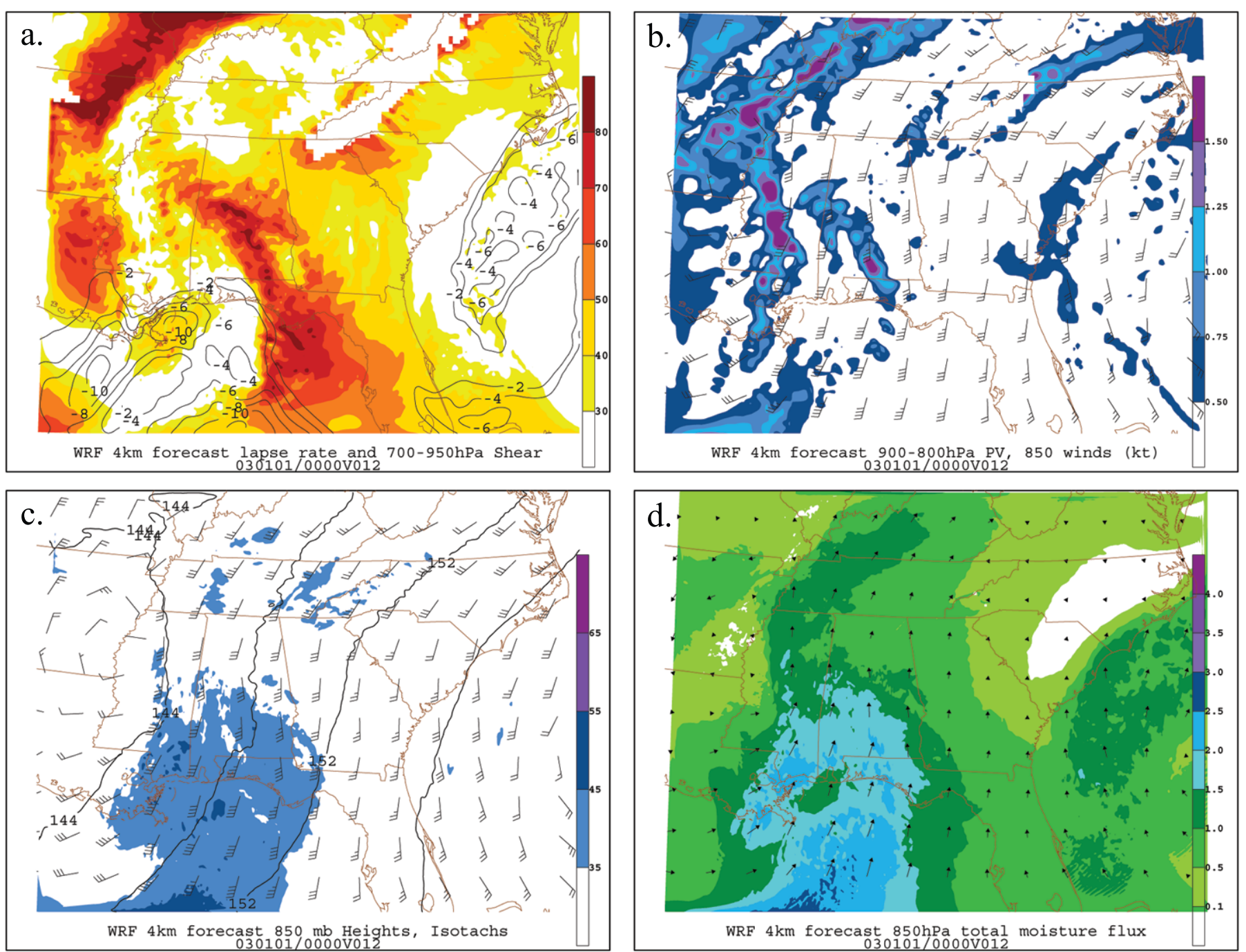

Figure 3.21. WRF4 12-h forecast valid 12 UTC 31 Dec 2002: (a) 500-950-hPa equivalent potential temperature difference (solid contours, interval 2 K, positive values omitted) and 700-950-hPa wind shear (10 kt/m, shaded as in legend at right of panel); (b) 900-800 layer-averaged PV (PVU, shaded as in legend at right of panel) and 850-hPa winds (kt, barbs); (c) 850-hPa Geopotential height (solid, interval 4 dam), isotachs (kt, shaded as in legend at right of panel), and 850-hPa winds (kt, barbs); (d) 850 -hPa moisture flux ( $\mathrm{gg}-1 \mathrm{~m} \mathrm{~s}-1$, shaded as in legend at right of panel) and moisture flux vectors (arrows). 

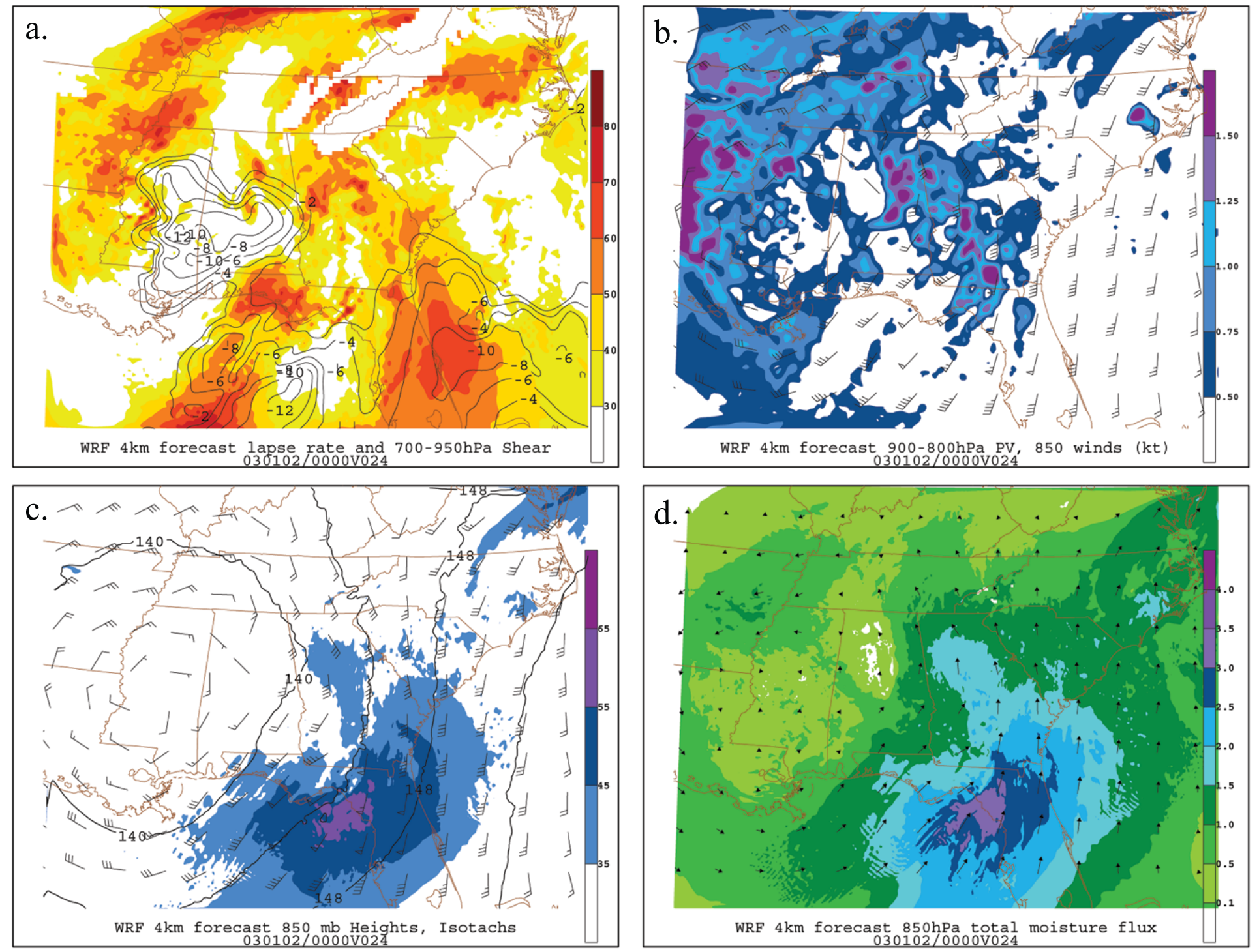

Figure 3.22. As in Fig. 3.21 except for 24-h forecast valid 00 UTC 1 Jan. 

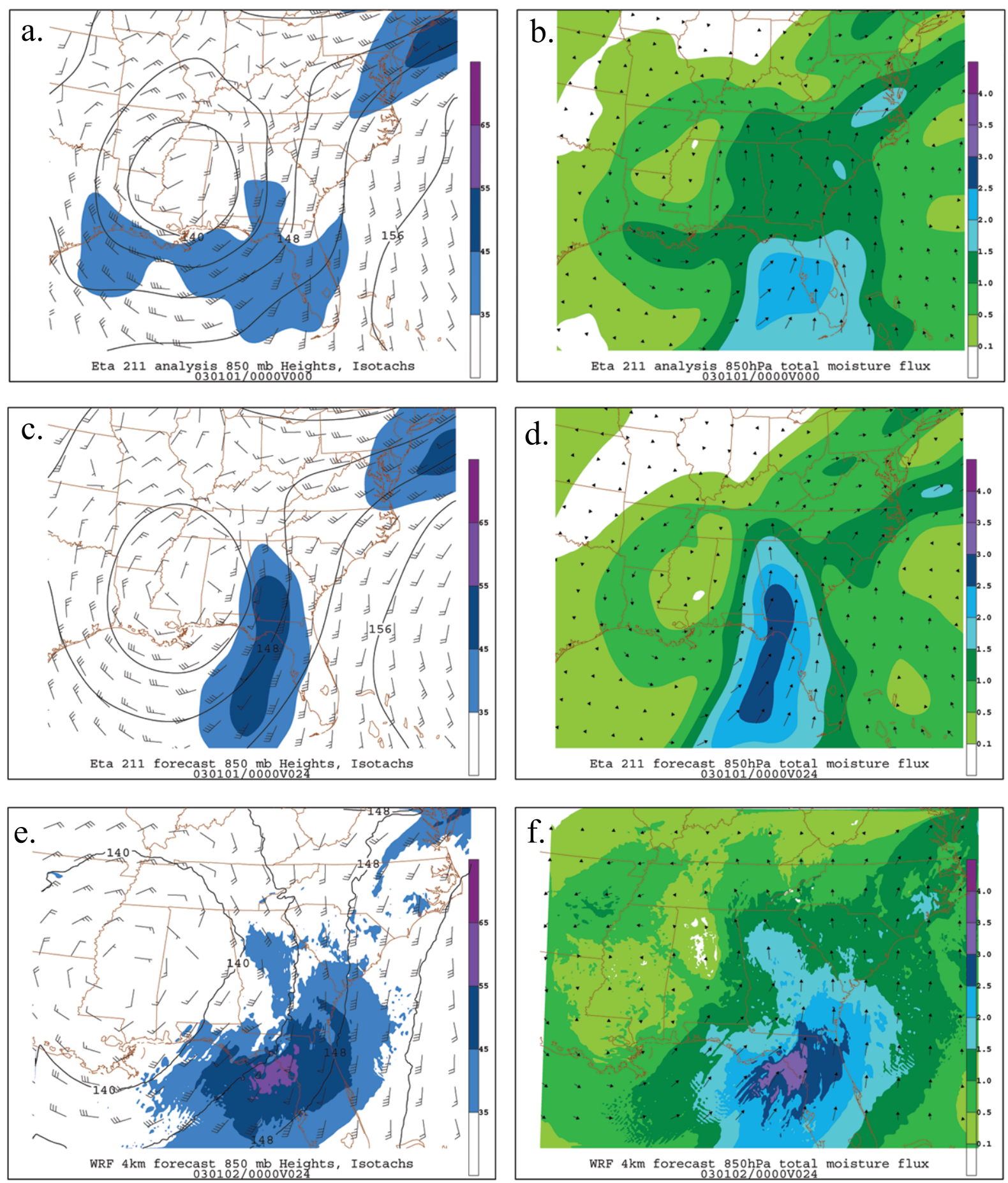

Figure 3.23. (a) 850-hPa Geopotential height (solid contours, interval 4 dam), 850-hPa winds (kt, barbs), and 850-hPa isotachs (kt, shaded as in legend at right of panel) for Eta analysis valid 00 UTC 1 Jan; (b) 850-hPa moisture flux magnitude (g kg-1 m s-1, shaded as in legend at right of panel) and moisture flux vectors (arrows) for Eta analysis valid 00 UTC 1 Jan; (c) as in (a), except for 12-h Eta forecast; (d) as in (b), except for 12-h Eta forecast, (e) as in (a), except for 12-h WRF4 forecast, (f) as in (b), except for 12-h WRF4 forecast. 

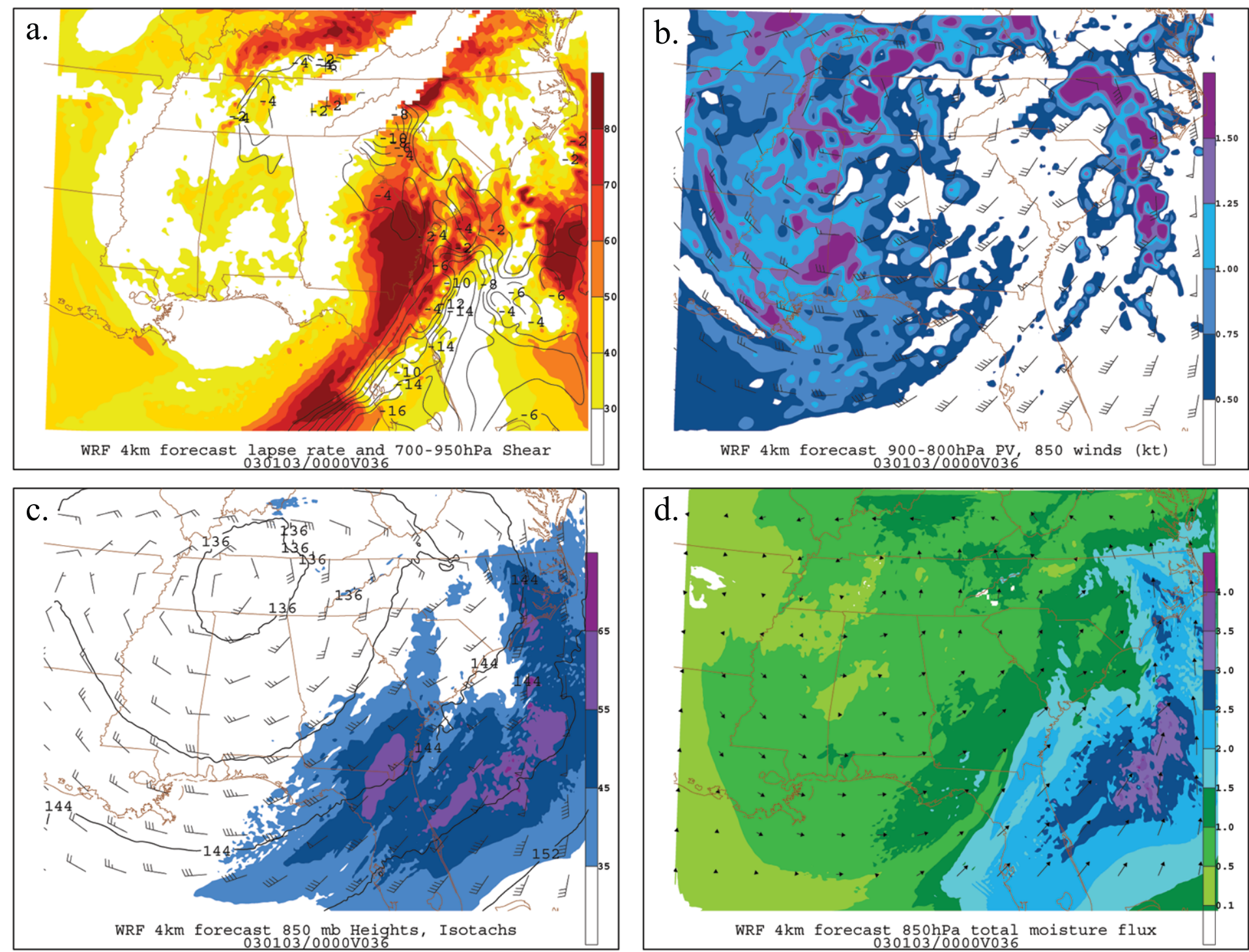

Figure 3.24. As in Fig. 3.21 except for 36-h forecast valid 12 UTC 1 Jan. 

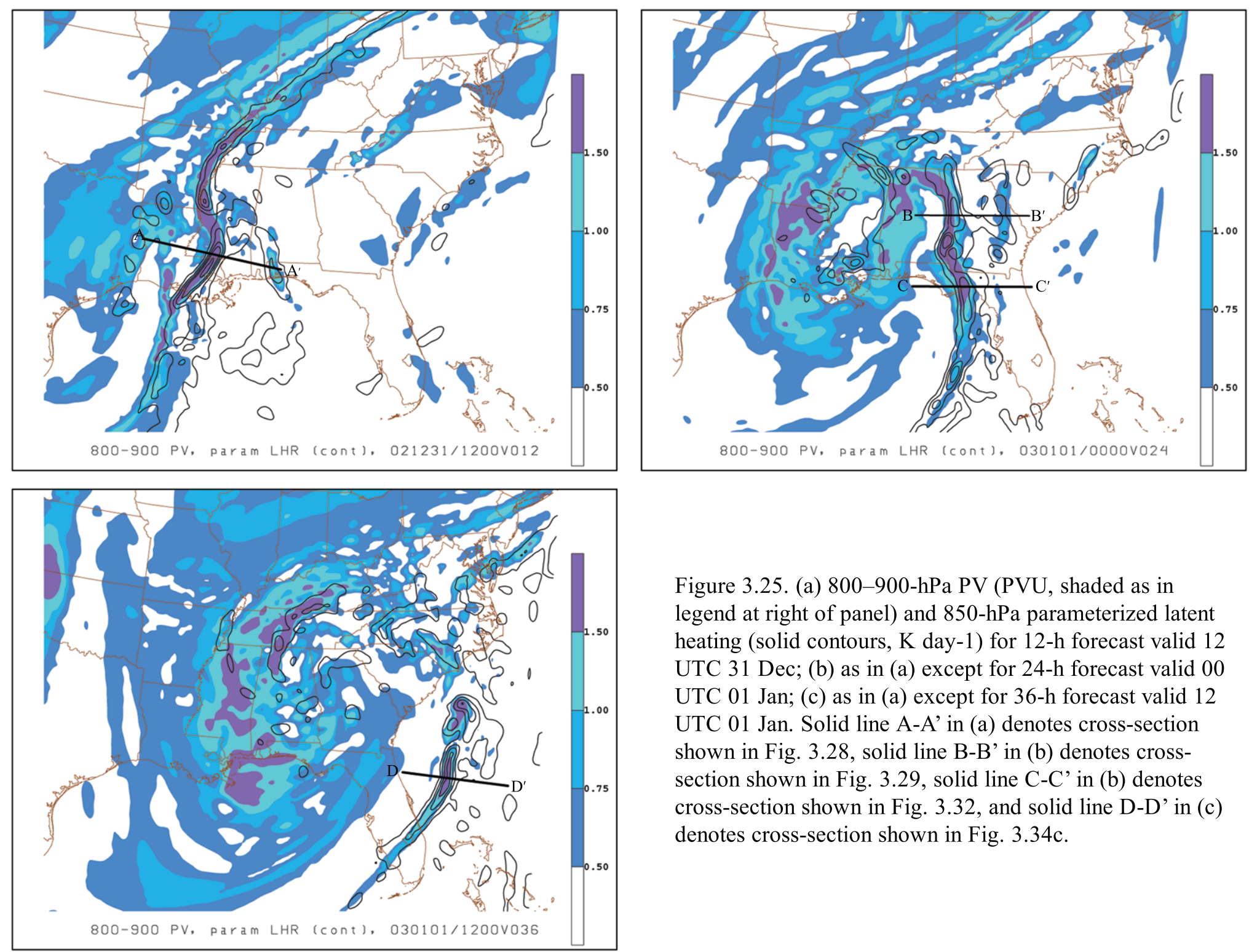

Figure 3.25. (a) 800-900-hPa PV (PVU, shaded as in legend at right of panel) and $850-\mathrm{hPa}$ parameterized latent heating (solid contours, $\mathrm{K}$ day-1) for 12-h forecast valid 12 UTC 31 Dec; (b) as in (a) except for 24-h forecast valid 00 UTC 01 Jan; (c) as in (a) except for 36-h forecast valid 12 UTC 01 Jan. Solid line A-A' in (a) denotes cross-section shown in Fig. 3.28, solid line B-B' in (b) denotes crosssection shown in Fig. 3.29, solid line C-C' in (b) denotes cross-section shown in Fig. 3.32, and solid line D-D' in (c) denotes cross-section shown in Fig. 3.34c. 

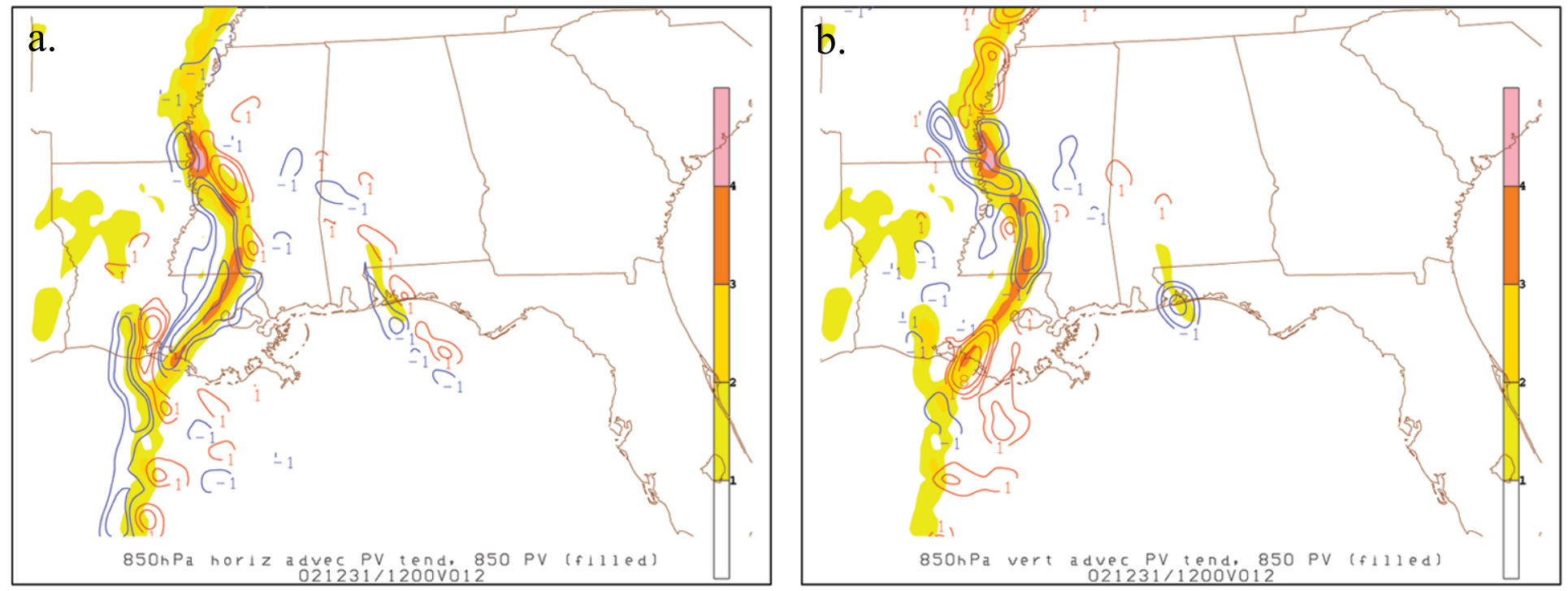

๙
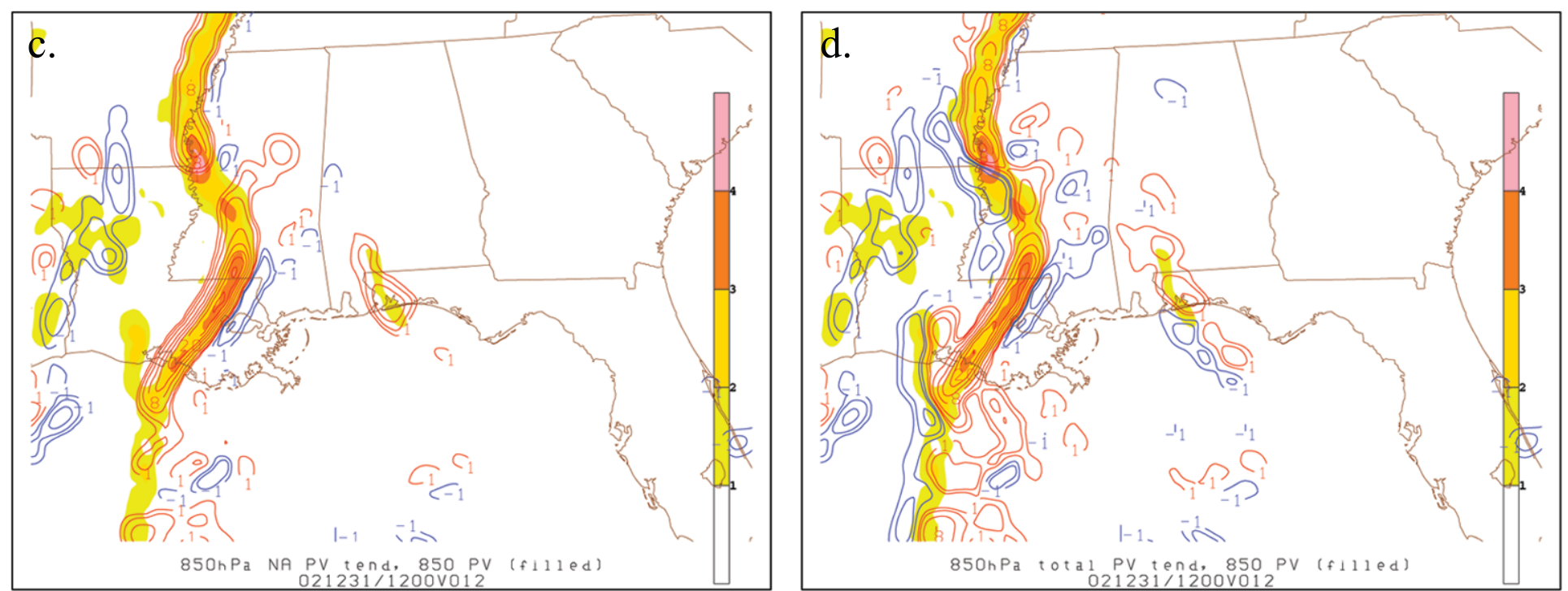

Figure 3.26. PV budget summary for 12 UTC 31 Dec: (a) 800-900-hPa PV (PVU, shaded as in legend at right of panel) and 850-hPa horizontal advective PV tendency (contour interval 1 PVU day-1, red (blue) contours correspond to positive (negative) values); (b) as in (a) except for the vertical advective PV tendency; (c) as in (a) except for nonadvective PV tendency; (d) as in (a) except for total PV tendency. 

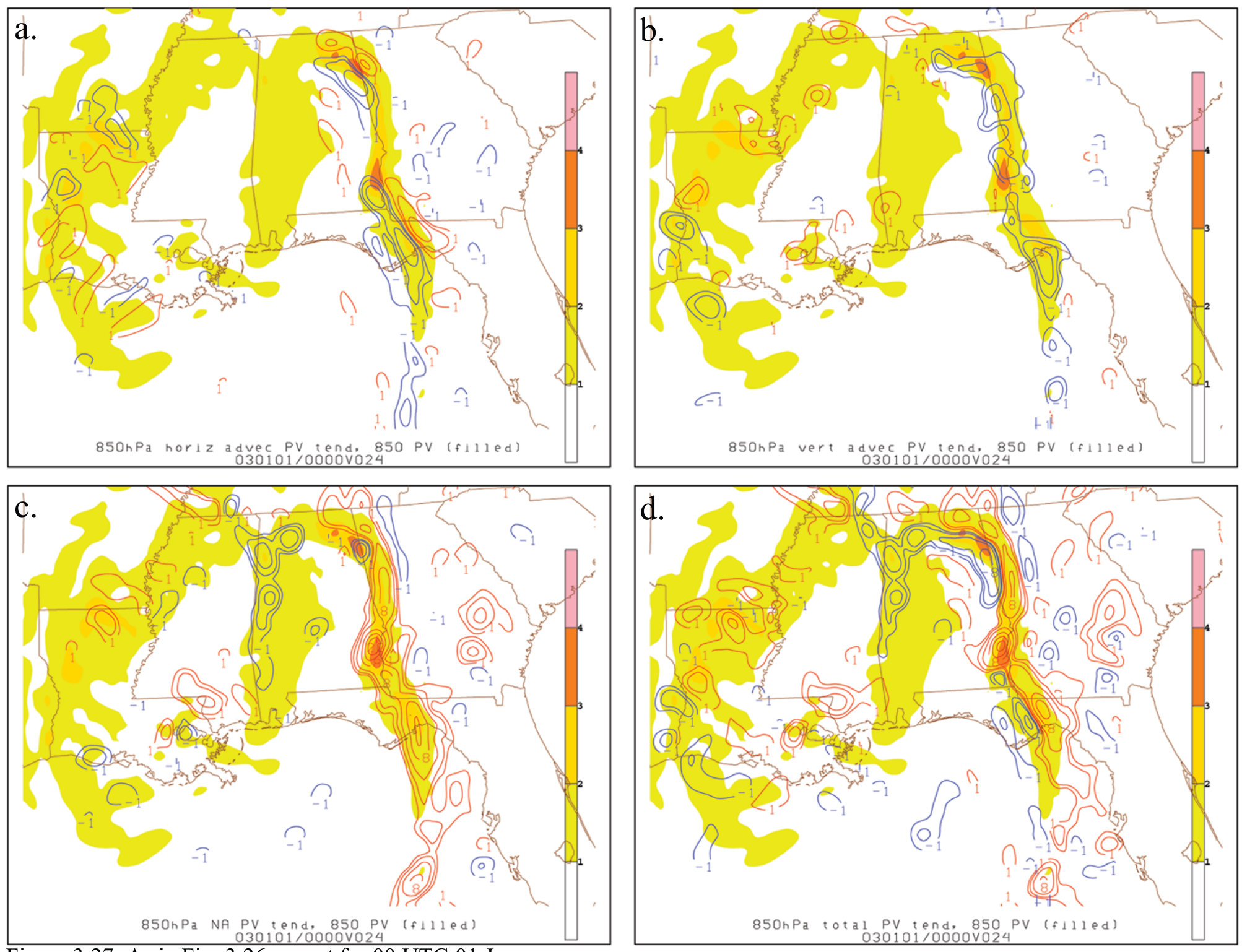

Figure 3.27. As in Fig. 3.26 except for 00 UTC 01 Jan. 


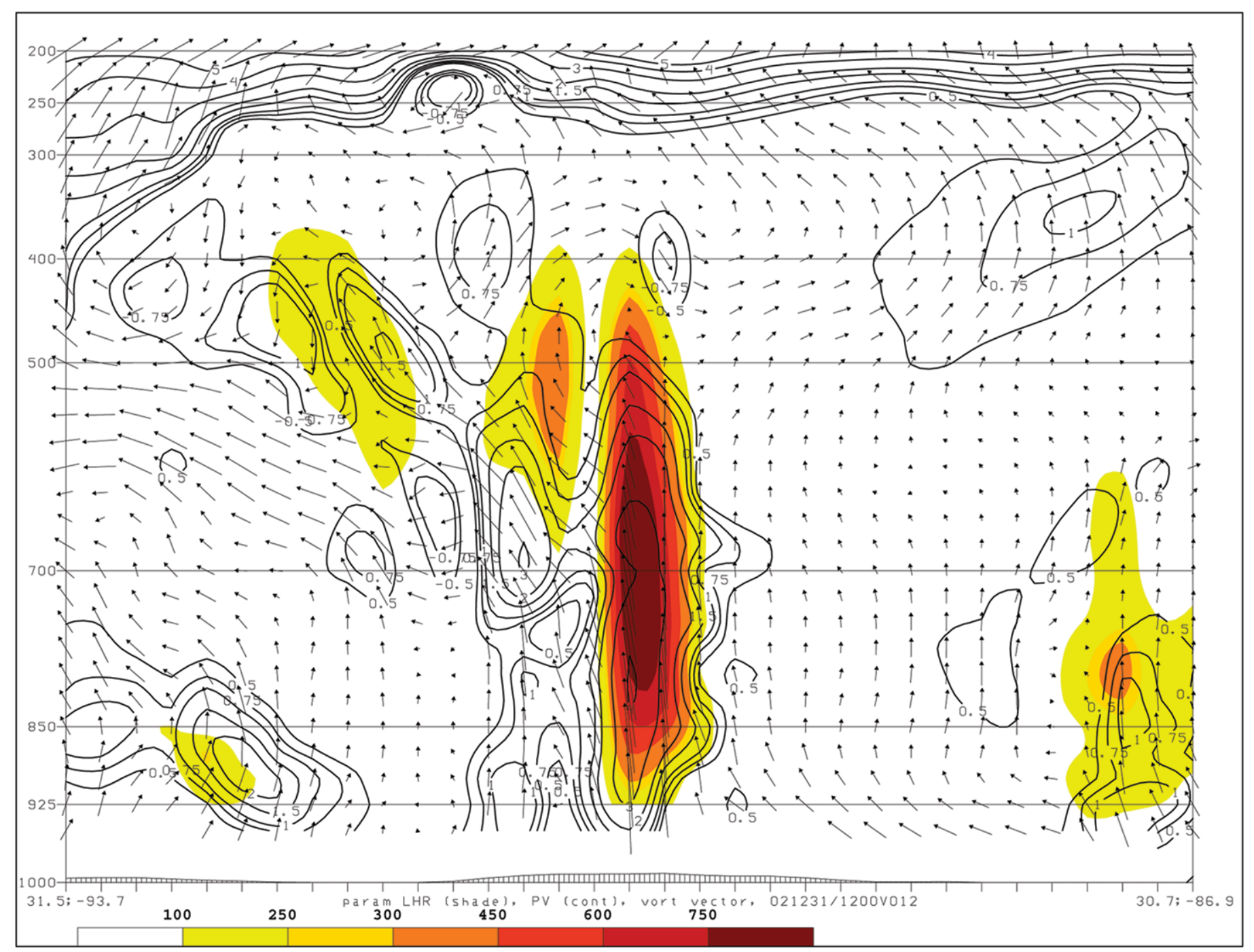

Fig. 3.28. Cross section taken along line A-A' in Fig. 3.25a valid at 12 UTC 31 Dec showing parameterized latent heating (K day-1, shaded as in legend at bottom of panel), PV (solid contours, PVU), and absolute vorticity vectors (arrows). 


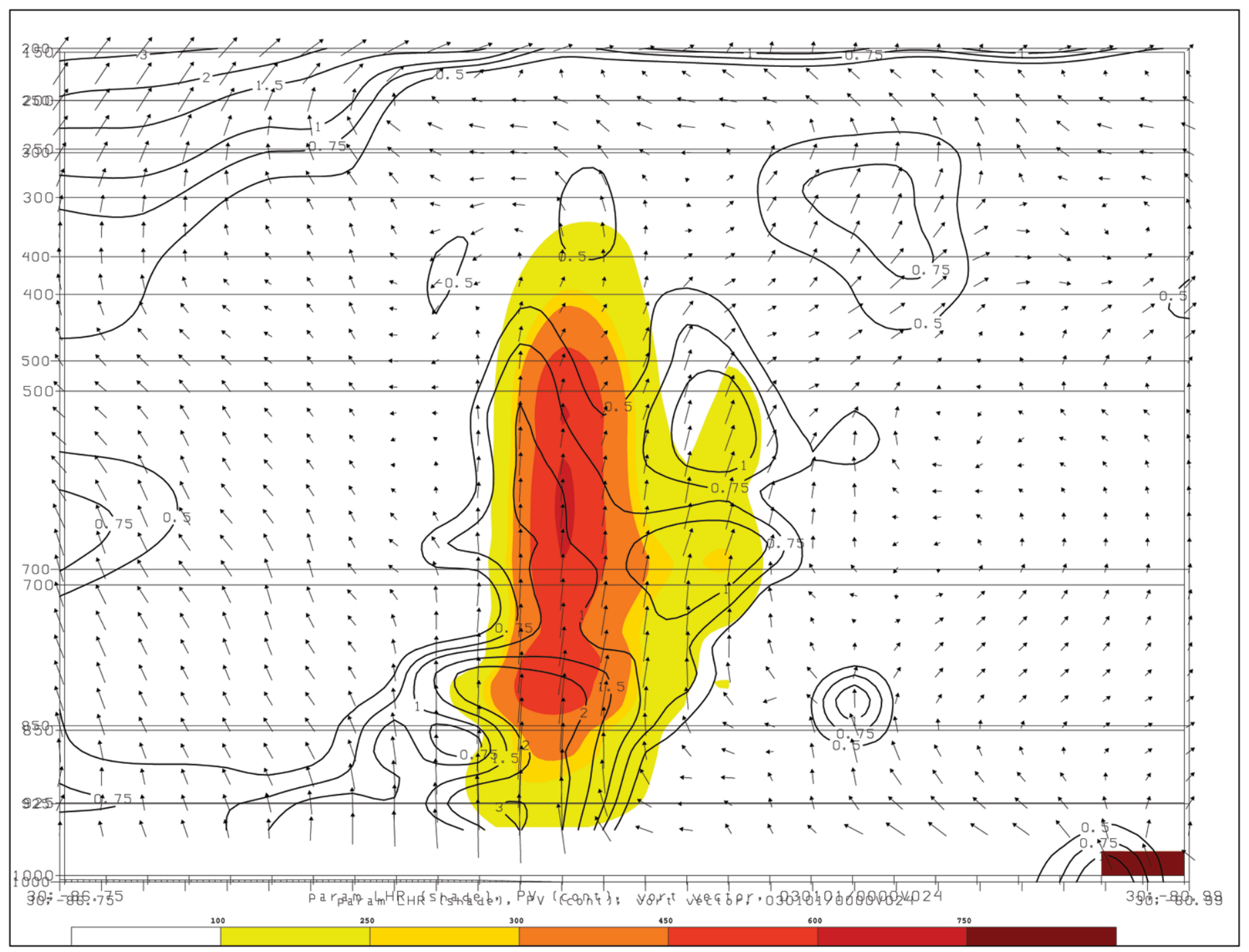

Figure 3.29. As in Fig. 3.28 except cross-section taken along line B-B' in Fig. $3.25 \mathrm{~b}$ and valid at 00 UTC 01 Jan. 


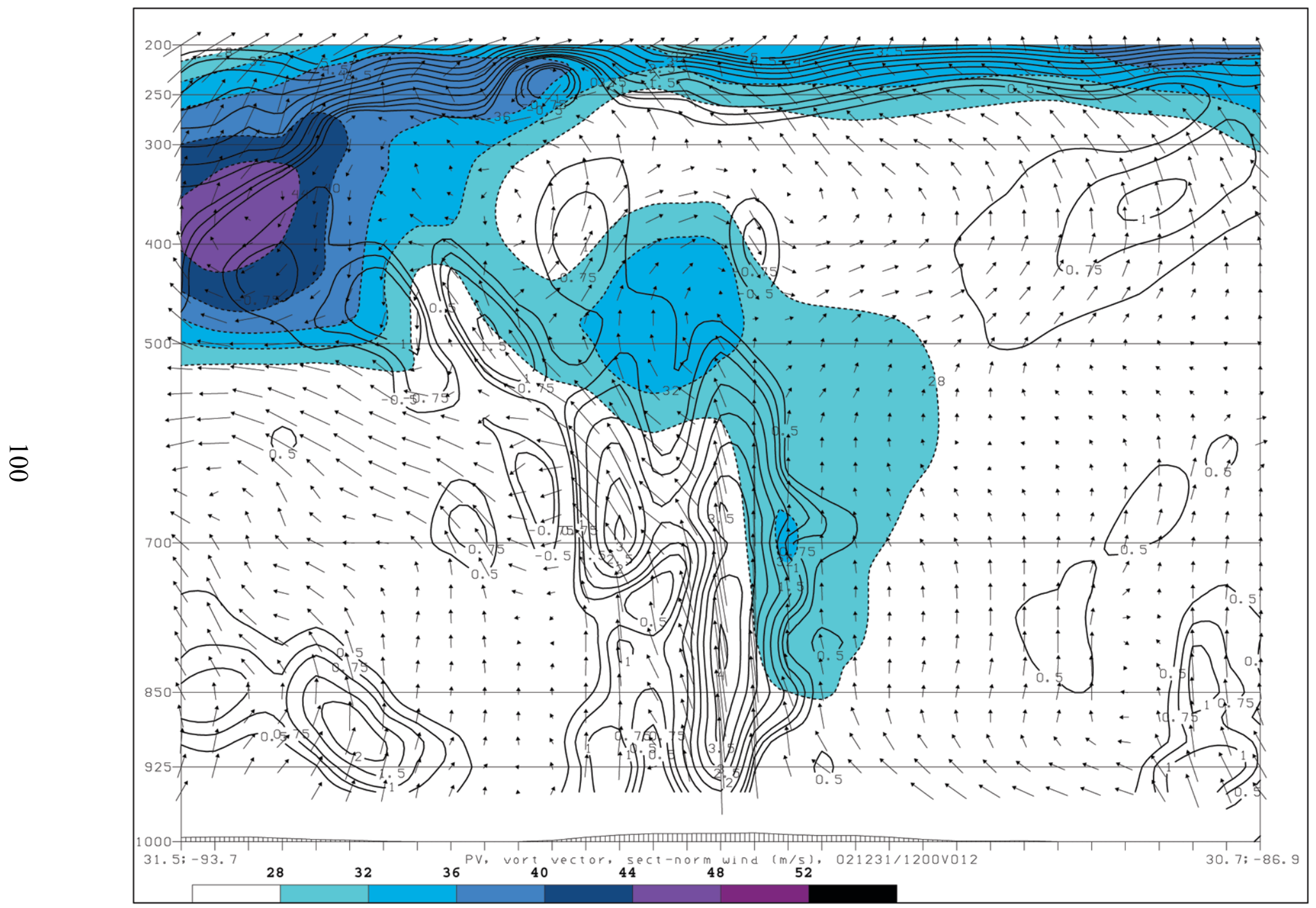

Figure 3.30. Cross section taken along line A-A' in Fig. 3.25a valid at 12 UTC 31 Dec showing PV (solid contours, PVU), absolute vorticity vectors (arrows), and section-normal wind speed (kt, shaded as in legend at bottom of panel) 


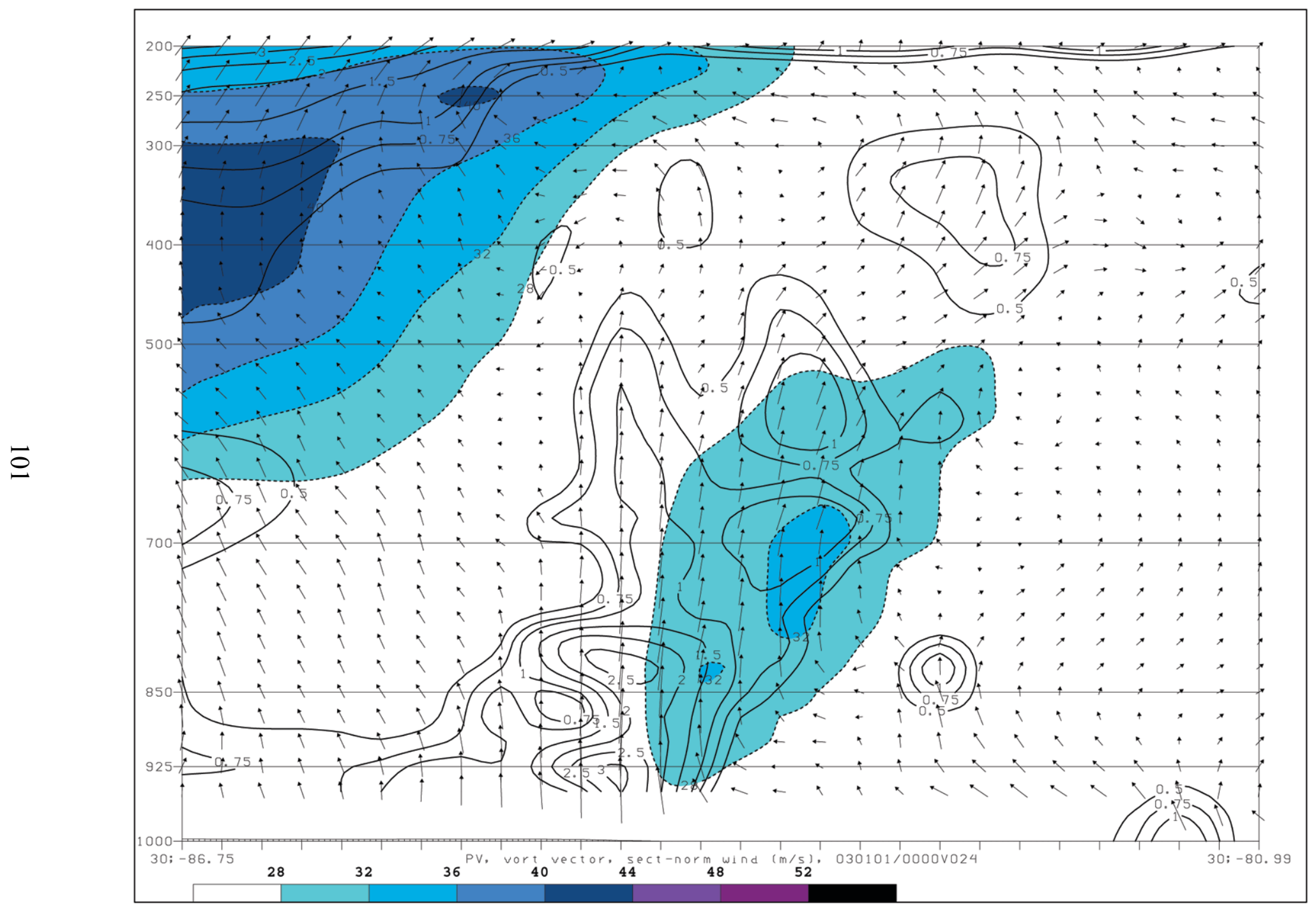

Figure 3.31. As in Fig. 3.30 except cross-section taken along line B-B' in Fig. 3.25b and valid at 00 UTC 01 Jan. 


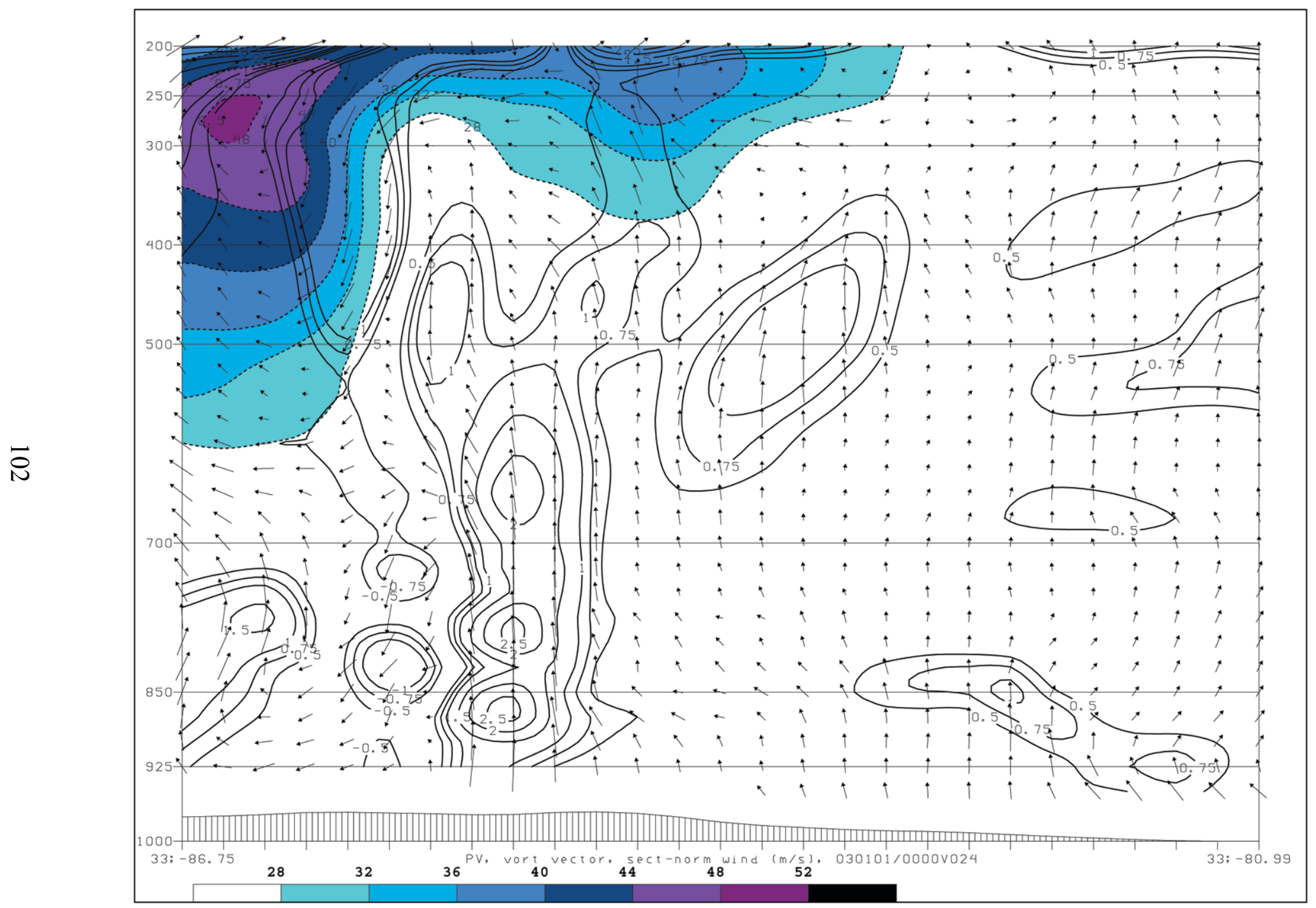

Figure 3.32. As in Fig. 3.31 except cross-section taken along line C-C' 'in Fig. 3.25b. 

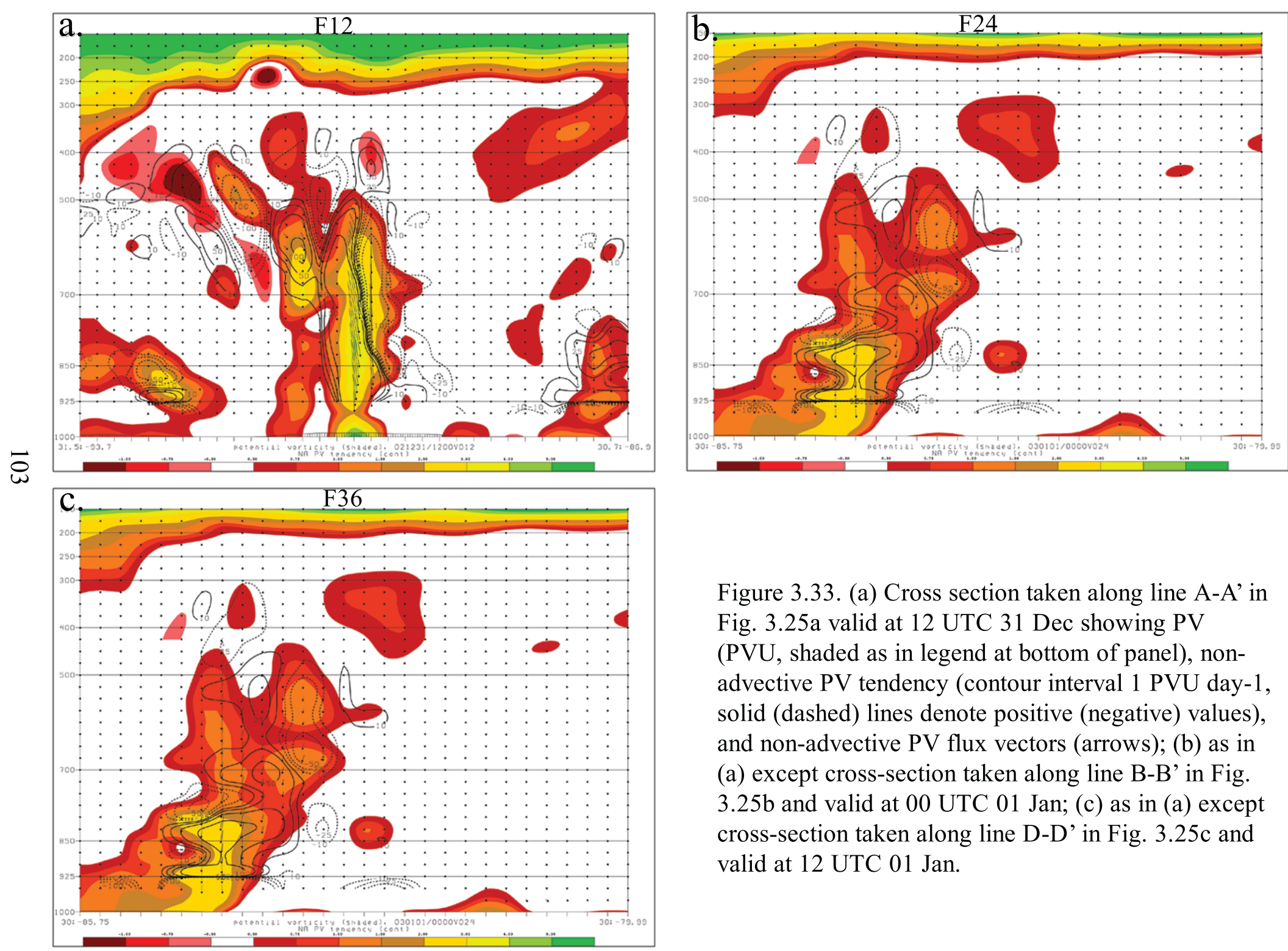

Figure 3.33. (a) Cross section taken along line A-A' in Fig. 3.25a valid at 12 UTC 31 Dec showing PV (PVU, shaded as in legend at bottom of panel), nonadvective PV tendency (contour interval 1 PVU day-1, solid (dashed) lines denote positive (negative) values), and non-advective PV flux vectors (arrows); (b) as in (a) except cross-section taken along line B-B' in Fig. $3.25 \mathrm{~b}$ and valid at $00 \mathrm{UTC} 01 \mathrm{Jan}$; (c) as in (a) except cross-section taken along line D-D' in Fig. 3.25c and valid at 12 UTC 01 Jan. 

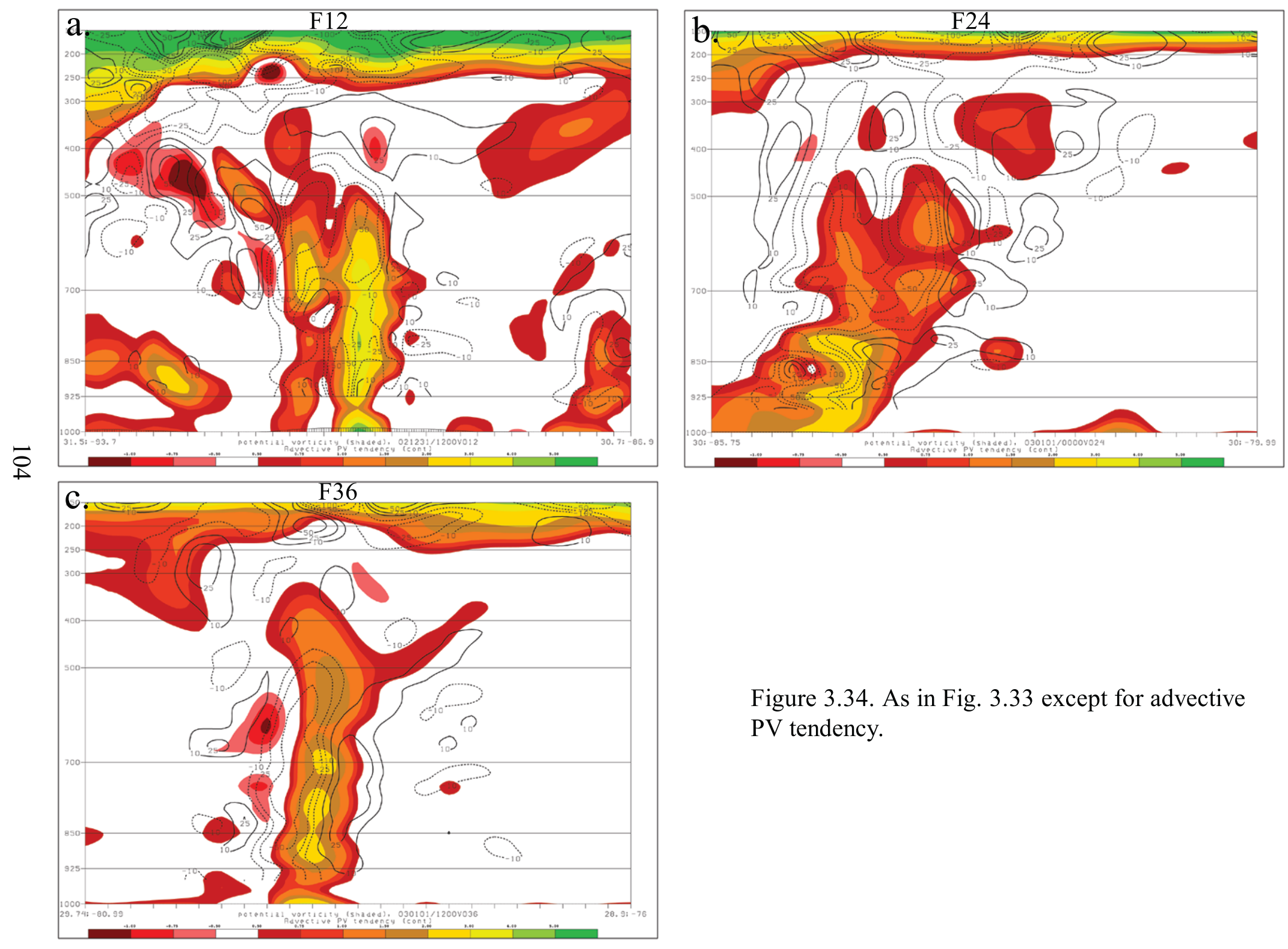

Figure 3.34. As in Fig. 3.33 except for advective PV tendency. 


\section{Scenario 2: 13 - 14 January 2005}

\subsection{Introduction/event summary}

The case chosen to represent Scenario 2 (S2) occurred on 13 - 14 Jan 2005. A slow-moving cold front produced large amounts of precipitation as it passed through the middle and eastern parts of the US (Figs. 4.1, 4.2). Along with high precipitation totals, the system also produced severe weather. 11 tornado reports, 3 hail reports, and 100 high wind reports were recorded by the Storm Prediction Center (SPC) for this event (Fig. $4.3)$.

With respect to QPF, this case can be described as a model QPF "miss" for parts of the Southeast and Mid-Atlantic regions of the US, as precipitation amounts were significantly under-forecasted by operational models at certain times and locations (e.g. Fig. 4.4). The purpose of this analysis is to investigate the source of model error for this case, as well as to examine this case in the context of being a "counter-example" to the S1 case study event, in which UC appears to have decreased downstream precipitation amounts. The distinction is important, as conventional forecast wisdom generally implies that UC systematically decreases the downstream precipitation and that the downstream QPF should thus be reduced in the presence of UC. The analysis of this case will examine the possibility that the opposite may sometimes be true, given a certain type of storm environment. Analyses and operational model forecasts for this case are summarized in the following sections. 


\subsection{Event analysis}

\subsubsection{Observational analysis}

\subsubsection{Synoptic scale analysis}

Figures 4.5 - 4.9 show the Eta 211 analysis fields from 00 UTC 13 Jan to 00 UTC 15 Jan at 12-h intervals. As in Chapter 3, these plots show the evolution of the system at $250 \mathrm{hPa}, 500 \mathrm{hPa}, 850 \mathrm{hPa}$, and the surface. At the 00 UTC $13 \mathrm{Jan}$, a large-amplitude upper-level trough is moving across the Midwest US, evident at both $250 \mathrm{hPa}$ and 500 hPa (Fig. 4.5a,b). Southwesterly low-level flow dominates over the Southeast US at 850 $\mathrm{hPa}$, as well as ahead of the surface cold front (Fig. 4.5c,d). The analysis in Fig. 4.6a, valid 12 UTC 13 Jan, shows that at the $250-\mathrm{hPa}$ level, the maximum winds of the jet stream extend from the Pacific Northwest southward through northern Mexico, and recurve northward toward Lake Superior, lifting toward the northeast. In Fig. 4.6b a shortwave $500-\mathrm{hPa}$ trough is centered over eastern Oklahoma, and southerly flow at 850$\mathrm{hPa}$ is evident over the Southeast US in Fig. 4.6c. At the surface (Fig. 4.6d), a cold front and its associated large area of precipitation extend northeast-southwest from around the western Great Lakes (not shown) to Mississippi, aligning favorably with the right entrance region of the 250-hPa jet stream shown in Fig. 4.6a.

Figure 4.2e shows the location of the convective line at 00 UTC 14 Jan. By this time, the $250-\mathrm{hPa}$ jet core has moved eastward and continues to show signs of lifting, as a strongly confluent pattern aloft becomes evident over the Northeast (Fig 4.7a). At the 500-hPa level, the shortwave noted at 12 UTC 13 Jan has weakened considerably, consistent with the lifting trough (Fig 4.7b), and at the $850-\mathrm{hPa}$ level, the trough has moved eastward, and strong southerly flow is prominent ahead of it (Fig. 4.7c). The band 
of precipitation remains well intact and aligned with the main surface trough, which has likewise moved significantly eastward from 12 UTC 13 Jan (Fig. 4.7d). The alignment of the precipitation relative to the surface cold front in Fig. 4.7d shows the anafront structure that differentiates this case from the more katafrontal type of event examined in the previous chapter.

By 12 UTC 14 Jan (Fig. 4.8), the main jet energy is located to the north and east of the Great Lakes region, extending southward approximately along the Appalachian Mountains (Fig. 4.8a). This configuration places the Southeast US near the right entrance region of the jet, and in a region of large upper-level divergence (Fig. 4.10). At the 500$\mathrm{hPa}$ level the main trough is not as deep as 24 hours prior, and the associated vorticity maximum is much weaker, consistent with a lifting trough structure (Fig. 4.8b). The flow at $850 \mathrm{hPa}$ has switched to a more northerly direction behind the system, but is still southwesterly over the eastern Carolinas, providing moisture to the region along and east

of the front, where precipitation remains heavy (Figs. 4.8c and 4.8d). Figure 4.9 depicts the evolution of the system as it moved off the East Coast, showing that both the upper trough and surface cold front lift off toward the northeast. As described above, Fig. 4.10 elucidates the divergence associated with the right entrance region of the lifting jet steak that appears to be important to the dynamics of this case.

\subsubsection{Mesoscale/Convective scale/Storm scale analysis}

In order to focus more on the system and near-system environment, Figures 4.11 - 4.15 show analyses of parameters and diagnostics illustrative of moisture and instability characteristics in the lower troposphere. At the onset of the event, the system 
environment reveals a moderately strong LLJ and moisture flux signature located along the convective line, oriented northeastward from the Gulf of Mexico (Fig. 4.11). The analysis in Fig 4.12a (valid at 12 UTC 13 Jan) shows moderate instability ahead of the southern portion of the convective line, coupled with significant low-level shear along the frontal zone. Figure 4.12b shows that most lower-tropospheric PV is concentrated in the stable zone behind the surface front, though a region of larger PV does begin to extend into eastern LA at this time. This region of PV may be diabatically-driven, as convection was active in this area at this time. This possibility and its implications will be explored further later in this chapter. The $850-\mathrm{hPa}$ isotachs show that the axis of maximum winds is found along, and just ahead of, the convective line (Fig. 4.12c), and that the LLJ winds are in excess of $75 \mathrm{kts}$ in some localized areas, such as over TN. Moisture flux fields at 12 UTC 13 Jan show two maxima of moisture flux; one just ahead of the southern end of the convective line, and the other to the north, over OH (Fig. 4.12d).

12 hours later at 00 UTC 14 Jan, instability is still present over much of the Southeast US, ahead of the convective line, and the area of maximum shear remains aligned with the surface front (Fig. 4.13a). A low-level PV maximum remains associated with the region of stable air behind the front, but Fig. 4.13b shows that the small area of PV ahead of the front has expanded in size. The maximum values of moisture flux magnitude have also become more concentrated along the southern end of the convective line, particularly over the FL panhandle and central NC/SC (Fig. 4.13d). Observed soundings at this time show moderate instability and strong LLJ winds around the 850$\mathrm{hPa}$ level in the prefrontal air mass (Fig. 4.14). 
By 12 UTC 14 Jan, the convective line has moved farther eastward, and extends along much of the East Coast (Fig. 4.2g). At 12 UTC 14 Jan, most convective instability is concentrated along the Gulf Stream and over the Gulf of Mexico, and low-level shear remains greatest along the surface frontal zone, though a region of lower shear values is evident over SC (Fig. 4.15a). The PV field still shows large PV values in the stable air mass behind the front, but also reveals an area of large PV (likely of diabatic origin) along and ahead of the leading convective edge (Fig. 4.15b). Figure 4.15c shows that by 12 UTC 14 Jan, the core of the 850 -hPa LLJ has moved offshore and is still oriented immediately ahead of the convective line. Finally, moisture flux magnitudes also remain maximized immediately ahead of the convective line, but with a local maximum east of SC, and another along the more northern portion of the line (Fig. 4.15d). As the system moves offshore, moisture flux magnitudes decrease over the Southeast, and a more stable air mass settles in behind the exiting system (Fig. 4.16).

\subsubsection{Analysis of operational forecast}

\subsubsection{Assessment of Eta QPF}

An accurate assessment of the operational model QPF is difficult for this case, as no easily-adaptable gridded analysis dataset exists. (The NARR dataset used in the S1 case study is not yet available for 2005). However, pre-plotted images downloaded from the National Precipitation Verification Unit (NPVU) can be roughly compared to Eta model QPF plots, despite less-than-optimal mapping areas and temporal resolutions. Figure 4.17 shows these NPVU images, which can be roughly compared to the Eta model 
QPF plots in Fig. 4.18. While it is a relatively imprecise method of comparison, several observations can be made.

First, it can be said that at many times, model forecasts and analyses are in general agreement, particularly from 00 UTC 13 Jan - 06 UTC 14 Jan (cf. Figs. 4.17a-f, 4.18a-f). This agreement is a significant difference from the S1 case study, in which an obvious model forecast false alarm was evident. However, the 6-h period ending 12 UTC 14 Jan featured a significant model QPF error (cf. Figs. 4.17g, 4.18g). The Eta model forecast significantly under-predicted precipitation during this period, which is also shown in the observation vs. model forecast comparison in Fig 4.4. This 6-h interval seems to be the crucial period during which to examine the possibility that the UC may have enhanced the downstream precipitation over certain areas for this case.

The operational Eta model forecasts are summarized and compared with Eta model analyses in the following two sections.

\subsubsection{Eta synoptic scale forecast fields}

Figures $4.19-4.22$ show the operational Eta 211 forecasts from the 00 UTC 13 Jan run, valid at 12-h intervals from 12 UTC 13 Jan - 00 UTC 15 Jan. Large-scale differences between the analyses and the forecasts can be seen by comparing Figs. 4.6 4.9 to Figs. $4.19-4.22$, respectively.

By comparing Fig. 4.6a to Fig. 4.19a, it is evident that the 12-h forecast at 250 $\mathrm{hPa}$ under-predicts the maximum winds of the jet stream over Nevada/Arizona/N. Mexico, as well as slightly under-forecasts the winds over the western Great Lakes and southern Ontario. While the $500-\mathrm{hPa}$ and $850-\mathrm{hPa}$ forecast features match analyses to a 
reasonable degree at this time, the surface trough is more progressive in the forecast as compared to the analysis (cf. Figs. 4.19d, Fig. 4.6d).

Comparing the 24-h forecast valid 00 UTC 14 Jan to the analysis fields (cf. Fig. 4.7, Fig. 4.20) shows that one obvious difference at this time was found at the $500-\mathrm{hPa}$ level. Relative to Fig. 4.7b, Fig. 4.20b shows a more elongated region of absolute vorticity throughout the trough axis, while Fig. $4.7 \mathrm{~b}$ shows a more consolidated region of absolute vorticity over southern Illinois. At the $850-\mathrm{hPa}$ and surface level, the forecast matches the analysis reasonably well, with the pressure, temperature and precipitation fields all in overall agreement (cf. Figs 4.7c,d, 4.9c,d).

By 12 UTC 14 Jan, a large forecast/analysis difference is still seen at the $500-\mathrm{hPa}$ level, where the vorticity maximum in the forecast is significantly weaker and located more to the north than observed in the analysis (cf. Figs. $4.8 \mathrm{~b}, 4.21 \mathrm{~b}$ ). The forecast at the 250- and 850-hPa levels appear to be well-matched with analyses, while at the surface, the forecasted low-pressure trough appears to lag slightly toward the west relative to the analysis.

The system has exited the East Coast by 00 UTC 15 Jan, though the forecast is slightly too fast with the eastward movement of the upper trough feature, as the analyzed $250-\mathrm{hPa}$ winds remain stronger over much of the Northeast and Mid-Atlantic US relative to the forecasted values (cf. Figs. 4.9a, 4.22a). Furthermore, the vorticity maximum is still marked at $500-\mathrm{hPa}$ in the analysis at this time, while it is significantly weaker in the 48-h forecast (cf. Figs. 4.9b, 4.22b). At the 850-hPa and surface levels, the forecasted system is located slightly eastward of that in the analyses (cf. Figs. 4.9c,d, 4.22c,d). Further differences will be explored at a smaller scale in the following section. 


\subsubsection{Eta mesoscale forecast fields}

Figs. $4.23-4.26$ compare model forecasts to the analyses using same fields as those plotted in Figs. 4.12 , 4.13, 4.15, and 4.16. While most forecast fields appear to be in general agreement with the analyses, there are several features and model errors that may be of importance. First, the forecast valid at 12 UTC 13 Jan reveals a different lowlevel PV and moisture flux pattern (Figs. 4.23b,d), as the forecast had a more defined PV maximum and a more concentrated region of moisture flux along eastern KY/TN than is observed in the analyses (Figs. 4.12b,d). The LLJ at this time appears to be wellforecasted (cf. Figs. 4.12c, 4.23c).

At 00 UTC 14 Jan, analyses (Fig. 4.13) show stronger shear and instability than was forecast (cf. Figs. 4.13a, 4.24a). Furthermore, the low-level PV signature is also stronger overall and the easternmost edge of the maximum is located slightly farther east than was forecast (cf. Figs. 4.13b, 4.24b). The low-level jet is underpredicted by $5-10 \mathrm{kts}$ over much of NC/SC (cf. Figs. 4.13c, 4.24c) and the moisture flux forecast (Fig. 4.24d) underpredicted the strength of the analyzed moisture flux over central NC/SC (Fig. 4.13d).

By 12 UTC 14 Jan, the system was beginning to move off of the East Coast, and the largest model errors relative to the analyses are found again in the PV and moisture flux fields. The model PV forecast (Fig. 4.25b) did not depict the double-barreled structure evident in the analysis (Fig. 4.15b), and the forecasted moisture flux (Fig. 4.25d) was under-predicted relative to the analysis (Fig. 4.15d). 
Finally, by 00 UTC 15 Jan the system has almost completely moved off of the East Coast, though differences between the model forecast and the analysis persist in the moisture flux fields (cf. Figs. 16d, 26d). The analyzed moisture flux field at this time reveals a much stronger maximum of flux magnitudes over the Atlantic Ocean, though the other fields at this time do appear to be generally well-forecasted.

\subsubsection{Other forecast considerations}

As shown in Fig. 4.3, this storm system was a prolific producer of severe weather. In addition to the tornadoes, hail, and wind damage reported, heavy rainfall also proved to be an important forecasting consideration (Waldstreicher and Figurskey 2005, personal communication).

Beyond the heavy precipitation, hail, and tornadoes, yet another forecast concern was the potential for the strong LLJ winds to mix down to the surface. This was an important consideration in the severe weather forecast process, and the possibility of damaging surface winds became more likely as the LLJ winds increased. While it is beyond the scope of this research, it is worthwhile to note that this issue may be common to S2 events, as similar event from 01 Dec 2004 presented the same threat (Brennan et al., in preparation.) Though this case is not discussed further here, it provides a good example of a system in which strong LLJ winds mixed to the surface to create a severe weather threat in some places, while in other places the strong winds remained safely above the surface stable layer. An important consideration for this case, the diabatic contribution to the strength of the LLJ (discussed in section 4.3) is difficult for models to accurately represent unless the QPF and UC are well forecast. This mixing of the LLJ 
process is beyond the scope of this study, though future research may be undertaken to examine this important problem and its implications for severe weather forecasting.

\subsection{Numerical simulations}

In order to diagnose the diabatic contribution of the convective line to the LLJ, a piecewise QGPV inversion technique was utilized following Lackmann (2002). This exercise will allow a quantitative estimate to be made regarding the degree to which the UC feature may have enhanced the system's associated LLJ, thereby possibly increasing the amount of downstream precipitation. As the operational Eta model did not represent the period of heavy precipitation over the Southeast US for the 6-h period ending 12 UTC 14 Jan, the WRF model was used to obtain a model forecast that better represented this period of precipitation enhancement. The WRF model forecast output used for this procedure is described below.

\subsubsection{WRF control run}

The WRF model forecast used for the PV budget and PV inversion is described in detail in Chapter 2. The following sections assess the WRF model forecast and compare it to the observations and analyses described in the preceding sections. Section 4.4 will examine the results of the PV diagnostics and will discuss their implications for this case. 


\subsubsection{Assessment of WRF control run}

In order to compare the WRF forecast for the 13-14 Jan 2005 event to analyses, 4-panel plots analogous to those previously discussed are presented. QPF, as well as synoptic and mesoscale forecast fields are evaluated and discussed below.

\subsubsection{Assessment of WRF QPF}

Figure 4.27 shows the 6-h QPF from the WRF forecast, which can be (somewhat awkwardly) compared to corresponding panels in Fig. 4.17 and 4.18, which show the NPVU precipitation analyses and Eta QPF, respectively. Though the NPVU analyses are rather coarse (32-km gridspacing), it can be seen that overall, the WRF precipitation forecast is adequate with respect to time, amount, and location. This remains true even during the 12 UTC 14 Jan time period in which the operational Eta QPF was relatively poor (cf. Figs. 4.17g, 4.18g, 4.27g). Furthermore, comparison of the WRF QPF fields to radar observations (cf. Figs. 4.2, 4.27) shows that the movement and the location of the leading convective line of the UC feature as represented by the WRF forecast and as observed by radar are in close agreement.

Perhaps a more revealing comparison can be made by comparing the WRF QPF plots to those of the operational Eta model (cf. Figs. 4.18, 4.27). Many of the most obvious differences between Fig. 4.27 and Fig. 4.18 can be attributed to the difference in resolution between the operational Eta model forecast ( $\sim 80 \mathrm{~km}$ projection) and the WRF forecast $(20 \mathrm{~km})$, however, there are some notable differences in QPF between the two

runs. In particular, it can again be noted that the WRF QPF appears to be considerably 
more accurate at the 12 UTC 14 Jan time, better representing the area of heavy precipitation over central NC/SC relative to the Eta QPF (Fig. 4.28).

\subsubsection{WRF synoptic forecast features}

Figures 4.29 - 4.31 show the WRF forecasts at 00 UTC 14 Jan (F012), 12 UTC 14 Jan (F024), and 00 UTC 15 Jan (F036), respectively. (Eta model analyses valid at these same times are shown in Figs. 4.7 - 4.9.)

It should again be noted that the difference in resolution of the Eta analyses used here and WRF forecasts causes the character of most forecast features to appear quite different, even if they are showing essentially the same forecast signal. Aside from these resolution-based differences, there is some variation between the runs that appears to be significant. First, at 00 UTC 14 Jan, the forecasted western branch of the jet stream (Fig. 4.29a) is about $25 \mathrm{kt}$ too weak as compared to the analysis (Fig. 4.7a).

At 12 UTC 14 Jan, the jet stream is beginning to lift along the East Coast, placing much of the Southeast US in a region of significant upper-divergence (cf. Figs. 4.8a, 4.30a). The representation of this upper-divergence in the WRF and operational Eta forecasts is compared to the Eta analysis in Fig. 4.32. The Eta model clearly underpredicts this feature over NC/SC, while the WRF divergence field may be slightly overforecasted (though the true difference is ambiguous due to the differing resolutions). The overall spatial distribution of the WRF upper-divergence forecast is superior to that of the operational Eta forecast (particularly with respect to the area of divergence over the central and eastern Carolinas) at this time. Furthermore, at 12 UTC 14 Jan, the magnitude of the vorticity maximum at $500 \mathrm{hPa}$ is slightly overforecasted (cf. Figs. $4.8 \mathrm{~b}, 4.30 \mathrm{~b}$ ) by 
the WRF run, though the significance of this could again be arguably ascribed to resolution issues. At this time the amplitude of the $850-\mathrm{hPa}$ trough is also overforecasted by the WRF (cf. Figs. 4.8c, 4.30c).

Perhaps the most important feature at 00 UTC 15 Jan is associated with the 500$\mathrm{hPa}$ shortwave and associated vorticity maximum (cf. Figs. 4.9b, 4.31b). Other than the character of the feature being different (likely due to resolution differences), the WRF forecast is fairly accurate, compared with the erroneous operational forecast at this time, in which the vorticity maximum is not as strong (Fig. 4.33). It is possible that this weaker feature in the operational forecast may have played a role in the poor operational QPF around this time.

\subsubsection{WRF mesoscale forecast features}

Figures $4.34-4.36$ show WRF forecasts of the mesoscale-type quantities (of which analogous analyses are plotted in Figs. 4.13, 4.15, and 4.16). It should be noted, though, that the two sets of plots are not directly comparable for several reasons. First, as noted above, the different grid spacing of the operational Eta analyses/forecasts and WRF forecasts causes the character of certain features to appear quite different, even if the essence of the forecast is the same. Due to resolution issues with mesoscale features in particular, the $80-\mathrm{km}$ Eta analysis shown here is likely not the best source for verification

of the WRF forecast. Second, the WRF output does not extrapolate winds below the surface, therefore there are regions over the Appalachian Mountains where no shear values are plotted. 
These minor issues aside, there are some important considerations that result from a comparison of the WRF forecasts to Eta analyses. At 00 UTC 14 Jan, the WRF forecast (Fig. 4.34) appears quite different from the Eta analysis (Fig. 4.13). One stark distinction between the two figures can be seen in the low-level PV field (cf. Figs. 4.13b, 4.34b), as the WRF forecast resolves a thin line of high PV air associated with the leading edge of the convective line. Such a representation is missing, or at best greatly smoothed over, in the Eta analysis. Another significant difference between the two plots is that the WRF moisture flux forecast appears to be under-predicted along the FL panhandle and central $\mathrm{NC} / \mathrm{SC}$, relative to the analysis (cf. Figs. 4.13d, 4.34d).

At 12 UTC 14 Jan, the WRF forecast of the low-level PV maintains its distinct leading band of PV (Fig. 4.35b). The LLJ is under-forecasted by the WRF model in the area off of the Mid-Atlantic coast in the WRF run (cf. Figs. 4.15c, 4.35c), though it is still stronger than in the operational Eta run (not shown). At this time, the WRF moisture flux forecast appears to be relatively accurate compared to the analysis (cf. Figs. 4.15d, $4.35 d)$.

By 00 UTC 15 Jan, the WRF forecast remains in good agreement overall with the analysis as the system moves off the East Coast (cf. Figs. 4.16, 4.36). Slight differences include stronger flow at the $850-\mathrm{hPa}$ level than that which was analyzed, and the moisture flux magnitudes over the Mid-Atlantic are slightly too low in the forecast relative to the analyses (cf. Figs. 4.16c,d, 4.36c,d). 


\subsection{PV investigation}

PV is a useful tool with which to investigate the possibility of the convectivelydriven diabatic enhancement of the LLJ, due to its advantageous properties of conservation and invertibility (e.g., Davis and Emanuel 1991). As discussed in Chapter 2, the goal of using PV to investigate this event is to quantitatively measure the effects of diabatic processes on the enhancement of the LLJ. An enhanced LLJ could in turn increase moisture transport ahead of the system, and thereby increase downstream precipitation amounts.

PV budgets are performed so that certain areas of PV may be attributed to particular processes or model physics scheme, while an inversion technique is used to quantify the impact of these PV "parts" (e.g. Stoelinga 1996). PV inversion is a convenient means by which the impact of diabatic processes on surrounding fields may be unambiguously identified and quantified. Specifically, a QGPV inversion is performed here to quantify the effect of diabatic heating on the strength of the LLJ and associated moisture transport.

The methodology used here is described in Chapter 2, and is similar to that used in Lackmann (2002).

\subsubsection{Ertel PV budget}

In order to identify the contribution of LHR to the low-level PV feature and its associated LLJ, a PV budget was performed on the WRF model output. Figure 4.37 shows the 800-900 hPa PV and LHR configuration at 12 UTC 13 Jan, 00 UTC 14 Jan, 12 UTC 14 Jan, and 00 UTC 15 Jan. The leading band of large PV is a result of the 
convective line, which was present over the area at this time (Fig. 4.2), while the PV evident to the west of the leading line marks the high stability of the post-frontal air mass. Given the placement of the band of large PV and LHR maximum, forecast hours 12 and 24 (00 UTC 14 Jan and12 UTC 14 Jan, respectively) were chosen for further investigation.

Figures 4.38 and 4.39 show the $850-\mathrm{hPa}$ total PV budgets for F12 and F24, respectively. Figure 4.38 shows that while horizontal and vertical advective fluxes are active (panels a,b), the nonadvective PV flux (i.e. that created by LHR) (panel c) is the dominant player in the overall positive PV tendency (panel d) at the 850-hPa level at this time. Likewise in Fig. 4.39, the nonadvective PV flux associated with LHR provides the main contribution to the total PV tendency at this time.

In order to provide perspective on the vertical structure of the PV tendency, Figs. 4.40 and 4.41 show cross-sections through the UC diabatic PV maximum (taken through the lines plotted in Figs. 4.37b,c) that illustrate the relationship of the LHR to the PV, with positive PV tendencies generated just below the maximum level of LHR (as would be expected from the PV tendency equation discussed in Chapter 2). Figures 4.42 and 4.43 show the association of section-normal wind speeds to the PV feature at F12 and F24, respectively. From these images, it is clear that the LLJ ahead of the system is indeed closely associated with the low-level PV maximum. The effect of the low-level maximum on the surrounding wind field will be quantified by the PV inversion discussed in the following section.

In terms of PV tendency, the PV budget yields some insight into why this system propagated so slowly (relative to a $\mathrm{S} 1$ event). As discussed in Chapter 2, the 
nonadvective PV tendency is directed opposite the absolute vorticity vector, and thus areas of positive PV tendency are found nearly directly below and to the rear of the system, following the negative of the absolute vorticity vectors (plotted in Figs. 4.40 and 4.41). Figures 4.44 and 4.45 show both the nonadvective and advective PV tendencies, from which any signal of forward propagation due to the presence of LHR or advection is weak, as seen by the lack of significant positive PV tendencies ahead of the system. In order to quantify the effect of LHR on the strength of the LLJ, a PV inversion was performed and is discussed in the following section.

\subsubsection{QGPV inversion}

\subsubsection{The use of QGPV}

As discussed in Chapter 2, the disadvantage of using QGPV relative to Ertel PV is a loss of accuracy in strongly curved flows, while the advantage is that it eliminates the ambiguity that accompanies the inversion of Ertel PV (Davis 1992b). The use of QGPV is appropriate here due to the relatively straight nature of the LLJ feature, although errors on the order of $10 \%-20 \%$ are expected (Hakim et al. 1996, section 3a). Because the goal of this procedure is to estimate the contribution of the low-level PV maximum to the strength of the LLJ and the associated moisture transport, this margin of error is acceptable. Further justification of the use of QGPV is found by subjectively comparing the QGPV and Ertel PV in the lower troposphere (e.g. Fig 4.46), confirming that the spatial distributions of the two quantities are sufficiently similar. 


\subsubsection{Results of the QGPV inversion}

The results of the QGPV inversion performed for 00 UTC 14 Jan show that a cyclonic signal is found in the corresponding height and wind fields, indicating that the band of cyclonic PV associated with the diabatic PV anomaly had a considerable impact on its surrounding environment. Figure 4.47 a shows the lower-tropospheric diabatic PV anomaly associated with the UC is effectively isolated by this method of inversion. Figure $4.47 \mathrm{~b}$ shows the inverted height and wind fields that are a result of the QGPV shown in panel (a). The large height anomaly and associated flow shows that the inverted QGPV feature induces a considerable deepening of the low pressure system (of up to 5 $\mathrm{hPa}$ in the downstream region), as well as significant southerly flow ahead of the system (exceeding $35 \mathrm{kt}$ in the downstream region). The cross-section in Fig. 4.47c shows that southeasterly flow in excess of $14 \mathrm{~m} / \mathrm{s}$ is associated with this feature. For the purpose of comparison, Fig. 4.47d shows the Ertel PV and the section-normal geostrophic flow. While not in perfect agreement, the QGPV in Fig. 4.47a does adequately match the EPV in Fig. 4.47d. Figure 4.48 shows the same fields for a cross-section taken further to the south. Finally, Fig. 4.49 shows the ratio of the inverted geostrophic wind speed to the total geostrophic wind speed. Values exceeding $40 \%$ in the downstream area show that the UC feature clearly contributed strongly to the southerly LLJ east of the system. The findings from the QGPV inversion indicate that the flow associated with the diabatic PV feature produced by the UC significantly contributed to the low-level southerly flow that helped provide ample moisture to the downstream region.

The results from the S2 case study are valuable, as this case demonstrates that the presence of UC actually may increase the amount of moisture that penetrates the 
downstream area. This is an important difference from S1, in which the presence of UC may act to decrease the availability of moisture and amount of precipitation in the downstream area. For this case, however, the PV inversion shows that the UC acts to diabatically enhance the LLJ (via mechanism (iii)), which in turn acts to increase the amount of downstream precipitation. Therefore, the results prove that the presence of UC does not unequivocally reduce downstream moisture and downstream precipitation, as was perhaps previously thought. 


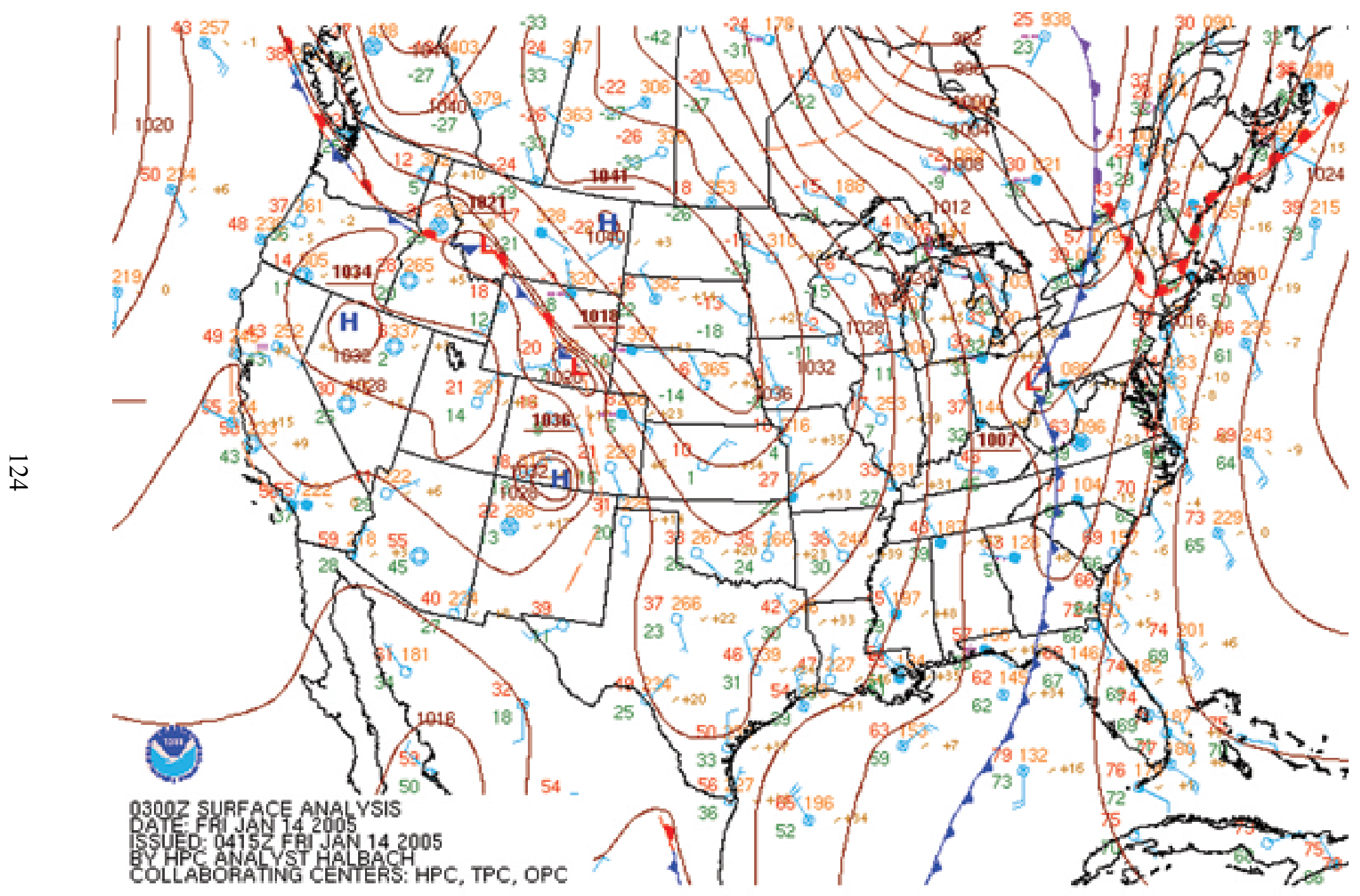

Figure 4.1. Surface analysis and surface observations from Hydrometeorological Prediction Center (HPC), valid 0415 UTC 14 Jan 2005, using conventional symbols and notation. 

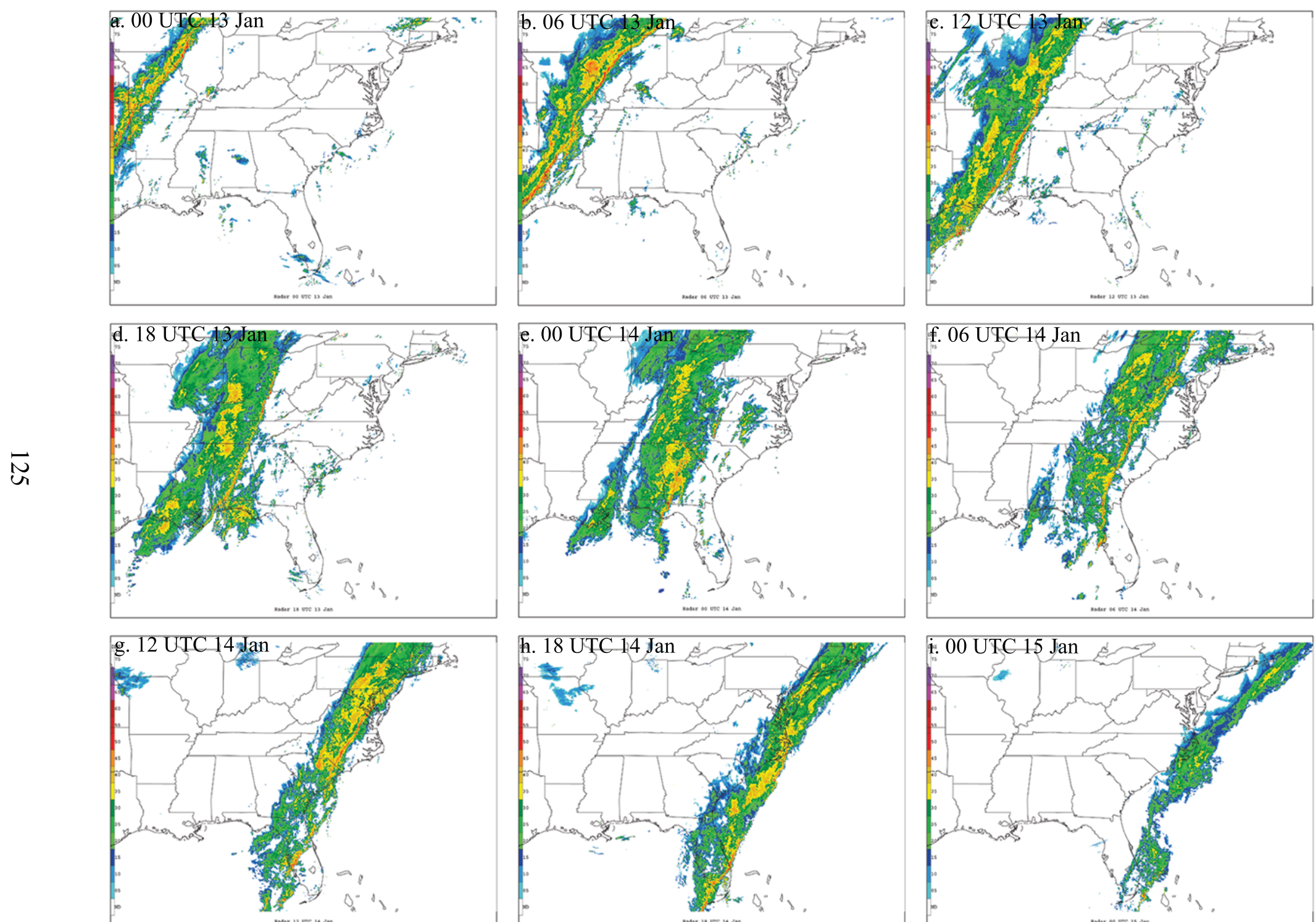

Figure 4.2. Radar mosaic imagery valid at: (a) 00 UTC 13 Jan; (b) 06 UTC 13 Jan; (c) 12 UTC 13 Jan; (d) 18 UTC 13 Jan; (e) 00 UTC 14 Jan; (f) 06 UTC 14 Jan; (g) 12 UTC 14 Jan; (h) 18 UTC 14 Jan; (i) 00 UTC 15 Jan.observations from Hydrometeorological Prediction Center (HPC), valid 0415 UTC 14 Jan 2005, using conventional symbols and notation. 


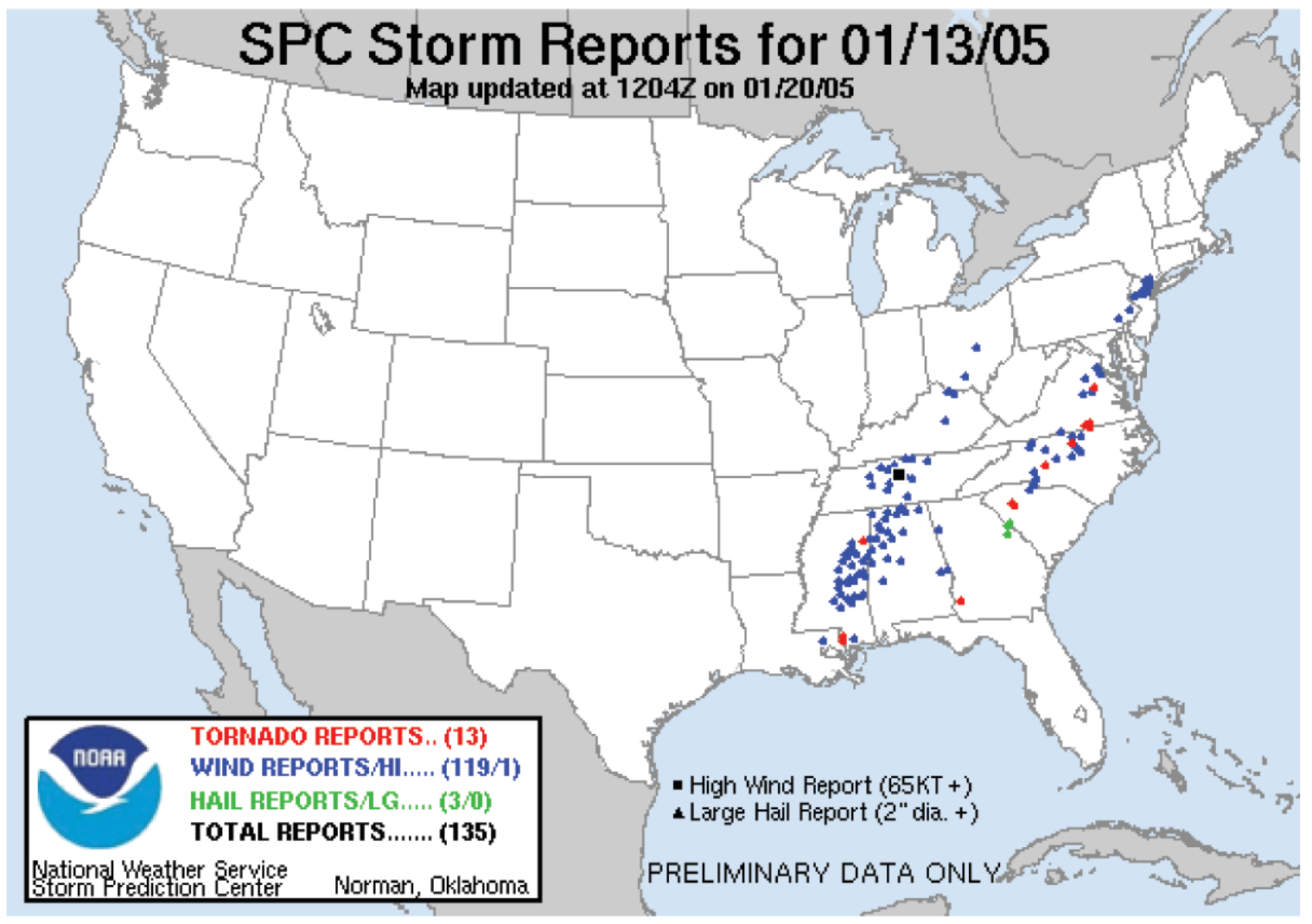

Figure 4.3. Severe weather reports as reported by the Storm Prediction Center for 13 Jan 2005. 


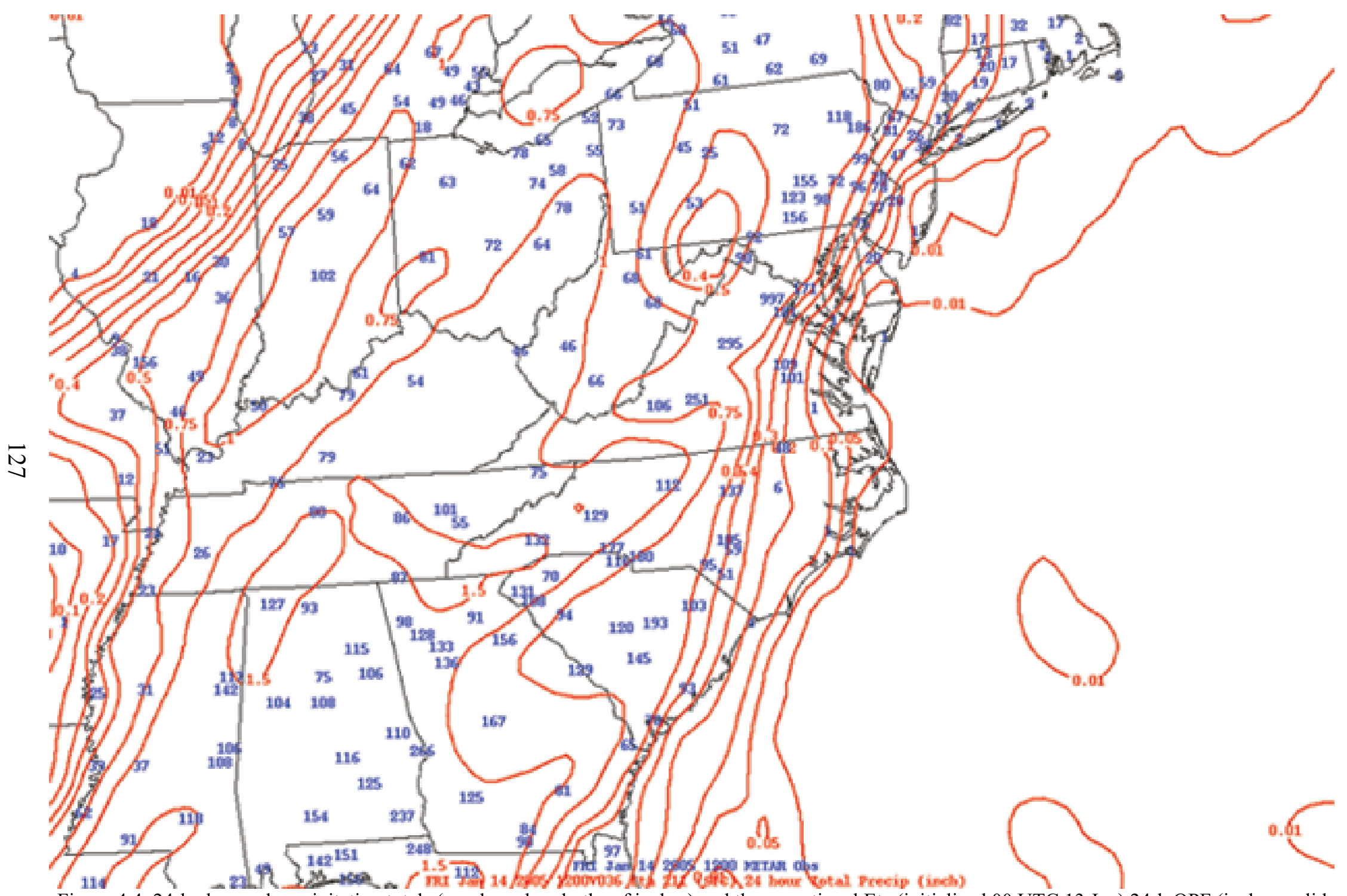

Figure 4.4. 24-h observed precipitation totals (numbers, hundreths of inches) and the operational Eta (initialized 00 UTC 13 Jan) 24-h QPF (inches, solid contours), valid 12 UTC 14 Jan. 

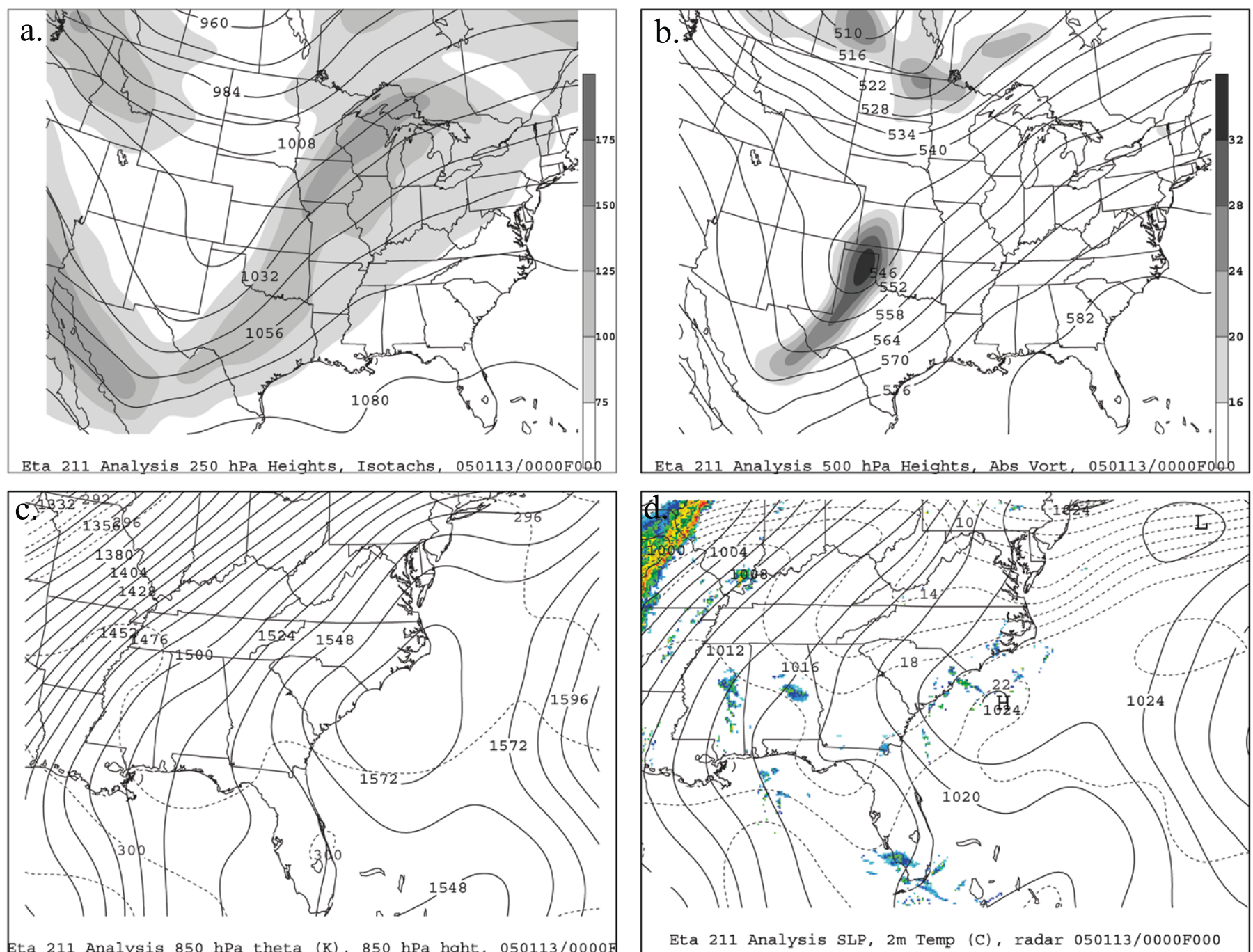

Figure 4.5. Eta Data Assimilation System (EDAS) analysis synoptic summary for 00 UTC 13 Jan 2005: (a) 250-hPa Geopotential height (solid contours, interval 12 dam) and isotachs (kt, shaded as in legend at right of panel); (b) 500-hPa Geopotential height (solid, interval 6 dam) and vorticity ( $10^{\wedge}-5 \mathrm{~s}^{\wedge}-1$, shaded as in legend at right of panel); (c) 850 -hPa potential temperature (dashed contours, interval $2 \mathrm{~K}$ ), $850-\mathrm{hPa}$ Geopotential height (solid, interval 6 dam), and $850-\mathrm{hPa}$ mixing ratio (shaded, interval $2 \mathrm{~g} / \mathrm{kg}$, values below $6 \mathrm{~g} / \mathrm{kg}$ omitted); (d) sea level pressure (solid contours, interval $2 \mathrm{hPa}$ ), 2 -m temperature (dashed contours, interval $2 \mathrm{C}$ ), and mosaic radar imagery (dBz, shaded as in legend at left of panel). 

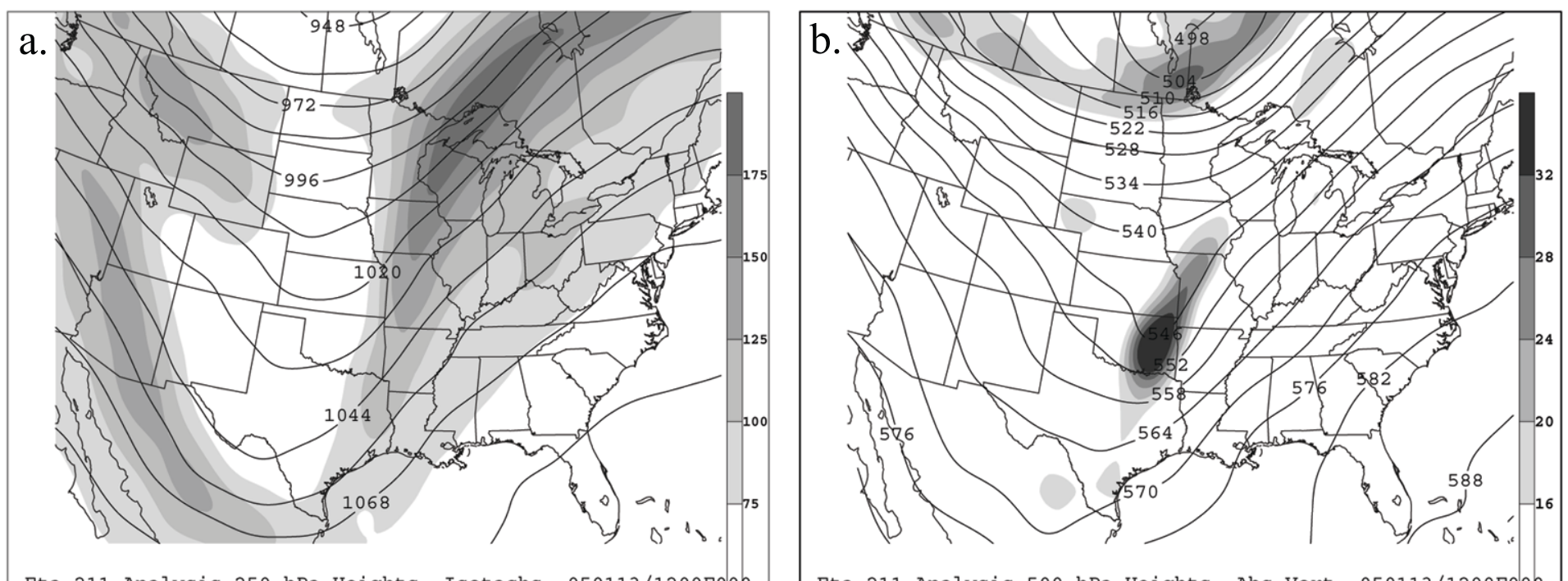

స్

Eta 211 Analysis $250 \mathrm{hPa}$ Heights, Isotachs, 050113/1200F000

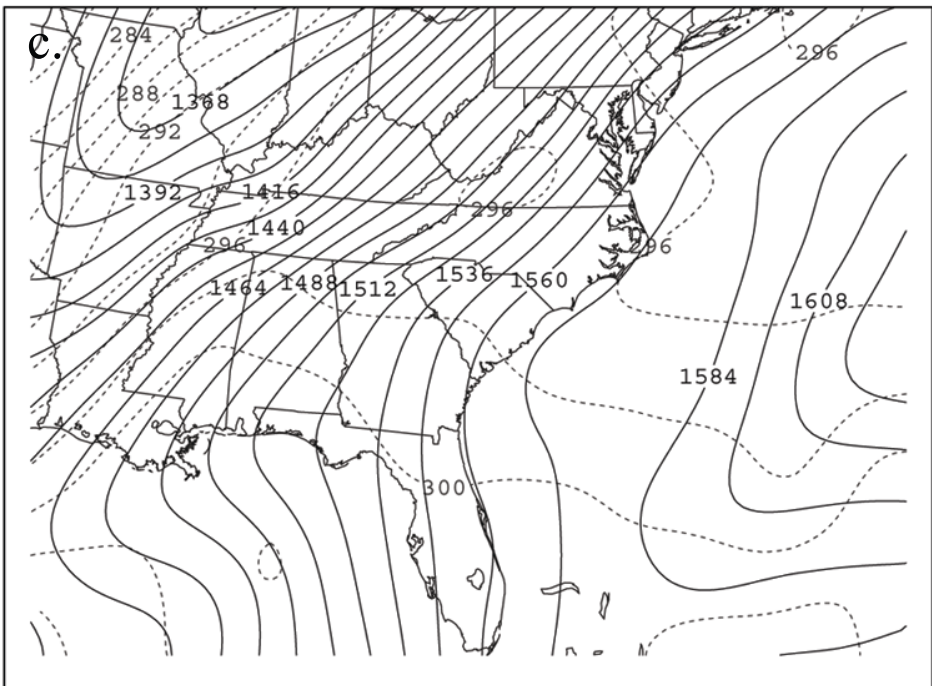

Eta 211 Analysis $850 \mathrm{hPa}$ theta (K), $850 \mathrm{hPa}$ hght, $050113 / 1200$

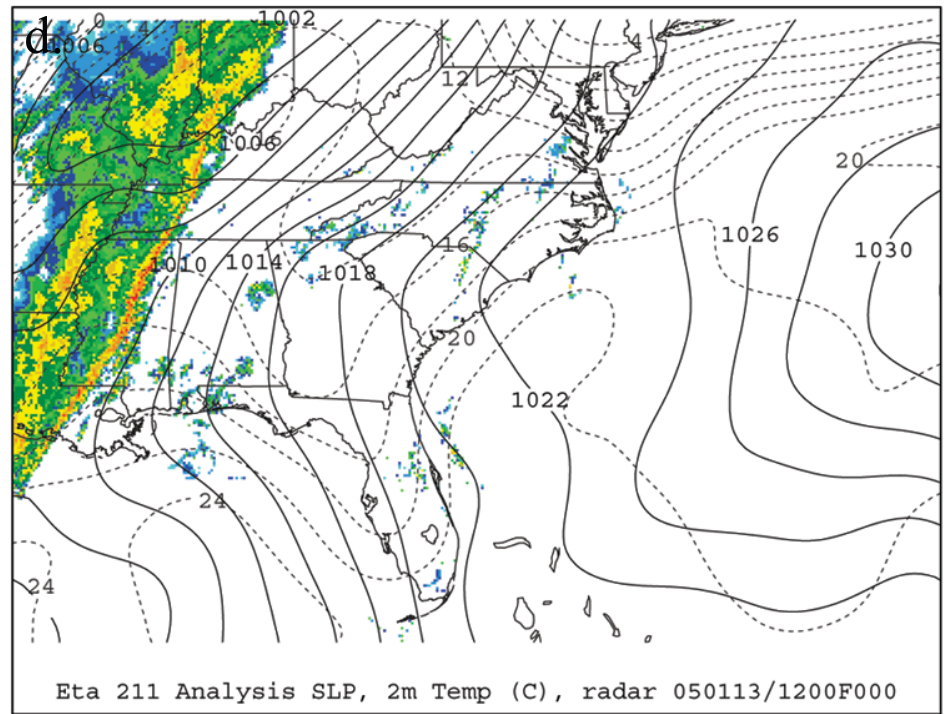

Figure 4.6. As in Fig. 4.5 except for 12 UTC 13 Jan. 

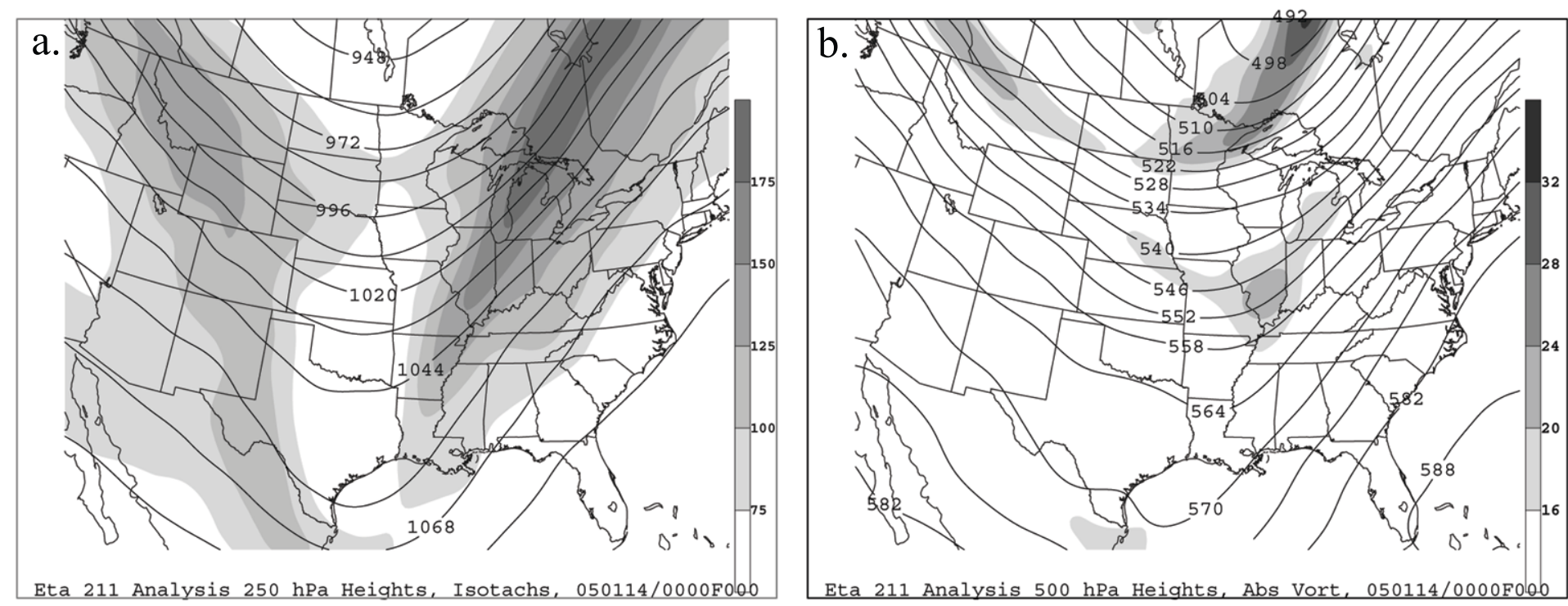

$\overline{\check{o}}$

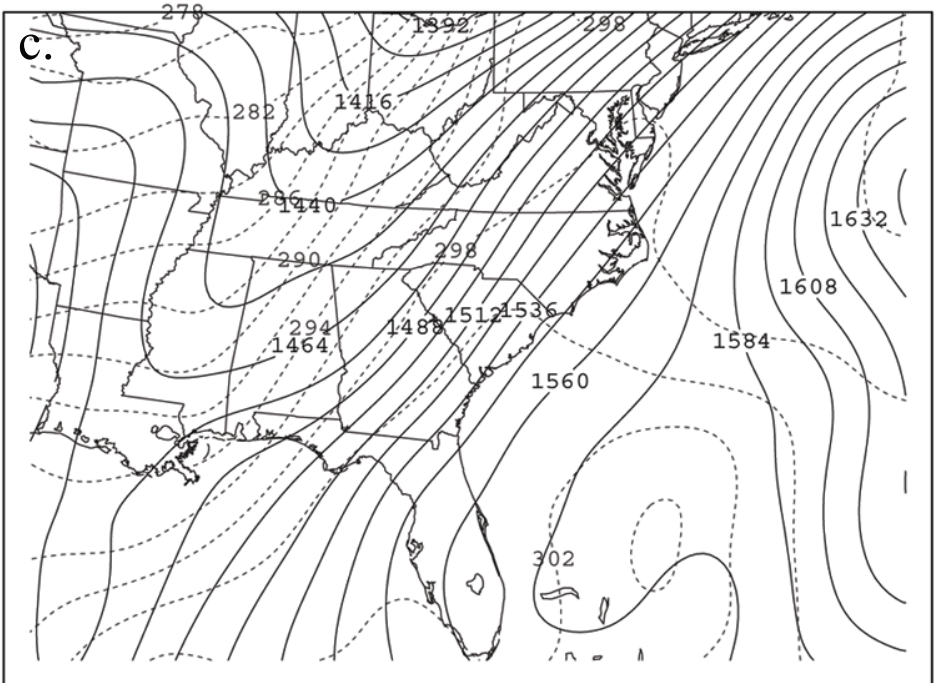

Eta 211 Analysis $850 \mathrm{hPa}$ theta (K), $850 \mathrm{hPa}$ hght, $050114 / 0000 \mathrm{H}$ Figure 4.7. As in Fig. 4.5 except for 00 UTC 14 Jan.

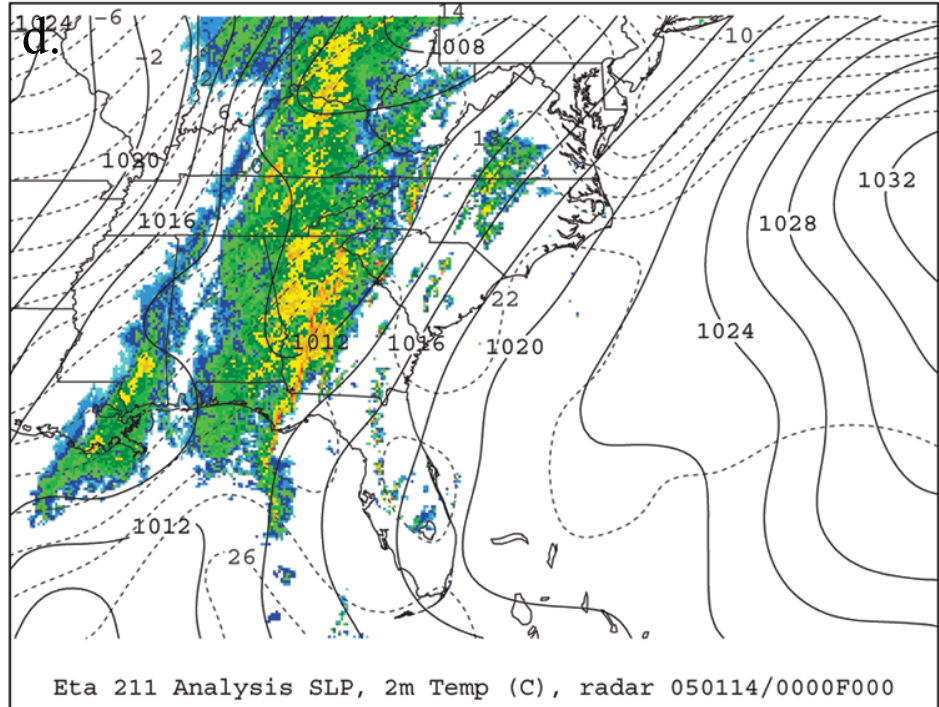



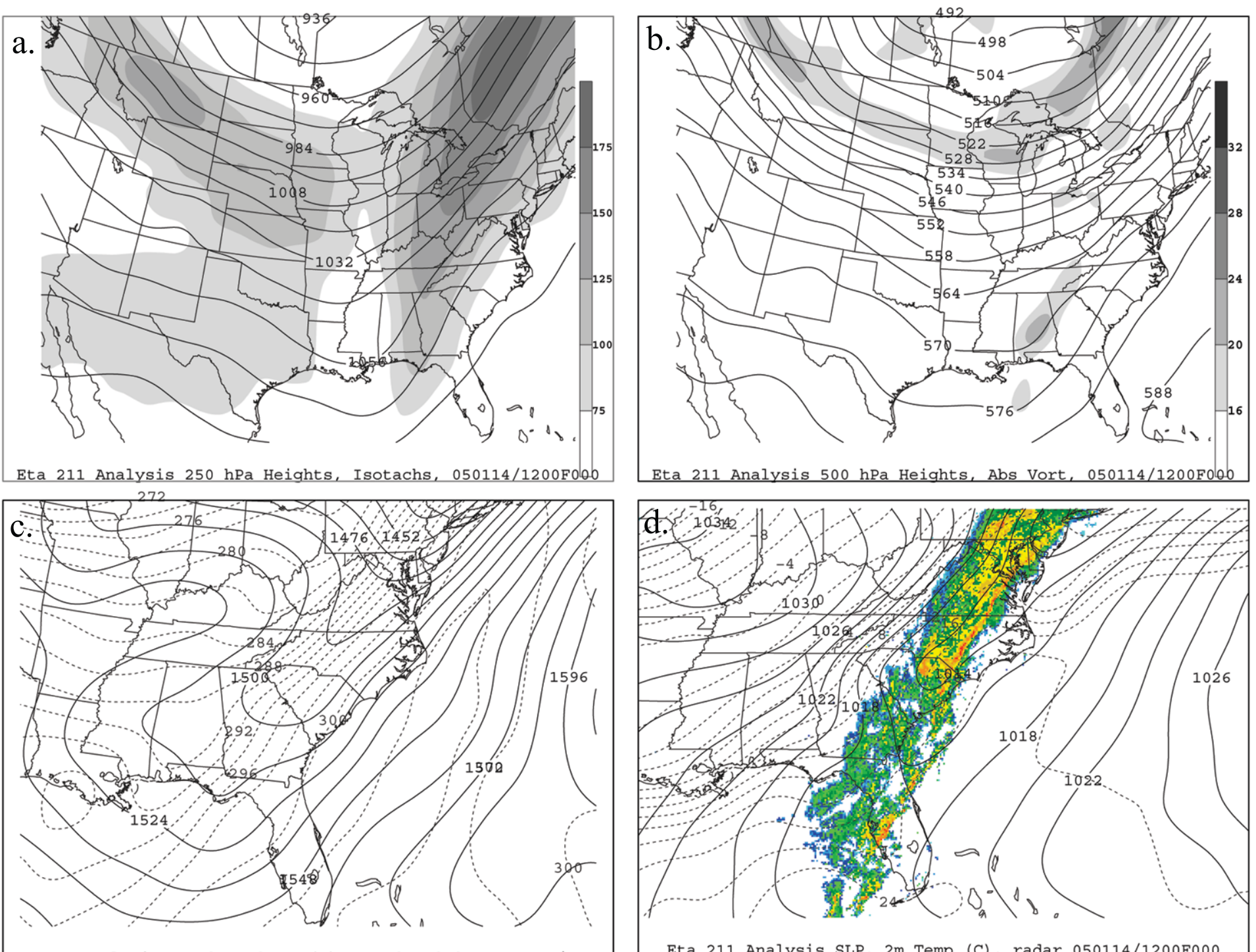

Figure 4.8. As in Fig. 4.5 except for 12 UTC 14 Jan. 

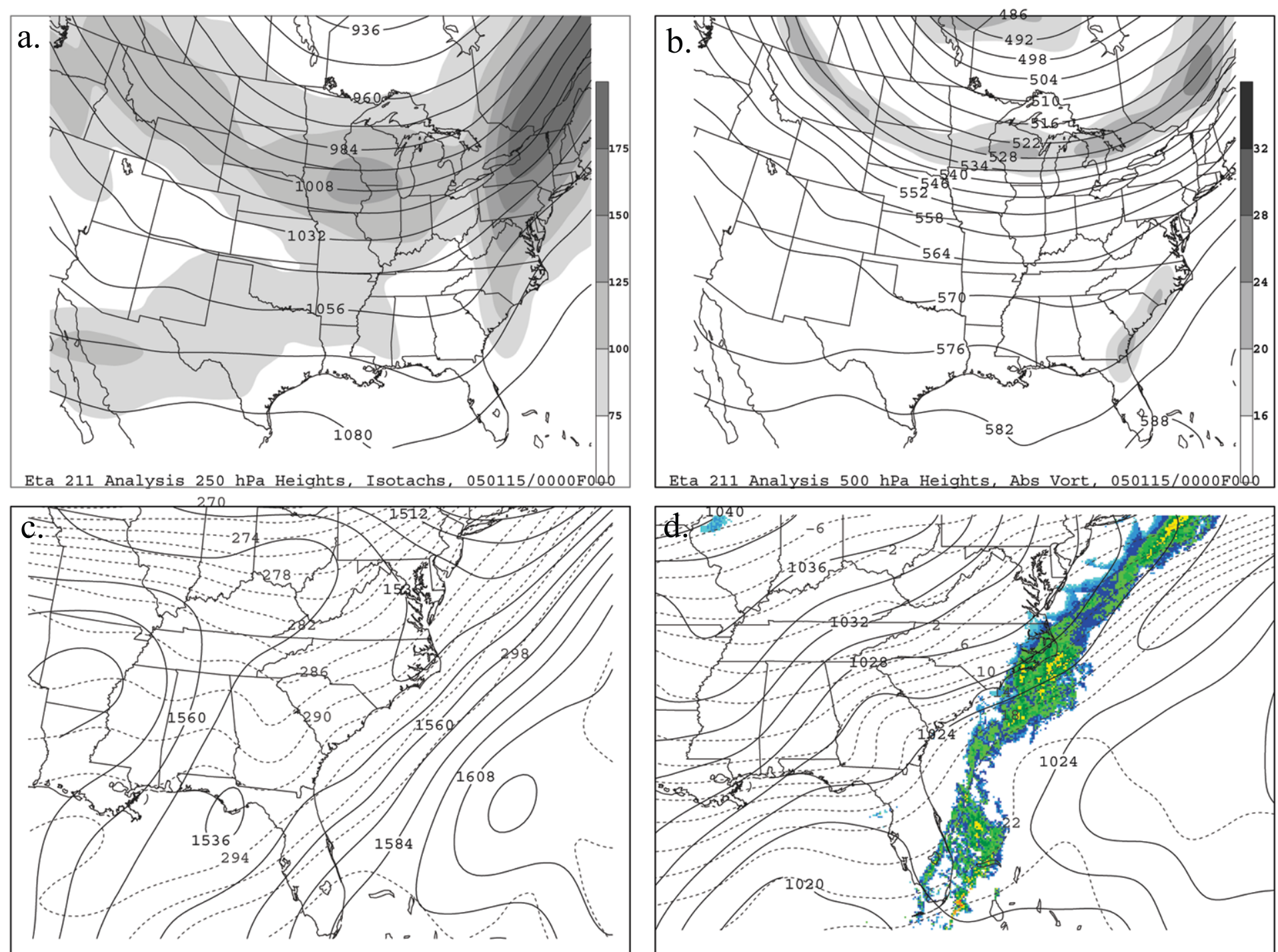

Eta 211 Analysis $850 \mathrm{hPa}$ theta (K), $850 \mathrm{hPa}$ hght, $050115 / 0000 \mathrm{H}$

Eta 211 Analysis SLP, 2m Temp (C), radar 050115/0000F000

Figure 4.9. As in Fig. 4.5 except for 00 UTC 15 Jan. 


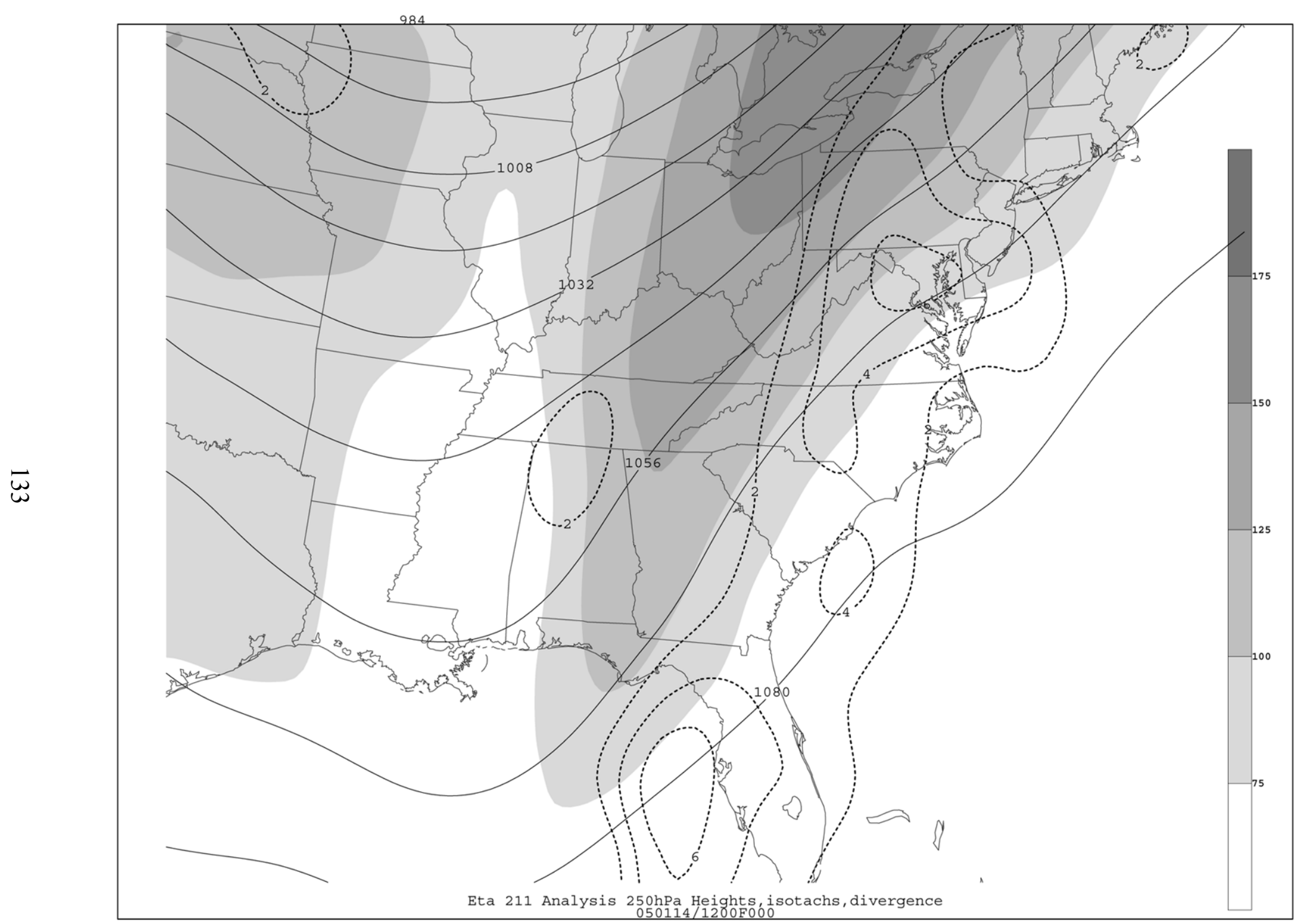

Figure 4.10. EDAS 250-hPa Geopotential height (solid contours, interval 12 dam), isotachs (kt, shaded as in legend at right of panel), and divergence (dashed contours, interval 2 x 10-5 s-1) valid 12 UTC 14 Jan. 
a.

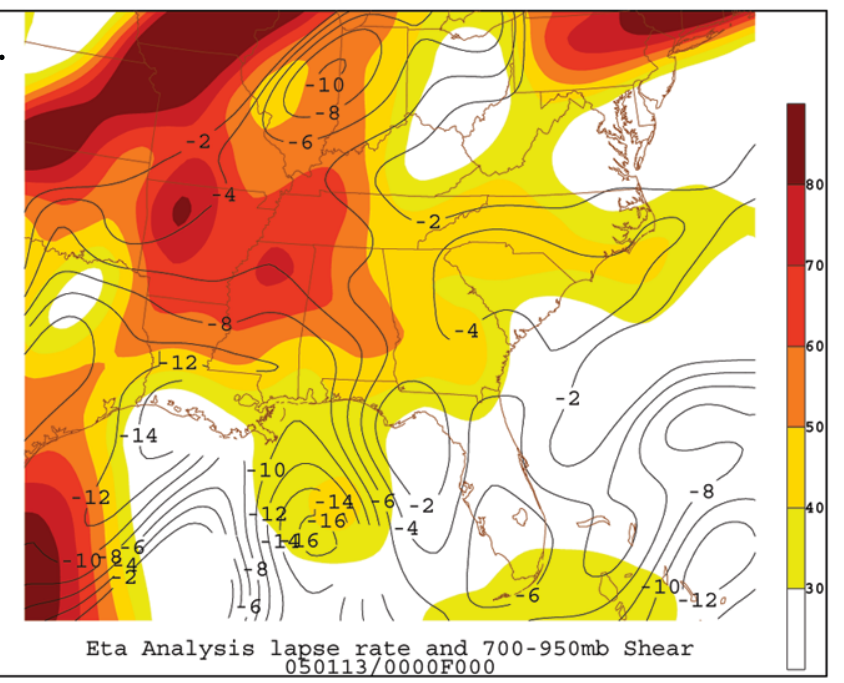

$\underset{\perp}{\breve{L}}$
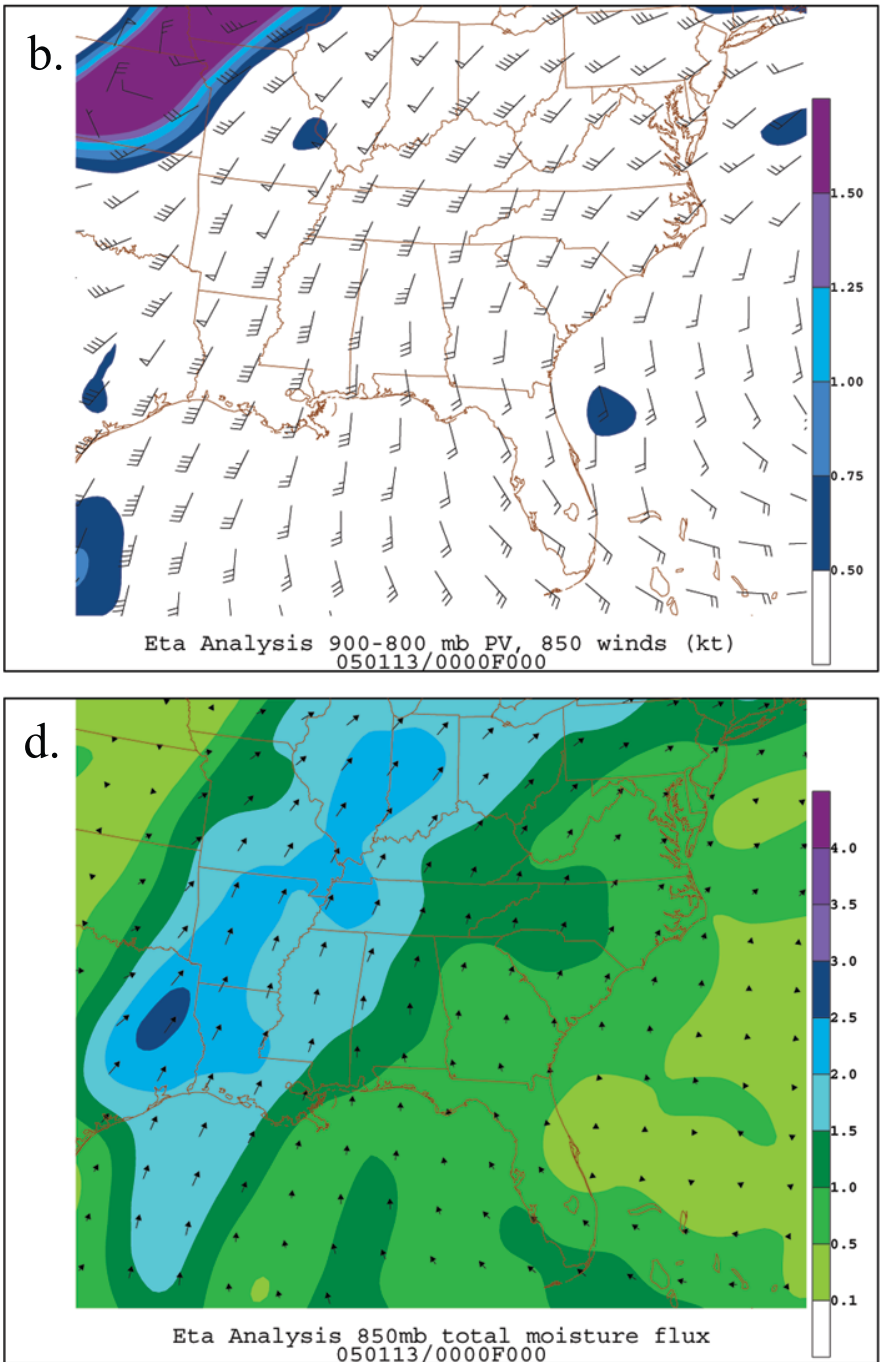

ta Analysis $850 \mathrm{mb}$ total moisture flux
Eta Analysis $850 \mathrm{mb}$ Heights, Isotachs, 050113/0000F000

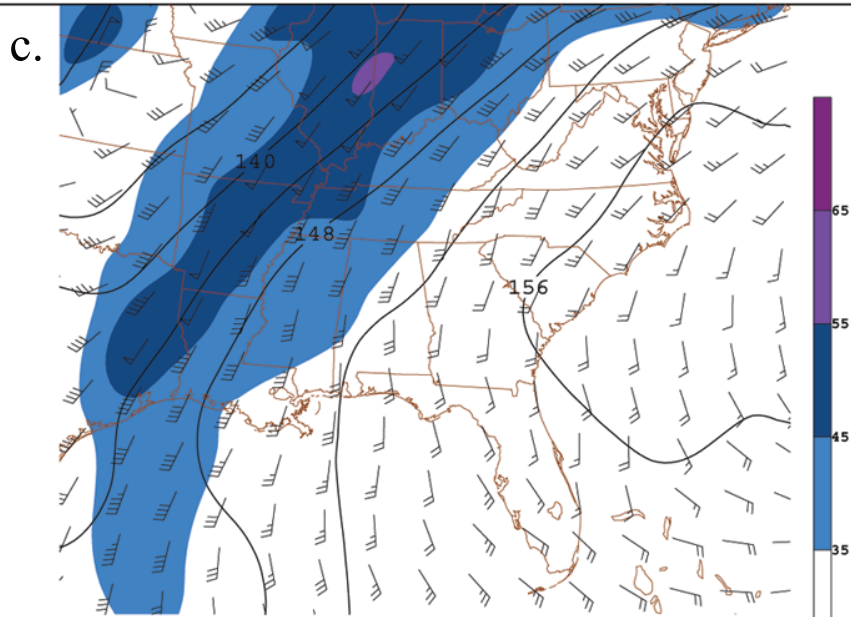

Figure 4.11. Eta Data Assimilation System (EDAS) analysis mesoscale summary for 00 UTC 13 Jan 2005: (a) 500-950-hPa equivalent potential temperature difference (solid contours, interval $2 \mathrm{~K}$, positive values omitted) and 700-950-hPa wind shear (10 kt/m, shaded as in legend at right of panel); (b) 900-800 layer-averaged PV (PVU, shaded as in legend at right of panel) and $850-\mathrm{hPa}$ winds (kt, barbs); (c) $850-\mathrm{hPa}$ Geopotential height (solid, interval 4 dam), isotachs (kt, shaded as in legend at right of panel), and 850-hPa winds (kt, barbs); (d) 850-hPa moisture flux (g kg-1 m s-1, shaded as in legend at right of panel) and moisture flux vectors (arrows). 

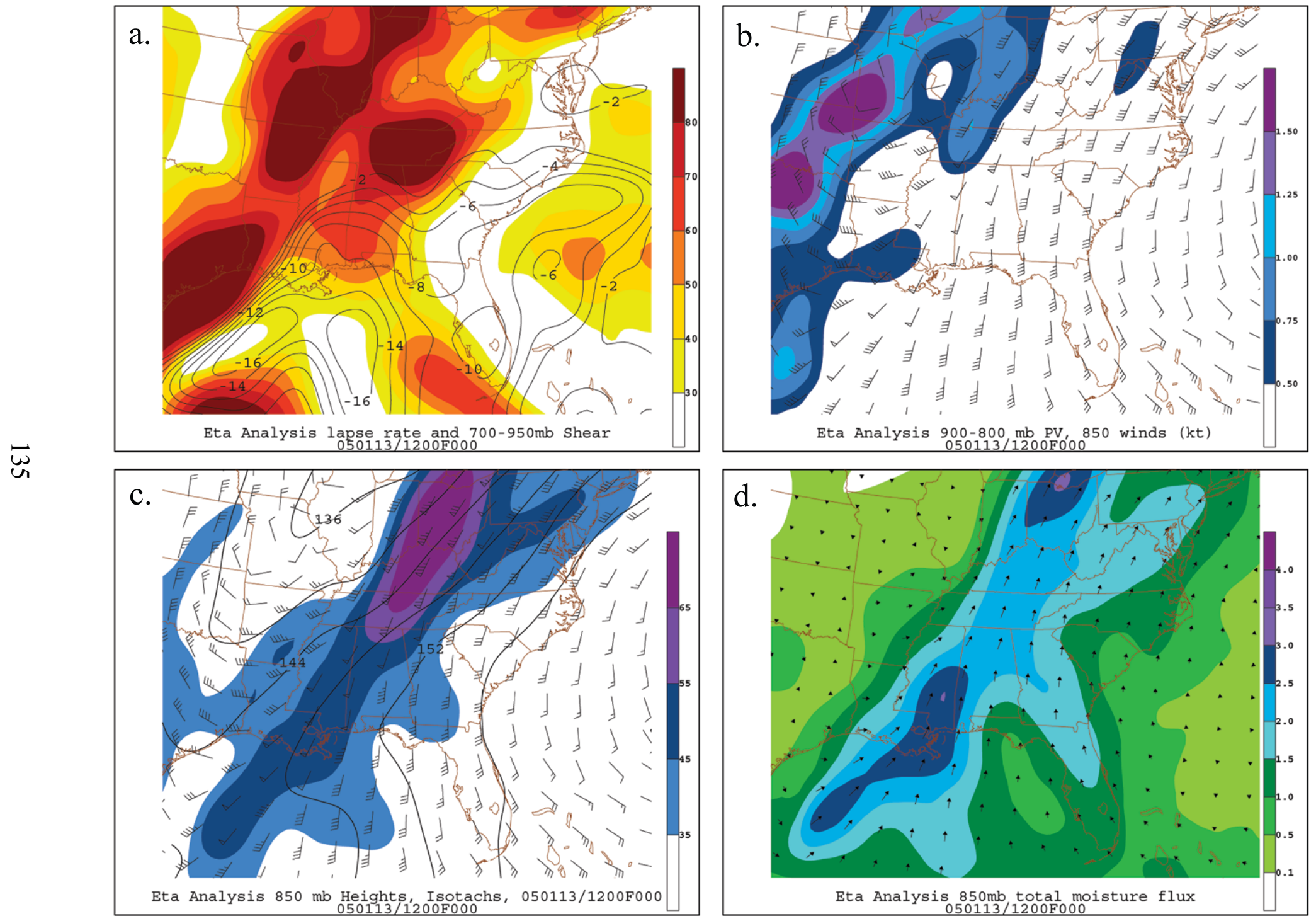

Figure 4.12. As in Fig. 4.11 except for 12 UTC 13 Jan. 

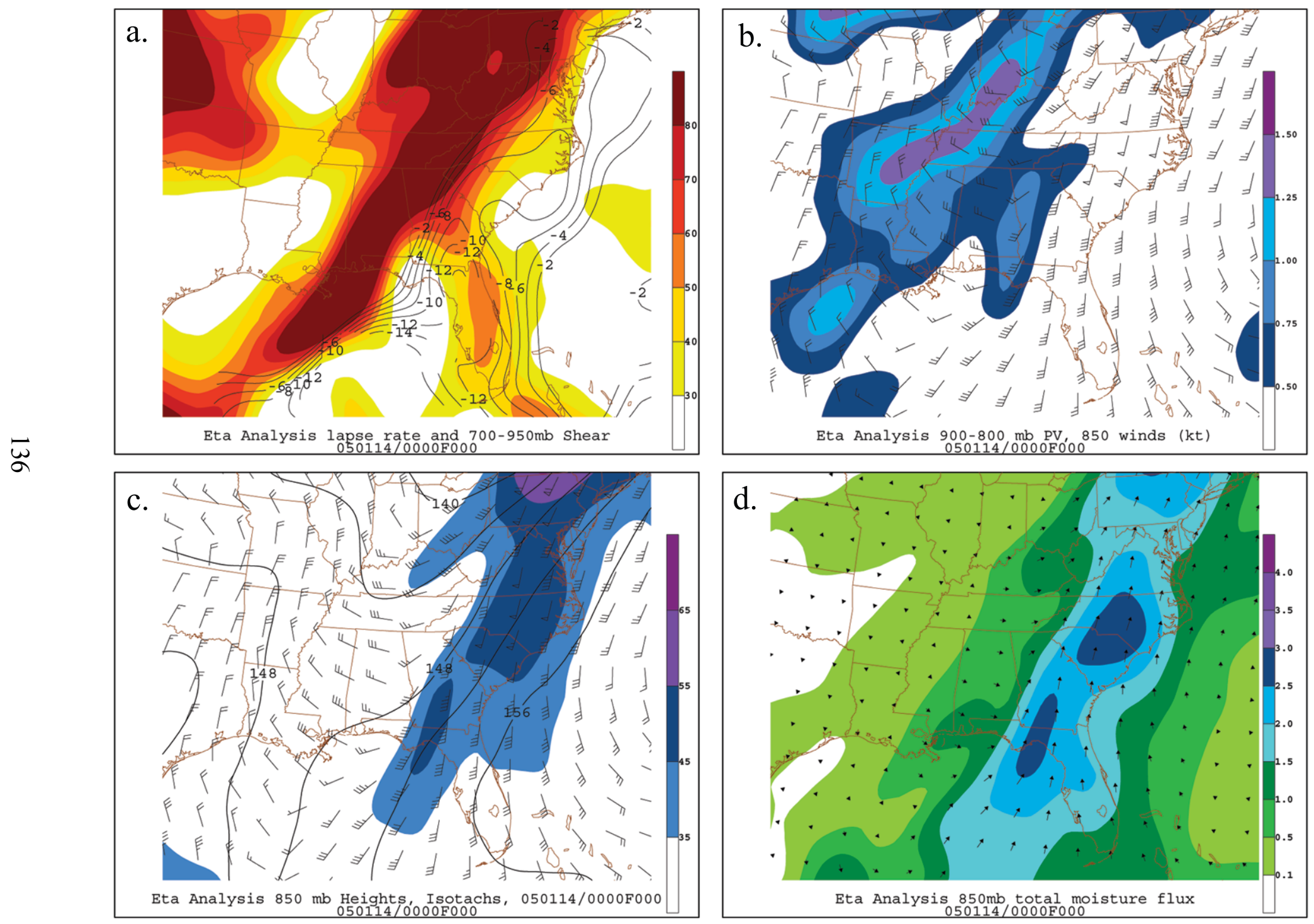

Figure 4.13. As in Fig. 4.11 except for 00 UTC 14 Jan. 


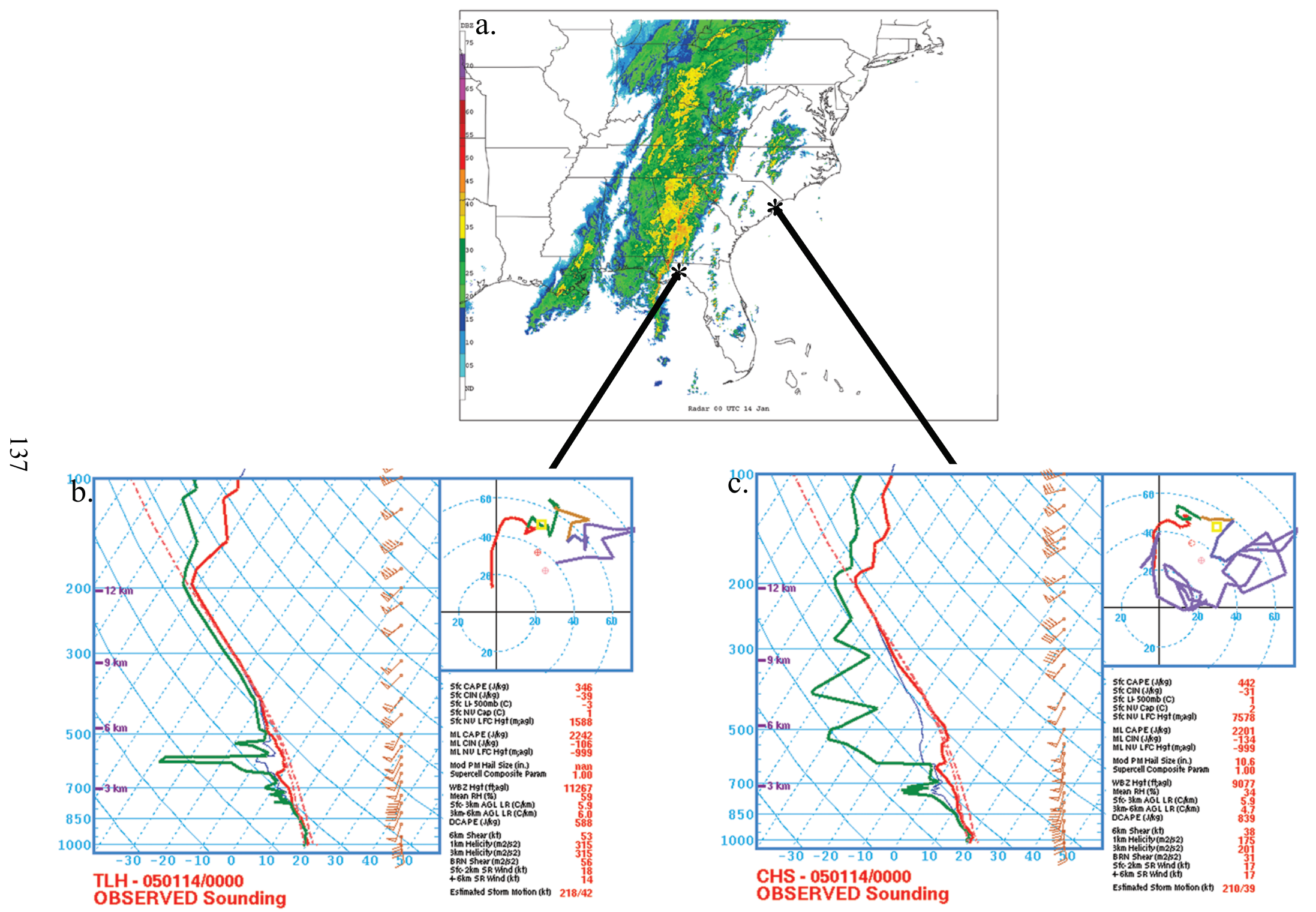

Figure 4.14. (a) NEXRAD radar reflectivity at 00 UTC 14 Jan; (b) observed TLH sounding at 00 UTC 14 Jan; (c) observed CHS sounding at 00 UTC 14 Jan. 

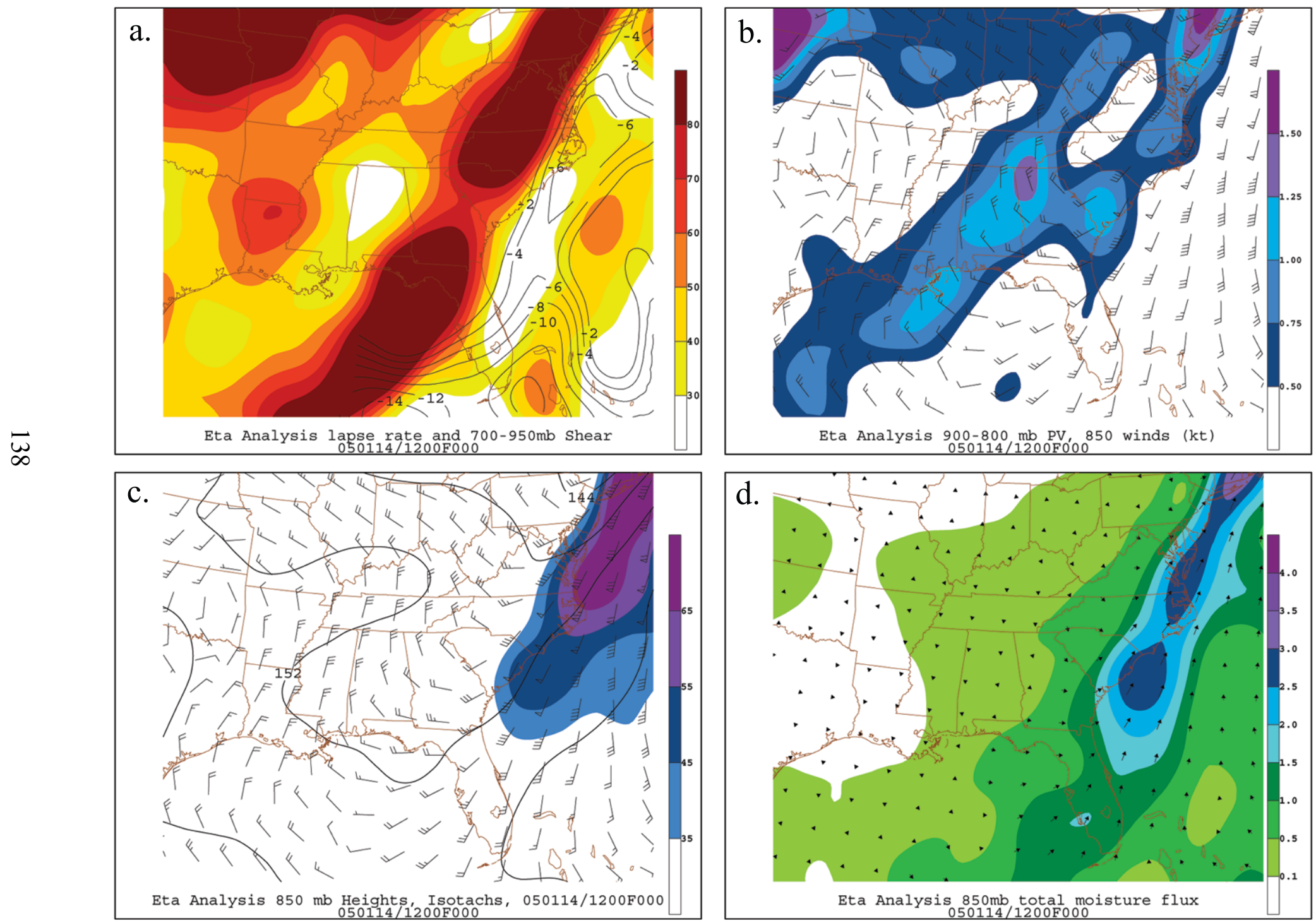

Figure 4.15. As in Fig. 4.11 except for 12 UTC 14 Jan. 

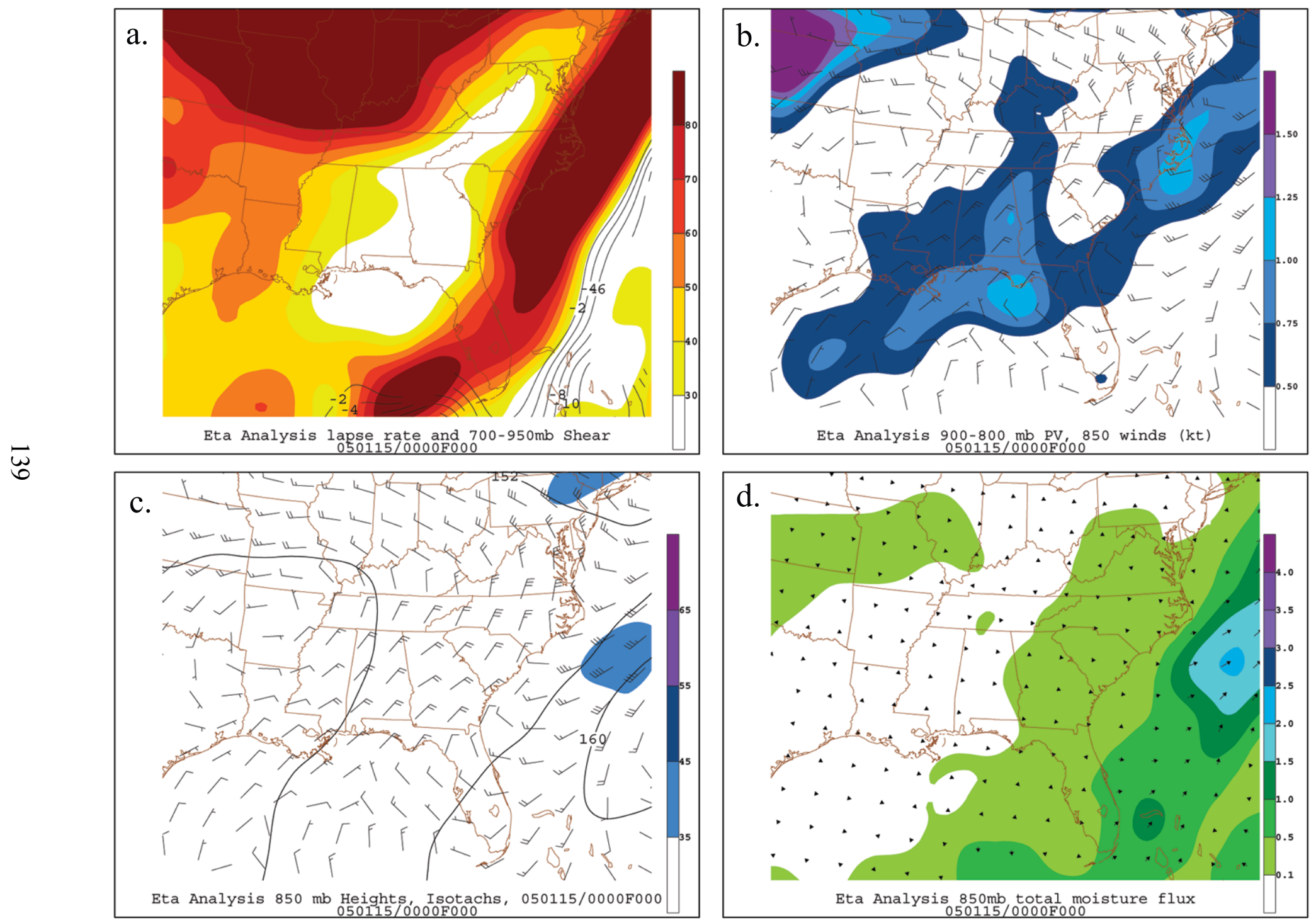

Figure 4.16. As in Fig. 4.11 except for 00 UTC 15 Jan. 

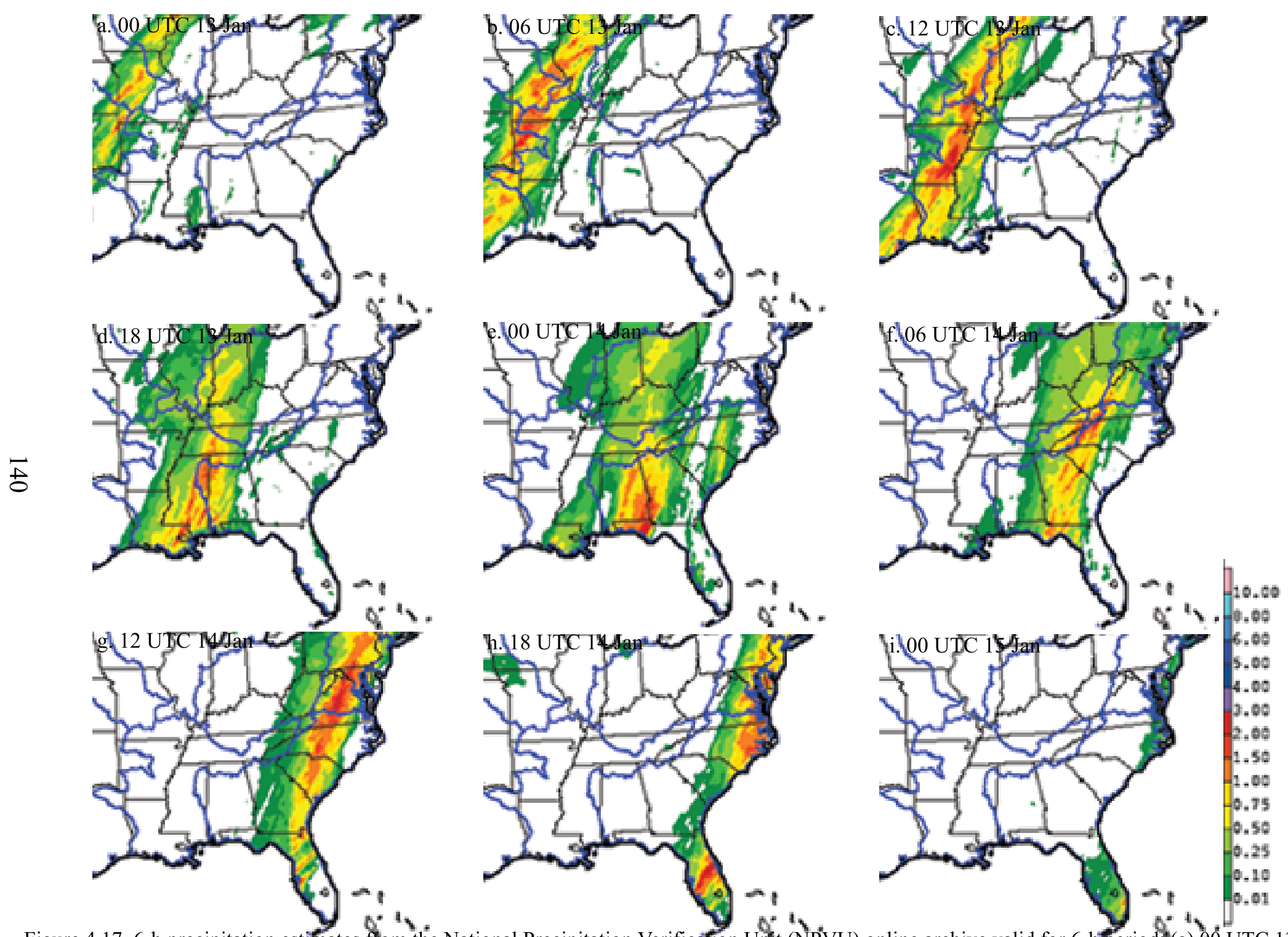

Figure 4.17. 6-h precipitation estimates ffom the National Precipitation Verification Unit (NPEV) online archive valid for 6-h period:(a) 00 UTC 13 Jan; (b) 06 UTC 13 Jan; (c) 12 UTC 13 Jan; (d) 18 UTC 13 Jan; (e) 00 UTC 14 Jan; (f) 06 UTC 14 Jan; (g) 12 UTC 14 Jan; (h) 18 UTC 14 Jan; (i) 00 UTC 15 Jan. 
Figure 4.18. 6-h QPFs from operational Eta model initialized 00 UTC 13 Jan, valid for 6-h period ending: (a) (omitted; no QPF at F000); (b) 06 UTC 13 Jan; (c) 12 UTC 13 Jan; (d) 18 UTC 13 Jan; (e) 00 UTC 14 Jan; (f) 06 UTC 14 Jan; (g) 12 UTC 14 Jan; (h) 18 UTC 14 Jan; (i) 00 UTC 15 Jan.
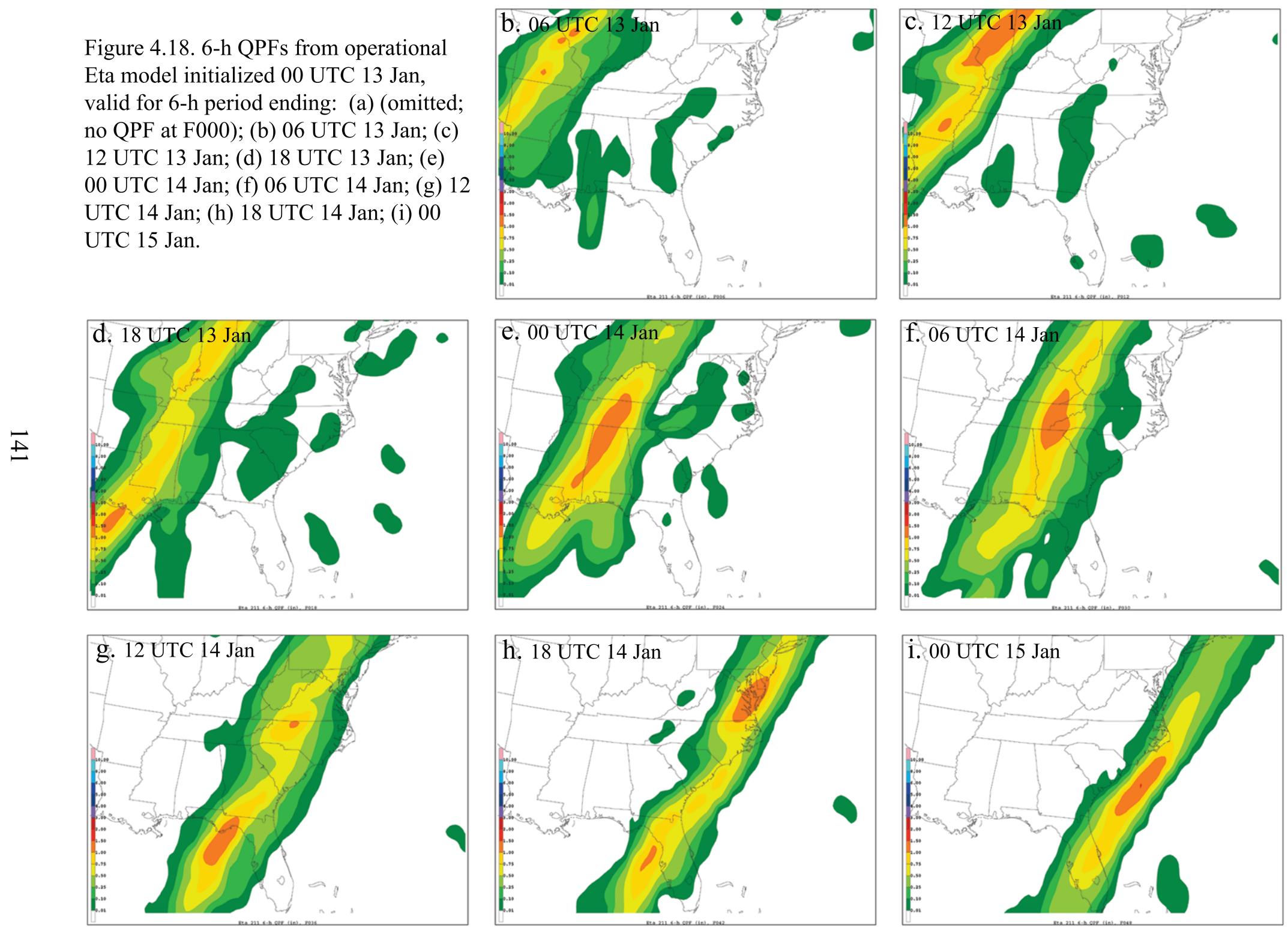

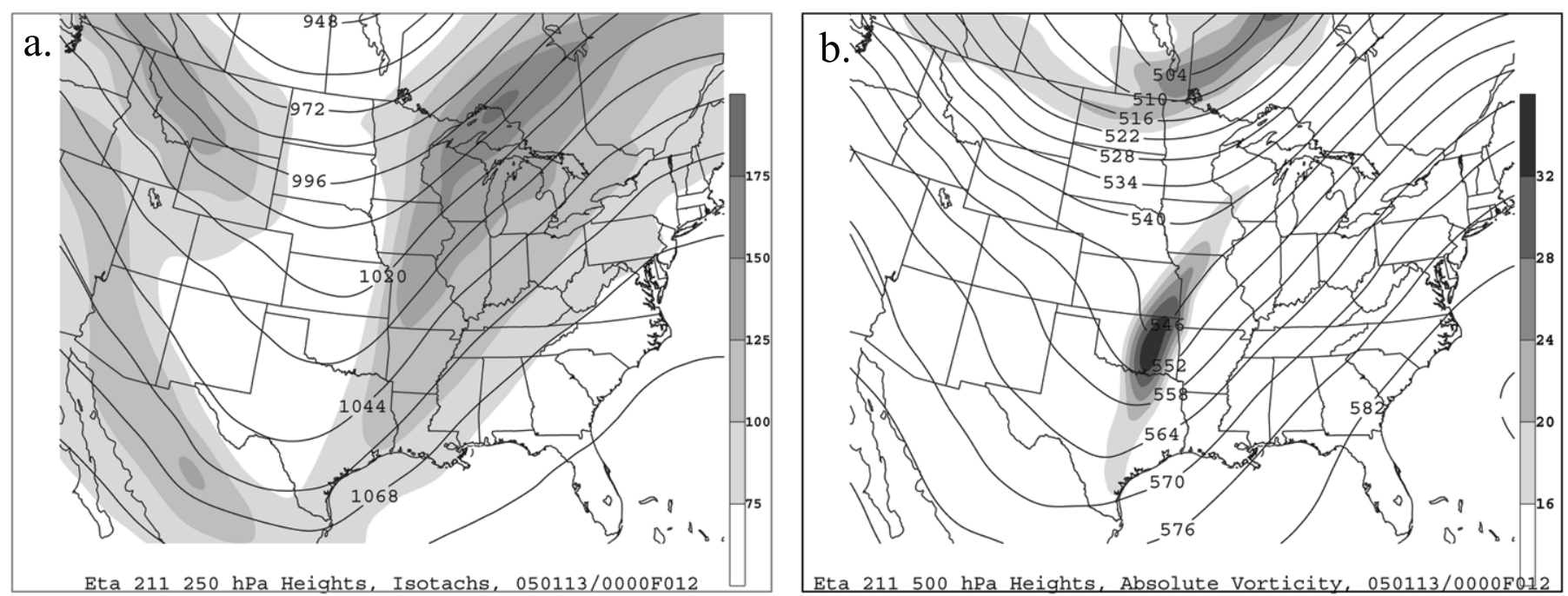

点
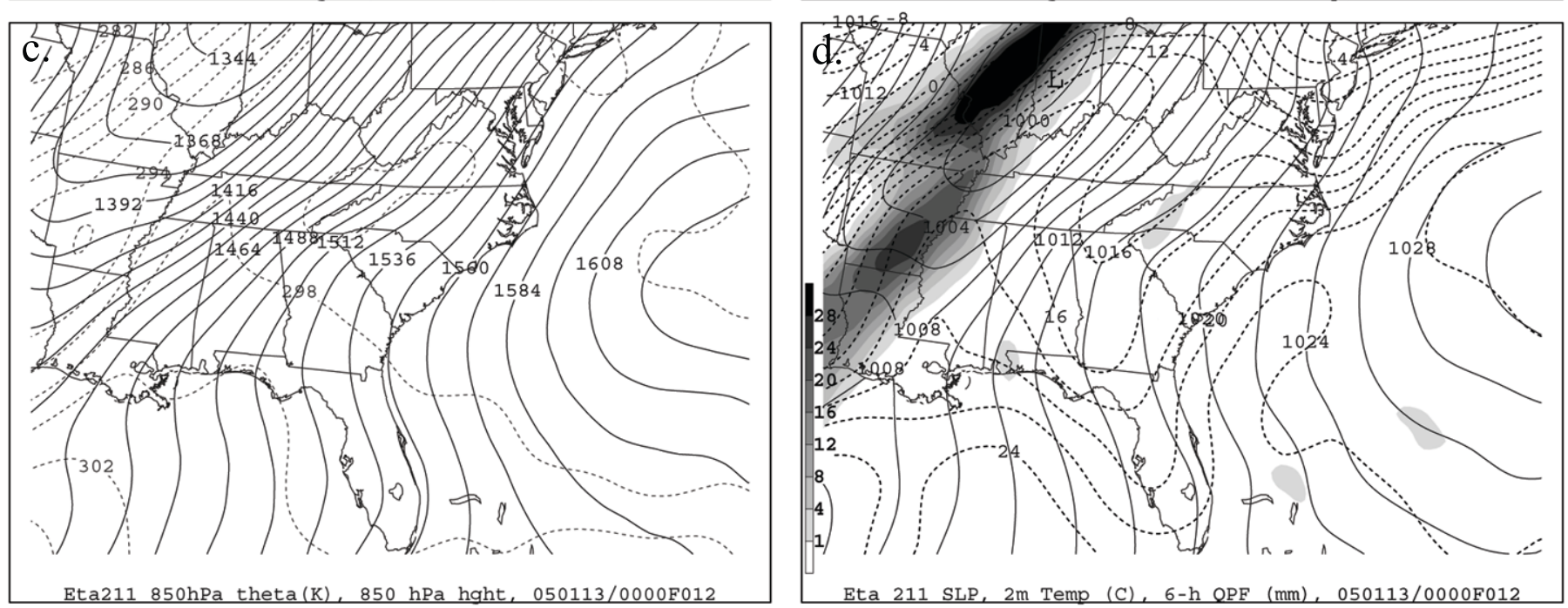

Figure 4.19. Operational Eta 12-h forecast valid 12 UTC 13 Jan 2005: (a) 250-hPa Geopotential height (solid contours, interval $12 \mathrm{dam}$ ) and isotachs (kt, shaded as in legend at right of panel); (b) 500-hPa Geopotential height (solid, interval $6 \mathrm{dam}$ ) and vorticity ( $\mathrm{x} 10^{\wedge}-5 \mathrm{~s}^{\wedge}-1$, shaded as in legend at right of panel); (c) 850-hPa potential temperature (dashed contours, interval $2 \mathrm{~K}$ ), 850-hPa Geopotential height (solid, interval 6 dam), and 850-hPa mixing ratio (shaded, interval $2 \mathrm{~g} / \mathrm{kg}$, values below $6 \mathrm{~g} / \mathrm{kg}$ omitted); (d) sea level pressure (solid contours, interval $2 \mathrm{hPa}$ ), 2-m temperature (dashed contours, interval 2C), and 6-h accumulated precipitation (mm, shaded as in legend at left of panel) ending $12 \mathrm{UTC} 13$ Jan. 

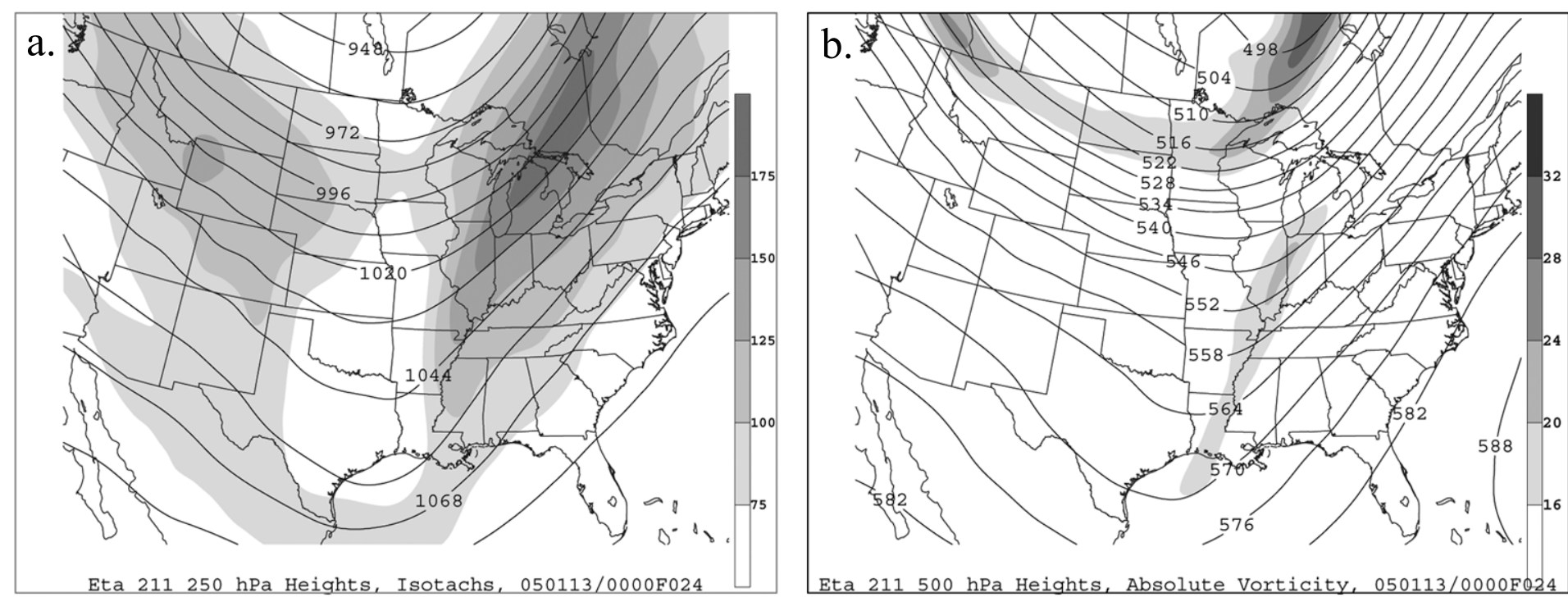

E
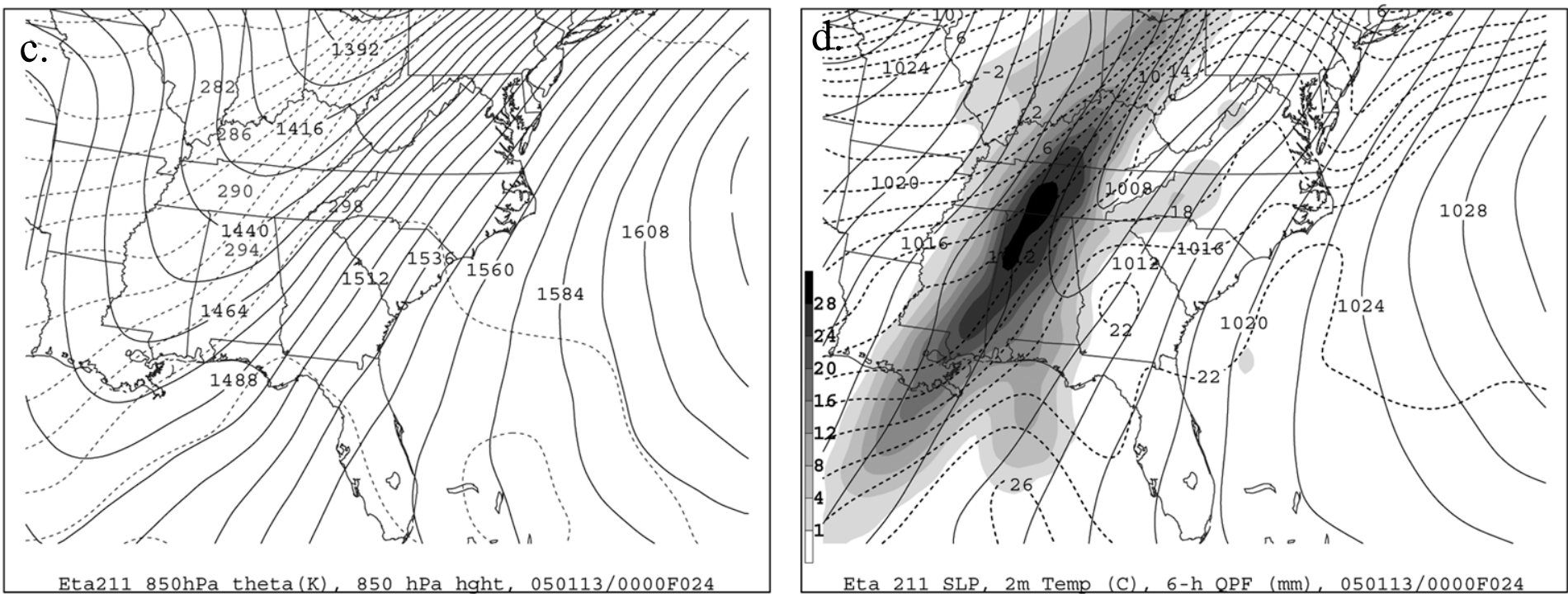

Figure 4.20. As in Fig. 4.19 except for 00 UTC 14 Jan. 

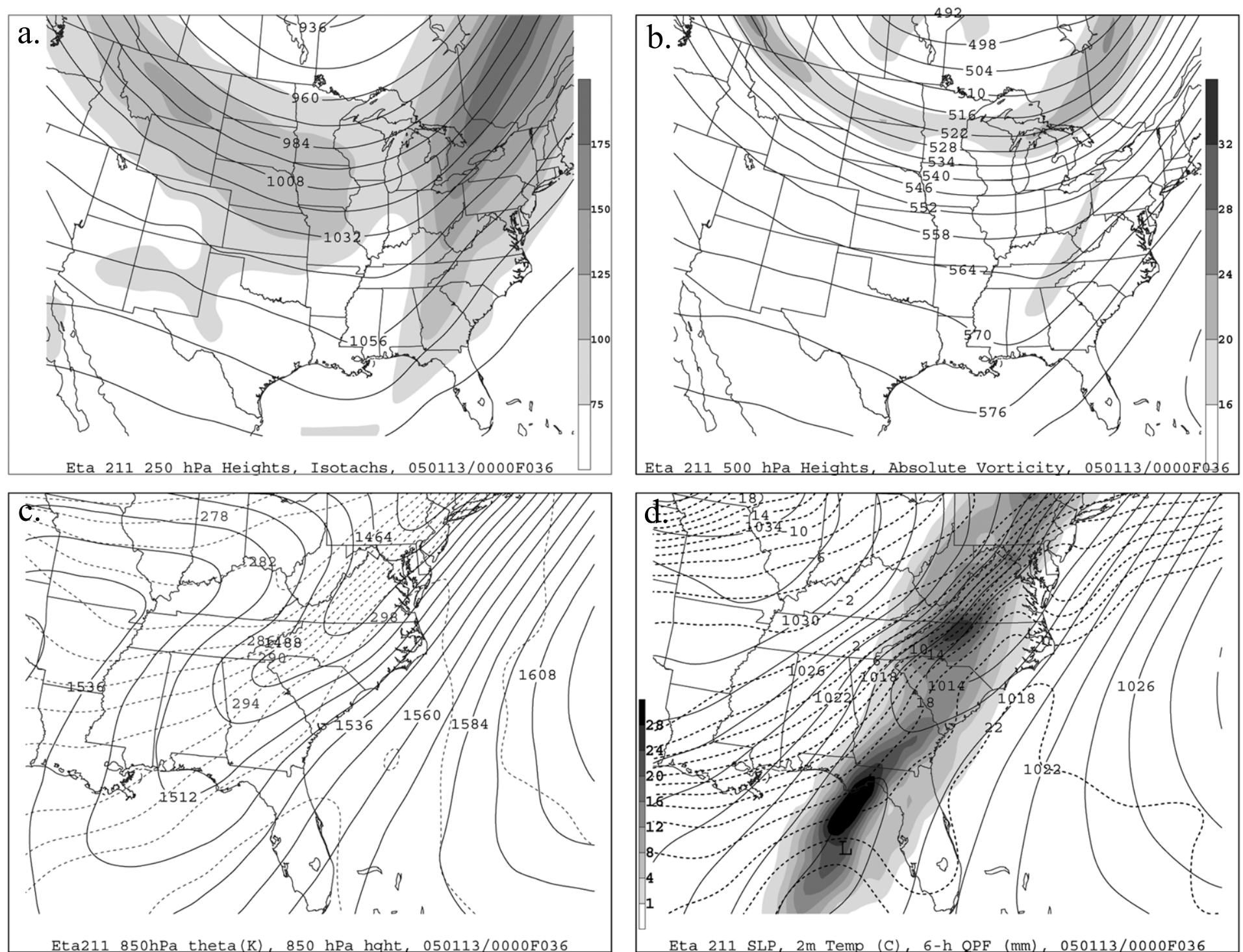

Figure 4.21. As in Fig. 4.19 except for 12 UTC 14 Jan. 

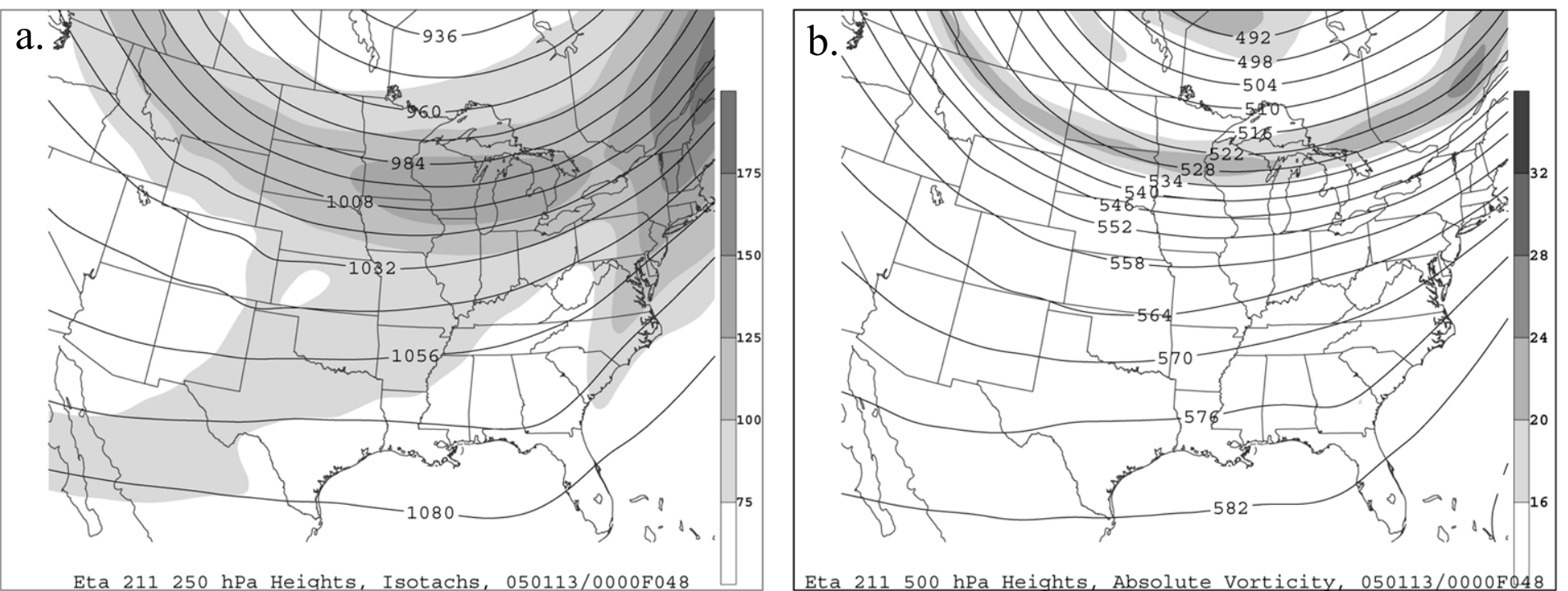

点
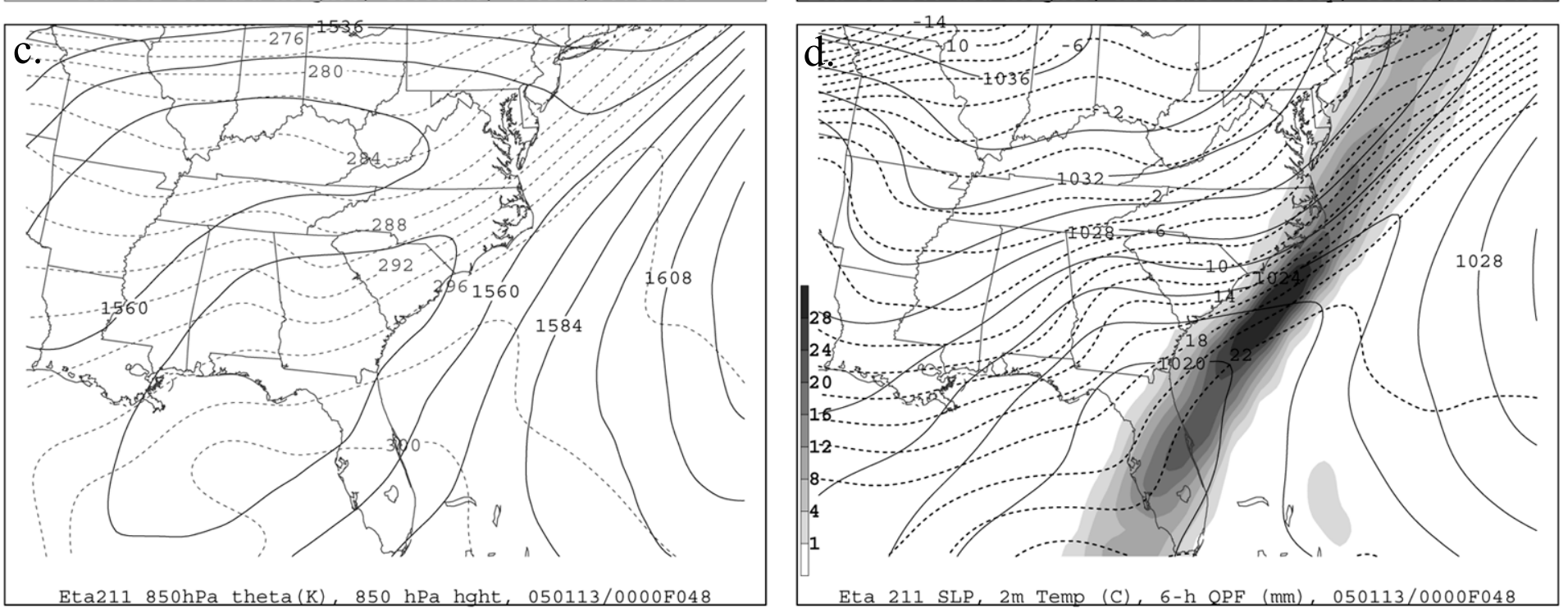

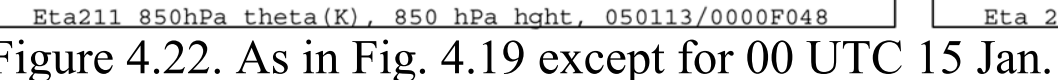



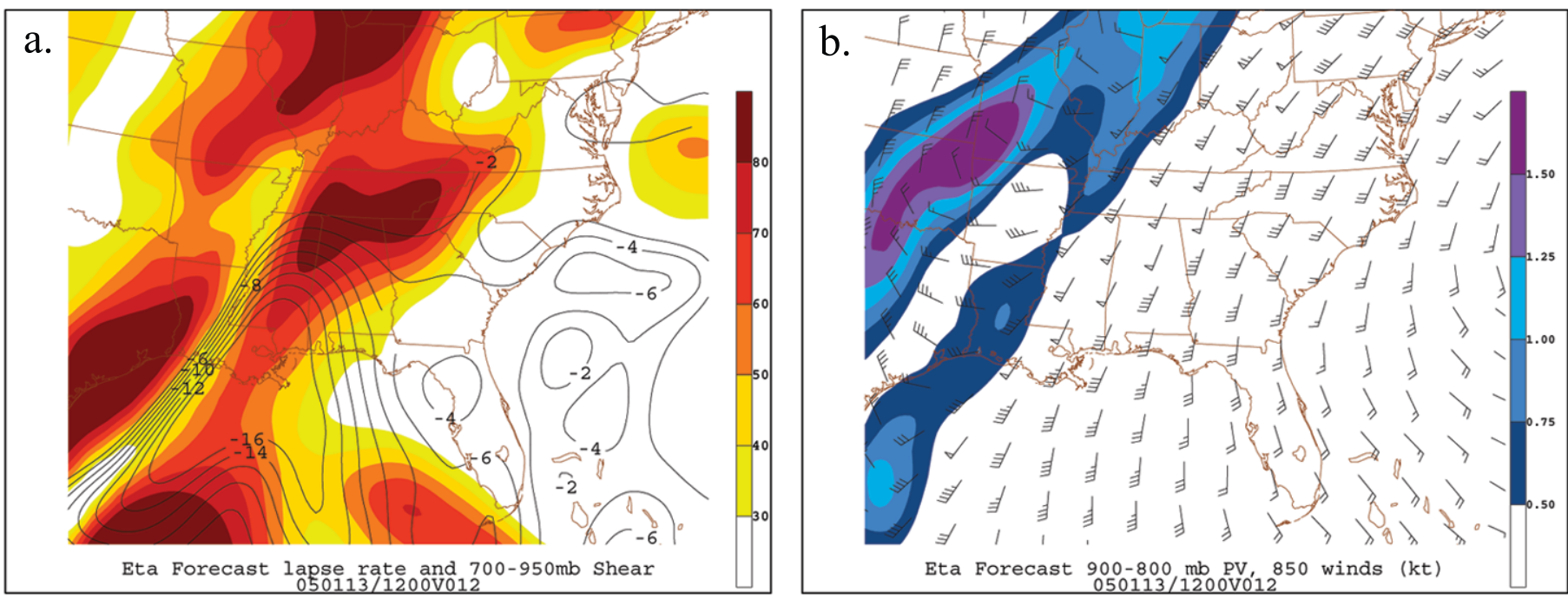

点
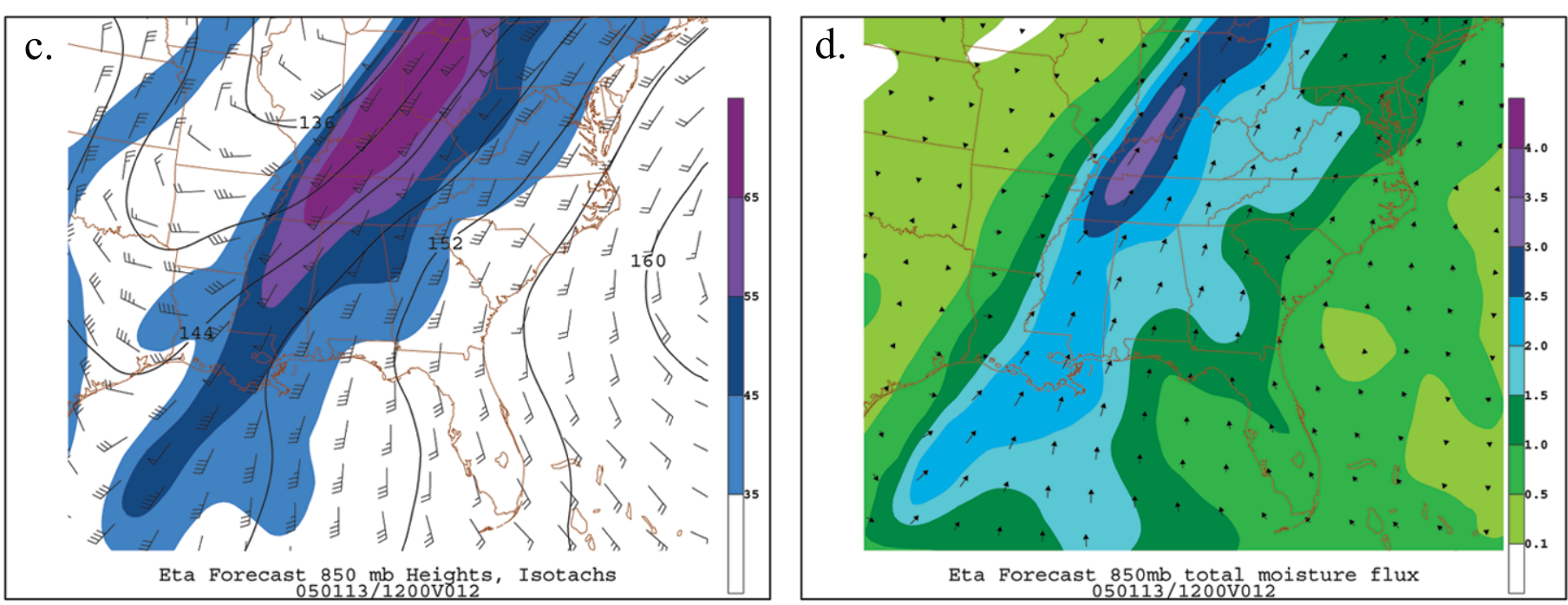

Figure 4.23. Operational Eta 12-h forecast valid 12 UTC 13 Jan 2005: (a) 500-950-hPa equivalent potential temperature difference (solid contours, interval 2 K, positive values omitted) and 700-950-hPa wind shear (10 kt/m, shaded as in legend at right of panel); (b) 900-800 layer-averaged PV (PVU, shaded as in legend at right of panel) and 850-hPa winds (kt, barbs); (c) 850-hPa Geopotential height (solid contours, interval 4 dam), isotachs (kt, shaded as in legend at right of panel), and 850-hPa winds (kt, barbs); (d) 850-hPa moisture flux (g kg-1 m s-1, shaded as in legend at right of panel) and moisture flux vectors (arrows). 

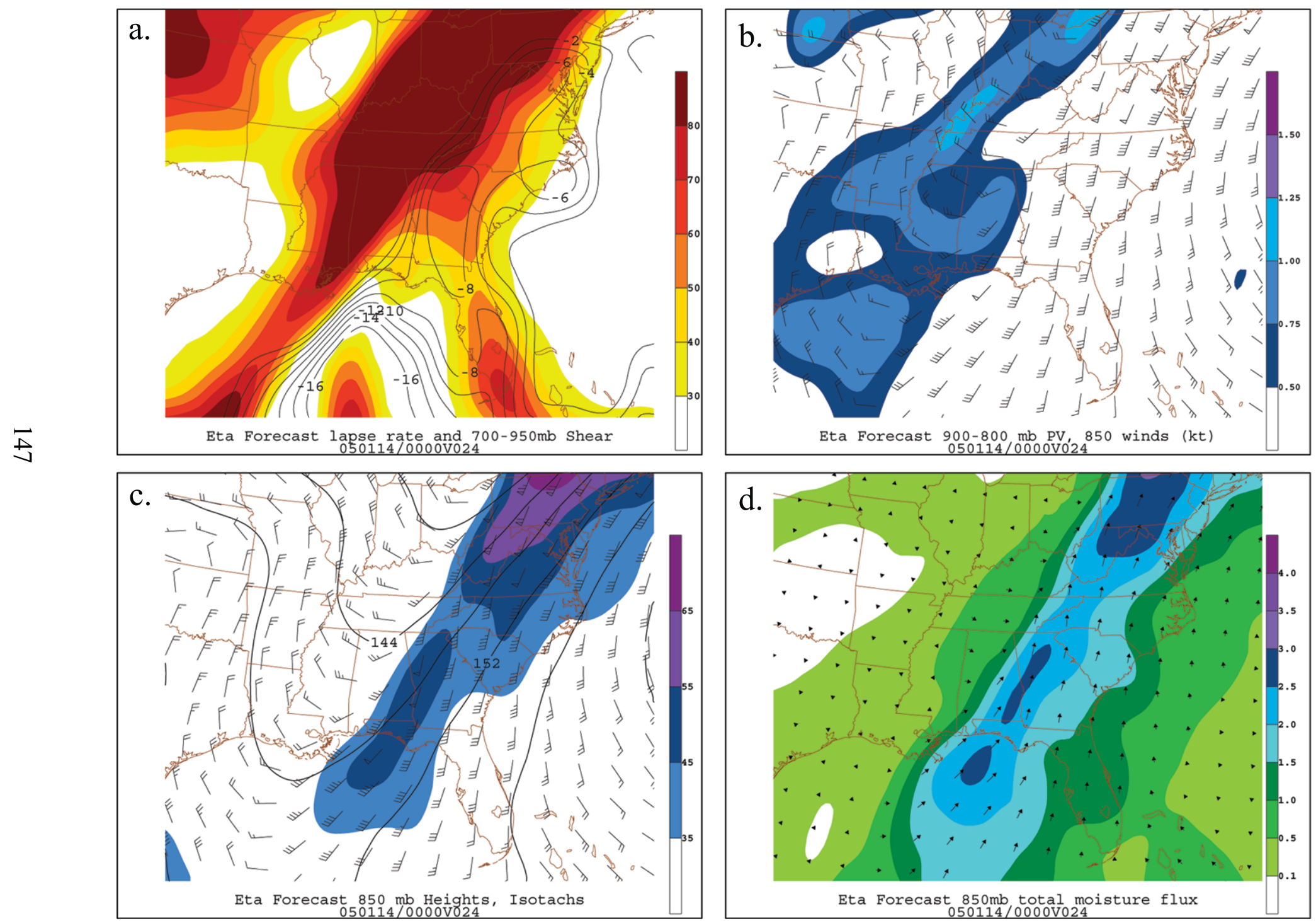

Figure 4.24. As in Fig. 4.23 except for 00 UTC 14 Jan. 

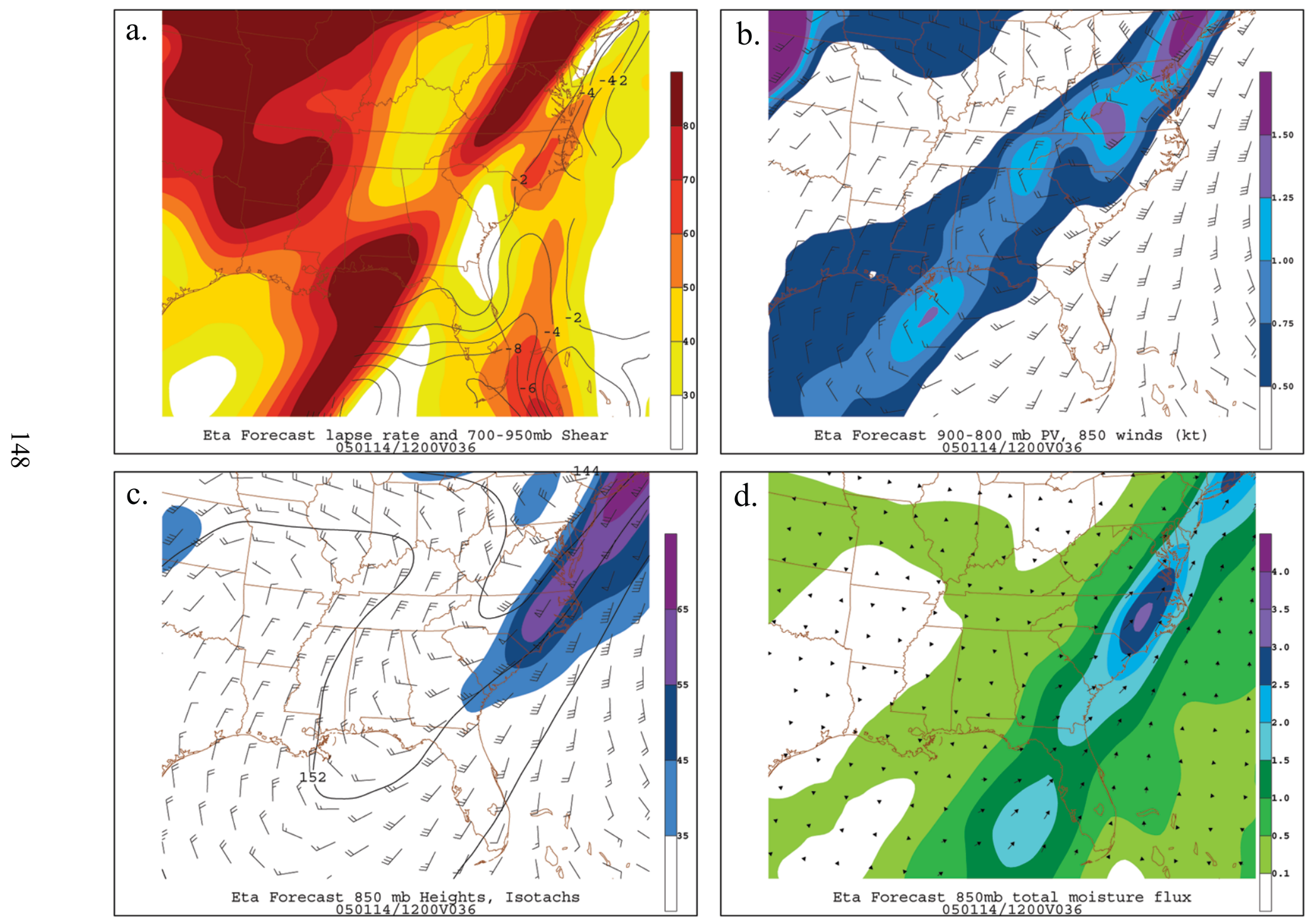

Figure 4.25. As in Fig. 4.23 except for 12 UTC 14 Jan. 

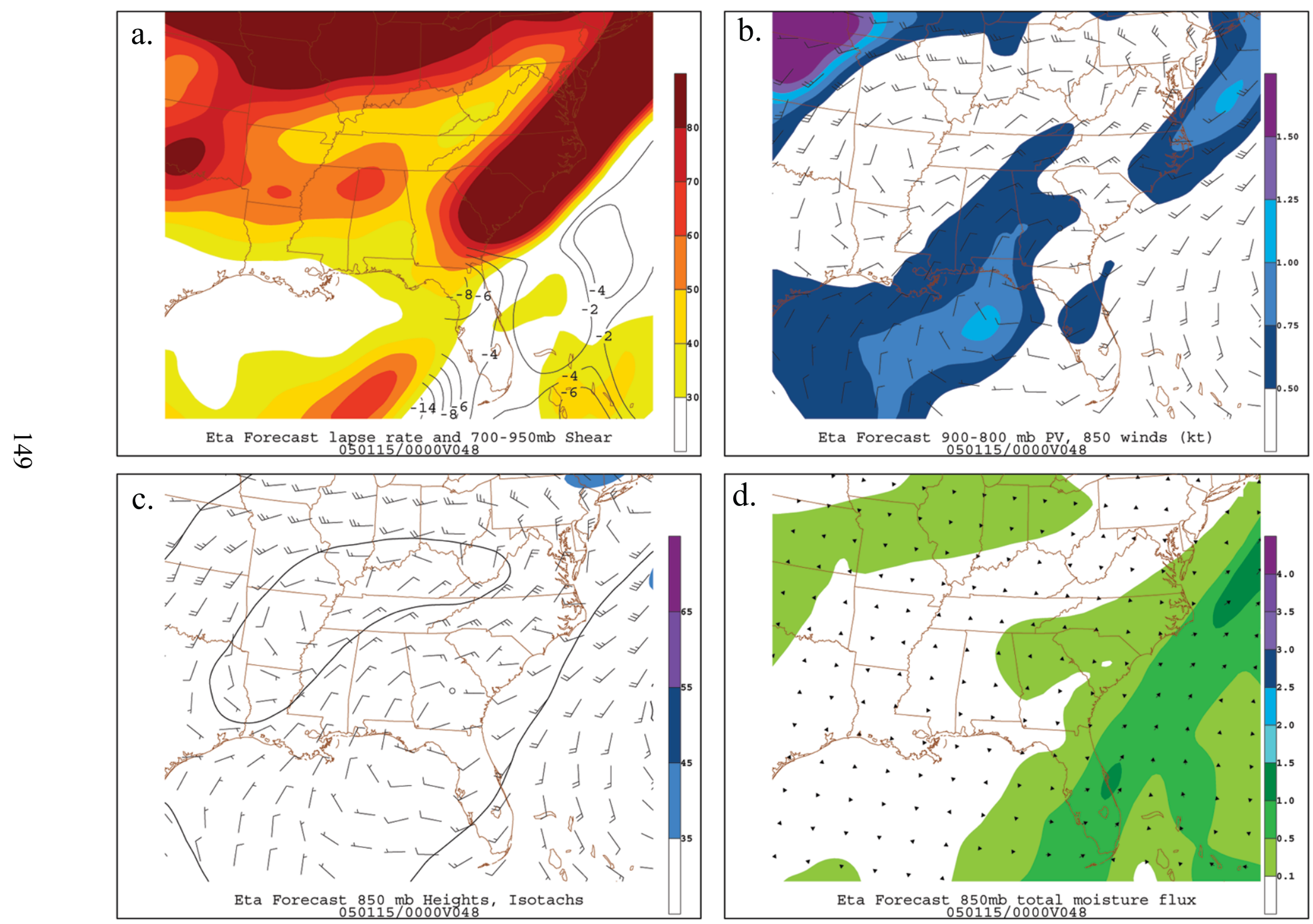

Figure 4.26. As in Fig. 4.23 except for 00 UTC 15 Jan. 


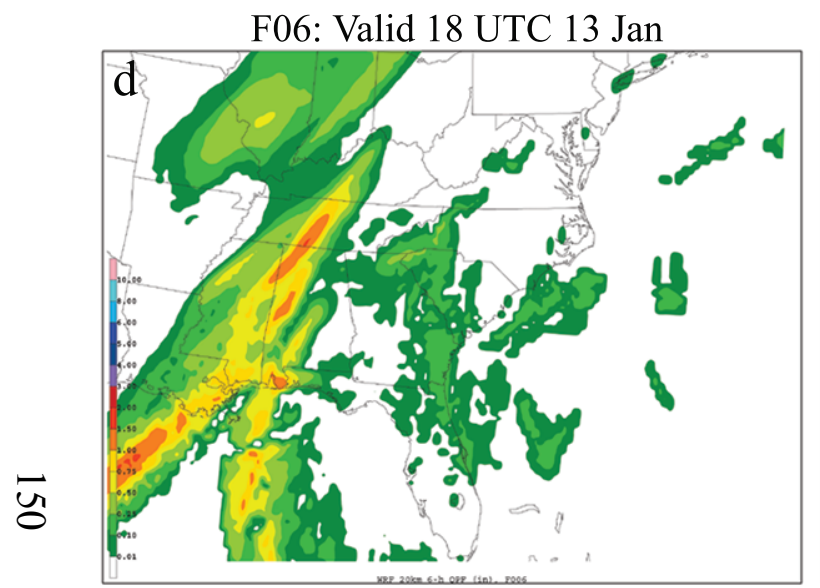

F24: Valid 12 UTC 14 Jan
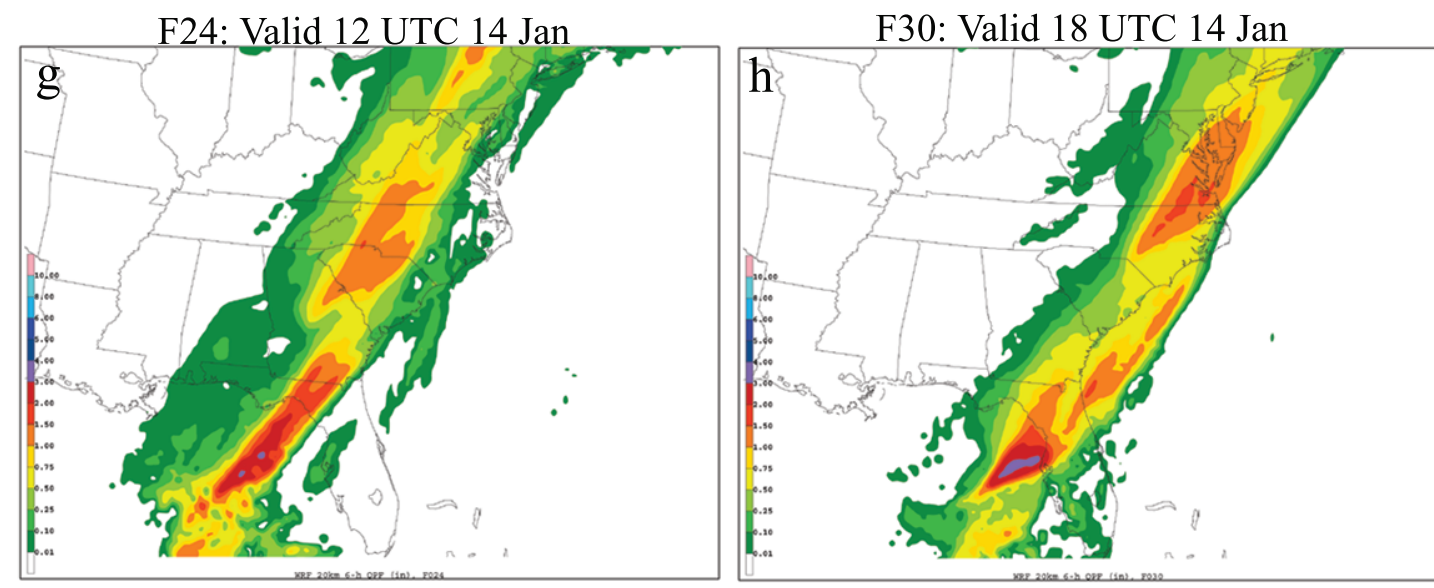

F12: Valid 00 UTC 14 Jan

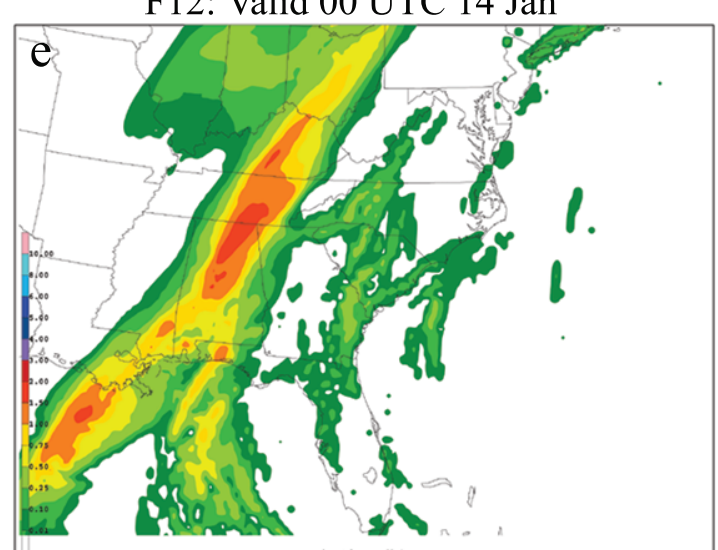

.

F18: Valid 06 UTC 14 Jan

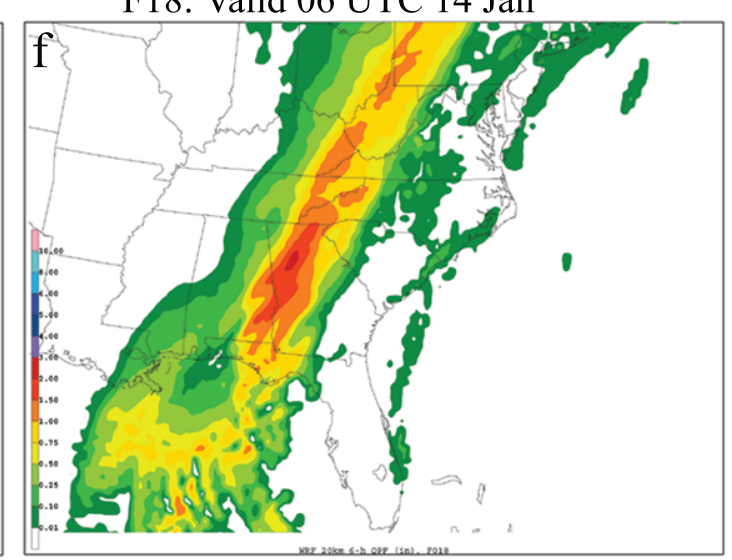

F36: Valid 00 UTC 15 Jan

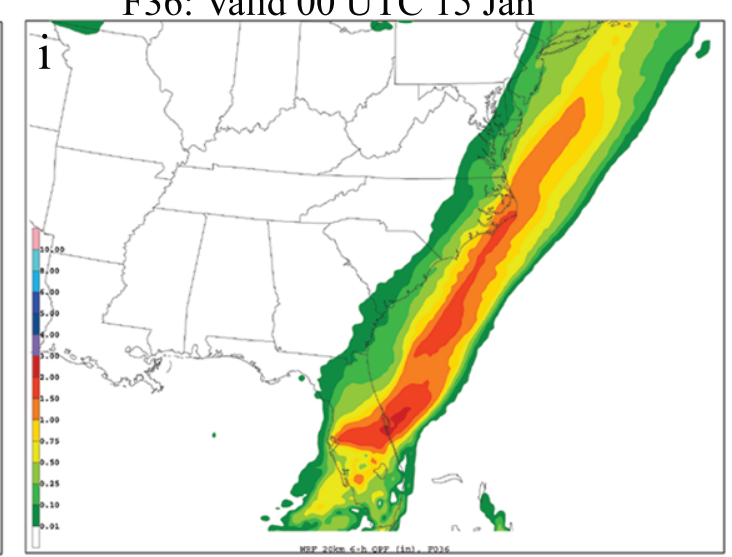

Figure 4.27. 6-h QPFs from WRF model forecast. Valid for 6-h period ending: [(a)-(c) omitted to better match previous precipitation plots] (d) 18 UTC 13 Jan; (e) 00 UTC 14 Jan; (f) 06 UTC 14 Jan; (g) 12 UTC 14 Jan; (h) 18 UTC 14 Jan; (i) 00 UTC 15 Jan. 


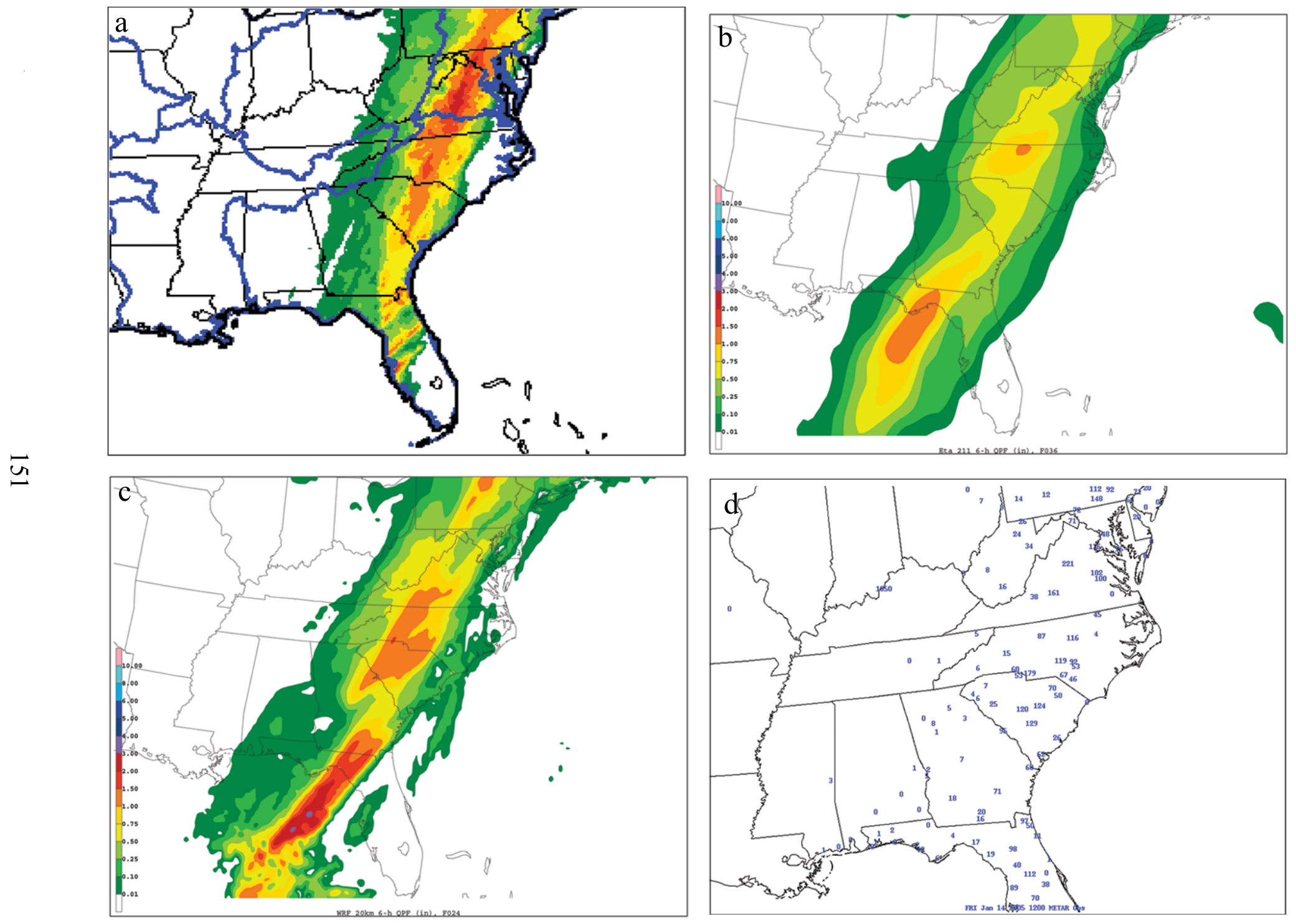

Figure 4.28 (a) NPVU 6-h precipitation analysis for 6-h period ending 12 UTC 14 Jan (inches, shaded as in legend at right of panels (b) and (c)); (b) as in (a) except for operational Eta model QPF; (c) as in (a) except for WRF QPF; (d) 6-h METAR observed precipitation totals (numbers, hundredths of inches) 

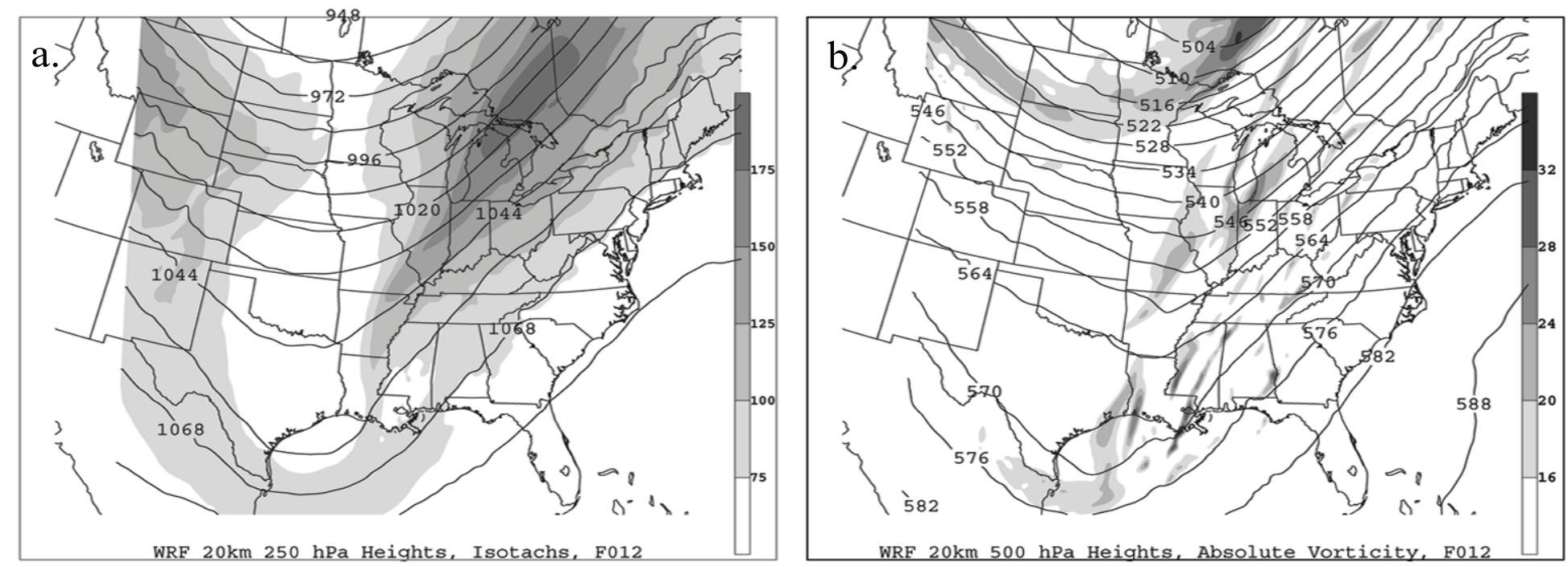

岕
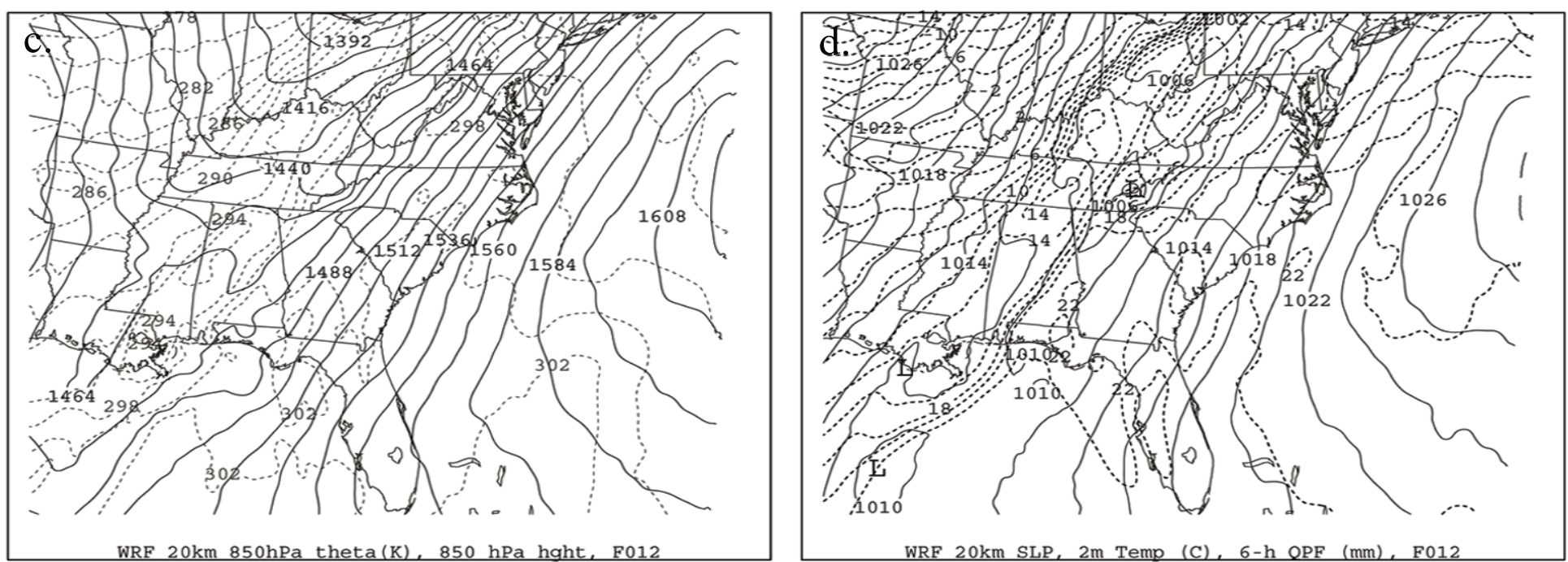

Figure 4.29. WRF 12-h forecast valid 00 UTC 14 Jan 2005: (a) 250-hPa Geopotential height (solid contours, interval 12 dam) and isotachs (kt, shaded as in legend at right of panel); (b) 500$\mathrm{hPa}$ Geopotential height (solid, interval $6 \mathrm{dam})$ and vorticity ( $\times 10^{\wedge}-5 \mathrm{~s}^{\wedge}-1$, shaded as in legend at right of panel); (c) 850-hPa potential temperature (dashed contours, interval $2 \mathrm{~K}$ ), 850-hPa Geopotential height (solid, interval $6 \mathrm{dam}$ ), and $850-\mathrm{hPa}$ mixing ratio (shaded, interval $2 \mathrm{~g} / \mathrm{kg}$, values below $6 \mathrm{~g} / \mathrm{kg}$ omitted); (d) sea level pressure (solid contours, interval $2 \mathrm{hPa}$ ), 2 - $\mathrm{m}$ temperature (dashed contours, interval 2C), and 6-h accumulated precipitation (mm, shaded as in legend at left of panel) ending 12 UTC 13 Jan. 

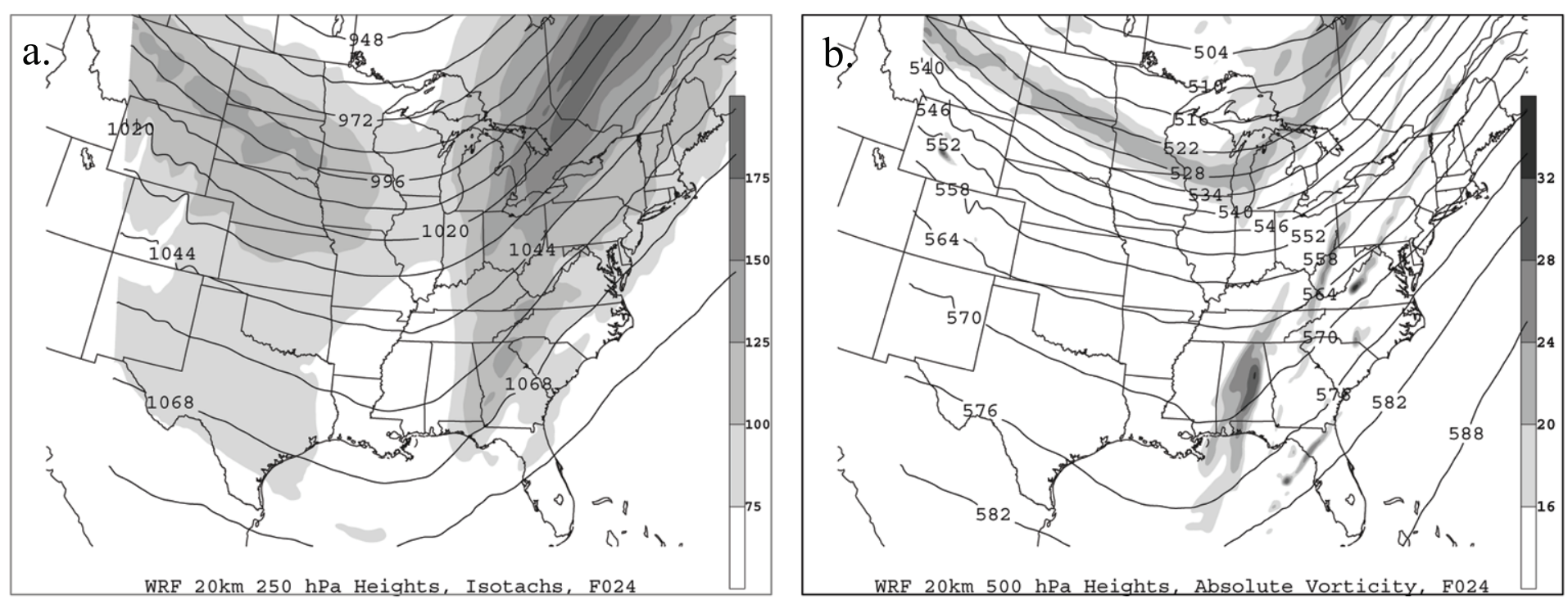

$\bar{u}$
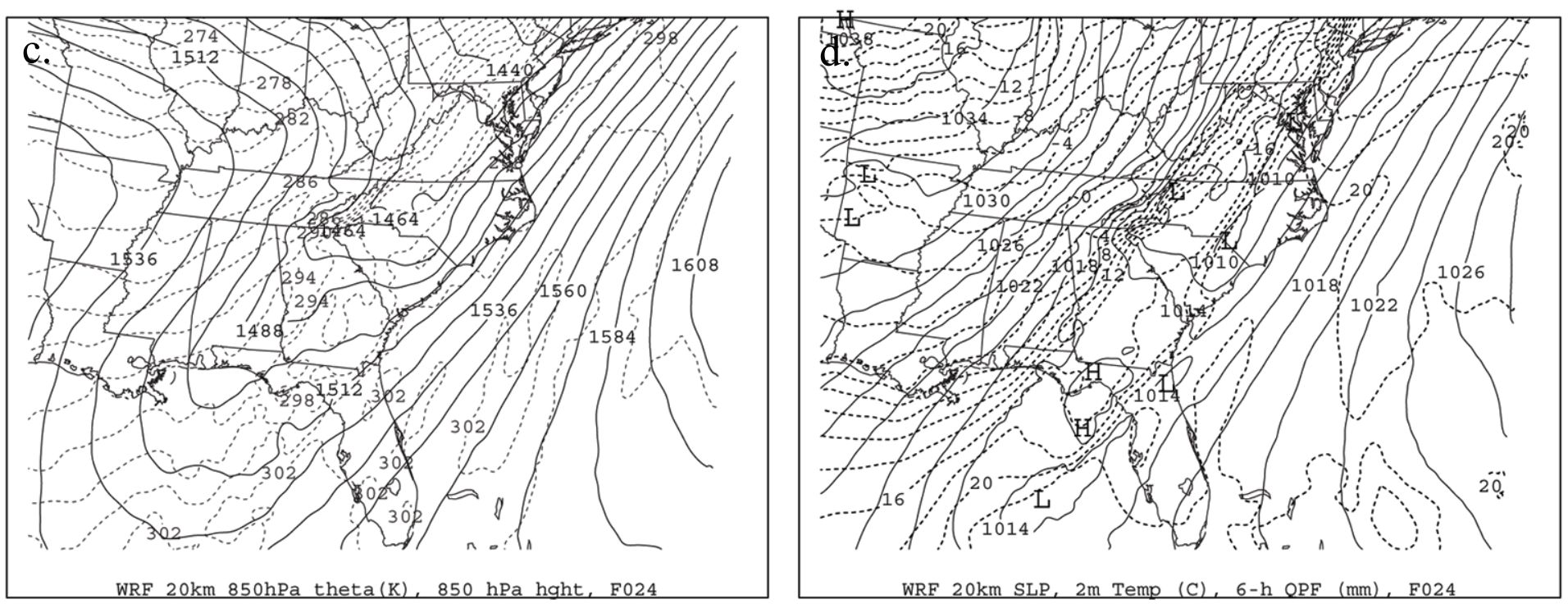

Figure 4.30. As in Fig. 4.29 except for 12 UTC 14 Jan. 

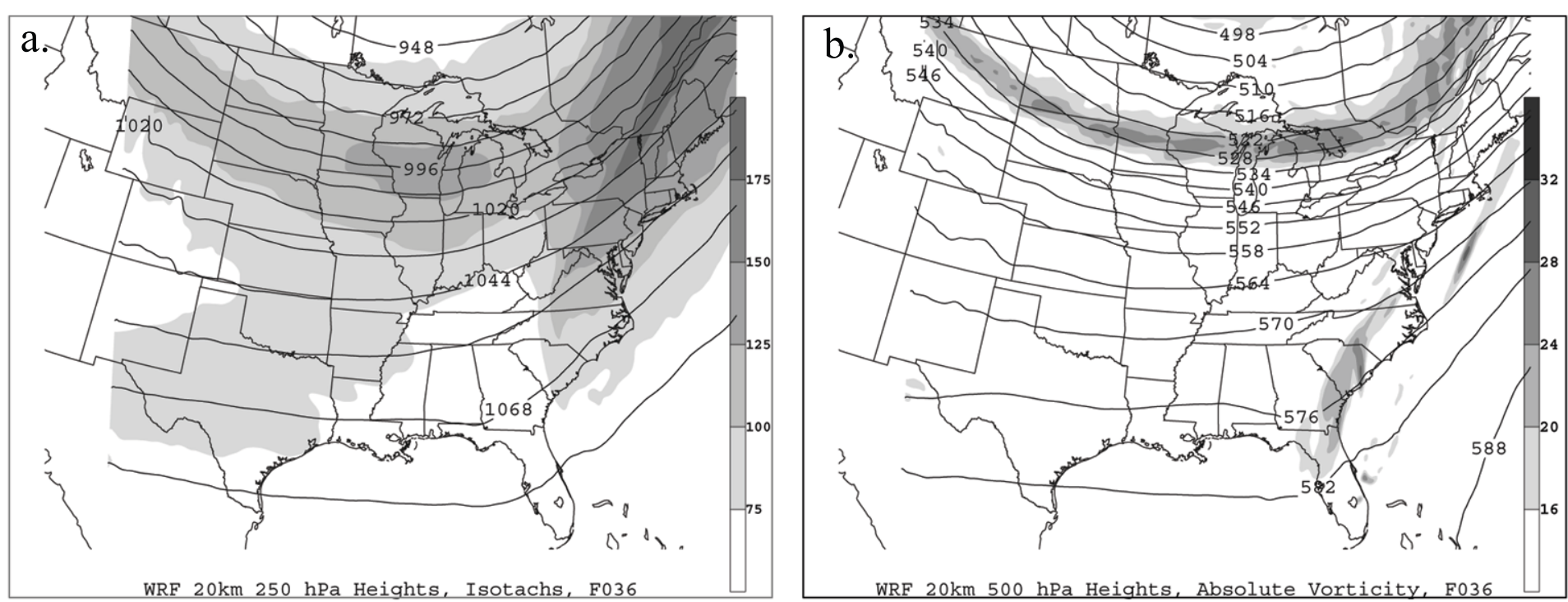

ড̆
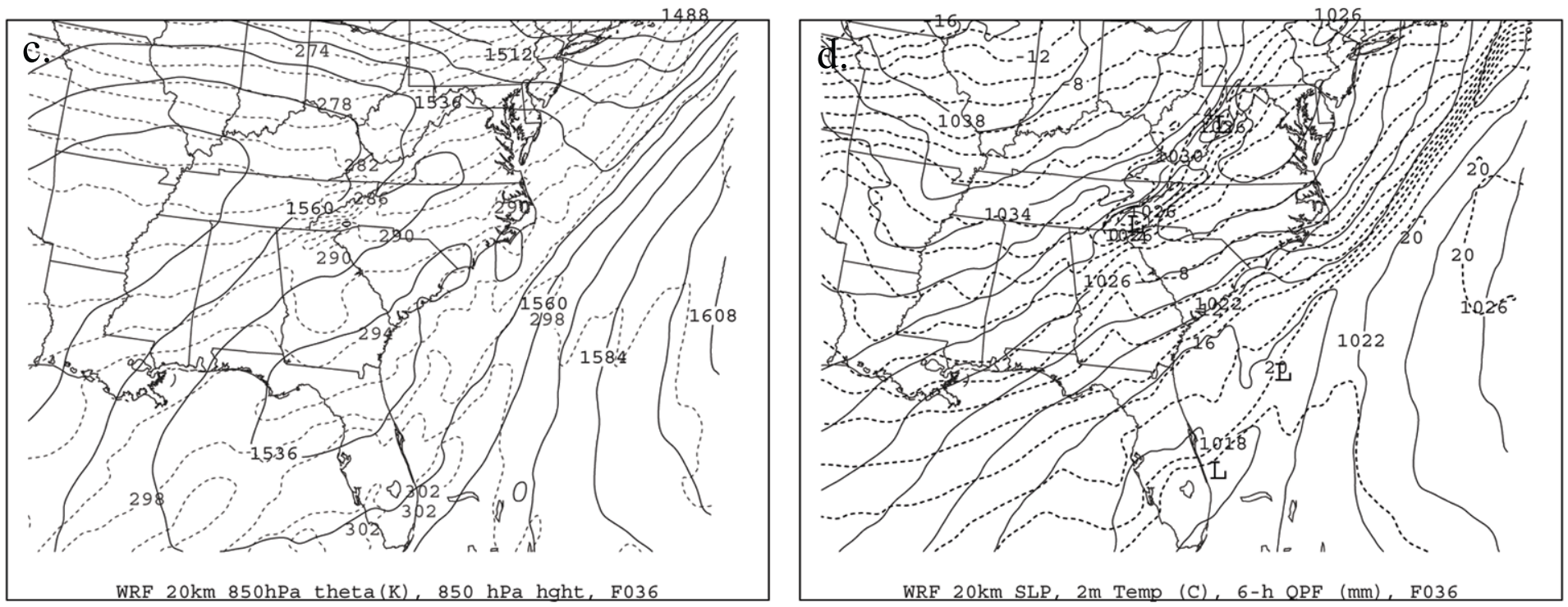

Figure 4.31. As in Fig. 4.29 except for 00 UTC 15 Jan. 

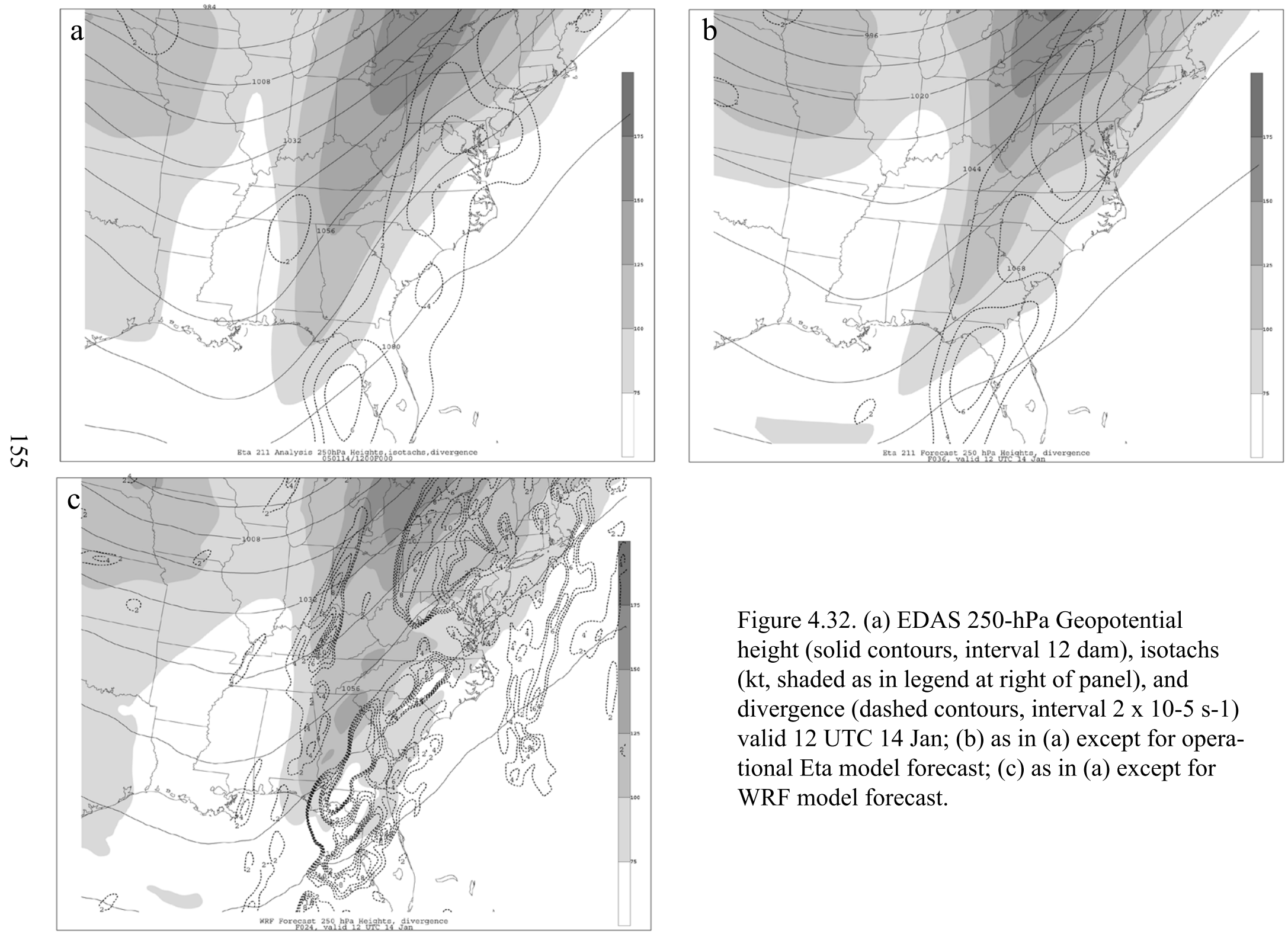

Figure 4.32. (a) EDAS 250-hPa Geopotential height (solid contours, interval 12 dam), isotachs (kt, shaded as in legend at right of panel), and divergence (dashed contours, interval 2 x 10-5 s-1) valid 12 UTC 14 Jan; (b) as in (a) except for operational Eta model forecast; (c) as in (a) except for WRF model forecast. 


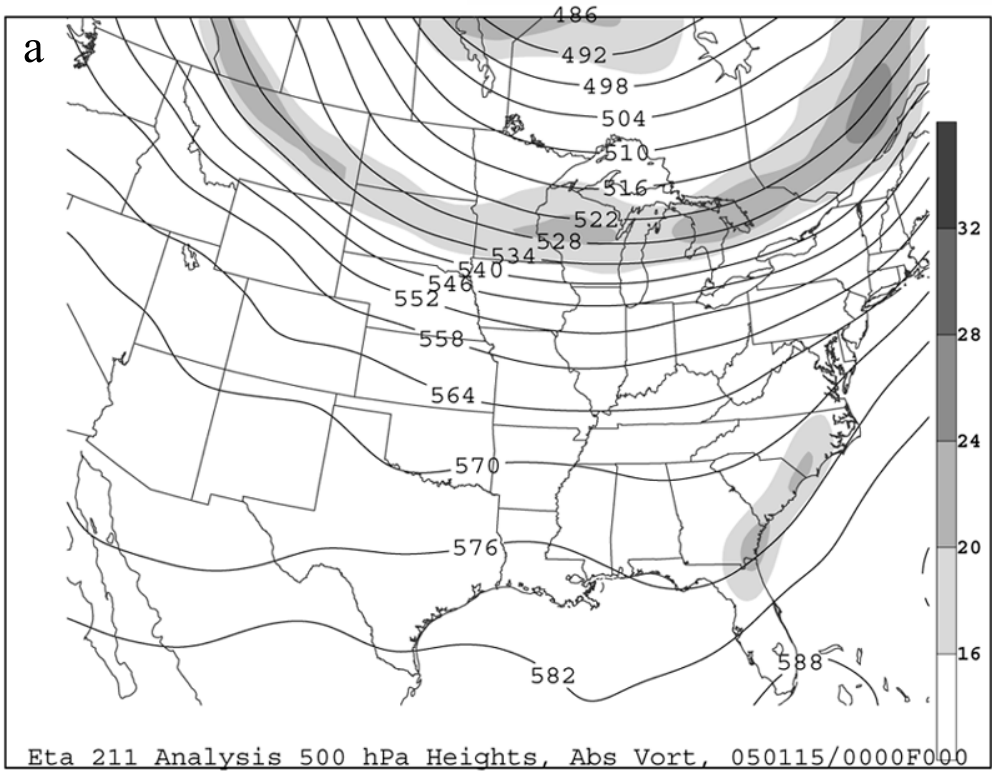

й

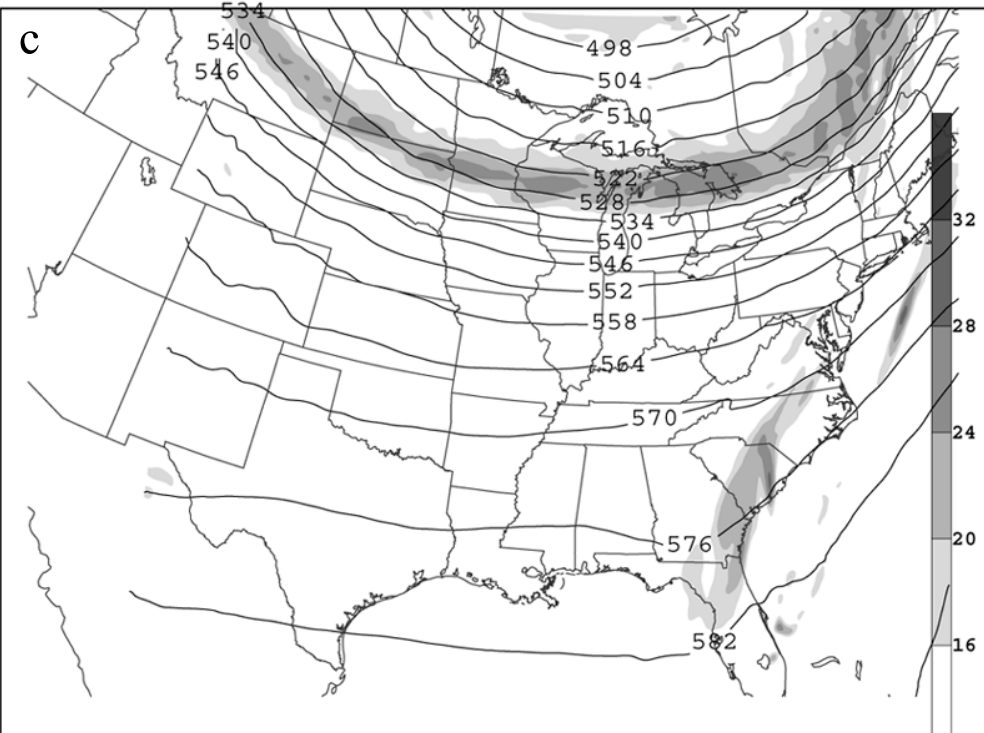

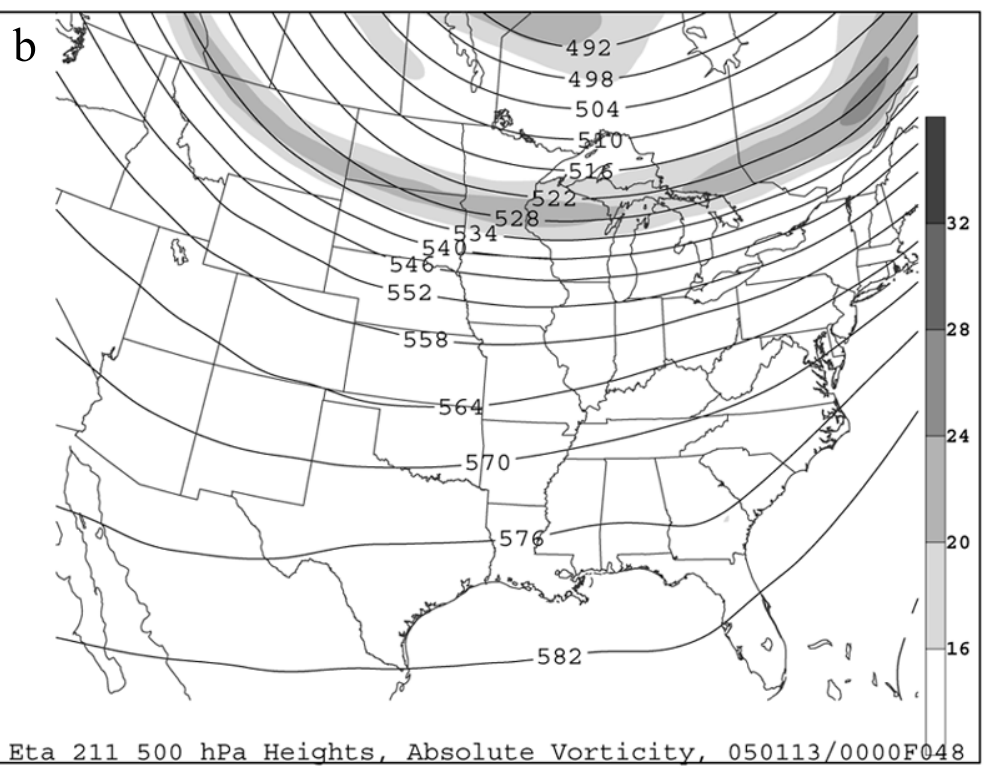

Figure 4.33. (a) EDAS 500-hPa Geopotential height (solid, interval 6 dam) and vorticity (, shaded as in legend at right of panel) valid 12 UTC 14 Jan; (b) as in (a) except for operational Eta model forecast; (c) as in (a) except for WRF model forecast. 

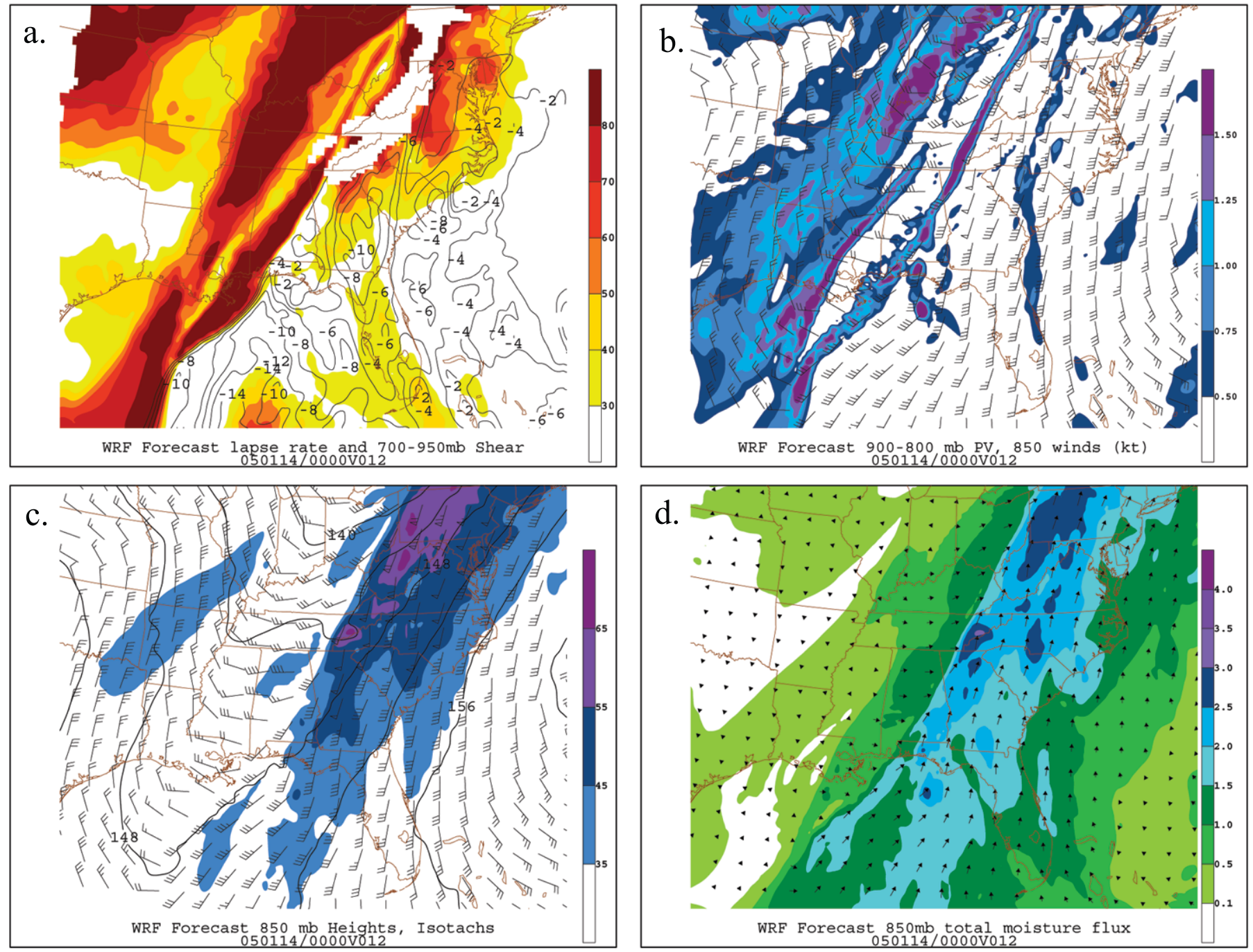

Figure 4.34. WRF 12-h forecast valid 00 UTC 14 Jan 2005: (a) 500-950-hPa equivalent potential temperature difference (solid contours, interval $2 \mathrm{~K}$, positive values omitted) and 700-950-hPa wind shear (10 kt/m, shaded as in legend at right of panel); (b) 900-800 layer-averaged PV (PVU, shaded as in legend at right of panel) and 850-hPa winds (kt, barbs); (c) 850-hPa Geopotential height (solid, interval 4 dam), isotachs (kt, shaded as in legend at right of panel), and 850-hPa winds (kt, barbs); (d) $850-\mathrm{hPa}$ moisture flux ( $\mathrm{g} \mathrm{kg}-1 \mathrm{~m} \mathrm{~s}-1$, shaded as in legend at right of panel) and moisture flux vectors (arrows). 

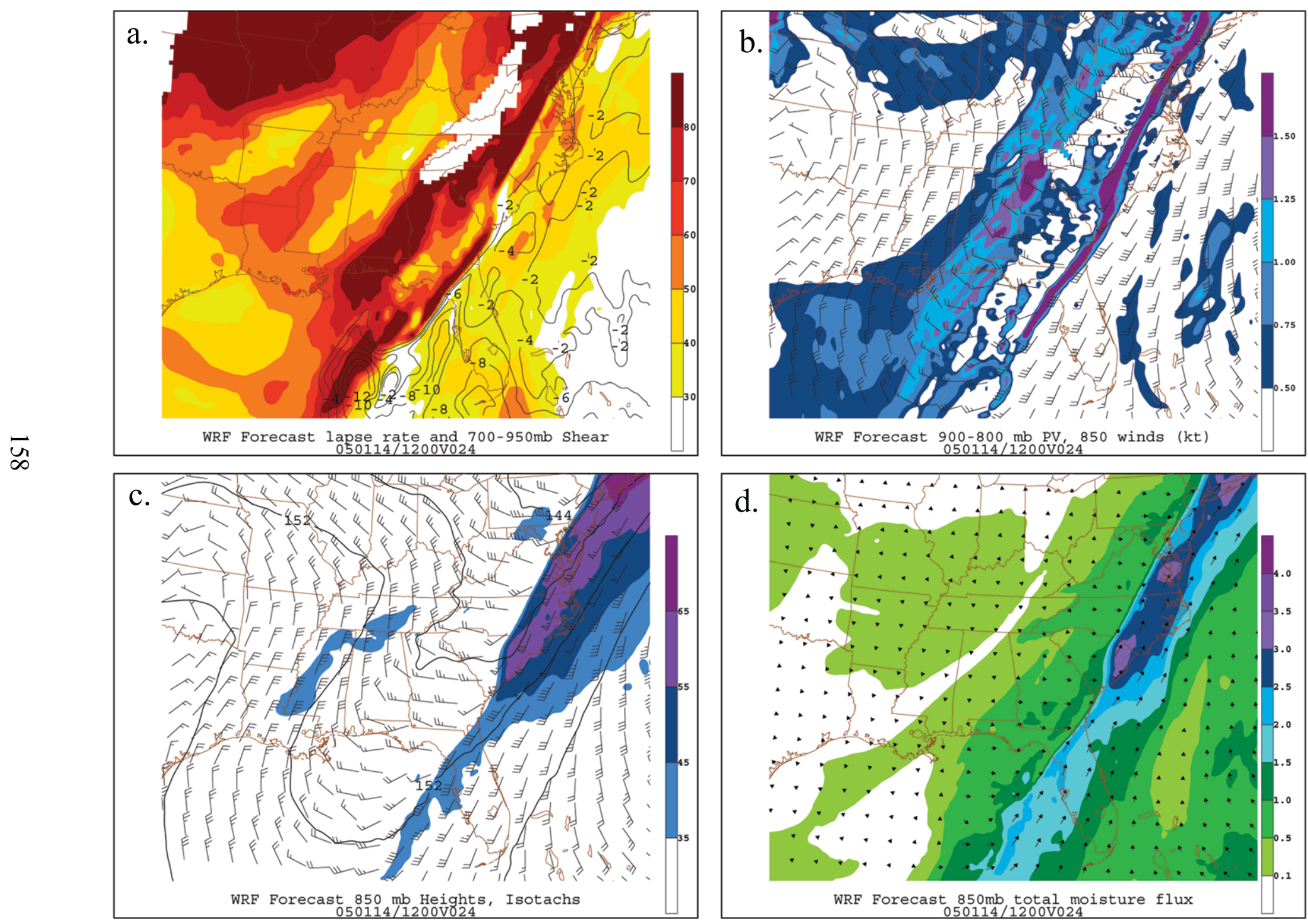

Figure 4.35. As in Fig. 4.34 except for 12 UTC 14 Jan. 

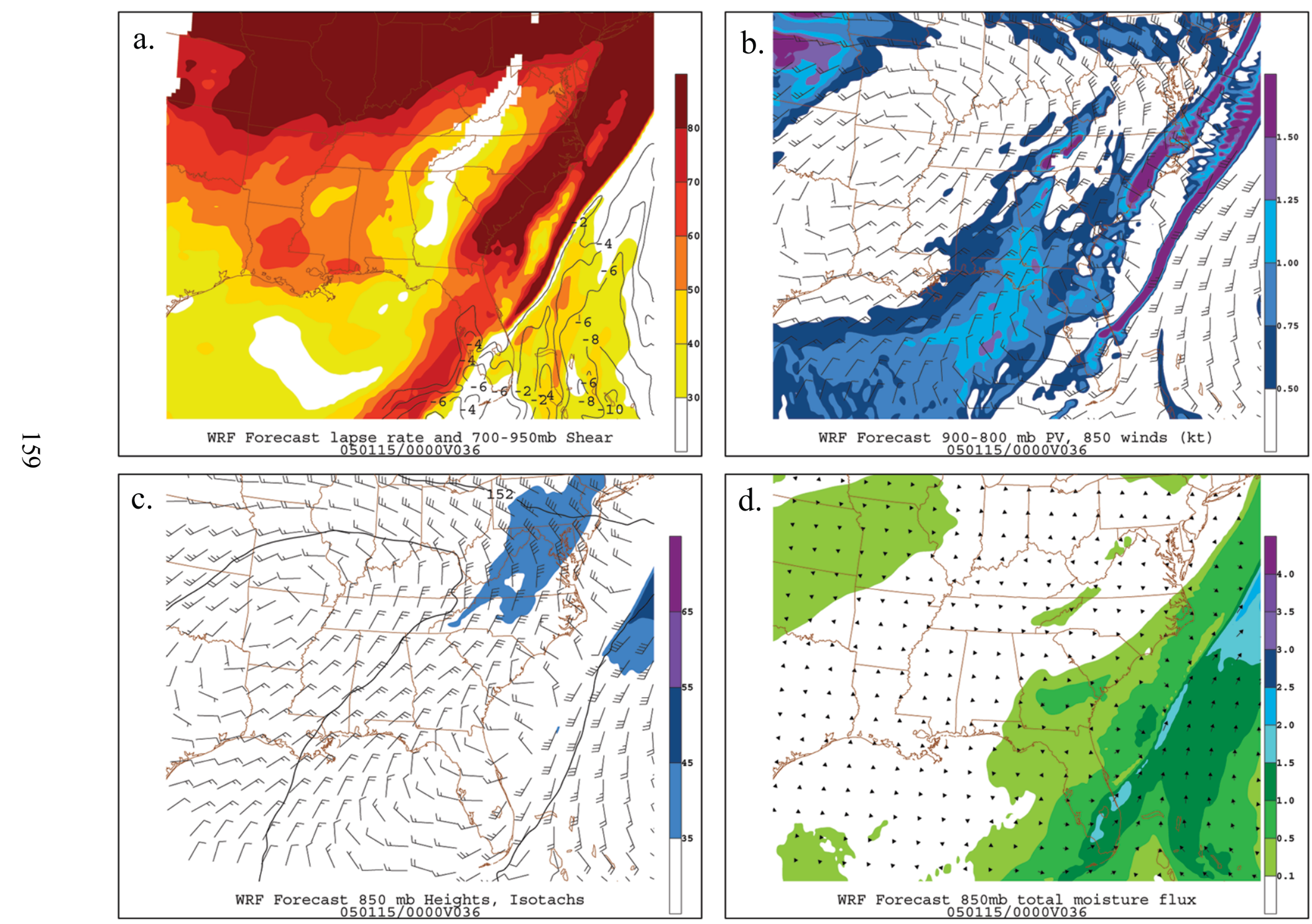

Figure 4.36. As in Fig. 4.34 except for 00 UTC 15 Jan. 

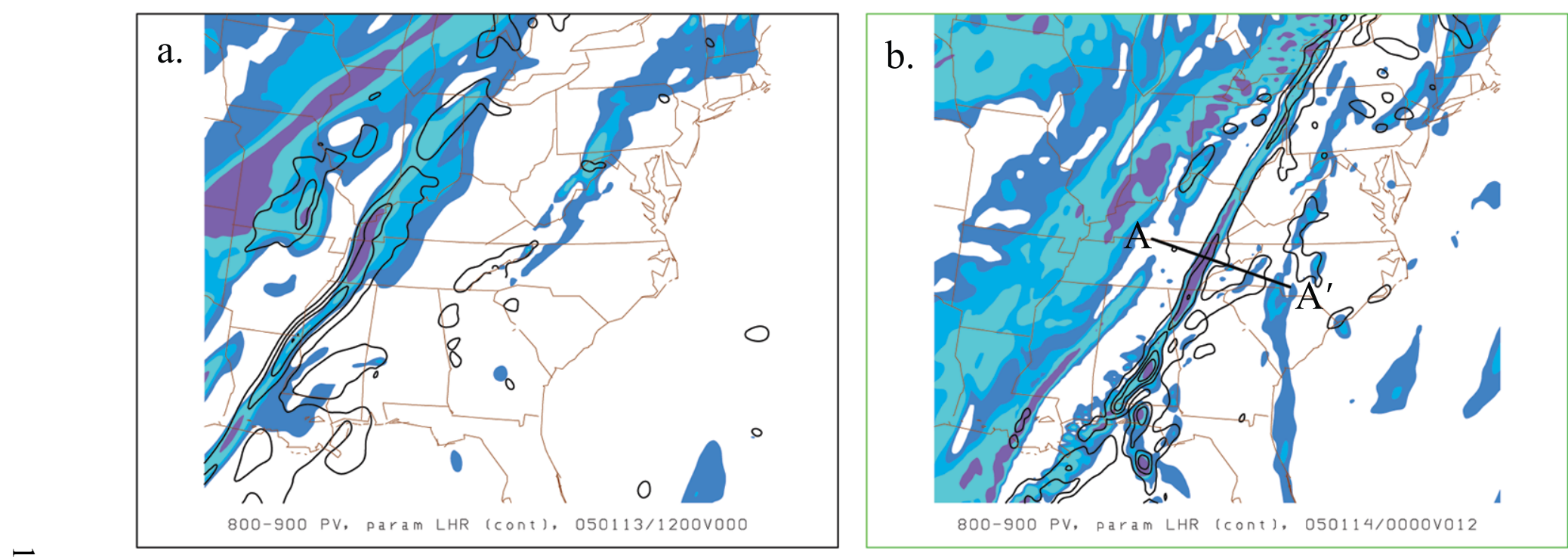

子

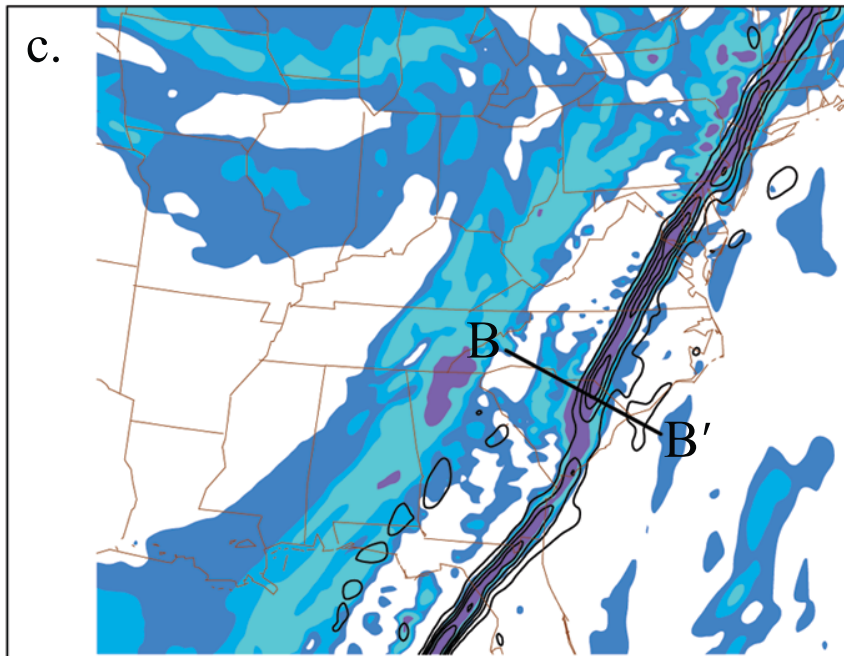

800-900 PV, param LHR (cont), 050114/1200V024

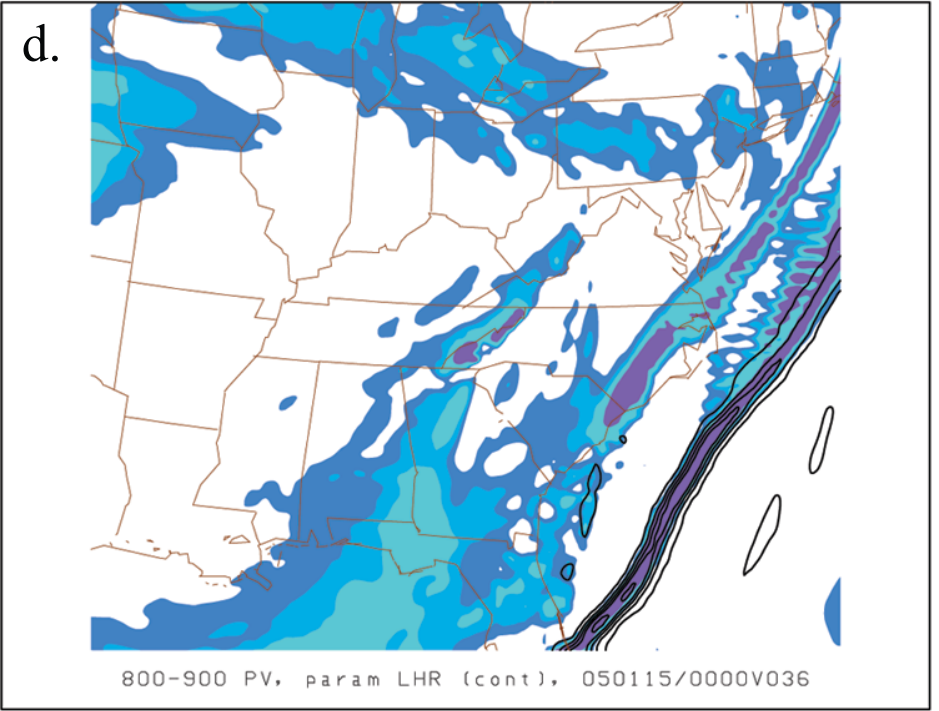

Figure 4.37. (a) 800-900-hPa PV (PVU, shaded as in legend at right of panel) and 850-hPa parameterized latent heating (solid contours, K day-1) for 12-h forecast valid 12 UTC $13 \mathrm{Jan}$; (b) as in (a) except for 12-h forecast valid 00 UTC 14 Jan; (c) as in (a) except for 24-h forecast valid 12 UTC 14 Jan; (d) as in (a) except for 36$\mathrm{h}$ forecast valid 00 UTC 15 Jan. Solid line A-A' in (b) denotes cross-section shown in Fig. 4.40 and solid line B-B' in (c) denotes cross-section shown in Fig. 4.41. 
a.

$\sqrt{2}$

c.

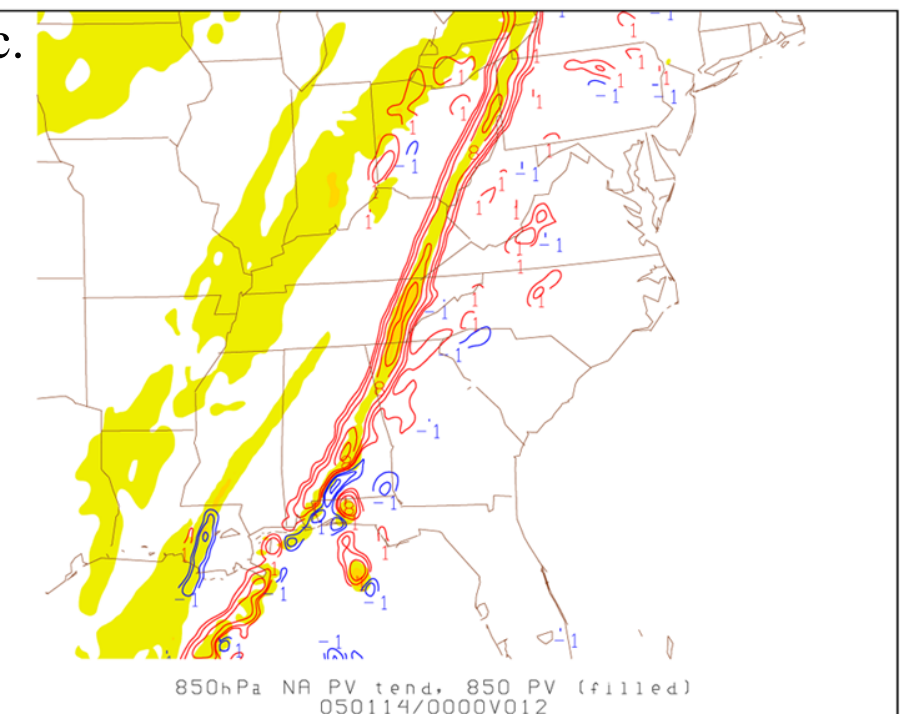

b.

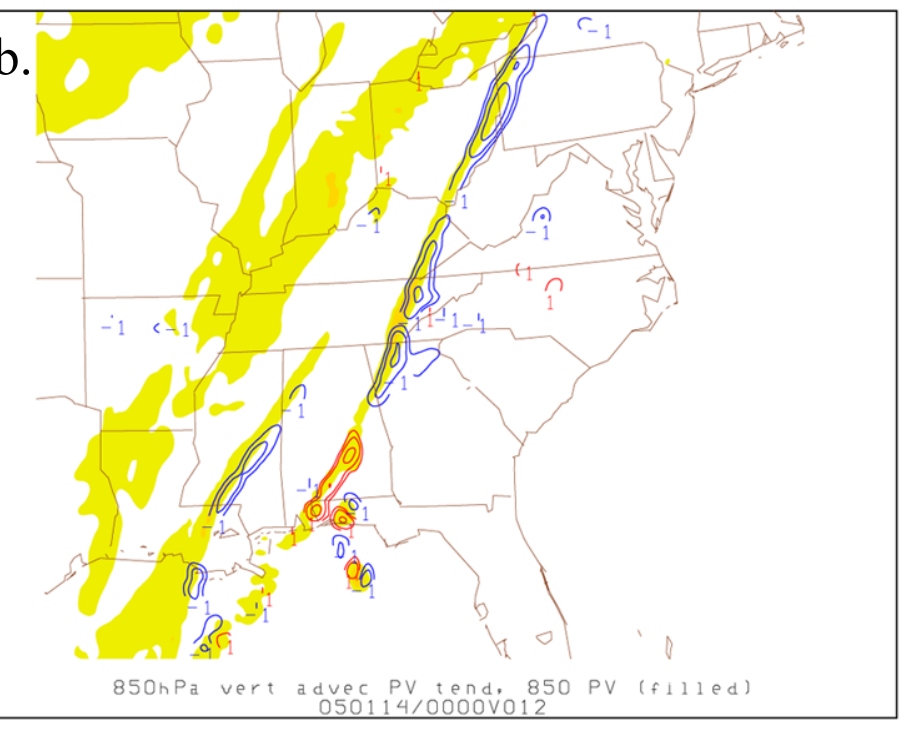

d.

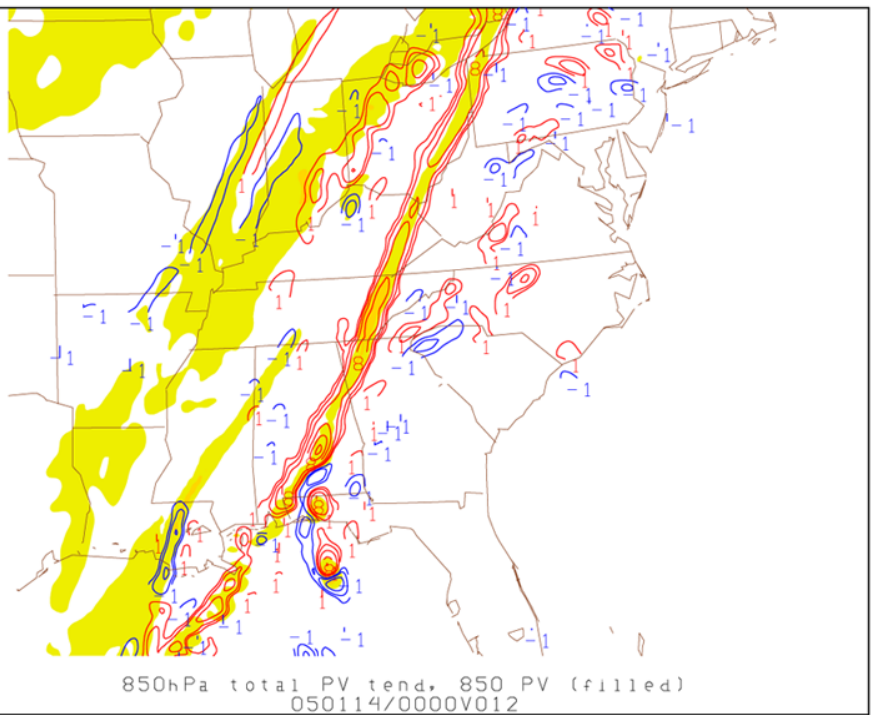

Figure 4.38. PV budget summary for 00 UTC 14 Jan: (a) 800-900-hPa PV (PVU, shaded as in legend at right of panel) and 850-hPa horizontal advective PV tendency (contour interval 1 PVU day-1, red (blue) contours correspond to positive (negative) values); (b) as in (a) except for the vertical advective PV tendency; (c) as in (a) except for nonadvective PV tendency; (d) as in (a) except for total PV tendency. 

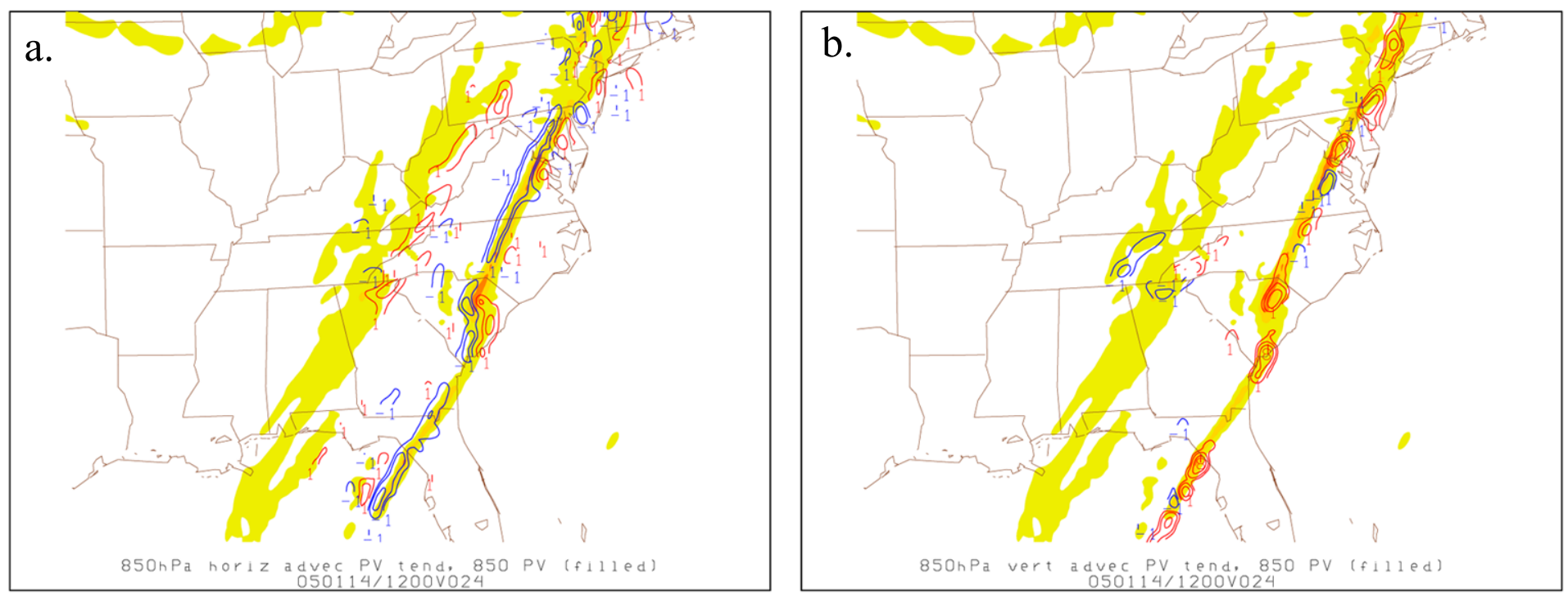

ì
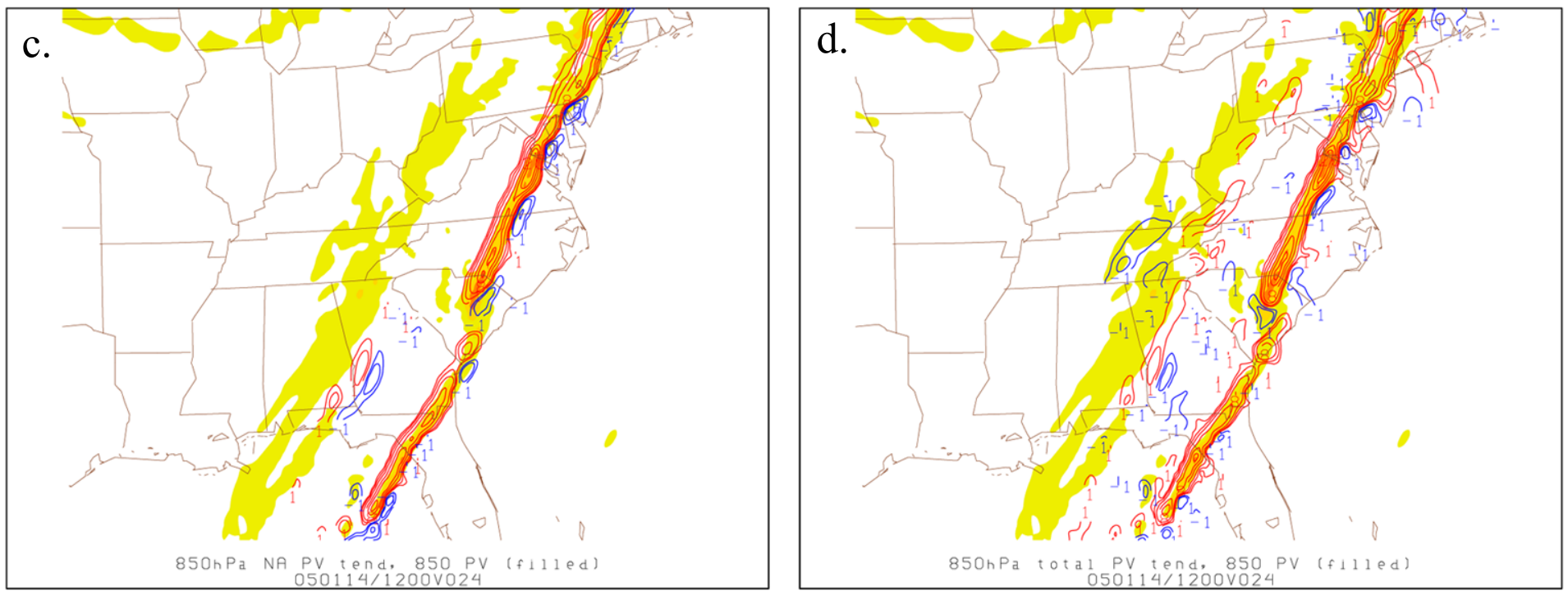

Figure 4.39. As in Fig. 38 except for 12 UTC 14 Jan. 


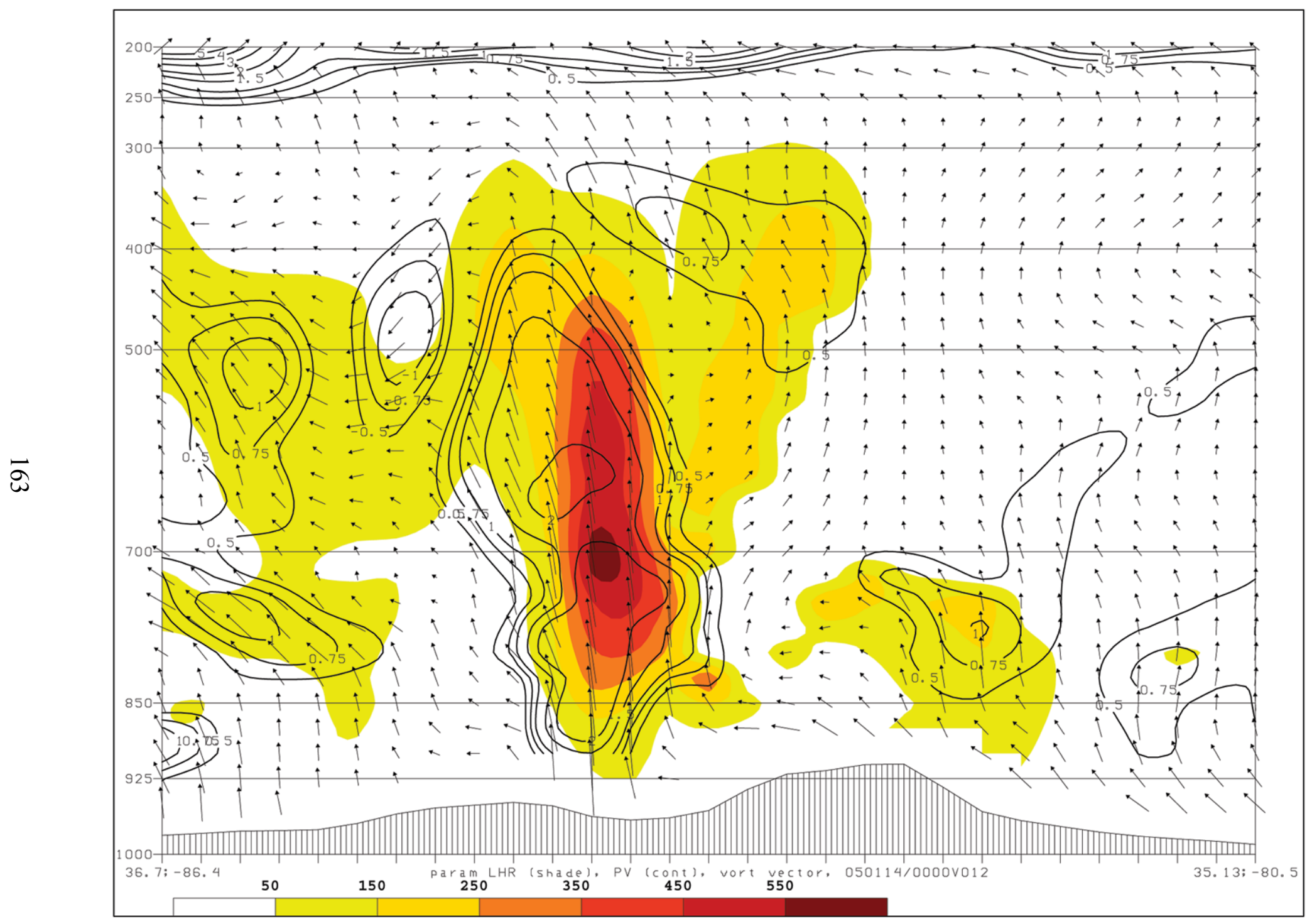

Figure 4.40. Cross section taken along line A-A' in Fig. 4.37b valid at 00 UTC 14 Jan showing parameterized latent heating (K day-1, shaded as in legend at bottom of panel), PV (solid contours, PVU), and absolute vorticity vectors (arrows). 


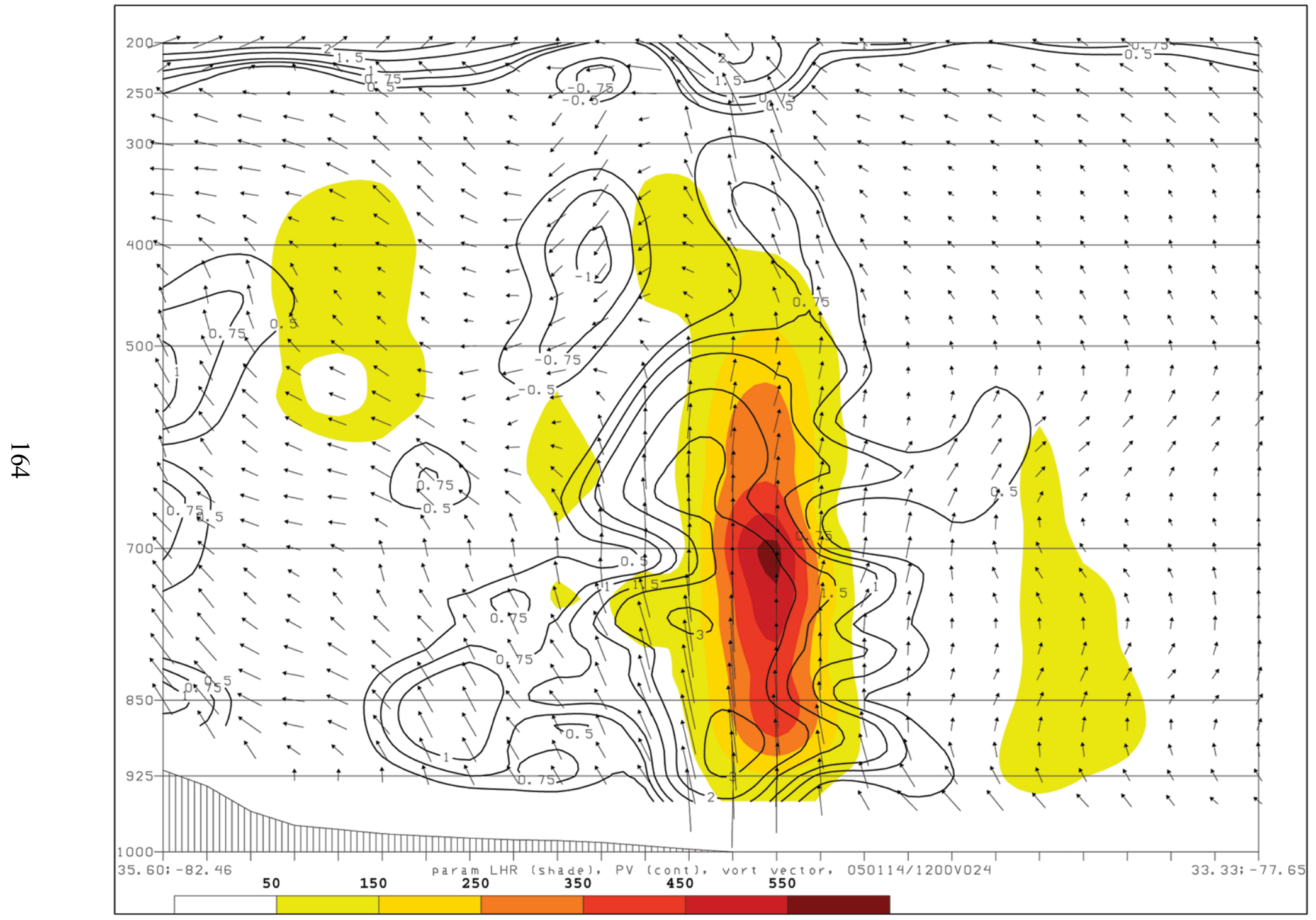

Figure 4.41. As in Fig. 4.40, except cross-section taken along B-B' in Fig. 4.37c. 


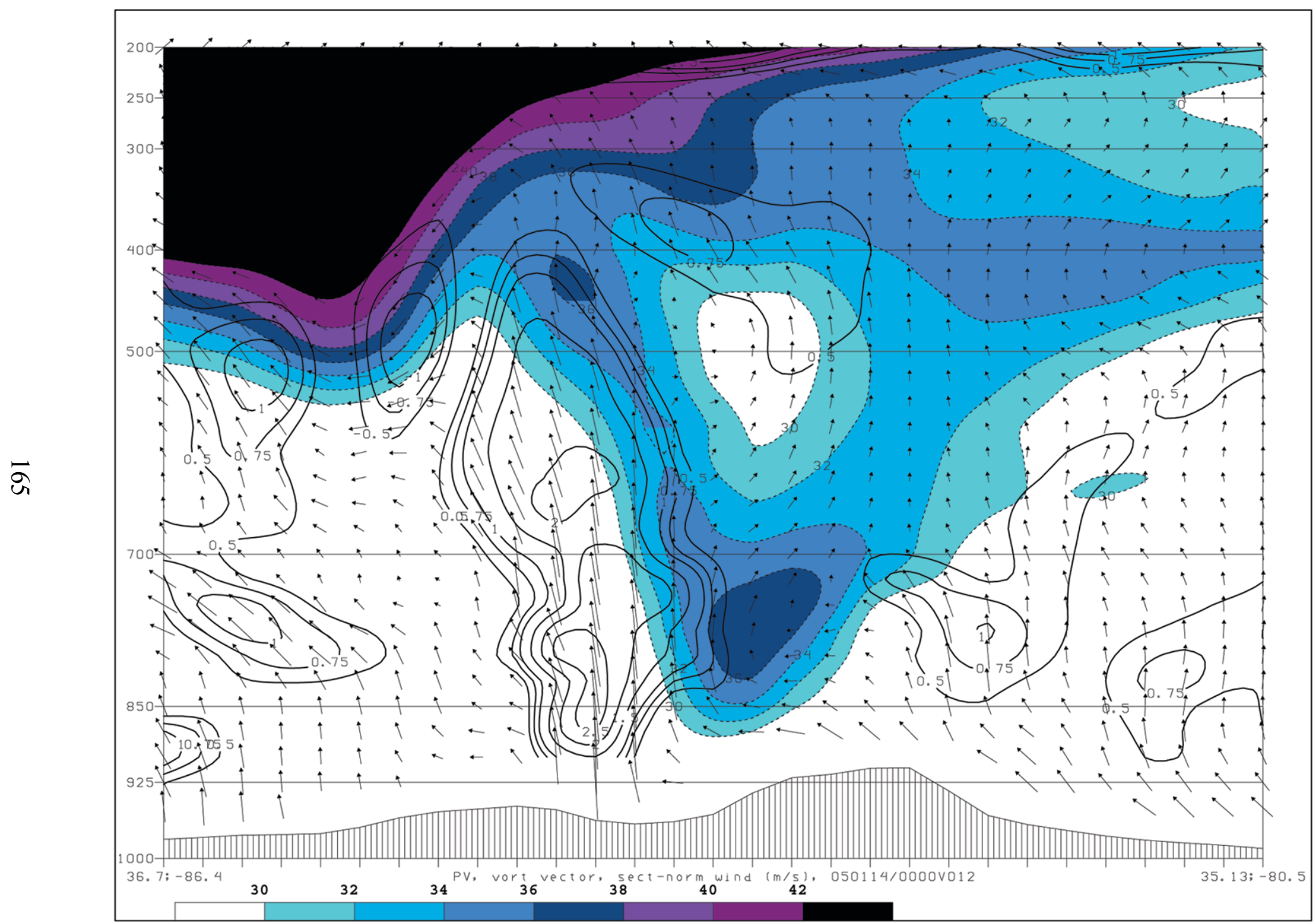

Figure 4.42. Cross section taken along line A-A' in Fig. 4.37b valid at 00 UTC 14 Jan showing PV (solid contours, PVU), absolute vorticity vectors (arrows), and section-normal wind speed (kt, shaded as in legend at bottom of panel). 


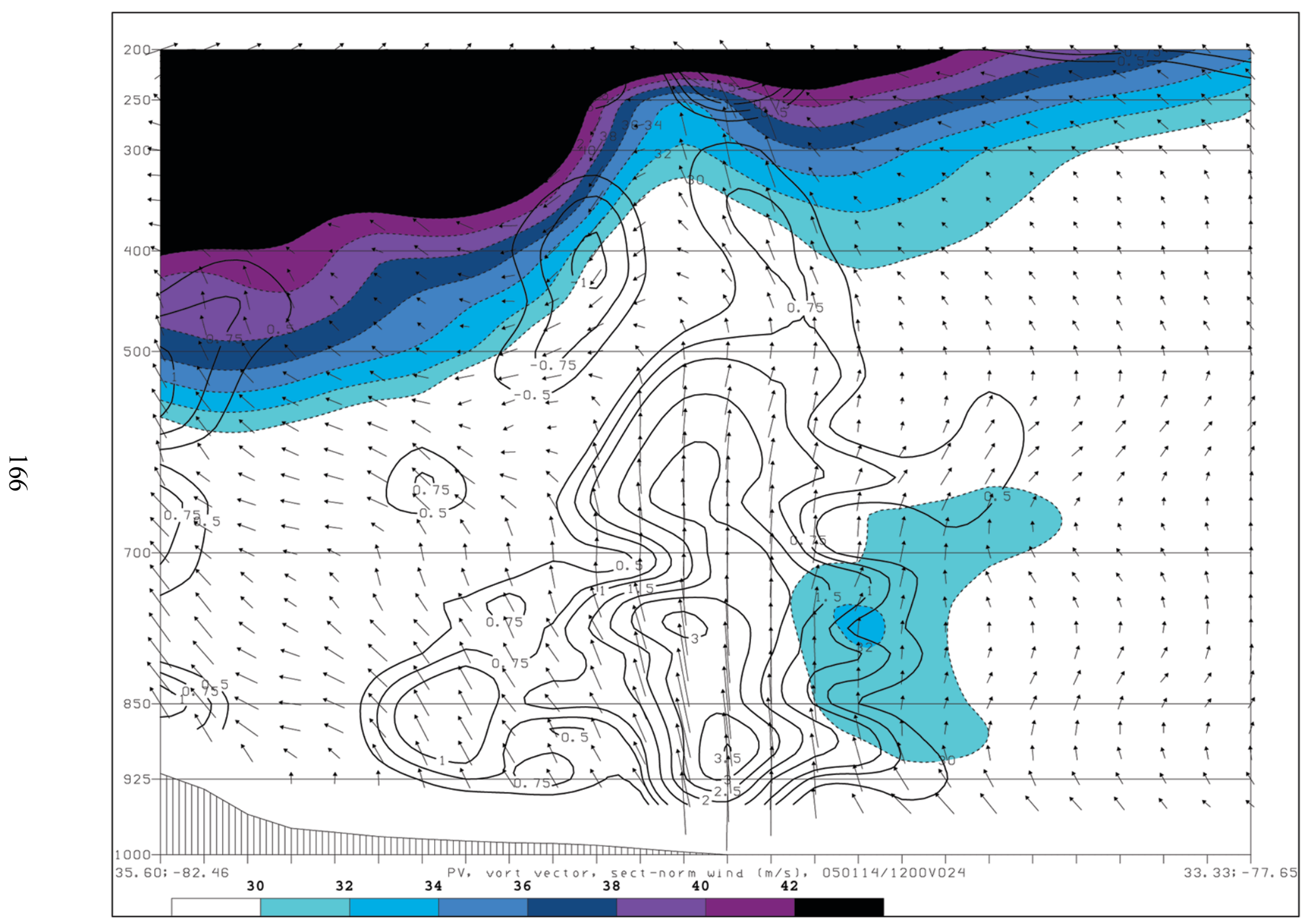

Figure 4.43. As in Fig. 4.42, except cross-section taken along B-B' in Fig. 4.37c. 

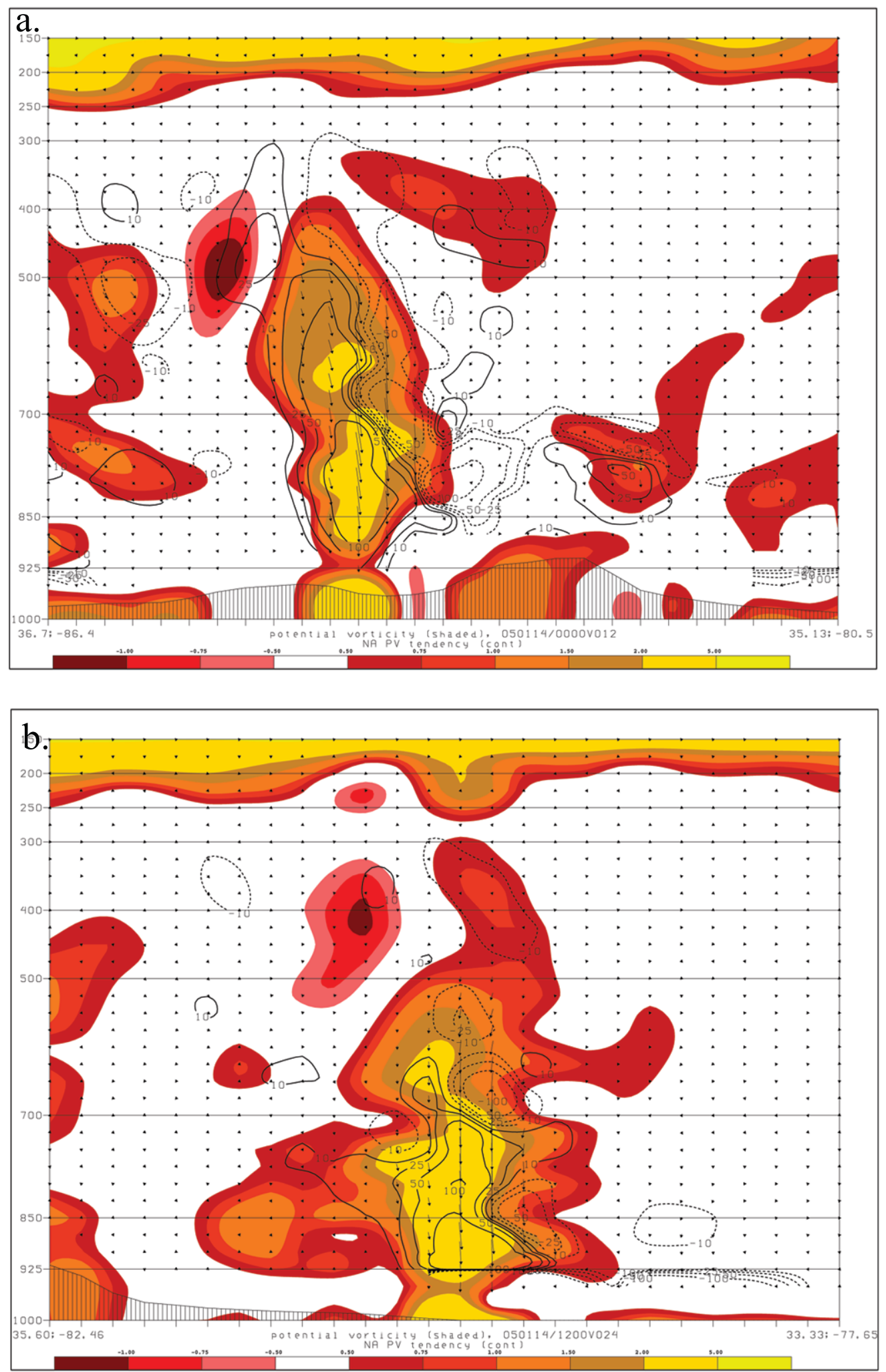

Figure 4.44. (a) Cross section taken along line A-A' in Fig. 4.37b valid at 00 UTC 14 Jan showing PV (PVU, shaded as in legend at bottom of panel), non-advective PV tendency (contour interval 1 PVU day-1, solid (dashed) lines denote positive (negative) values), and non-advective PV flux vectors (arrows); (b) as in (a) except cross-section taken along B-B' in Fig. 4.37c. 

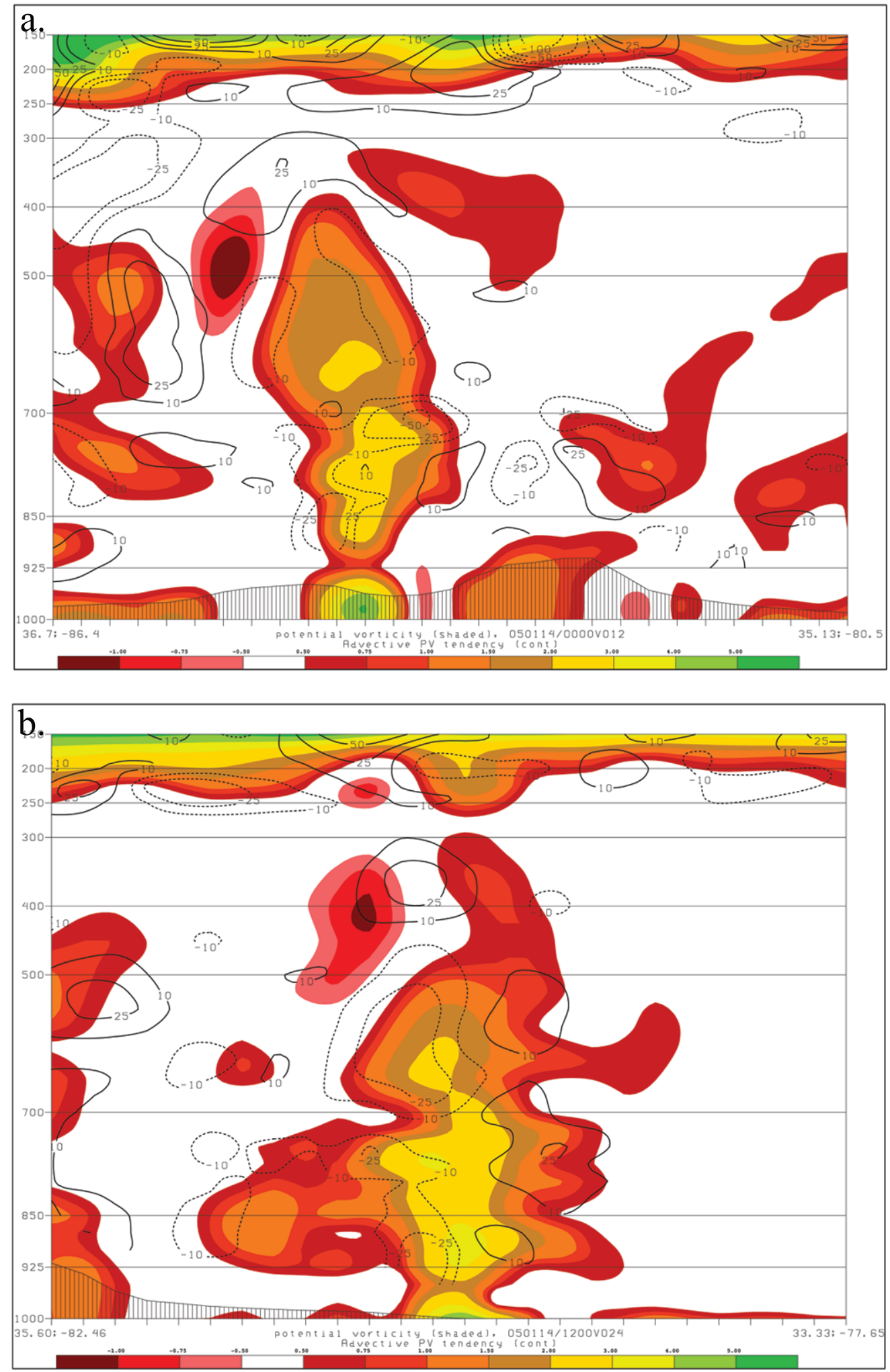

Figure 4.45. As in Fig. 4.44 except for advective PV tendency. 

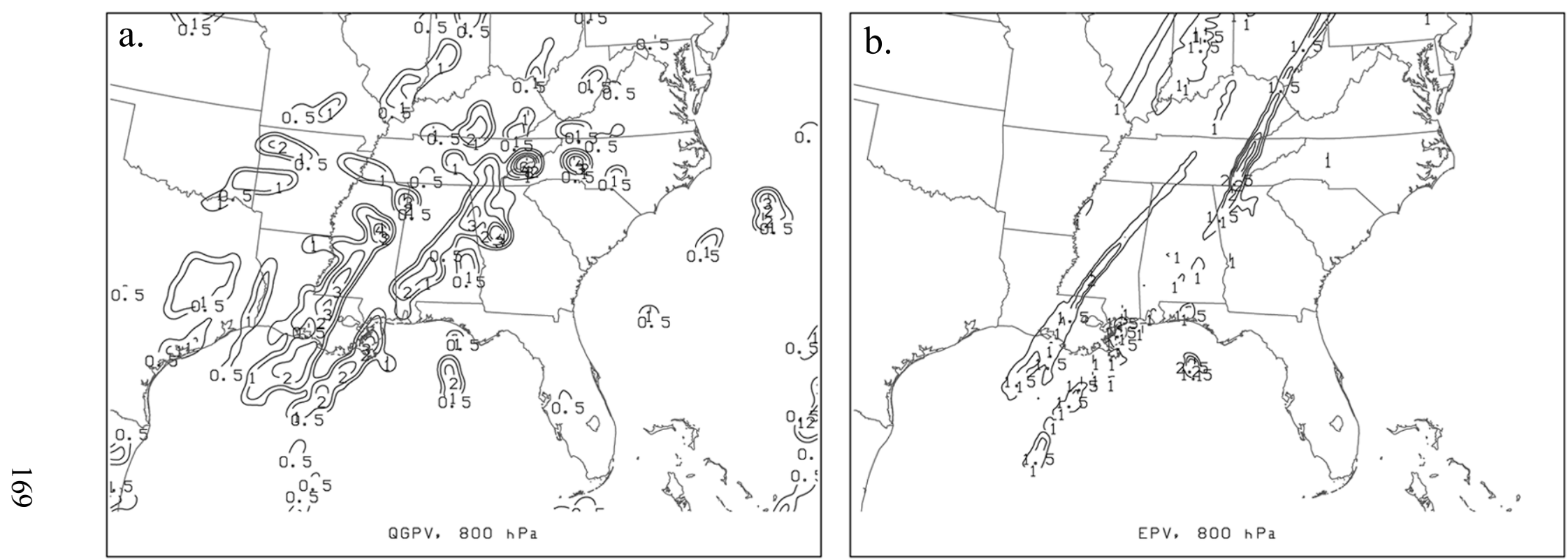

Figure 4.46. (a) QGPV (solid, contour interval 4 x 10-4 s-1) valid 00 UTC 14 Jan; (b) as in (a) except EPV (solid, contour interval .5 PVU, values below 1 PVU omitted). 

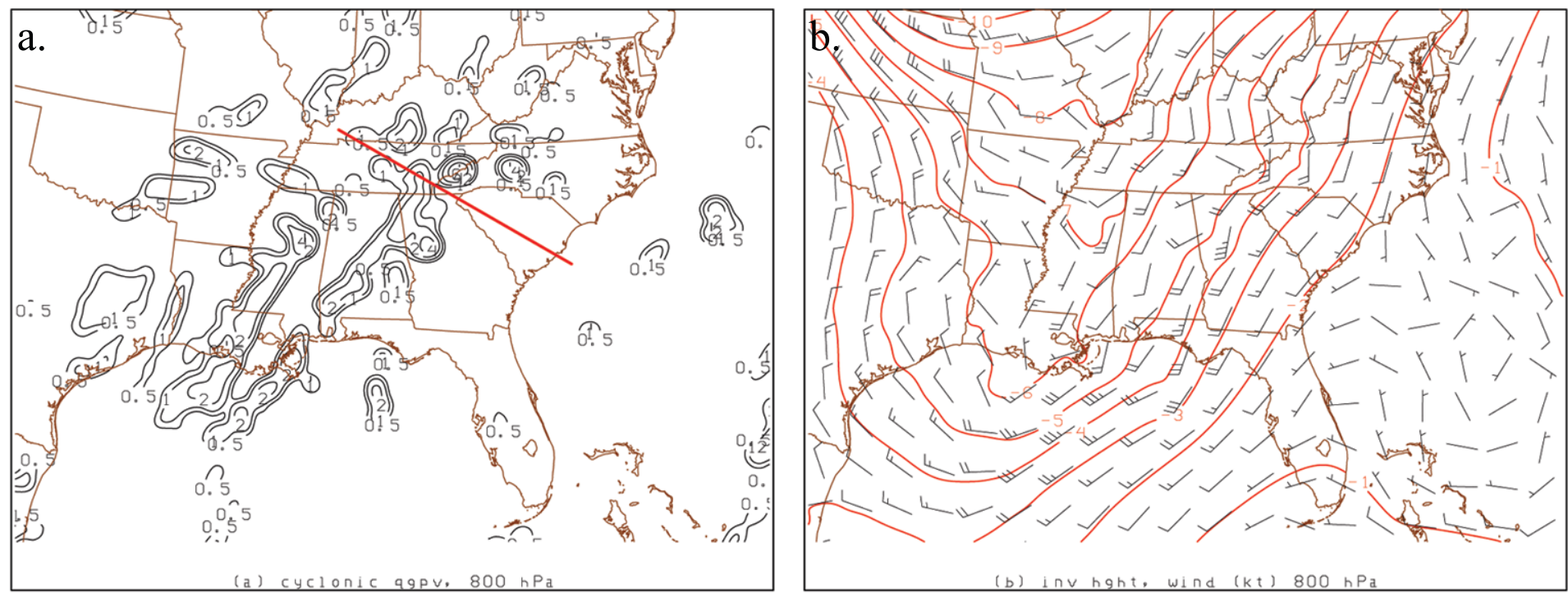

$\breve{ఠ}$
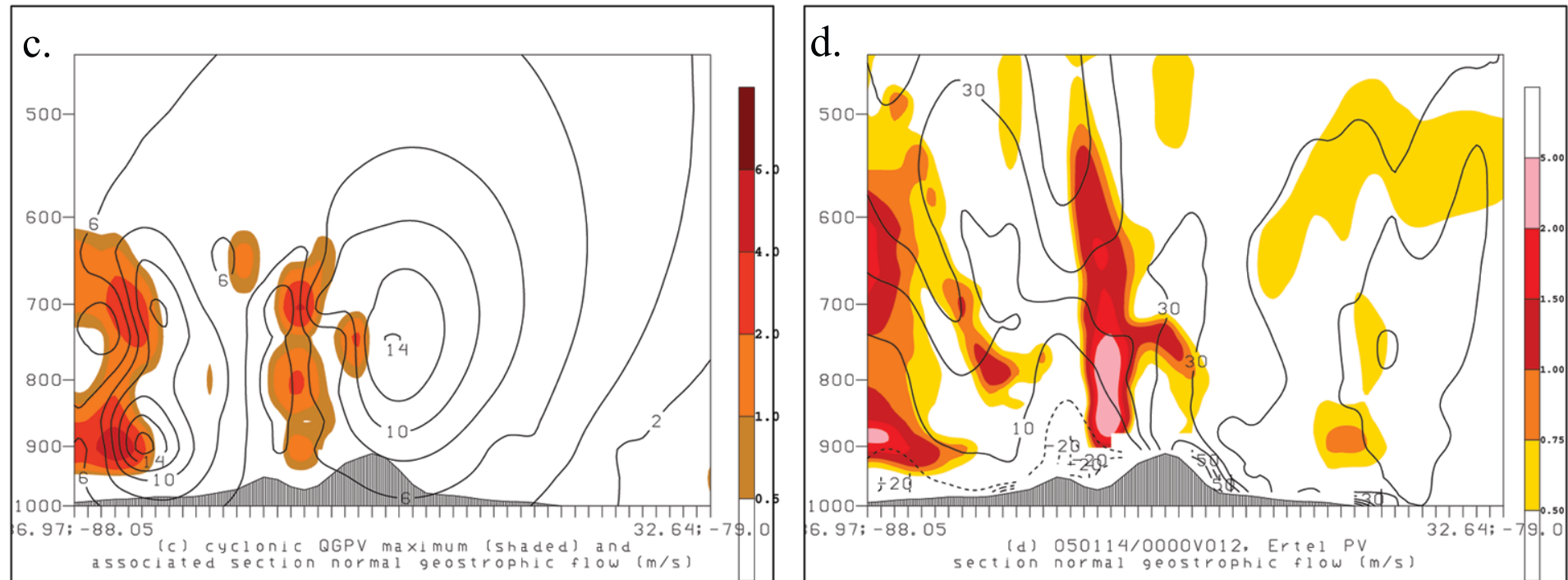

Figure 4.47. Inversion summary for cyclonic QGPV anomaly at 00 UTC 14 Jan: (a) cyclonic QGPV anomaly at $900 \mathrm{hPa}$ (solid, contour interval 4 x 10-4 s-1); (b) geopotential height (solid, contour interval $400 \mathrm{~m}$ ) and geostrophic wind recovered from inversion of cyclonic QGPV anomaly; (c) cross section of QGPV and section-normal geostrophic wind component (m s-1, solid (dashed), positive (negative) for flow into (out of) section); (d) as in (c) except for Ertel PV and observed section-normal geostrophic wind speed. 

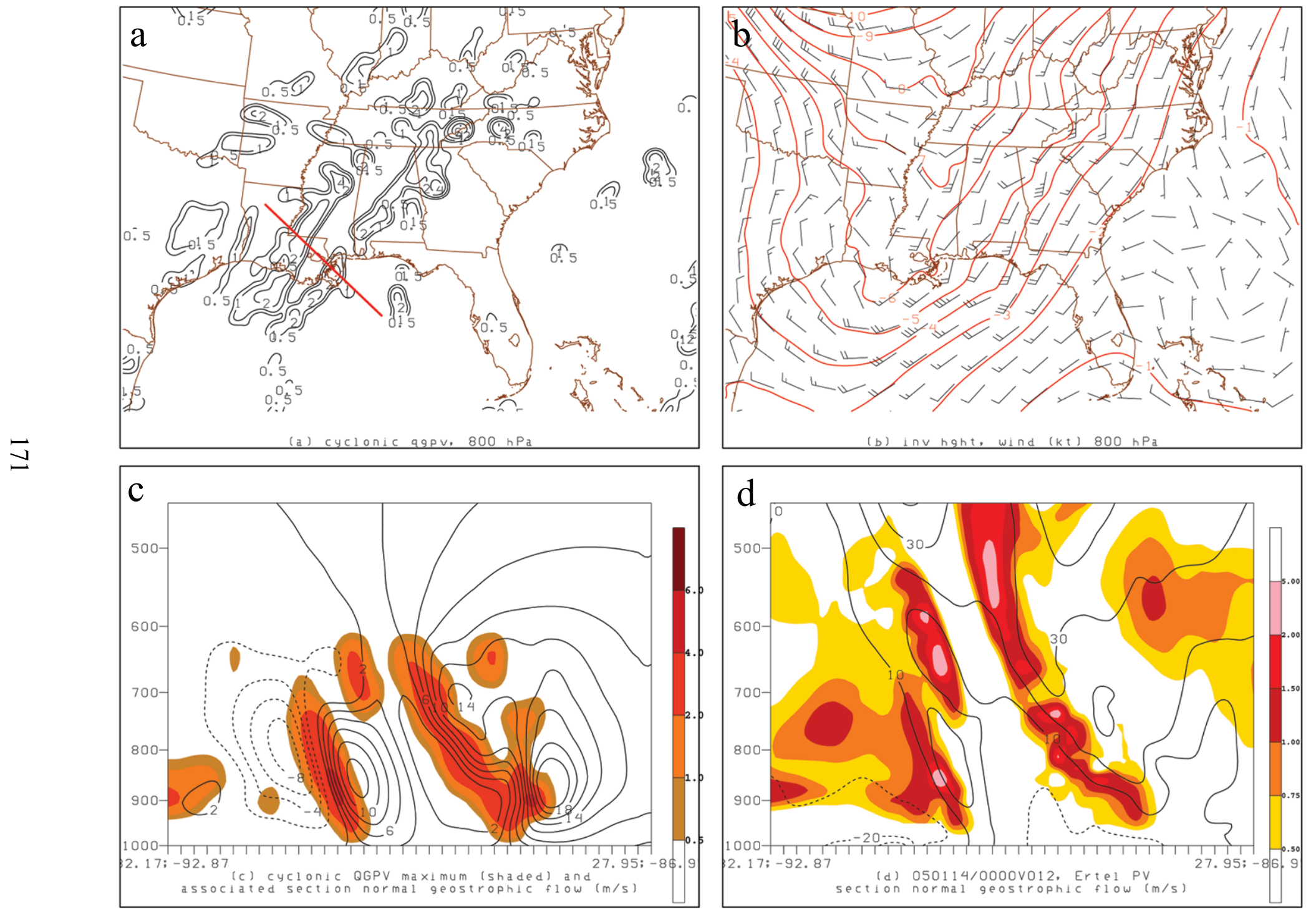

Fig. 4.48. As in Fig. 4.47 except cross-section taken along line marked in panel (a) 


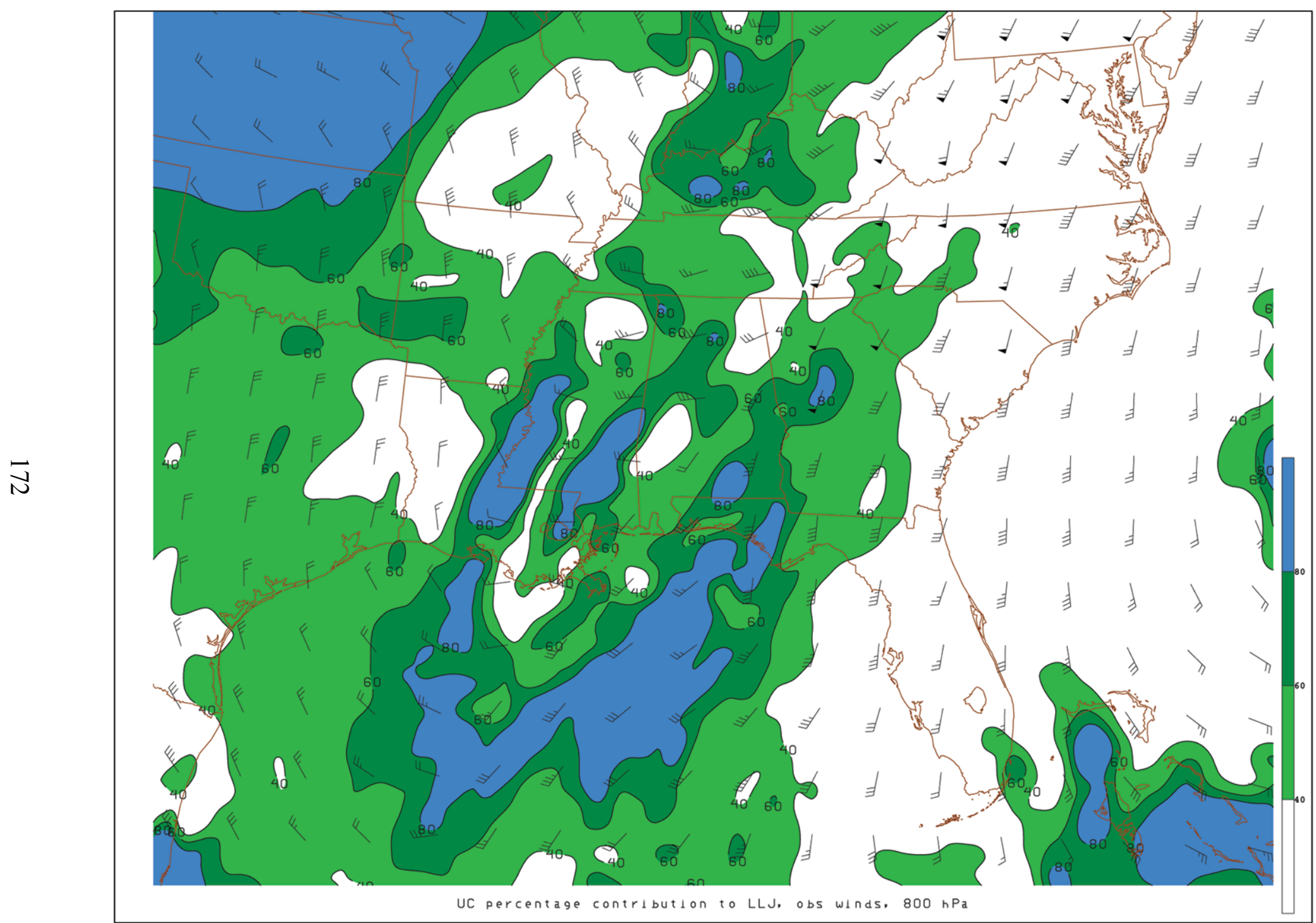

Figure 4.49. Ratio of inverted LLJ (inverted 800-hPa winds) to total LLJ (total 800-hPa winds) (percent, shaded as in legend at right) and $800-\mathrm{hPa}$ total flow (barbs, $\mathrm{kt}$ ). 


\section{Discussion, Applications, and Conclusions}

\subsection{Discussion}

\subsubsection{Scenario 3 and the continuum of UC environments}

As explained in Chapter 2, the original S3 hypothesis was altered after the Eta QPF error climatology showed that few large QPF errors were associated with such setups (over the relatively brief time period examined). While setups similar to the S3 schematic (Fig. 1.8) are encountered with some degree of frequency, they often transition into environments that more closely resemble $\mathrm{S} 1$. Therefore, $\mathrm{S} 3$ is considered to be an "early" scenario, and is just one example of an UC environment that does not fit neatly into a S1 or S2 category. Several examples of events that initially resembled the S3 schematic were examined, and brief descriptions of two S3 events are given in the Appendix.

The simplicity of the S1 and S2 labels are also their potential limitation; very rarely does an UC situation look exactly like one of scenarios depicted by the simplistic schematics. In fact, even the case studies chosen here are not perfect representations of those scenario schematics; rather they were the best examples that could be found using the brief climatology.

Instead of labeling UC events definitively as $\mathrm{S} 1$ or $\mathrm{S} 2$, it is probably more realistic to consider a continuum of scenarios, with S1 and S2 defining the two extremes. Likewise, the downstream precipitation patterns also fall into a similar continuum. For example, the faster the $\mathrm{UC}$ is moving relative to the main system, and the more it resembles S1, then the more the downstream QPF will likely have to be reduced. 
Conversely, the more slowly the UC is moving relative to the main system, and the more it resembles the S2 schematic, the more likely it is that downstream precipitation will be enhanced.

However, the best way to evaluate $\mathrm{UC}$ cases is to focus on the physical processes that have been found to define the different scenarios. Doing so will likely prevent the problems associated with over-simplified case "labeling." While it will take time and operational practice to identify how best to pinpoint where on the UC continuum a realtime event falls, several guidelines and possibilities are discussed in the following sections.

\subsubsection{PV budget comparisons}

The results of the Ertel PV budgets performed on the S1 and S2 case studies are detailed in Chapters 3 and 4. The PV budgets reveal that diabatic processes played an important role in both cases, and that LHR associated with deep UC produced strong diabatic PV anomalies that proved to be useful tracers for the systems in question.

The major outcome of the two PV budgets is the finding that PV tendencies align differently and provide different contributions to the forward motion of each type of system. In S1 it is believed that the combination of advective and nonadvective positive PV tendencies that flank the eastern edge of the diabatic PV tower can be used to explain the faster forward motion that characterizes this scenario. Conversely in S2, the weaker positive PV tendencies (both advective and nonadvective) toward the front of the system can explain the limitation of the speed at which the S2 system moves eastward. 
Describing convective system motion is a complex problem and many processes likely play significant roles in determining overall system motion. Momentum redistribution in convective lines is one process that is currently unaccounted for by the $\mathrm{CP}$ schemes used in operational models. A major complication in using signatures such as the PV diagnostics discussed here as forecasting tools is that for S1 systems, model forecasts employing a CP scheme will not be able to account for the advective tendencies that are hypothesized to at least partially result from momentum transport. The results of the PV investigation performed for each case study support the idea that this process may be crucial to correctly representing MCS motion in numerical model forecasts.

\subsection{Application}

\subsubsection{The nature and importance of UC system motion}

An unexpected result of this study is that the MCS in S1 did not appear to move as much via propagation as was anticipated at the beginning of this investigation. As shown in Chapter 3, PV budget results (as well as an independent check using wind and radar calculations) instead show that the system motion is actually largely advective (though its advected motion is comparatively fast relative to S2 system motion.) Furthermore, as mentioned in section 3.3.2, the idea that parameterized convective momentum transport may be an important missing physical process is consistent with the finding that the system motion is likely largely advective, as lower-tropospheric advecting winds would be made stronger by downward momentum transport behind the convective line, increasing the system's eastward movement in an advective sense. However, while the UC in the S1 case study shown here likely moves largely by 
advection, it is important to note that not all $\mathrm{S} 1$ systems necessarily move by primarily advective means; indeed, several other S1 cases that were examined in real-time (not discussed here) do appear to possess larger propagative components in their storm motion.

The distinction between whether the system was being advected by the mean flow and whether it was propagating was hypothesized to be of great importance at the beginning of this study. However, the case studies have shown that the speed at which the UC moves relative to the main system is of even greater importance. This is a difficult evaluation to make in real-time (the question of what constitutes the "main system" can sometimes yield a nebulous answer), thus a differentiation based on katafront/anafront identification may instead be more appropriate. The UC in S1 cases appears to "outrun" the main system, often moving into the warm sector and interrupting flow from the south, whereas in S2 cases the UC generally stays collocated with the surface cold front (e.g., Fig. 5.1). Katafronts are generally considered to have precipitation in a band along or ahead of the cold front, while anafronts are characterized by postfrontal cloudiness and precipitation (e.g., Moore and Smith 1989). Therefore, katafronts may be helpful identifiers of S1 cases, and anafronts may indicate that an S2 event is in progress. Using the katafront vs. anafront idea to differentiate S1 cases from S2 cases is likely not foolproof ${ }^{1}$, but may offer a good starting point. This, and other forecast applications are further discussed in the following section.

\footnotetext{
${ }^{1}$ For example, some fronts exhibit katafront and anafront characteristics simultaneously along different sections of the front.
} 


\subsubsection{Forecasting considerations}

Over the course of this study, many events outside of the two major case studies were informally examined, with the goal of finding some delineating aspect of S1 or S2 that could easily illustrate to forecasters how to adjust downstream QPF in the presence of UC. Success in doing so was complicated by two major factors.

First, as discussed above, some UC events do not fit neatly into a particular "scenario"; each event is unique and no given event will perfectly match a simplistic schematic such as those outlined in Chapter 1. In much the same way, there is most likely no "magical" indicator that will always define what type of downstream QPF adjustment should be made; therefore, the identification and successful downstream forecasting of UC systems will likely require a more comprehensive examination of any given UC situation.

Second, the forecast problem in UC situations is two-fold. Not only is the downstream QPF often erroneous, but there is no guarantee that the model forecast of the UC feature will be accurate either. For example, a model forecast may represent the UC feature as a slow-moving anafront, but if the model forecast proves to be incorrect and observations reveal a fast-moving, bowing, katafrontal feature, then the adjustment that may have been made after looking at the initial model forecast of the UC would most likely be quite wrong. Therefore, there is often an important nowcasting component to the successful forecast of an UC situation.

However, despite these two major hurdles, the formal case studies, in addition to informal real-time event investigations, did shed some light on several characteristics that 
may be helpful in identifying and successfully forecasting many UC cases. These features are summarized in Table 5.1, and discussed in detail below.

S1 cases are often characterized by many of the same features that define an environment conducive to strong, organized multicellular convective systems. Moderate instability, significant deep-layer vertical wind shear, a strong upper-level trough, and strong westerly flow aloft are all common characteristics to the S1 environment. These signals were observed in several S1 cases that were examined in real-time over the 2004 - 2005 cold season, as well as in the S1 case study. As discussed before, if the system appears to be more of the katafront variety (i.e. the UC is located toward the front of or ahead of the frontal feature), then it is also likely more representative of an S1 environment. In a nowcasting sense, S1 cases can be identified by the speed at which the UC is moving (relative to the main system or surface front), as well as the alignment of the convection relative to the mean flow. A general bowing structure or bowing segments embedded within the line (evident in radar imagery) also offer compelling indications of a S1 case.

Conversely, S2 cases are often characterized by a lifting upper-level trough, and the UC may often align with the right entrance region of the jet stream associated with the confluent flow aloft. The magnitude of the deep-layer vertical wind shear tends to be weaker for S2 cases, and anafront characteristics (i.e. the UC is located just along or toward the rear of the surface front) may also indicate an S2 setup. Identifying a S2 case in a nowcasting sense is best done by monitoring the system speed (the UC should move slowly, especially relative to the surface front), and by tracking the speed of the LLJ and looking for any signs of intensification ahead of the UC. 
Finally, a useful NWP forecast parameter to evaluate for either scenario is the convective precipitation field (i.e. the precipitation produced by the $\mathrm{CP}$ scheme.) An important field to examine on a regular basis, the convective QPF is likely of particular importance for UC events. If a model forecast reveals that most of its QPF is produced by the CP scheme, then the uncertainty inherent to all CP schemes must be considered, and the forecast evaluated thusly (e.g. Mahoney and Lackmann 2005). As previously discussed, there are many limitations to the extent to which $\mathrm{CP}$ schemes can accurately simulate the effects of convection in a numerical model. CP schemes are limited in their physical accuracy, and it is recognized that the CP schemes may limit the physical accuracy of the representation of convection (e.g. Wang and Seaman 1997; Davis et al. 2003; Houze 2004). Of particular importance to this study of course is the challenge that quickly-moving convective systems pose to $\mathrm{CP}$ schemes, as well as the possible implications of the lack of representation of the momentum adjustment process. Therefore, the use of forecasts employing CP schemes must be evaluated with appropriate discrimination, particularly for cases featuring UC.

\subsection{Conclusions}

\subsubsection{Objectives}

This study examines the effect of UC on the downstream precipitation pattern, amounts, and forecasts. Prompted by the interest of NWS forecasters, this study investigates the various processes by which the presence of UC may impact the downstream QPF. Forecaster experience has shown that UC often degrades the downstream QPF, the resulting bias frequently being one of over-prediction in the 
downstream area. This investigation seeks to determine how the presence of UC physically alters the downstream environment and degrades the downstream precipitation forecast. The primary goals of this study are listed below, with the major finding concerning each goal listed in parentheses:

(i) identify the physical processes by which the downstream precipitation may be reduced or enhanced in the presence of UC (mechanism (iii) most important in case studies examined here);

(ii) distinguish UC cases in which downstream QPF should be reduced from those in which it should be enhanced (katafront vs. anafront a reliable signal);

(iii) understand why operational models are challenged to produce an accurate downstream forecast (errors in MCS motion, diabatic LLJ alteration);

(iv) identify synoptic settings associated with different types of UC events (S1, S2, S3 and associated characteristics from Table 5.1);

(v) find ways in which human forecasters may anticipate and improve upon erroneous model forecasts, (recognize scenario, then adjust model QPF), and;

(vi) investigate optimal model configurations for the representation of UC (highresolution, explicit convection).

\subsubsection{Hypotheses}

A brief climatology of Eta model QPF error allowed several UC cases to be selected and examined in an informal manner. Based on these preliminary investigations, 
as well as NWS forecaster input, the UC problem was divided into multiple scenarios, in which different QPF biases are hypothesized to occur. These three major scenarios are characterized by the orientation and movement of the UC, and each was hypothesized to have a different downstream QPF error implication.

$\mathrm{S} 1$ is characterized by $\mathrm{UC}$ that is oriented parallel to mean flow, and the convection propagates quickly relative to the primary system, in a direction perpendicular to flow (Fig. 1.6). Through possible mechanisms such as moisture removal/interception, stabilization of the downstream environment, or inaccurate MCS movement in the model forecast, these cases were observed to often show decreased downstream precipitation relative to the model QPF.

$\mathrm{S} 2$ features UC that is oriented parallel to mean flow as in $\mathrm{S} 1$, but that propagates slowly (or not at all) with respect to the primary system (Fig. 1.7). It is believed that a diabatically-enhanced low-level jet (LLJ) would act to enhance moisture transport ahead of such a system, and these cases were therefore hypothesized to yield increased downstream precipitation amounts, albeit with smaller errors (depending on model QPF accuracy earlier in event).

Finally, S3 is characterized by UC oriented perpendicular to flow, usually aligning with the coast and/or warm frontal features (Fig 1.8). S3 cases were hypothesized to show decreased downstream precipitation, possibly as a result of interrupted moisture transport.

In addition to these three scenarios, various physical processes were investigated for their potential role in downstream model QPF errors. The following mechanisms were hypothesized to play a possible role in the alteration of the downstream environment: 
(i) Moisture consumption (removal of moisture prior to its transport into the downstream area) (Fig. 1.2)

(ii) Stabilization of the downstream environment (elimination of instability in a way that reduces post-system convective precipitation, or leads to stable air being lifted synoptically with reduced ascent, as opposed to the lifting of unstable air) (Fig. 1.3)

(iii) Alteration of lower-tropospheric moisture transport through interruption (S1) or enhancement (S2) of the LLJ (Fig. 1.4)

(iv) Alteration of synoptic dynamics (e.g., downstream upper ridge building via latent heat release (LHR), reduction or elimination of synoptic forcing for forced ascent) (Fig. 1.5)

While the results of this study do not unambiguously identify which process is most important to a particular scenario, results from the case studies discussed in Chapters 3 and 4 do demonstrate significant differences in the physical alteration of the downstream environment for each scenario, discussed below.

\subsubsection{Case Studies and model experiments}

Two UC events were chosen to represent S1 and S2, respectively (a suitable S3 event could not be found using the QPF error climatology and was thus only informally investigated in the Appendix.) Each was examined from an observational and modeling perspective in order to gain insight on the specific processes that resulted in the downstream model QPF error for each respective scenario. 


\subsubsection{Scenario 1: 31 December 2002 - 1 January 2003}

On 31 Dec 2002, a squall line moving eastward along the coast of the Gulf of Mexico raced out ahead of its associated surface cold front. Operational QPF errors were in excess of $35 \mathrm{~mm}$ across western and central $\mathrm{SC} / \mathrm{NC} / \mathrm{VA}$, resulting in a major model QPF false alarm.

This event exemplifies S1, and it was therefore hypothesized that the fast-moving squall line featured in this case somehow impeded precipitation from falling where it was forecast to do so. Reasons for the large false alarm that resulted in the operational Eta model forecast were explored using numerical model experiments.

A 4-km explicit WRF model forecast was performed on this case, and it was found that both its representation of the UC, as well as its downstream QPF were superior to any of the model runs that had been performed using a CP scheme at a lower resolution. Examination of low-level flow and moisture flux fields suggests that the UC did act to suppress moisture transport to the north of the system, thus reducing downstream precipitation amounts. Therefore, only the model run performed without a $\mathrm{CP}$ scheme and at a high enough resolution to resolve convective motion was able to significantly improve the downstream QPF. This finding suggests that CP schemes may inherently preclude accurate forecasts of convective system motion and the QPF of their associated downstream environments in some S1 situations.

An Ertel PV budget was also performed for this case study, and its results showed that the movement of the convective system in this case was likely significantly advective in nature, with advective positive PV tendencies dominating along the leading edge of the system as the system evolved in time. 
Because the MCS moved too slowly in model forecasts that used a CP scheme, it is hypothesized that the omission of a physical process such as momentum adjustment in $\mathrm{CP}$ schemes may preclude accurate forecasts of convective system motion, as a large advective component of the flow is likely missing if this process is not accounted for. This problem is beyond the scope of this study, and is instead left for future investigation.

The S1 case study investigation supports the hypothesis that quickly-moving UC can act to decrease moisture to the downstream region, and thereby reduce downstream precipitation amounts relative to those forecasted by numerical models that use $\mathrm{CP}$ schemes.

\subsubsection{Scenario 2: $13-14$ January 2005}

On 13 - 14 Jan 2005 a slow-moving anafront produced large amounts of precipitation as it passed through the middle and eastern parts of the US. With respect to QPF, this case was an operational Eta model "miss" for parts of the Southeast and MidAtlantic regions of the US, as precipitation amounts were significantly under-forecasted at certain times and locations (a negative model QPF bias). As this case represented S2, it was hypothesized that the downstream precipitation amounts were underforecasted due to the inability of the operational models to properly account for the LHR that occurred in association with the heavy rainfall. Model inability to properly account for strong diabatic processes has been documented in the literature (e.g. Kuo et al. 1996; Dickinson et al. 1997; Lackmann et al. 2002), and it is believed that such processes were responsible for strengthening the pre-frontal LLJ, increasing moisture transport, and thereby increasing precipitation in the downstream area. 
PV diagnostics were used to demonstrate that the diabatic influence of the UC acted to enhance the LLJ and increase moisture transport. An Ertel PV budget showed that while large nonadvective PV tendencies were generated by the UC, the alignment and location of such tendencies did not act to move the system eastward, as seen in S1. A QGPV inversion showed that the diabatic cyclonic QGPV anomaly contributed significantly to the southerly LLJ preceding the convective line.

Therefore, S2 is an important counter-example to the notion that UC generally reduces downstream precipitation, as this case study demonstrates a physical process associated with the UC that actually acts to enhance downstream precipitation amounts.

\subsubsection{Forecasting implications}

The Eta QPF error climatology, in addition to informal, real-time case evaluations, has led to the hypothesis that S1 cases generally occur with more frequency and produce larger QPF errors than do S2 cases. This is not to diminish the significance of S2; rather, S2 confirms that UC is not always associated positive model QPF biases, and that there is a physical mechanism by which UC may also enhance downstream precipitation.

For forecast applications, it is important to develop the capability to distinguish between cases in which downstream precipitation may be reduced, and those in which it may be enhanced. These are summarized in Table 5.1, and may include checking for katafront or anafront characteristics, evaluating the upper air pattern, and carefully examining the speed and motion of the UC feature. It will require future collaboration over an extended period with those in a real-time forecast environment to further develop 
and fine-tune forecast tools with which to anticipate potential UC-event forecast adjustments.

This study also offers an important message to the NWP modeling community. At a time when NWP is moving toward ensemble forecast systems, decisions must be made regarding how to best use available computational resources (Roebber, et al. 2004). That is, are high-resolution, convection-permitting forecasts the best use of computer power, or is a large ensemble of lower-resolution forecasts optimal? The results found here suggest that for UC cases such as the S1 case study, only high-resolution explicit forecasts will be able to realistically resolve the physical processes necessary for accurate downstream QPFs. While ensembles of high-resolution, convection-resolving model forecasts is likely the ideal solution, computational limitations dictate that such decisions will have to be made, at least in the near future.

\subsubsection{Future work}

There are many avenues of future research that have been illuminated by this study. Given the S1 case study findings, the problem of the missing momentum adjustment process in $\mathrm{CP}$ schemes will be one area that will be investigated in ongoing research. It is hypothesized that the inclusion of this process may increase the possibility of an accurate forecast of a fast-moving MCS by permitting the contribution of the transported momentum to help propel the system forward. Such an improved forecast would further allow for an improved representation of the downstream environment.

There are many other questions that have been raised by the results of the two case studies that future research may also examine. With respect to $\mathrm{S} 1$, a higher- 
resolution run using a larger domain would likely greatly improve the representation of the UC system and eliminate the current problems that the limited domain size represents. Bryan et al. (2003) suggests using 1-km grid spacing or smaller; such simulations in the future are expected to improve even further upon the representation of the movement of the UC systems. To further improve upon the S1 investigation, performing a PV budget on a higher-resolution, more accurate model run would also be instructive (as excessive noise in such fields precluded that from being possible here.)

To further investigate S2, performing an Ertel PV inversion would eliminate the loss of accuracy that results from the use of QGPV, and would more accurately reveal the contribution of the diabatic PV feature on the LLJ and downstream moisture transport.

Finally, continued collaboration with operational forecasters in real-time forecast situations will be necessary in order to further improve forecasts of these events. With time, it is expected that useful tools and applications will be developed that will improve forecaster anticipation of erroneous operational model precipitation forecasts, as well as the ability to improve upon them. 


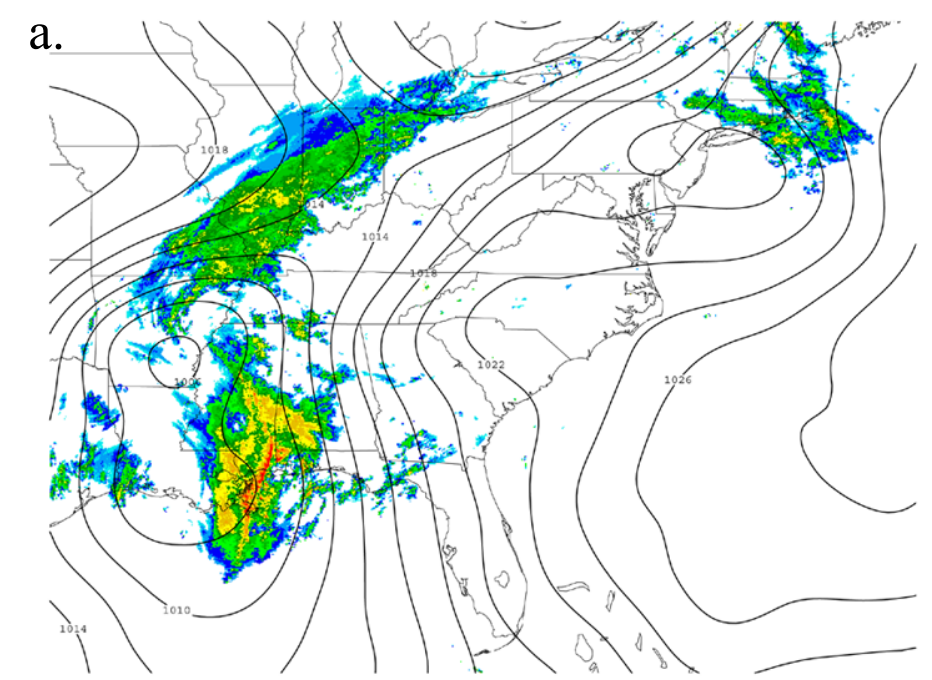

S1: 12 UTC 31 Dec 2002

$\underset{\infty}{\infty}$

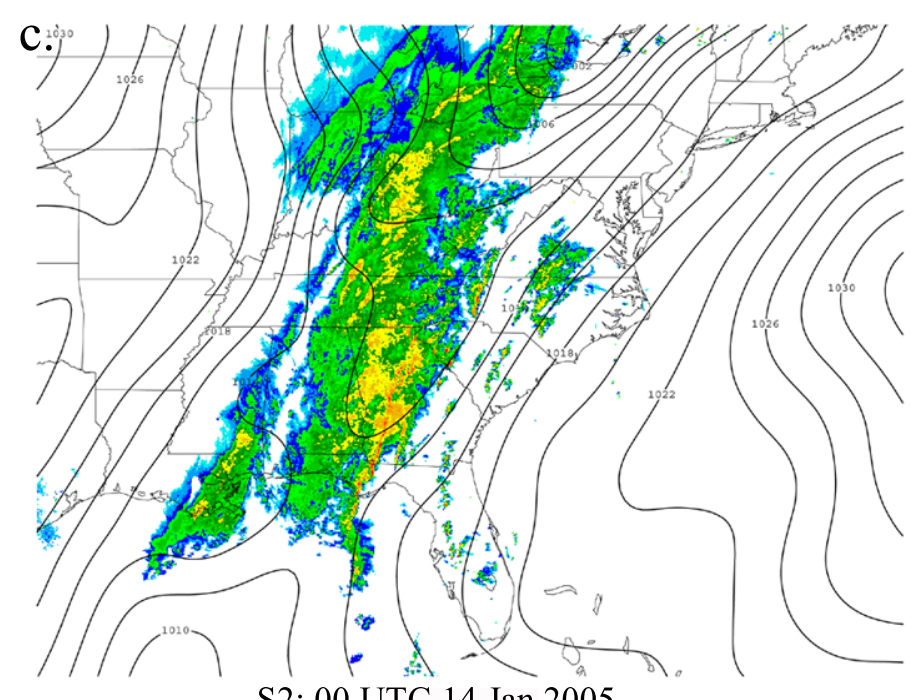

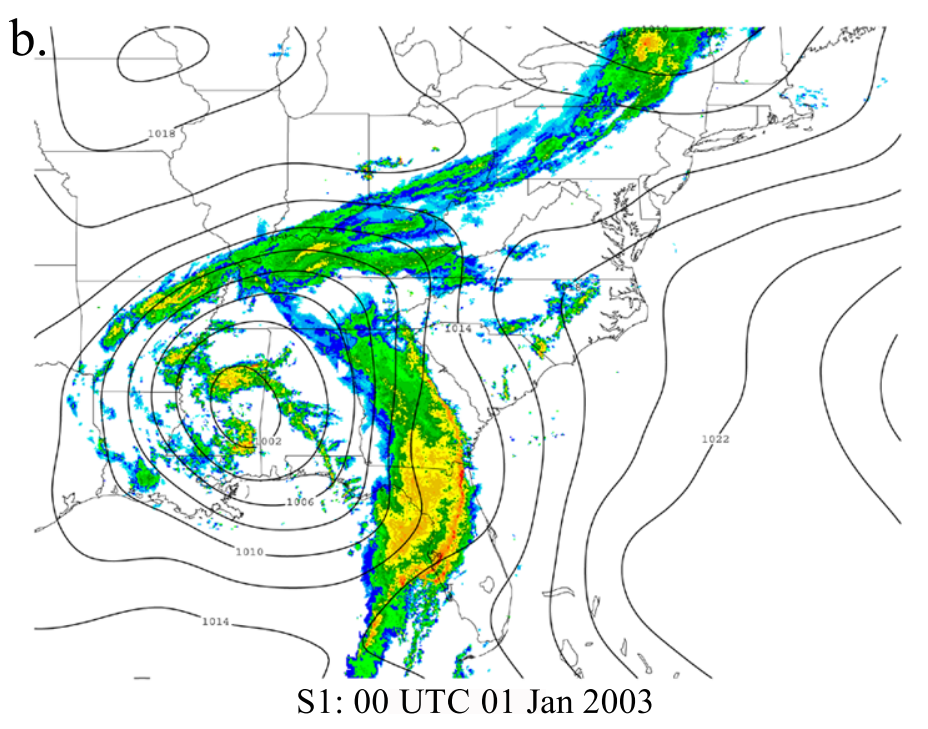

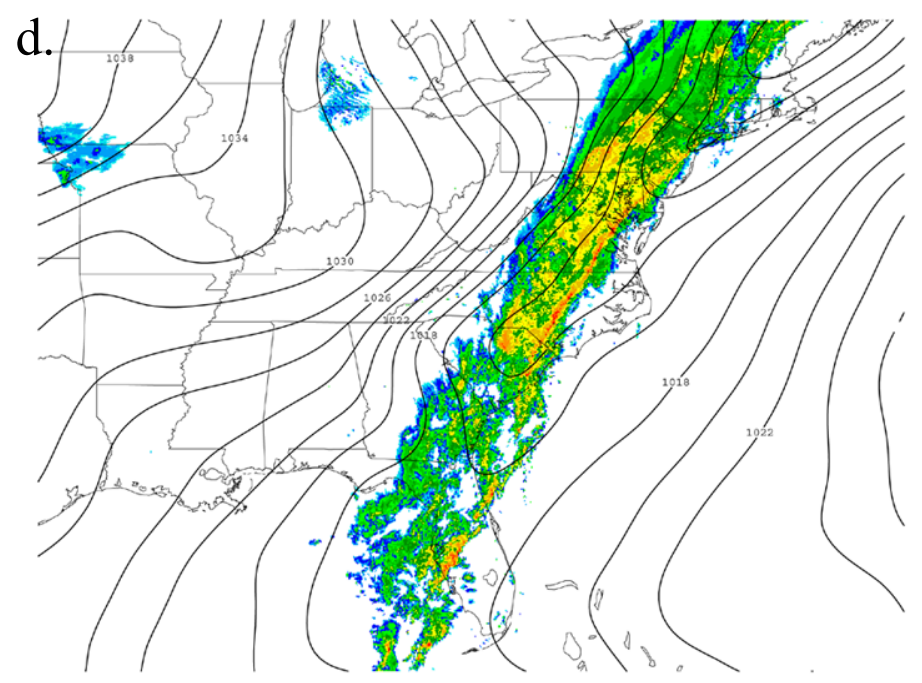

S2: 12 UTC 14 Jan 2005 2002; (b) as in (a)
Figure 5.1. (a) EDAS sea level pressure (solid, contour interval $2 \mathrm{hPa}$ ) and radar mosaic imagery valid 12 UIC 31 Dec 2002; 2005 except valid 00 UTC 01 Jan 2003; (c) as in (a) except valid 00 UTC 14 Jan 2005; (d) as in (a) except valid 12 UTC 14 Jan 2005. 


\begin{tabular}{|c|c|}
\hline Scenario 1 (S1) & Scenario 2 (S2) \\
\hline $\begin{array}{l}\text { Deep/digging upper trough (usually } \\
\text { located over lower Midwest) }\end{array}$ & $\begin{array}{l}\text { Confluent, lifting upper trough (favorable jet } \\
\text { dynamics in downstream region) }\end{array}$ \\
\hline Katafront characteristics & Anafront characteristics \\
\hline Significant shear/ strong westerlies aloft & Weaker shear, weaker flow aloft \\
\hline Bowing segments in UC & LLJ strength increasing in time \\
\hline
\end{tabular}

Table 5.1 Environmental characteristics common to S1 and S2 events 


\section{List of References}

Arakawa, A., 1993: Closure assumptions in the cumulus parameterization problem. The Representation of Cumulus Convection in Numerical Models, Meteor. Monogr., No. 46, Amer. Meteor. Soc., 1-15.

Bélair, S. and J. Mailhot, 2001: Impact of horizontal resolution on the numerical simulation of a midlatitude squall line: Implicit versus explicit condensation. Mon. Wea. Rev., 129, 2362-2376.

Bluestein, H. B., 1993: Synoptic-Dynamic Meteorology in Midlatitudes, Vol. II., Oxford University Press.

Braun, S. A., and R. A. Houze Jr., 1996: The heat budget of a midlatitude squall line and Implications for Potential Vorticity Production. J. Atmos. Sci., 53, 12171240.

- , and - 1997: The evolution of the 10-11 June 1985 PRE-STORM squall line: initiation, development of rear inflow, and dissipation. Mon. Wea. Rev., 125, 478504.

Brennan, M. J., and G. M. Lackmann, 2005: The influence of incipient latent heat release on the precipitation distribution of the 24-25 January 2000 cyclone. Mon. Wea. Rev., in press.

— , G.M. Lackmann, G.I. Hartfield, K.M. Mahoney, 2005: Operational use of PV thinking, in preparation.

Bryan, G. H., J. C. Wyngaard and J. M. Fritsch, 2003: Resolution requirements for the simulation of deep moist convection. Mon. Wea. Rev., 131, 2394-2416.

Cammas, J.-P., D. Keyser, G. M. Lackmann, and J. Molinari, 1994: Diabatic redistribution of potential vorticity accompanying the development of an outflow jet within a strong extratropical cyclone. Proc. Int. Symp. on the Life Cycles of Extratropical Cyclones, Vol. II, Bergen, Norway, Geophysical Institute, University of Bergen. 403-409.

Carbone, R. E., J. D. Tuttle, D. A. Ahijevych and S. B. Trier, 2002: Inferences of predictability associated with warm season precipitation episodes. J. Atmos. Sci., 59, 2033-2056.

Corfidi, S. F., 2003: Cold pools and MCS propagation: Forecasting the motion of downwind-developing MCSs. Wea. Forecasting, 18, 997-1017. 
_ J. H. Meritt and J. M. Fritsch, 1996: Predicting the movement of mesoscale convective complexes. Wea. Forecasting, 11, 41-46.

Crook, N. A., and M. W. Moncrieff, 1988: The effect of large-scale convergence on the generation and maintenance of deep moist convection. J. Atmos. Sci., 45, 36063624.

Davis, C. A., 1992a: A potential-vorticity diagnosis of the importance of initial structure and condensational heating in observed extratropical cyclogenesis. Mon. Wea. Rev., 120, 2409-2428.

—_ , 1992b: Piecewise potential vorticity inversion. J. Atmos. Sci., 49, 13971411.

_ , and K. A. Emanuel, 1991: Potential vorticity diagnostics of cyclogenesis. Mon. Wea. Rev., 119, 1929-1953.

__ , and M. L. Weisman., 1994: Balanced dynamics of mesoscale vortices produced in simulated convective systems. J. Atmos. Sci., 51, 2005-2030.

— , K. W. Manning, R. E. Carbone, S. B. Trier and J. D. Tuttle, 2003: Coherence of warm-season continental rainfall in numerical weather prediction models. Mon. Wea. Rev., 131, 2667-2679.

desJardins, M. L., K. F. Brill, and S. S. Schotz, 1991: GEMPAK 5 Part I-GEMPAK 5 programmer's guide. National Aeronautics and Space Administration. [Available from Scientific and Technical Information Division, Goddard Space Flight Center, Greenbelt, MD 20771.]

Dickinson, M. J., L. F. Bosart, W. E. Bracken, G. J. Hakim, D. M. Schultz, M. A. Bedrick and K. R. Tyle, 1997: The March 1993 Superstorm cyclogenesis: Incipient phase synoptic- and convective-scale flow interaction and model performance. Mon. Wea. Rev., 125, 3041-3072.

Done, J., C. Davis, and M. Weisman, 2003: The next generation of NWP: Explicit forecasts of convection using Weather Research and Forecasting (WRF) Model. Atmos. Sci. Lett. , DOI: 10.1002/asl.72.

Doswell, C.A., III, H. E. Brooks and R. A. Maddox, 1996: Flash flood forecasting: An ingredients-based methodology. Wea. Forecasting, 11, 560-581.

Dudhia, J., M. W. Moncrieff, and D. W. K. So, 1987: The two-dimensional dynamics of West Africa squall lines. Quart. J. Roy. Meteor. Soc., 113, 121-146.

Dudhia, J., 1993: A nonhydrostatic version of the Penn State-NCAR Mesoscale Model: 
Validation tests and simulation of an Atlantic cyclone and cold front. Mon. Wea. Rev., 121, 1493-1513.

Emanuel, K. A., 1986: Some dynamical aspects of precipitating convection. J. Atmos. Sci., 43, 2183-2198.

— M. Fantini and A. J. Thorpe, 1987: Baroclinic instability in an environment of small stability to slantwise moist convection. Part I: Two-dimensional models. $J$. Atmos. Sci., 44, 1559-1573.

_, D. Raymond, A. Betts, L. F. Bosart, C. Bretherton, K. Droegemeier, B. Farrell, J. M. Fritsch, R. Houze, M. LeMone, D. Lilly, R. Rotunno, M. Shapiro, R. Smith, and A. Thorpe, 1995: Report of the first prospectus development team of the U.S. Weather Research Program to NOAA and the NSF. Bull. Amer. Meteor. Soc., 76, 1194-1208.

Fortune, M., 1980: Properties of African squall lines inferred from time-lapse satellite imagery. Mon. Wea. Rev., 108, 153-168.

Fovell, R. G. and P.H. Tan, 1998: The temporal behavior of numerically simulated multicell-type storms. Part II: The convective cell life cycle and cell regeneration. Mon. Wea. Rev., 126, 551-577.

Fritsch, J. M., R. A. Houze, Jr., R. Adler, H. Bluestein, L. F. Bosart, J. Brown, F. Carr, C. Davis, R. H. Johnson, N. Junker, Y.-H. Kuo, S. Rutledge, J. Smith, Z. Toth, J. W. Wilson, E. Zipser, and D. Zrnic, 1998: Quantitative precipitation forecasting: Report of the eighth prospectus development team, U.S. Weather Research Program" Bull. Amer. Meteor. Soc., 79, 285-299.

— , and G.S. Forbes, 2001: Mesoscale convective systems. Severe Convective Storms, Meteor. Monogr., Amer. Meteor. Soc., 323-357.

— , and R. E. Carbone, 2004: Improving quantitative precipitation forecasts in the warm season: A USWRP research and development strategy. Bull. Amer. Meteor. Soc., 85, 955-965.

Gallus, W.A. Jr. and R. H. Johnson, 1992: The Momentum Budget of an Intense Midlatitude Squall Line. J. Atmos. Sci., 49, 422-450.

Gao, K., D-L Zhang, M. W. Moncrieff, and H-R Cho, 1990: Mesoscale Momentum Budget in a Midlatitude Squall Line: A Numerical Case Study. Mon. Wea. Rev., 118, 1011-1028.

Grell, G., Dudhia, J., and Stauffer, D., 1994: A description of the fifth generation Penn State/NCAR Mesoscale Model. NCAR Tech. Note NCAR/TN-398 + STR, 117 pp. 
Gyakum, J. R., 1983: On the evolution of the QE II Storm. II: Dynamic and thermodynamic structure. Mon. Wea. Rev., 111, 1156-1173.

Haertel, P. T. and R. H. Johnson, 2000: The linear dynamics of squall line mesohighs and wake lows. J. Atmos. Sci., 57, 93-107.

Hakim, G. J., D. Keyser and L. F. Bosart, 1996: The Ohio Valley wave-merger cyclogenesis event of 25-26 January 1978. Part II: Diagnosis using quasigeostrophic potential vorticity inversion. Mon. Wea. Rev., 124, 2176-2205.

Houze, R. A., Jr., 1977: Structure and dynamics of a tropical squall-line system. Mon. Wea. Rev., 105, 1540-1567.

— , 1993: Cloud dynamics. Academic Press, 570 pp.

—_ 2004: Mesoscale convective systems. Rev. Geophys., 42, 10.1029/2004RG000150, $43 \mathrm{pp}$.

— , and A. K. Betts, 1981: Convection in GATE. Rev. Geophys. Space Phys., 19, 541576.

Johns, R. H., 1984: A synoptic climatology of northwest-flow severe weather outbreaks. Part II: Meteorological parameters and synoptic patterns. Mon. Wea. Rev., 112, 449-464.

— , and W. D. Hirt, 1987: Derechos: Widespread convectively induced windstorms. Wea. Forecasting, 2, 32-49.

Kain, J. S., 2004: The Kain-Fritsch convective parameterization: An update. J. Appl. Meteor., 43, 170-181.

Keyser, D. and L. W. Uccellini, 1987: Regional models: Emerging research tools for synoptic meteorologists. Bull. Amer. Met. Soc., 68, 306-320.

Kuo, Y-H., R. J. Reed and Y. Liu, 1996: The ERICA IOP 5 Storm. Part III: Mesoscale cyclogenesis and precipitation parameterization. Mon. Wea. Rev., 124, 14091434.

Lackmann, G. M., 2002: Cold-frontal potential vorticity maxima, the low-level jet, and moisture transport in extratropical cyclones. Mon. Wea. Rev., 130, 59-74.

— , J. R. Gyakum and R. Benoit, 1998: Moisture transport diagnosis of a wintertime precipitation event in the Mackenzie River Basin. Mon. Wea. Rev., 126, 668-692. 
_ and J. R. Gyakum, 1999: Heavy cold-season precipitation in the Northwestern United States: Synoptic climatology and an analysis of the flood of 17-18 January 1986. Wea. Forecasting, 14, 687-700.

— , K. Keeter, L. G. Lee and M. B. Ek, 2002: Model representation of freezing and melting precipitation: Implications for winter weather forecasting. Wea. Forecasting, 17, 1016-1033.

Leary, C. A., and R. A. Houze Jr.,1979: Melting and evaporation of hydrometeors in precipitation from anvil clouds of deep tropical convection, J. Atmos. Sci., 36, 669-679.

Lin, Y-L., R. L. Deal and M. S. Kulie, 1998: Mechanisms of cell regeneration, development, and propagation within a two-dimensional multicell storm. $J$. Atmos. Sci., 55, 1867-1886.

_ , and Joyce, L. E., 2001: A further study of the mechanisms of cell regeneration, propagation, and development within two-dimensional multicell storms. J. Atmos. Sci., 58, 2957-2988.

Maddox, R. A., and C. A. Doswell, III, 1982: An examination of jet stream configurations, $500 \mathrm{mb}$ vorticity advection and low-level thermal advection patterns during extended periods of intense convection. Mon. Wea. Rev., 110, 184-197.

Mahoney, K.M. and Lackmann, G.M.: The Sensitivity of Numerical Forecasts to Convective Parameterization: A Case Study of the 17 February 2004 East Coast Cyclone. Wea. Forecasting, conditionally accepted.

Mesinger, F., and Coauthors: North American Regional Reanalysis. Bull. Amer. Met. Soc., submitted.

Merritt, J. H., and J. M. Fritsch, 1984: On movement of heavy precipitation areas of midlatitude mesoscale convective complexes. Preprints, $10^{\text {th }}$ Conf. On Weather Analysis and Forecasting, Clearwater, Amer. Meteor. Soc., 529-536.

Michalakes, J., S. Chen, J. Dudhia, L. Hart, J. Klemp, J. Middlecoff, and W. Skamarock (2001): Development of a Next Generation Regional Weather Research and Forecast Model. Developments in Teracomputing: Proceedings of the Ninth ECMWF Workshop on the Use of High Performance Computing in Meteorology. Eds. Walter Zwieflhofer and Norbert Kreitz. World Scientific, Singapore. pp. 269-276.

Molinari, J. and M. Dudek, 1992: Parameterization of convective precipitation in mesoscale numerical models: A critical review. Mon. Wea. Rev., 120, 326-344. 
Moore, J. T. and K. F. Smith, 1989: Diagnosis of anafronts and katafronts. Wea. Forecasting, 4, 61-72.

Newton, C. W., 1950: Structure and mechanism of the prefrontal squall line. J. Atmos. Sci., 7, 210-222.

Olson, D. A., N. W. Junker and B. Korty, 1995: Evaluation of 33 years of quantitative precipitation forecasting at the NMC. Wea. Forecasting, 10, 498-511.

Pandya, R. E. and D. R. Durran, 1996: The influence of convectively generated thermal forcing on the mesoscale circulation around squall lines. J. Atmos. Sci., 53, 29242951.

Raymond, D. J., 1992: Nonlinear balance and potential vorticity thinking at large Rossby number. Quart. J. Roy. Meteor. Soc., 118, 987-1015.

_ a and H. Jiang, 1990: A theory for long-lived mesoscale convective systems. J. Atmos. Sci., 47, 3067-3077.

Roebber, P. J., D. M. Schultz. B. A. Colle, and D. J. Stensrud, 2004: Toward improved prediction: High-resolution and ensemble modeling systems in operations. Wea. Forecasting: 19, 936-949.

Rogers, E., D. G. Deaven and G. S. Dimego, 1995: The regional analysis system for the operational "Early" Eta model: Original 80-km configuration and recent changes. Wea. Forecasting, 10, 810-825.

— , T. L. Black, D. G. Deaven, G. J. DiMego, Q. Zhao, M. Baldwin, N. W. Junker and Y. Lin. 1996: Changes to the operational "Early" Eta analysis/forecast system at the National Centers for Environmental Prediction. Wea. Forecasting, 11, 391-413.

Rotunno, R., J. B. Klemp, and M. L. Weisman, 1988: A theory for strong, long-lived squall lines. J. Atmos. Sci., 45, 463-485.

Schmidt, J. M. and W. R. Cotton, 1990: Interactions between upper and lower tropospheric gravity waves on squall line structure and maintenance. J. Atmos. Sci., 47, 1205-1222.

Skamarock, W. C., J. B. Klemp, J. Dudhia, D. O. Gill, D. M. Barker. W. Wang, and J. G. Powers, 2005: A description of the Advanced Research WRF Version 2. NCAR Tech. Note, NCAR/TN-468+STR, 100 pp.

Stoelinga, M. T., 1996: A potential vorticity-based study of the role of diabatic heating and friction in a numerically simulated baroclinic cyclone. Mon. Wea. Rev., 124, 849-874. 
— , J. D. Locatelli, R. D. Schwartz, P. V. Hobbs, 2003: Is a cold pool necessary for the maintenance of a squall line produced by a cold front aloft?. Mon. Wea. Rev., 131, $95-115$.

Thorpe, A. J., M. J. Miller and M. W. Moncrieff, 1982: Two-dimensional convection in non-constant shear: a model of mid-latitude squall lines. Quart. J. Roy. Meteor. Soc., 108, 739-762.

Waldstreicher, J., and D. Figurskey, 2005, personal communication, CSTAR List emails.

Wang, W., Y-H. Kuo and T. T. Warner, 1993: A diabatically driven mesoscale vortex in the lee of the Tibetan Plateau. Mon. Wea. Rev., 121, 2542-2561.

— , and N. L. Seaman, 1997: A comparison study of convective parameterization schemes in a mesoscale model. Mon. Wea. Rev., 125, 252-278.

Weisman, M.L., and J.B. Klemp, 1982: The dependence of numerically simulated convective storms on vertical wind shear and buoyancy. Mon. Wea. Rev., 110, $504-520$.

— , and J. B. Klemp, 1986: Characteristics of isolated convective storms (Chap. 15). Mesoscale Meteorology and Forecasting (P. Ray, ed.), Amer. Meteor. Soc., Boston, 331-358.

— , W. C. Skamarock and J. B. Klemp, 1997: The resolution dependence of explicitly modeled convective systems. Mon. Wea. Rev., 125, 527-548.

Whitaker, J. S., L. W. Uccellini, and K. F. Brill, 1988: A model-based diagnostic study of the rapid development phase of the Presidents's Day Cyclone. Mon. Wea. Rev., 116, 2337-2365.

Yang, M-J., and R. A. Houze, Jr., 1995: Multicell squall-line structure as a manifestation of vertically trapped gravity waves. Mon. Wea. Rev., 123, 641-661.

Zapotocny, T. H. , S. J. Nieman, W. P. Menzel, J. P. Nelson III, J. A. Jung, E. Rogers, D. F. Parrish, G. J. DiMego, M. Baldwin and T. J. Schmit. 2000: A case study of the sensitivity of the Eta Data Assimilation System. Wea. Forecasting, 15, 603-621.

Zhang, D-L., and K. Gao, 1989: Numerical simulation of an intense squall line during 10-11 June 1985 PRE-STORM. Part II: Rear inflow, surface pressure perturbations and stratiform precipitation. Mon. Wea. Rev., 117, 2067-2094.

Zhang, F., C. Snyder, and R. Rotunno, 2002: Mesoscale predictability of the "surprise" snowstorm of 24-25 January 2000. Mon. Wea. Rev., 130, 1617-1632. 


\section{Appendix: Scenario 3 examples}

Several examples of events that initially resembled the S3 schematic were examined. One such event occurred on 27 - 28 March 2005. Figure A1 shows the evolution of the S3 coast-parallel warm-frontal convection as it transitions into a strong pre-cold-frontal squall line, eventually reflecting more of a S1 setup. The east-westoriented UC (Fig. A1a) takes on more of a north-south orientation as the surface cold front further develops to the south of the original warm front (Fig. A1c), becoming more parallel than perpendicular to the low-level flow in time. Figure A2 shows the operational Eta 30-h QPF for this event and Fig. A3 shows the NPVU precipitation analysis. Comparing these two figures shows an Eta model QPF false alarm over NC and VA of more than half of an inch in some areas. Therefore, this S3/S1 hybrid event begins with a strong S3 signature and ends with a strong S1 signature, revealing limited downstream precipitation as was suggested in the original $\mathrm{S} 3$ hypothesis.

Another S3 example occurred on 12 - 13 March 2001. Again, the system initially looked like a S3 event, starting out with coast-parallel warm-frontal convection along the northern Gulf coast, but evolving in time into a more N-S oriented squall line, moving quickly eastward. Figure A4 shows the evolution of this event using radar imagery, from which the transition from $\mathrm{S} 3$ to $\mathrm{S} 1$ is evident. Also similar to the above case, a QPF false alarm in the downstream area over NC and VA occurred, as shown in Figs. A5 and A6.

Given what little that has been observed about S3 cases (or cases that initially resemble S3), these events appear to pose a different sort of a problem, with a separate host of challenges and potential research questions. Some S3 cases may evolve into something more closely resembling S1 or S2, while others may remain S3 but may not 
necessarily pose a significant threat to the downstream model QPF. While the QPF bias for cases resembling S3 may often be similar to that of an S1 case, the mechanism by which the over-prediction occurs is likely quite different. While S1 and S2 almost always feature UC that is either aligned with a cold front or located in the warm sector of a surface cyclone, S3 cases seem to be primarily warm-frontal "precursor" types of events. Furthermore, in most S1 and S2 events, the UC is largely surface-based, whereas convection north of the warm front (as in S3) is largely of the elevated variety.

In $\mathrm{S} 1$, the nature of the problem is a model inability to propagate convection quickly enough for some fast-moving systems. For S3, it is likely more of a model inability to represent elevated convection and the flow around such a feature. For warm frontal convection, the models are challenged to not only represent the initial moisture field accurately, but to also represent the process of layer-lifting, accurately transition from the $\mathrm{CP}$ scheme to the explicit precipitation physics, as well as be able resolve elevated convection. Such challenges are difficult to overcome, particularly using current operational NWP models, and therefore closer investigation of this S3 problem is left for future study. 

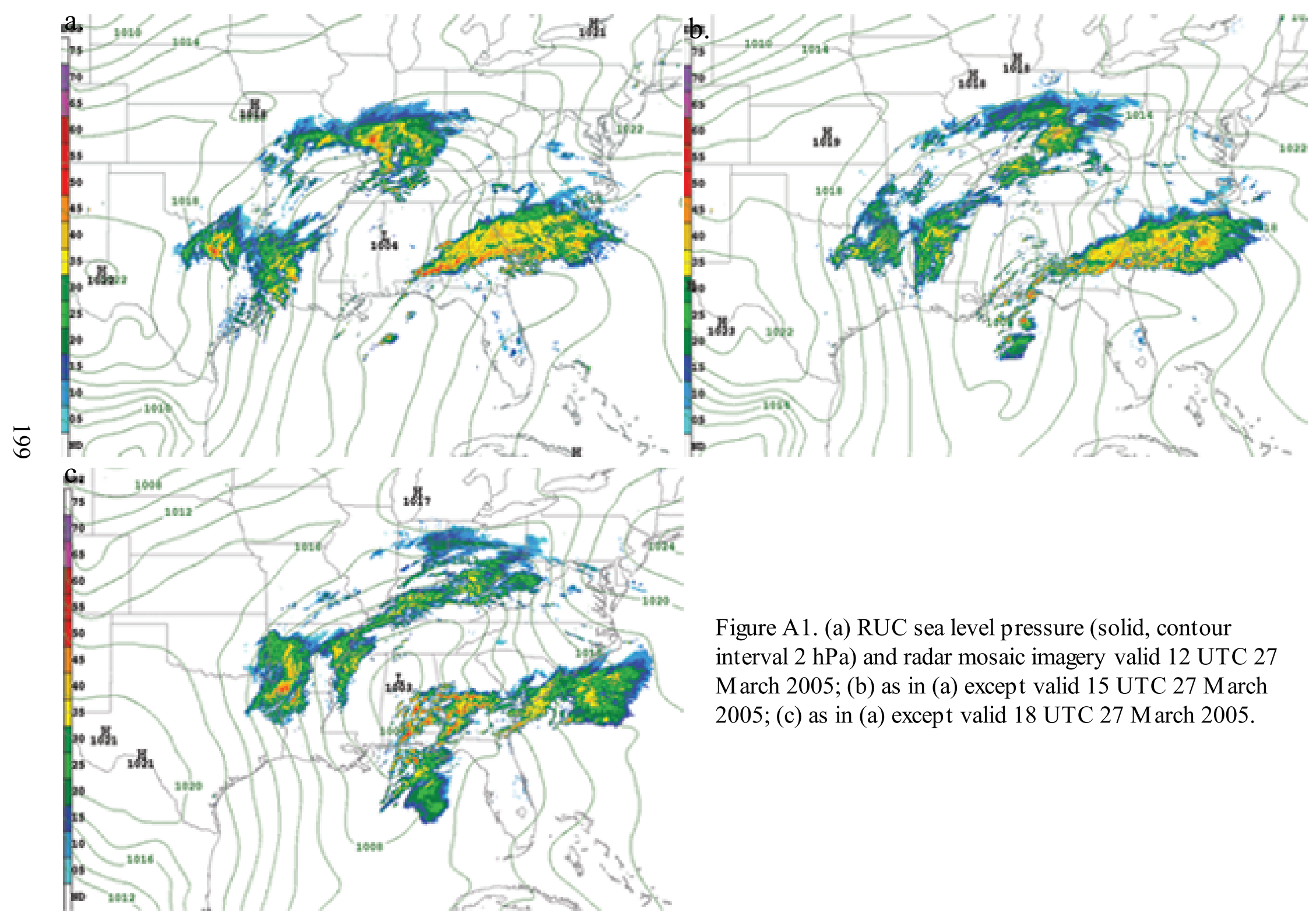

Figure A1. (a) RUC sea level pressure (solid, contour interval $2 \mathrm{hPa}$ ) and radar mosaic imagery valid 12 UTC 27 March 2005; (b) as in (a) except valid 15 UTC $27 \mathrm{March}$ 2005; (c) as in (a) except valid 18 UTC 27 March 2005. 


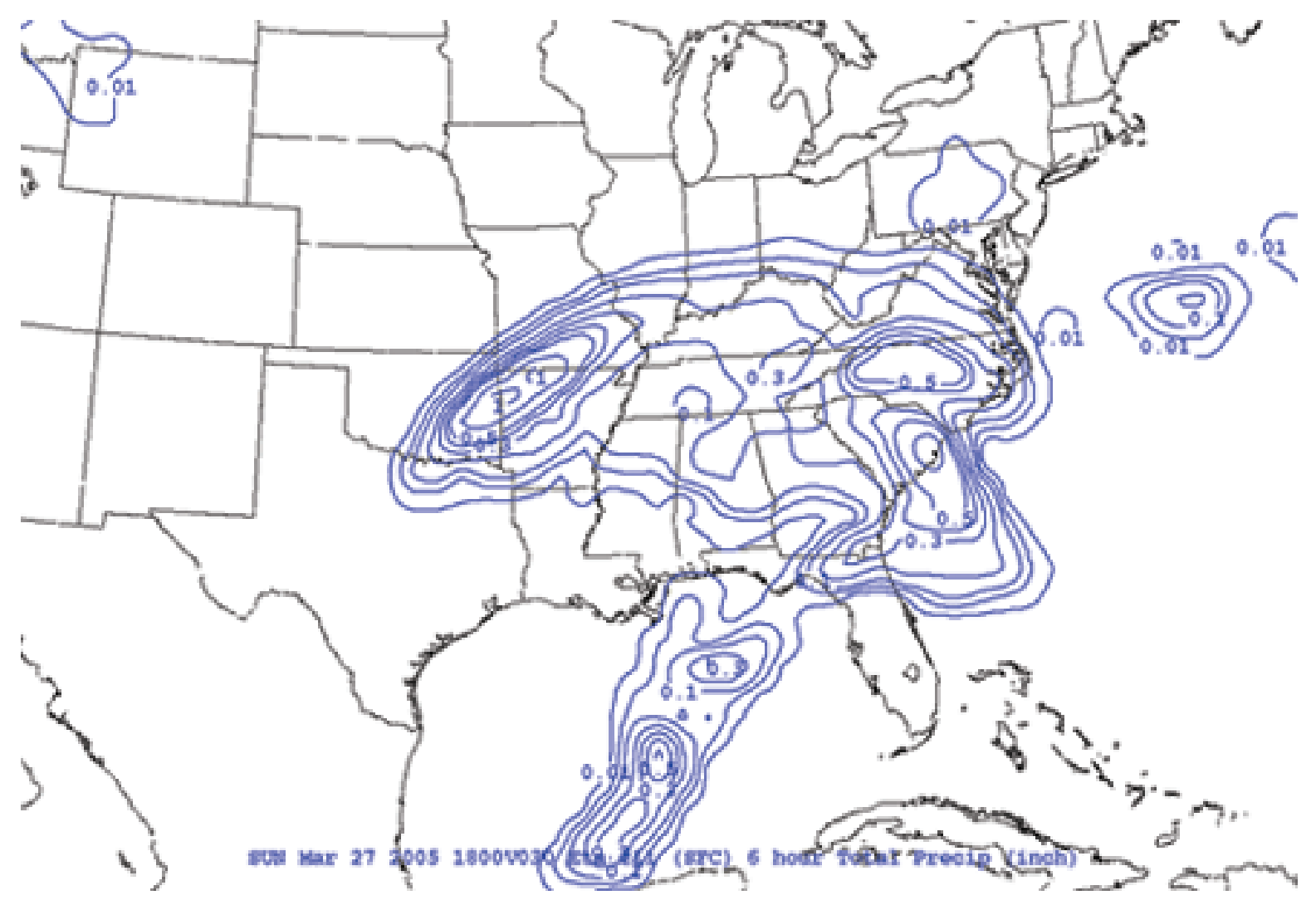

Figure A2. Operational Eta model 6-h total precipitation forecast ending 18 UTC 27 March 2005 (solid contours). 


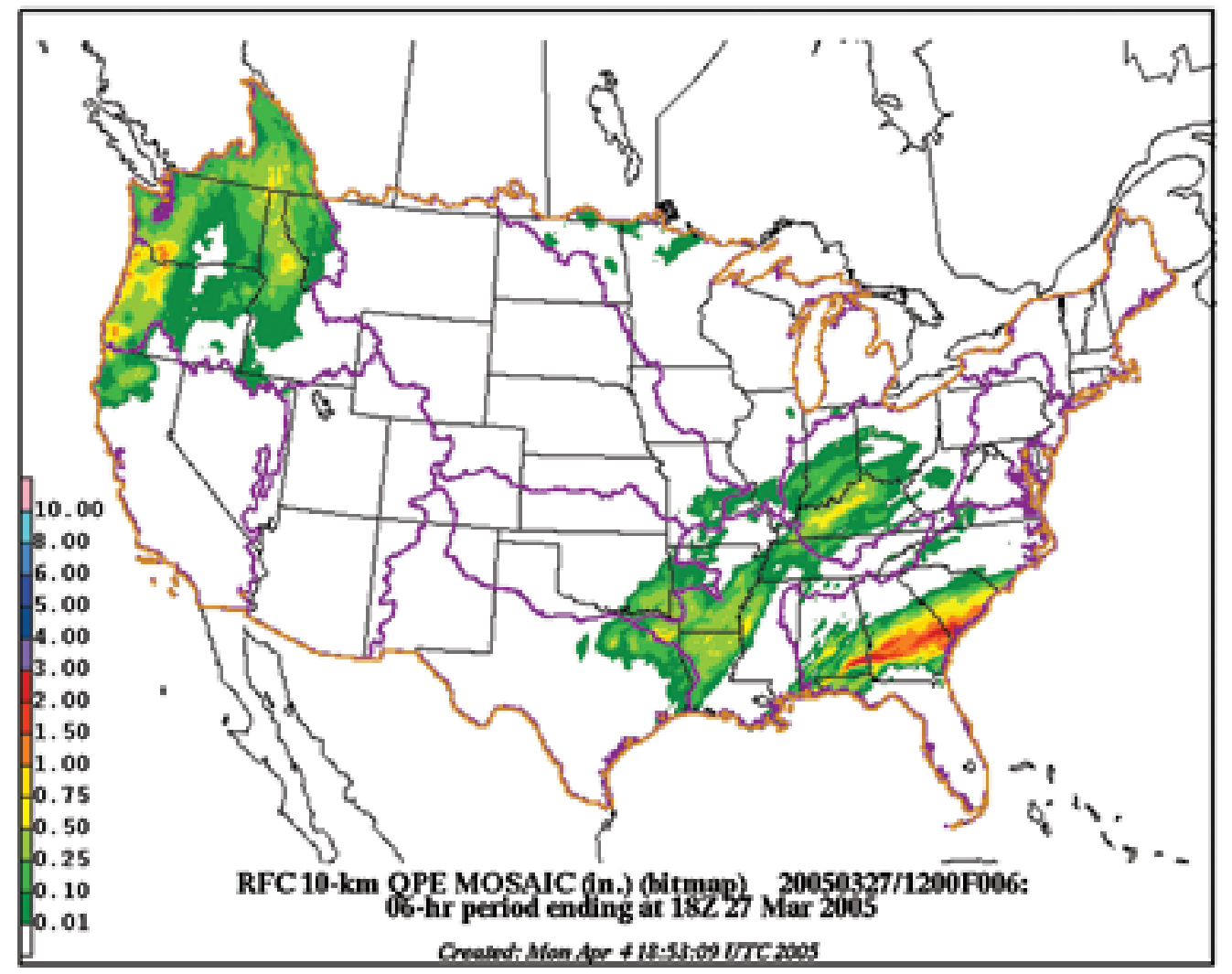

Figure A3. 6-h precipitation estimate from the National Precipitation Verification Unit (NPVU) online archive valid for 6-h period ending 18 UTC $27 \mathrm{March} 2005$ (in, shaded as in legend at left of panel). 


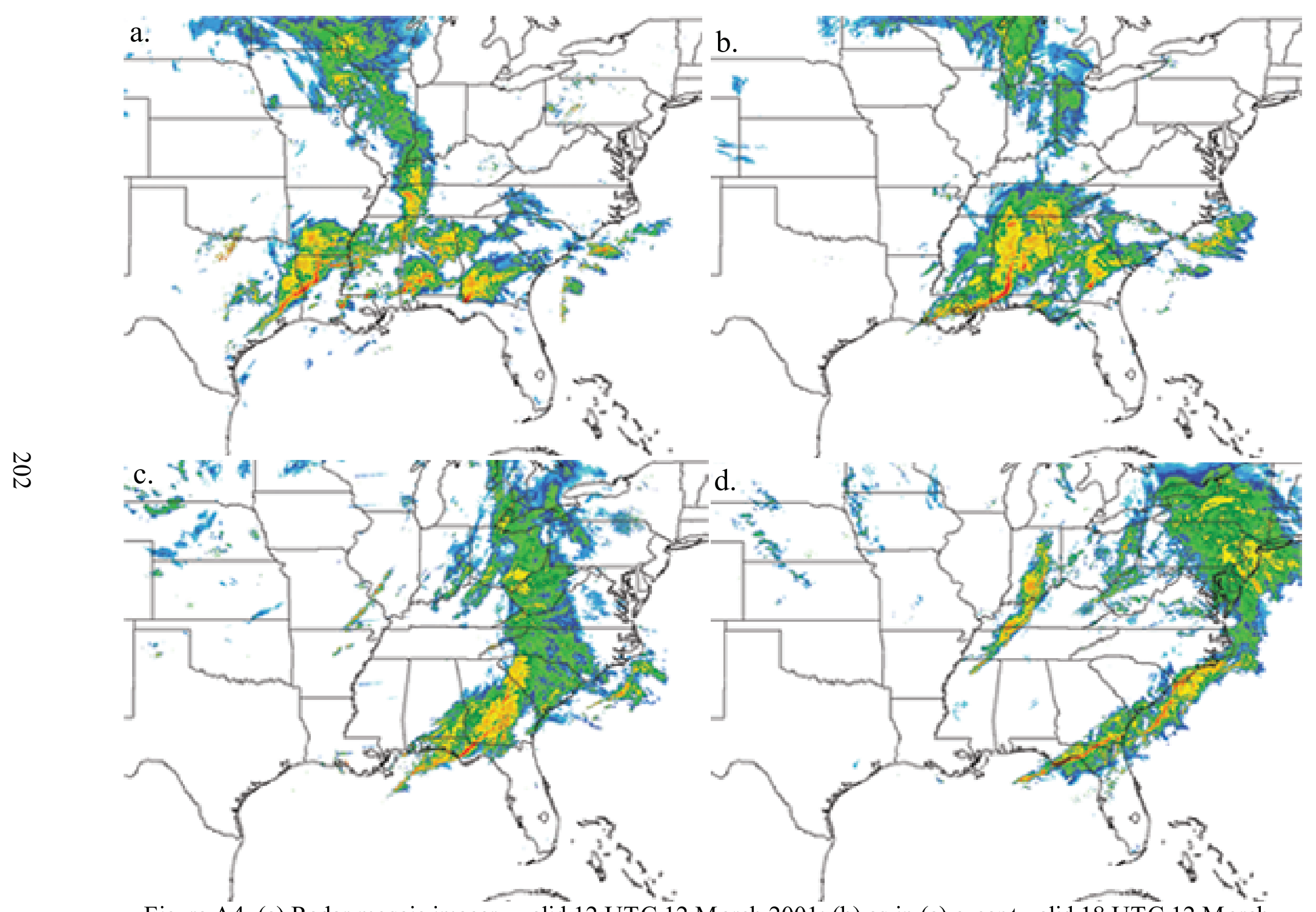

Figure A4. (a) Radar mosaic imagery valid 12 UTC 12 March 2001; (b) as in (a) except valid 18 UTC 12 March 2001; (c) as in (b) except valid 00 UTC 13 March 2001; (d) as in (a) except valid 06 UTC 13 March 2001. 


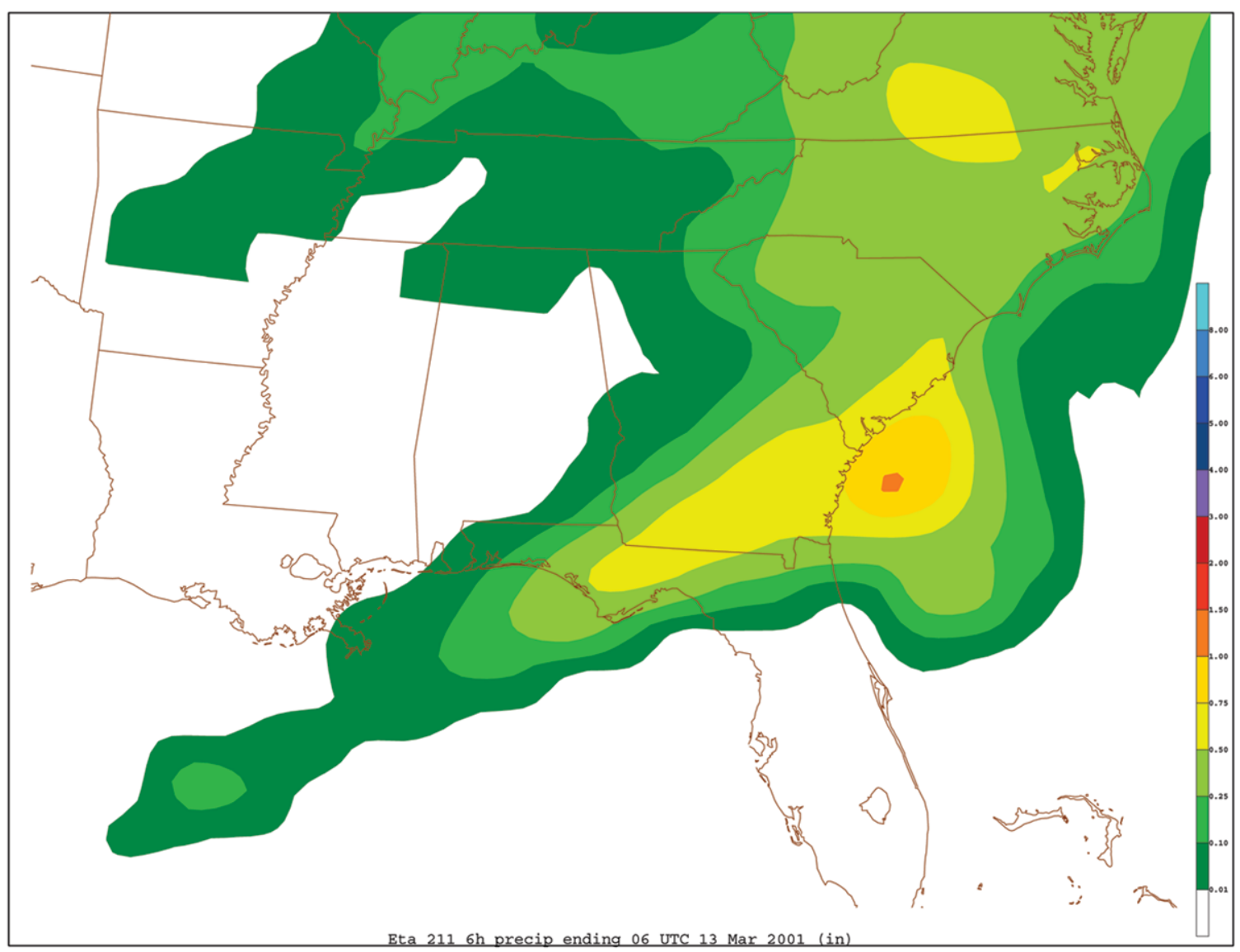

Figure A5. Operational Eta model 6-h total precipitation forecast ending 06 UTC 13 March 2001 (in, shaded as in legend at right of panel). 


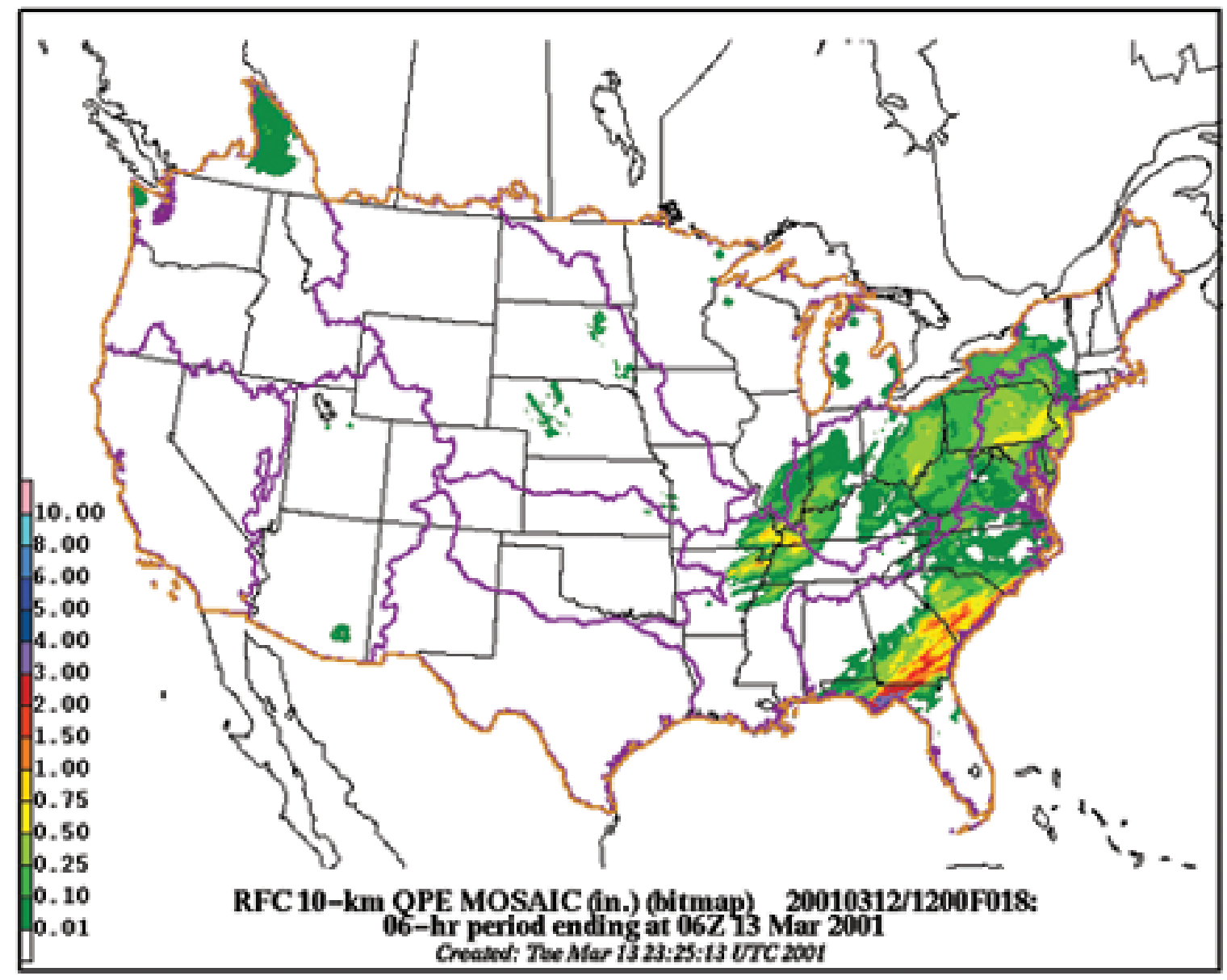

Figure A6. 6-h precip itation estimate from the National Precipitation Verification Unit (NPVU) online archive valid for 6-h period ending 06 UTC $13 \mathrm{March} 2001$ (in, shaded as in legend at left of panel). 